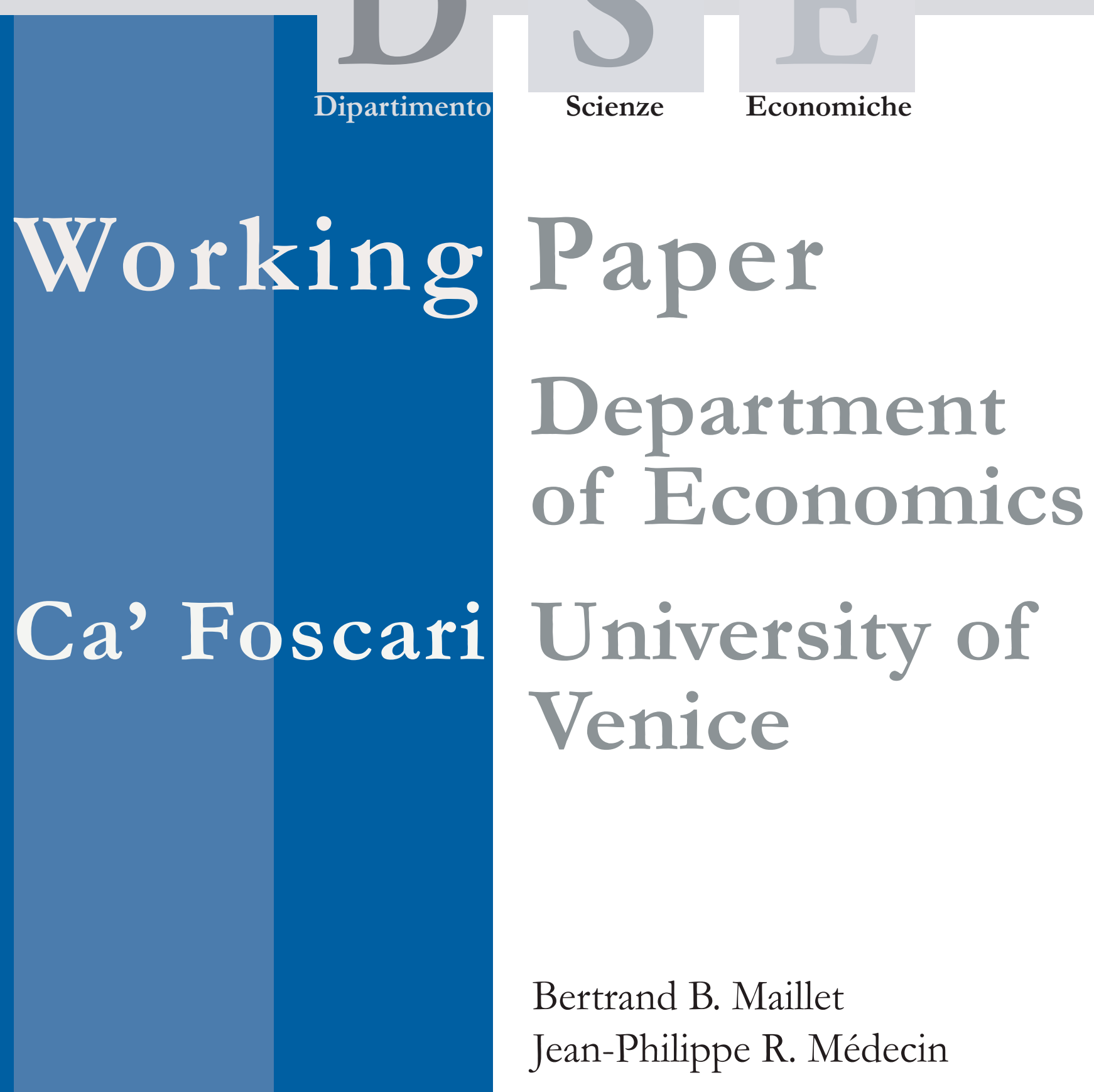

Extreme Volatilities, Financial

Crises and L-moment Estimations of Tail-indexes 


\title{
Extreme Volatilities, Financial Crises and L-moment Estimations of Tail-indexes*
}

\author{
Bertrand B. Maillet \\ ABN AMRO Advisors, Variances \\ and University of Paris-1 (CES/CNRS and EIF) \\ Jean-Philippe R. Médecin \\ Paris School of Economics, University of Paris-1 \\ and Variances
}

First Draft: April 2010

\begin{abstract}
Following Bali and Weinbaum (2005) and Maillet et al. (2010), we present several estimates of volatilities computed with high- and low-frequency data and complement their results using additional measures of risk and several alternative methods for Tail-index estimation. The aim here is to confirm previous results regarding the slope of the tail of various risk measure distributions, in order to define the high watermarks of market risks. We also produce synthetic general results concerning the method of estimation of the Tail-indexes related to expressions of the L-moments. Based on estimates of Tail-indexes, retrieved from the high frequency 30' sampled CAC40 French stock Index series from the period 1997-2009, using Non-parametric Generalized Hill, Maximum Likelihood and various kinds of L-moment Methods for the estimation of both a Generalized Extreme Value density and a Generalized Pareto Distribution, we confirm that a heavy-tail density specification of the Log-volatility is not necessary.

Keywords

Financial Crisis, Realized Volatility, Range-based Volatility, Extreme Value Distributions, Tailindex, L-moments, High Frequency Data.

\section{JEL Codes}

G.10, G.14.

Address for correspondence:

Bertrand B. Maillet

Visiting Academic at the Department of Economics

Ca' Foscari University of Venice Cannaregio 873, Fondamenta S.Giobbe 30121 Venezia - Italy

e-mail: bmaillet@univ-parsi1.fr
\end{abstract}

This Working Paper is published under the auspices of the Department of Economics of the Ca' Foscari University of Venice. Opinions expressed herein are those of the authors and not those of the Department. The Working Paper series is designed to divulge preliminary or incomplete work, circulated to favour discussion and comments. Citation of this paper should consider its provisional character.

* We are grateful to Charles Corrado for encouragement in preparing this work, as well as Patrick Kouontchou, Gregrory Jannin and Tristan Roger for research assistance. We also thank Tim Bollerslev, Christian Bontemps, Thierry Chauveau, Thierry Foucault, Sylvain Friederich, Jonathan Hosking, Alan Hutson, Olivier Le Courtois, Yannick Malevergne, Nour Meddahi, Roel Oomen, Richard Payne, Loriana Pelizzon, Didier Rullière, Konstantinos Tolikas and Dick van Dijk for useful comments. We finally aknowledge Thierry Michel for an intensive collaboration on earlier research on this subject. The first author thanks the Europlace Institute of Finance for financial support. This draft was finalized when he was a Visiting Professor at the Departimento di Scienze Economiche di San Giobbe dell' Università Ca' Foscari di Venezia. The usual disclaimers apply.

The Working Paper Series is available only on line (www.dse.unive.it/pubblicazioni) For editorial correspondence, please contact: wp.dse@unive.it
Department of Economics

Ca' Foscari University of Venice

Cannaregio 873, Fondamenta San Giobbe

30121 Venice Italy

Fax: ++390412349210 


"Extreme Volatilities, Financial Crises
and L-moment Estimations of Tail-indexes"

\section{Introduction}

The volatility has long been used as an auxiliary variable in the processes explaining the returns on risky assets. In this traditional framework, the observable were the returns and the volatility remained a latent variable, whose value was a by-product of the estimation. Recently, the focus has changed and many studies have been devoted to empirical estimates of the volatility itself, without necessarily specifying any model for the prices themselves. The measure of risk most widely used by practitioners remains today the Close-to-Close Simple Volatility, a time-varying simile of the traditional variance.

Following for instance Barndorff-Nielsen and Shephard (2003) or Andersen et al. (2003), volatility can indeed be viewed as a latent factor (the so-called quadratic variation affecting the Brownian motion in some representations) that can only be estimated using its signature on market prices. It is only when the process is known (or simulated) as in Andersen and Bollerslev (1998-a) or Barndorff-Nielsen and Shephard (2002-a) that we know what the "true volatility" is (inside the Brownian motion case - see Andersen et al., 2003, for a survey). When the underlying process is more sophisticated, as shown by BarndorffNielsen and Shephard (2002-b), or when observed prices suffer from market microstructure distortion effects or high-frequency seasonalities, the results are less clear (see Andersen and Bollerslev, 1997-a, 1997-b and 1998-b ; Corsi et al., 2001; Bai et al., 2001; Martens, 2001; Martens et al., 2002; Oomen, 2004; Brandt and Diebold, 2006; Bandi and Russell, 2006).

On the one hand, the Realized Volatility is considered, since its introduction (see Andersen and Bollerslev, 1998-a), as the best estimator for the latent factor. The daily volatility retrieved from transaction data has been shown to be accurate when controlling the microstructure effects and empirically supporting the Clark (1973) Mixture of Distribution Hypothesis (Andersen et al., 2003). Among the high-frequency estimators, the one using all the available transactions (Cf. Bollen and Inder, 2002) is shown to perform better than the Realized Volatilities that use a lower sampling rate. Oomen (2005) also shows that estimating the volatility in business-time (transaction-time) is more efficient than using the traditional calendar-time, as it samples the process when it the most informative. Some authors recently show that the most accurate estimator, so far, is the mean of the Realized Volatilities chosen at the optimal frequency but measured at different phases (see Zhang et al., 2005; Aït-Sahalia et al., 2005 and 2009; Zhang, 2006).

On the other hand, when high-frequency data is unavailable - which is still the case in the majority of published studies in finance, second best estimations of the unobservable risk factor are provided by the Range-based - or Extreme Value - estimators as shown by an extensive comparative study by Bali and Weinbaum (2005). The price range, defined as the difference between the highest 
and lowest market prices over a fixed sampling interval, and often represented in business newspapers and available on financial data providers under the label "(Japanese) candlestick plots", is known for a long time as a volatility estimator. Back to Parkinson (1980), there is a wealth of literature ${ }^{1}$ devoted to refinements of this measure - using various assumptions on the underlying process.

Following Bali and Weinbaum (2005) and Maillet et al. (2010), the aim of the present article is to, furthermore, study the main high and low-frequency measures of volatility - focusing on extreme events, in order to find if there are glaring discrepancies between the empirical evidence and the usual assumptions about the distribution of volatility. The distribution will be estimated using various techniques including different L-moment methods.

Introduced by Sillitto (1951) and comprehensively reviewed and popularized by Hosking $(1986,1990)$, L-moments are demonstrably superior to classical algebraic moments since they are less subject to outliers and then to bias in estimation in small samples (e.g. Hosking, 1992; Ulrich et al., 2000; Delicado and Goria, 2008). L-moment expressions are based on linear combinations of order statistics and can be also seen as a modification of the Probability-weighted Moments (Greenwood et al., 1979). The estimation method relying on L-moments is now widely used for estimation of frequency of extreme events, which can be found in meteorology or hydrology fields, or more generally in regional analysis ( Cf. Hosking and Wallis, 1997). More precisely, it has been applied to studying the extreme distribution of floods (see Hosking and Wallis, 1987; Ben-Zvi and Azmon, 1997; Wang, 1997; Bayazit and Önöz, 2002; Moisello, 2007; Hewa et al., 2007; Shao et al., 2008), of rainfalls (e.g. Guttman et al., 1993; Lee and Maeng, 2003; De Michele and Salvadori, 2003; Parida and Moalafhi, 2008) of raindrop sizes ( $C f$. Kliche et al., 2008), of wind speed (e.g. Pandey et al., 2001; Whalen et al., 2004; Modarres, 2008) or to the measurement of earthquake intensities (Thompson et al., 2007). They have also found applications in finance for fitting return distributions (Hosking et al., 2000; Carillo et al., 2006-a; Karvanen, 2006) and rate of profit densities (Wells, 2007), for calibrating extreme returns (Gettinby et al., 2006; French, 2008; Tolikas and Gettinby, 2009), to elaborate a GMM-type Goodness-of-Fit test ( $C f$. Chu and Salmon, 2008), for risk modelling purposes ( $C f$. da Silva and de Melo Mendes, 2003; Tolikas and Brown, 2006; Martins-Filho and Yao, 2006; Tolikas et al., 2007; Tolikas, 2008; Gouriéroux and Jasiak, 2008; French, 2008), and, finally, for defining a new set of measures of performance for hedge funds ( $C f$. Darolles et al., 2009).

In this work, we first aim to compare the behavior of extreme volatilities, measured by several proxies that include the popular Realized Volatility (Andersen and Bollerslev, 1998-a). Our work secondly relies on the Extreme Value Theory. Its applications are now widely disseminated among the finance community (see the reference books by Embrecht et al. 1997; Adler et al., 1998; Coles, 2001; Finkenstädt and Rootzén, 2003; Beirlant et al., 2004; McNeil et al.,

\footnotetext{
${ }^{1}$ Relevant literature includes Parkinson (1980), Garman and Klass (1980), Rogers and Satchell (1991), Kunitomo (1992) and Yang and Zhang (2000). For the sake of completeness, we should also mention works by Ball and Torous (1983), Brunetti and Lildholdt (2002), Bollen and Inder (2002, 2003), and Høg and Lunde (2003).
} 
2005; Malevergne and Sornette, 2006; de Haan and Ferreira, 2006), specifically when studying the density of returns (see Longin, 1996) and potential severe losses (Bali, 2003; Tolikas, 2008). Based on works by Hosking (1990), we thirdly present several methods for estimating the Tail-index of the extreme volatility density, focusing more intensively on various variants of L-moments, that finally give us some confidence when gauging the high watermarks of market risks. The closest contributions to our paper have been published in fields such as Econometrics (Andersen et al., 2003; Bontemps and Meddahi, 2005; Christensen and Podolskij, 2007; Martens and van Dijk, 2007; Saichev et al., 2010), Financial Econometrics (Barndorff-Nielsen and Shephard, 2004; Gonzalo and Olmo, 2004; Oomen, 2005; Browlees and Gallo, 2010), Empirical Finance (Danielsson and de Vries, 1997; Bollen and Inder, 2002; Jondeau and Rockinger, 2003; Jalal and Rockinger, 2008; Tolikas and Gettinby, 2009), and Extremes Studies (Hosking et al., 1985; Beirlant et al., 1996; Resnick, 1997).

The paper is organized as follows. First, we recall the expression of main measures of volatility using transaction high-frequency data and Close-to-Close / Range low-frequency returns. Secondly, we present the main non-parametric and parametric (Generalized Extreme Value and Generalized Pareto Distributions) methods linked to studies of extreme volatilities, with a special focus on the methods related to L-moments. Thirdly, we conduct an empirical study of the main volatility estimators on a sample of high frequency quotes of the CAC40 French stock market Index from 1997 to 2009. The last section of the paper concludes, whilst the Appendices are reserved for the expressions of Extreme Value densities as a function of the various variants of L-moments (and for figures and tables).

\section{From High to Low Frequency Measures of Risk via Extreme Value Estimators of Volatility}

It has been known since the first attempts at modelling prices that the amplitude of price changes was not constant, but fluctuating with time in a somewhat predictable fashion. Though it is not possible to estimate the spot volatility (i.e. the value of the volatility at a point in time), the integrated variance (i.e. the variance of the instantaneous returns over a period) can be approximated through estimators of the quadratic variation (the limit of the sum of squared returns over decreasing time intervals). We present hereafter the main measures of daily volatility, both from intra-day data (that should converge to quadratic variation in the absence of jumps as shown by Barndorff-Nielsen and Shephard, 2003) and daily data (the range-based estimators being less sensitive to outliers and jumps than the squared returns). Following Andersen et al. (2009-a), we also present the Realized Volatility decomposition in a Jump Component and a remaining Bi-power Variation.

A simple direct extension of the traditional continuous time model for stock returns assumes a continuously varying volatility. More formally, in the simplest 
continuous framework, the evolution of prices is given by an Itô process:

$$
d \log \left(P_{t}\right)=\mu_{t} d t+\sigma_{t} d B_{t},
$$

with $P_{t}$ the random variable representing the price at time $t \in[0 ; T], \mu_{t}$ the drift term, $B_{t}$ the standard Brownian motion and $\sigma_{t}$ the instantaneous volatility.

The quantity of interest is here the Integrated Volatility at the time $t$, that is: $I V_{t}=\left(\int_{t-1}^{t} \sigma_{s}^{2} d s\right)^{\frac{1}{2}}$. The availability of tick-by-tick data then allows us to approximate the empirical Integrated Volatility by the Realized Volatility, which in turn is defined as such:

$$
\hat{\sigma}_{t}^{R V}=\left[\eta \sum_{i=1}^{I} \ln \left(\hat{P}_{t_{i}} / \hat{P}_{t_{i}-1}\right)^{2}\right]^{1 / 2},
$$

where $t=[0, \ldots, T]$ is the time stamp of the volatility computations (usually daily), $\left\{\hat{P}_{t_{i}}\right\}$, with $i=[1, \ldots, I]$, is the sequence of prices of the asset at time $t_{i}$ with $t_{i}=t-i / I$, the date of observation of return involved in the time-scale volatility, with $I$ being the time-resolution expressed in the number of observations per day, $\eta=n_{b}$ is a factor ${ }^{2}$ for annualizing the time-scale volatility where $n_{b}$ is the number of business days per year, and the notation $\hat{X}$ here standing for a realization (potentially measured with errors) of a random variable $X$.

Though this estimator converges in probability (in theory) towards the Integrated Volatility as the periodicity of observation goes down, some microstructure effects in practice skew the price dynamics, meaning that either the empirical estimation has to be done on a lower frequency than the maximum available or has to incorporate correcting terms, or both (see, among many others, Oomen, 2004 and 2005 for a discussion on this point).

Pushing further the analysis, Barndorff-Nielsen and Shephard (2004) introduced the so-called Realized Bi-power Variation, that writes (with the previous notations):

$$
\begin{aligned}
\hat{\sigma}_{t}^{R B V}=\left[\eta \sum_{i=2}^{I}\left|\ln \left(\hat{P}_{t_{i}} / \hat{P}_{t_{i}-1}\right)\right|\right. \\
\left.\times\left|\ln \left(\hat{P}_{t_{i}-1} / \hat{P}_{t_{i}-2}\right)\right|\right]^{1 / 2} .
\end{aligned}
$$

Thanks to the Realized Bi-power Variation definition, the Realized Variance can thus be split into two parts corresponding to its continuous and discontinuous (jump) components. The intuition is that while squaring the returns amplifies the jumps, the Realized Bi-power Variation, by multiplying each jump with the preceding or following return, dampens their overall effect. The difference between the Realized Volatility and the Realized Bi-power Variation will hereafter be called the Realized Jump Component and will be used herein to quantify the intensity of the jumps within one day.

\footnotetext{
${ }^{2}$ Note that, by contrast to Martens (2002), Areal and Taylor (2002) and Hol and Koopman (2002), we do not correct here the volatility for the presence of noisy overnight returns, since we do not focus on a special time-scale accurate measure, but rather on a specific time-series observation scale corresponding to economic agent observation frequency.
} 
When only few price observations are available daily, other definitions are needed. In most of financial studies only using low-frequency data, return dispersions are estimated by calculating the standard deviation of the time-series of daily, weekly, or monthly returns, based on security closing prices. If the risk was deemed a constant over the time, then the accuracy of the measure would increase with the number of points used in its computation. Since market risk fluctuates with time, using past observations in the current measure can actually be detrimental if these belong to a different distribution. One way to cope with the problem is to use a rolling window and compute the classical variance over a fixed period of time. Typical time periods vary from five business days to one year, depending on the investment horizon and actual use of the data (asset valuation, risk control, volatility trading...). The traditional proxy, called Simple (Close-to-Close) Volatility, is computed with daily data and writes:

$$
\hat{\sigma}_{t}^{S V}=\left\{\eta_{S V} \sum_{i=t-I+1}^{t}\left[\ln \left(\hat{P}_{i}^{C} / \hat{P}_{i-1}^{C}\right)-\hat{\mu}_{t}\right]^{2}\right\}^{1 / 2},
$$

where $I$ is the estimation window expressed in the number of business days, $t=[I-1, \ldots, T]$ are daily dates, $\eta_{S V}=n_{b} / I$ is the annualization correction parameter, $\left\{\hat{P}_{i}^{C}\right\}$ is a sequence of observed closing prices, and $\hat{\mu}_{t}$ is given by:

$$
\hat{\mu}_{t}=I^{-1} \sum_{i=t-I+1}^{t} \ln \left(\hat{P}_{i}^{C} / \hat{P}_{i-1}^{C}\right)
$$

which is an estimation of the mean log-return on the reference period.

This daily estimate will thus exhibit by construction a lot of serial dependence, since the same return observations enter into several consecutive volatilities. As pointed out by Poon and Granger (2003), since the statistical properties of sample mean make it a very inaccurate estimate of the true mean especially for a small sample, taking deviations around zero - or around a very long period mean - instead of a short sample mean, increases the accuracy of the estimate even if biased. Taking this reasoning to the extreme, the simplest measure of the Instantaneous (daily) Simple Volatility would be given by the squared return between two sampling dates (days), that is (with the previous notations):

$$
\hat{\sigma}_{t}^{I S V}=\left[\eta \ln \left(\hat{P}_{t}^{C} / \hat{P}_{t-1}^{C}\right)^{2}\right]^{1 / 2} .
$$

This measure is free of hypothesis on the mean return, and does not use overlapping information: we will thus use it as our proxy for instantaneous lowfrequency volatility in the rest of the paper. It is, however, extremely noisy, and not to be used in practical applications.

To alleviate some of the noise produced by this daily-based estimates, an Exponential Moving Average ${ }^{3}$ (EMA) is often applied. The EMA estimator is

\footnotetext{
${ }^{3}$ Sometimes called a RiskMetrics-type of measure.
} 
defined by induction by the following equation (with the previous notations):

$$
\begin{aligned}
\hat{\sigma}_{t}^{E M A}=\{\eta[ & \rho\left(\hat{\sigma}_{t-1}^{E M A}\right)^{2} \\
& \left.\left.+(1-\rho) \ln \left(\hat{P}_{t}^{C} / \hat{P}_{t-1}^{C}\right)^{2}\right]\right\}^{1 / 2},
\end{aligned}
$$

where $\rho$ is an ad hoc parameter governing the smoothness of the estimator ${ }^{4}$.

This classical standard deviation of return series is assumed to be a proxy for the dispersion of returns. Though it is easy to compute, it, however, ignores all the information concerning the path of the price inside the period of reference. Even at a low-(daily) frequency, though, additional information is often available in addition to closing prices, such as opening prices and highest and lowest prices within the day. Taking advantage of this information, Parkinson (1980) proposes an estimator of the variance based on the range of the random walk (defined as the difference between highest and lowest prices) during the day considered. He shows that the additional information provided by the high/low records of the period improves the accuracy of the estimation. Using the result by Feller (1951) on the joint distribution of the maximum and minimum of a random walk (assuming no drift and a constant dispersion denoted $\sigma$ ), we have:

$$
E\left[\ln \left(H_{t} / L_{t}\right)^{2}\right]=4 \ln (2) \sigma^{2},
$$

where $H_{t}$ and $L_{t}$ are the random variables corresponding to the highest and lowest prices on day $t$ :

$$
\left\{\begin{array}{l}
H_{t}=\operatorname{Max}\left\{P_{t_{i}} \mid t_{i} \in(t-1, t]\right\} \\
L_{t}=\operatorname{Min}\left\{P_{t_{i}} \mid t_{i} \in(t-1, t]\right\}
\end{array}\right.
$$

Parkinson (1980) defines his estimator as a function of the range that reads (with the previous notations):

$$
\hat{\sigma}_{t}^{P}=\left\{\eta_{P} \sum_{i=t-I+1}^{t}\left[\ln \left(\hat{H}_{i} / \hat{L}_{i}\right)\right]^{2}\right\}^{1 / 2},
$$

where $\eta_{P}=n_{b}[4 I \ln (2)]^{-1}$ is a correction parameter given by equation (7) which takes into account the number of estimations of the range.

The Parkinson (1980) extreme value estimator efficiency intuitively comes from the fact that the range of intra-daily quotes gives more information regarding the volatility than two arbitrarily spaced points in this series (the closing prices), for the low cost of two additional data points per day. Indeed, high and low prices are the results of continuous monitoring, instead of opening and

\footnotetext{
${ }^{4}$ It has been set to .5 (mild smoothing) hereafter, corresponding to a half-life of one day, and is usually set to .94 in the RiskMetrics measure (see "RiskMetrics Technical Document", Fourth Edition, J.P. Morgan, 1996, 296 pages). As explained in Moosa and Bollen (2002), with a decay factor of .94 , almost all relevant information is incorporated in the volatility using the last 60 trading days.
} 
closing prices which are defined for precise dates. Most financial data suppliers already provide daily high/low prices as summaries of intra-day activity. Roughly speaking, knowing these records allows us to get closer to the "real underlying process", even if we do not know the whole path of asset prices. Christensen and Podolskij (2007) show that such an estimate applies to a very large domain of price models. Moreover, this estimator is unbiased when the drift is equal to zero. Alizadeh et al. (2002), looking for a daily estimator, use directly the daily log-range. By assuming that the stochastic Log-volatility evolves as a mean-reverting Ornstein-Uhlenbeck process, they propose as an estimator (with the previous notations):

$$
\hat{\sigma}_{t}^{A B D}=\eta \exp \left[\ln \left(\hat{H}_{t} / \hat{L}_{t}\right)-\hat{\mu}\right],
$$

with $\hat{\mu}$ being equal to .43 in their sample.

They show that their estimator is both normally distributed and robust to microstructure noise. This result relies on the first moment of distribution of the log-range for a driftless Brownian motion given in Feller (1951).

But using now four data points - open, close, high and low prices - instead of only two - Close-to-Close or high-low prices - can also provides extra information - especially if opening and closing prices are closely watched by all market participants and act as references. Garman and Klass (1980) propose an estimator based on the knowledge of the open, close, high and low prices and the joint effects between this quantities. This estimator can be written (with the previous notations):

$$
\begin{aligned}
\hat{\sigma}_{t}^{G K}= & \left\{\eta_{G K}^{1} \sum_{i=t-I+1}^{t}\left[\ln \left(\hat{H}_{i} / \hat{L}_{i}\right)\right]^{2}\right. \\
& \left.-\eta_{G K}^{2} \sum_{i=t-I+1}^{t}\left[\ln \left(\hat{P}_{i}^{C} / \hat{P}_{i}^{O}\right)\right]^{2}\right\}^{\frac{1}{2}},
\end{aligned}
$$

where $I$ is the estimation window expressed in number of business days, $P_{i}^{C}$ is the random variable for the closing price at day, with $i=[0, \ldots, I], P_{i}^{O}$ is the random variable for the open price the same day, $\eta_{G K}^{1}$ and $\eta_{G K}^{2}$ are weighted correction parameters ${ }^{5}$, and the notation $\hat{X}$ standing for a realization (potentially measured with errors) of a random variable $X$.

This estimator is obtained as the one with the minimal variance among the analytic, scale invariant, unbiased estimators based on the four previously defined data points, and by using the fact that (with the previous notations):

$$
\begin{aligned}
r c l \sigma^{2} & =E\left[\ln \left(H_{t} / P_{t}^{O}\right)^{2}\right] \\
& =E\left[\ln \left(L_{t} / P_{t}^{O}\right)^{2}\right] \\
& =E\left[\ln \left(P_{t}^{C} / P_{t}^{O}\right)^{2}\right] \\
& =E\left\{\operatorname { l n } ( P _ { t } ^ { C } / P _ { t } ^ { O } ) \left[\ln \left(H_{t} / P_{t}^{O}\right)\right.\right. \\
& \left.\left.\quad+\ln \left(L_{t} / P_{t}^{O}\right)\right]\right\},
\end{aligned}
$$

${ }^{5}$ with $\eta_{G K}^{1}=n_{b} /(2 I)$ and $\eta_{G K}^{2}=n_{b}[1-2 \ln (2)] / I$. 
and:

$$
E\left[\ln \left(H_{t} / P_{t}^{O}\right) \ln \left(L_{t} / P_{t}^{O}\right)\right]=[1-2 \ln (2)] \sigma^{2} .
$$

Since Parkinson (1980) and Garman-Klass (1980) estimators implicitly assume that log-prices follow a geometric Brownian motion with no drift, and no opening jumps, further refinements are given by Rogers and Satchell (1991) and Kunitomo (1992). The latter author uses the open and close prices to estimate a modified range corresponding to an hypothesis of a Brownian bridge for the transformed log-price. The Kunitomo (1992) estimator basically aims to correct the high and low prices for the presence of a drift. The formula is based on extremes of a constructed Brownian bridge motion with constant volatility that gives (with the previous notations):

$$
E\left(h_{t} / l_{t}\right)=\left(\pi^{2} / 6\right) \sigma^{2},
$$

where:

$$
\left\{\begin{aligned}
h_{t}= & \operatorname{Max}\left\{\ln \left(P_{t_{i}} / P_{t}^{O}\right)\right. \\
& \left.\quad-\left(t_{i}-t-1\right) \ln \left(P_{t}^{C} / P_{t}^{O}\right) \mid t_{i} \in(t-1, t]\right\} \\
l_{t}= & \operatorname{Min}\left\{\ln \left(P_{t_{i}} / P_{t}^{O}\right)\right. \\
& \left.-\left(t_{i}-t-1\right) \ln \left(P_{t}^{C} / P_{t}^{O}\right) \mid t_{i} \in(t-1, t]\right\} .
\end{aligned}\right.
$$

Kunitomo (1992) then proposes the following unbiased estimator of the previous quantity (with the previous notations):

$$
\hat{\sigma}_{t}^{K}=\left[\eta_{K} \sum_{i=t-I+1}^{t}\left(\hat{h}_{i}-\hat{l}_{i}\right)^{2}\right]^{1 / 2},
$$

where $\eta_{K}=6 n_{b} /\left[I \pi^{2}\right]$ is a correction parameter given by equation (13), taking into account the number of observations.

This formula implies then that enough tick-by-tick trading data are available. In the limit case, it means in fact that the full discrete Brownian motion is known, which makes the above estimator useless.

Rogers and Satchell (1991) also add a drift term in the stochastic process that can be incorporated into a volatility estimator using only daily open, high, low, and closing prices, that gives the following unbiased estimator (with the previous notations):

$$
\begin{aligned}
\hat{\sigma}_{t}^{R S}= & {\left[\eta _ { R S } \sum _ { i = t - I + 1 } ^ { t } \left\{\operatorname { l n } ( \hat { H } _ { i } / \hat { P } _ { i } ^ { O } ) \left[\ln \left(\hat{H}_{i} / \hat{P}_{i}^{O}\right)\right.\right.\right.} \\
& \left.-\ln \left(\hat{P}_{i}^{C} / \hat{P}_{i}^{O}\right)\right]+\ln \left(\hat{L}_{i} / \hat{P}_{i}^{O}\right)\left[\ln \left(\hat{L}_{i} / \hat{P}_{i}^{O}\right)\right. \\
& \left.\left.\left.-\ln \left(\hat{P}_{i}^{C} / \hat{P}_{i}^{O}\right)\right]\right\}\right]^{1 / 2},
\end{aligned}
$$

with $\eta_{R S}=n_{b} /(I+1)$ the annualization correction factor.

This estimator exhibits a lower variance compared to the one of the Parkinson (1980) estimator. 
They also propose an adjustment that is designed for taking into account the fact that one may not be able to continuously monitor the stock price, and then the exact minimum and maximum prices. Their adjusted estimator $\hat{\sigma}_{t}^{A R S}$ is the positive root of the following quadratic equation (with the previous notations):

$$
\begin{aligned}
\left(\eta_{1}^{*} / V_{t}\right) \hat{\sigma}_{t}^{A R S}+\left(\eta_{2}^{*} / \sqrt{V_{t}}\right) \ln & \left(\hat{H}_{t} / \hat{L}_{t}\right) \hat{\sigma}_{t}^{A R S} \\
& +\left(\hat{\sigma}_{t}^{R S}\right)^{2}=0
\end{aligned}
$$

with $V_{t}$ is the total number of transactions occurring during day $t$, and the constants being $\eta_{1}^{*}=.5594$ and $\eta_{2}^{*}=.9072$.

Finally, Yang and Zhang (2000) make further refinements by deriving an Extreme Value estimator that is unbiased, independent of any drift, and consistent in the presence of opening price jumps. They look for an unbiased estimator written through a quadratic expression on the open, close, high and low prices of different periods. With symmetry arguments, they prove that any unbiased estimator, independent of the drift and the opening jump, must write as a weighted average of the Rogers and Satchell (1991) estimator, the close-open volatility and the open-close volatility, i.e. (with the previous notations):

$$
\hat{\sigma}_{t}^{Y Z}=\left[\left(\hat{\sigma}_{t}^{O}\right)^{2}+\kappa\left(\hat{\sigma}_{t}^{C}\right)^{2}+(1-\kappa)\left(\hat{\sigma}_{t}^{R S}\right)^{2}\right]^{1 / 2},
$$

with:

$$
\left\{\begin{array}{l}
\left(\hat{\sigma}_{t}^{O}\right)^{2}=\eta_{Y Z} \sum_{i=t-I+1}^{t}\left[\ln \left(\hat{P}_{i}^{O} / \hat{P}_{i-1}^{C}\right)-\overline{\ln \left(\hat{P}_{i}^{O} / \hat{P}_{i-1}^{C}\right)}\right]^{2} \\
\left(\hat{\sigma}_{t}^{C}\right)^{2}=\eta_{Y Z} \sum_{i=t-I+1}^{t}\left[\ln \left(\hat{P}_{i}^{C} / \hat{P}_{i}^{O}\right)-\overline{\ln \left(\hat{P}_{i}^{C} / \hat{P}_{i}^{O}\right)}\right]^{2},
\end{array}\right.
$$

where $\eta_{Y Z}=n_{b} /(I-1)$ is the annualization correction parameter and the notation $\bar{X}_{i}$ is here used for the unconditional mean of the sequence of the variable $X_{i},\left(\hat{\sigma}_{t}^{R S}\right)^{2}$ is the Rogers-Satchell (1991) estimator (see above text) and $\kappa$ is a specific constant.

This last constant $\kappa$ is chosen in order to minimize the variance of the estimator, and so is the solution of a quadratic problem. Assuming that $\sigma_{t}^{O}$ is independent of $\sigma_{t}^{C}$ and $\sigma_{t}^{R S}$, and that $\sigma_{t}^{C}$ and $\sigma_{t}^{R S}$ are uncorrelated, it writes (with the previous notations):

$$
\kappa=\frac{E\left\{\left[\left(\sigma_{t}^{O}\right)^{2}+\left(\sigma_{t}^{R S}\right)^{2}\right]\left[\left(\sigma_{t}^{R S}\right)^{2}-\left(\sigma_{t}^{C}\right)^{2}\right]\right\}}{E\left\{\left[\left(\sigma_{t}^{R S}\right)^{2}-\left(\sigma_{t}^{C}\right)^{2}\right]^{2}\right\}},
$$

which leads to the following general expression, taking into account the number of points denoted $I$, such as:

$$
\hat{\kappa}=(\zeta-1)[\zeta+(I+1) /(I-1)]^{-1},
$$


for some constant ${ }^{6} \zeta$.

To summarize the presentation of the previous estimators, we can distinguish them with respect to the number and to the type of points entering in their definition. With only close prices, the Simple Volatility and the EMA are computed with one data per day. Based on the daily range, the Parkinson estimator is the simplest estimator to compute with the highest and lowest daily prices. The Garman-Klass and the Rogers-Satchell estimators outperform their estimator characteristics by taking into account the opening and closing prices, as well as the Yang-Zhang one, which furthermore incorporates the overnight information in its definition using the closing price of the previous day. Finally, Kunitomo estimator, using Brownian bridge properties, the Realized Volatility and the Realized Bi-power Variation have as a common feature that they call on all daily high frequency data. Moreover, all estimators are characterized by a specific correction parameter which is obtained by imposing the estimators to be without bias, with minimal variance and to satisfy some other hypotheses on the underlying stochastic process that represents the dynamics of prices. Saichev et al. (2009 and 2010) recently propose a more general approach of all estimators based on the four prices (open, high, low and close) in order to choose, through adapted diagrams, the most efficient estimators with respect to the statistical properties of the series of log-prices, which does not need to be Gaussian anymore. For illustration purposes, they apply their method for selecting the best estimator among the Parkinson, Rogers-Satchell, Garman-Klass and Yang-Zhang estimates.

The range-type of volatility estimator is a highly efficient volatility proxy as shown by Brandt and Diebold (2006) in a multivariate setting. In turbulent days with drops and recoveries of the markets, the traditional Close-to-Close volatility can still be low while the daily range correctly indicates that the volatility is high. Furthermore, the range appears robust to microstructure biases such as the bid-ask bounce. As indicated by Brandt and Diebold (2006), the observed daily maximum is in general reached at the ask price and hence is too high by half the spread, whilst the observed minimum is likely to be attained at the bid price and therefore is too low by half the spread. Generally speaking, the bid-ask bounce inflates the range only by the average spread, which is, in general, in liquid markets small, relative to the price moves. The price range is, however, inefficient when compared to high-frequency estimators (see Andersen and Bollerslev, 1998-a, p.898, footnote 20, and Andersen et al., 2003) in the Brownian motion case. But it is far from clear that range-based estimators are less efficient outside the Brownian case study or in the presence of market microstructure effects, simply because the problem is difficult to handle theoretically and because microstructure noise covers a large collection of potential biases which are difficult either to properly define or explain (see Crack and Ledoit, 1996).

However, consistent with the previous research of Wiggins (1991), Li and

\footnotetext{
${ }^{6}$ Yang and Zhang (2000) show theoretically and with numerical calculations that $\zeta$ belongs to the range ]1.33; $1.50[$, depending on the value of the drift. They, however, suggest to fix $\zeta$ equal to 1.34 in practice.
} 
Weinbaum (2001) find that the Parkinson estimator - applied to the daily S\&P 100 and S\&P500 data covering periods of 1 to 24 (trading) days over the period from January 1989 to December 1999 - is downward biased compared to the traditional estimator, at the weekly and monthly frequencies. This is also true for the other extreme value volatility estimators. They provide rational to explain this result and other coherent Extreme Value Volatility estimates on S\&P500 Futures and exchange rate markets (see Li and Weinbaum, 2001, p.19 and following). Furthermore, Corrado and Miller (2006) conclude that when used in Merton (1980) Reward-to-Risk model of expected excess return, most of the extreme estimators of volatility lead to similar qualitative results. Corrado and Truong (2007) also demonstrate that the intra-day High-Low estimator price range offers a volatility forecast with an efficiency similar to Implied Volatility as a predictor of future volatility, as well as Bali and Weinbaum (2005) who show that most of the Extreme Value Volatility estimators are better measures of the Realized Volatility than the Simple Volatility for short time-horizons (mainly weekly and monthly). Extreme Value Volatility estimators finally seem to be highly correlated one to another and strongly linked to the implied and future volatilities.

In terms of efficiency (measured as the ratio of the variance of the Extreme Value estimator over the Close-to-Close one), all previous estimators exhibit very substantial improvements. As reported in Corrado and Miller (2006), the Parkinson (1980) measure shows a theoretical relative efficiency gain ranging from 2.5 to 5, while the Garman and Klass (1980), Yang and Zhang (2000) and Kunitomo (1992) variance estimators result in a theoretical efficiency gain of, respectively, 7.4, 7.3, and 10. Rogers et al. (1994) report that the Rogers-Satchell (1991) estimator yields theoretical efficiency gains comparable to the GarmanKlass estimator. They also report that the Rogers-Satchell (1991) estimator performs well with changing drifts and as few as 30 daily observations. Bali and Weinbaum (2005) confirm this general conclusion regarding the efficiency of Extreme Value estimators, using both an American stock Index (S\&P500 future contracts) and, as a double-check, a main exchange rate database, on various short-term horizons.

\section{Extreme Values of Risk Estimates}

When studying volatility distribution, the main area of interest is the righthand tail where the highest volatilities are located. The following sections are accordingly devoted to the exploration of the rightmost part of the distribution function in order to highlight the differences between the estimators. In fact, few studies have been devoted to the distribution itself of the volatility. They, however, almost all conclude that volatilities are Lognormally distributed, as in Taylor (1986) and Andersen et al. (2001-a). Going further, Liu et al. (1999), show that if the Log-normal distribution describes well the central part of the distribution of volatilities, this statement, however, appears less positive in the tail, since, in fact, a power law asymptotic behavior seems to be more adequate. 
Following this remark and given the descriptive statistics, we will then focus on the so-called Tail-index $\gamma$, equal to the inverse of the Shape-parameter, denoted $\xi$, of the distribution of the volatility $\sigma$, which characterizes, when positive, an heavy-tailed density. This kind of approach is already well developed concerning the distribution of returns. Indeed, since Fama (1965), it is known that the distribution of returns does not follow a Gaussian distribution, which is recognized as an undisputed stylized fact (see Cont, 2001). Longin (1996 and 2005) proposes to also use the Tail-index in order to discriminate among the distributions of returns.

Among the various possible approaches to estimate the Tail-index, three are presented hereafter: the Non-parametric "Upper Order Statistics" (UOS) in sub-section 3.1, the Parametric "Block Maxima" (BM) for GEV distribution of extrema in sub-section 3.2 and the Parametric Peaks-over-the-Threshold (PoT) Method for the GPD in sub-section 3.3.

In fact, the choice of the GEV or GP Distributions for getting a more accurate characterization of the distribution of extremes is indeed complementary. Both density functions have the same asymptotic behaviors and share the same Tail-index. In other words, as explained in Coles (2001) for instance, the parameters of the Generalized Pareto Distribution of threshold excesses are uniquely determined by those of the associated GEV distribution of Block Maxima. In particular, the Shape-parameter is equal to that of the corresponding GEV distribution. When for instance it is assumed that the process by which the random variable exceeds the threshold is a Poisson process, Coles (2001) and Katz et al. (2005) prove that the three characteristic parameters of the GPD (see below) are directly linked to those of a GEV. ${ }^{7}$ With the same approach, Ding et al. (2008) obtain an approximate relation between the Quantile Functions of GEV distributions and the GPD. They first conclude that GEV distributions can be seen as special cases of GPD, especially for high thresholds, and they, secondly, empirically observe that the GPD has more accurate fitting properties than the GEV distribution. Moreover, Bali (2003-a and 2003-b) proposes, using a Box and Cox (1964) transformation, a Generalization of the GEV density that also encompasses the pure GEV and GPD as special cases.

For these reasons, we then expect in the following that real market price related estimations of volatility Tail-indexes to be very close whatever the density and the method of estimation.

\footnotetext{
${ }^{7}$ If we note $v$ and $\alpha$ the location-dispersion characteristic parameters of a GPD and $v^{*}, \alpha^{*}$ and $\xi^{*}$ the three parameters of the related GEV distribution (see below), then the Poisson process, defined by the parameter $\lambda \in \mathbb{R}_{+}^{*}$, that governs the crossing rate for the Peaks-overThreshold, allows us to write the following relations for the location and dispersion parameters such as:$$
\left\{\begin{array}{l}
v=v^{*}-\frac{\alpha^{*}}{\xi^{*}}\left(\lambda^{*}-1\right) \\
\alpha=\alpha^{*}-\xi^{*}\left(v-v^{*}\right)
\end{array}\right.
$$ 


\subsection{Extreme Risk Values using Non-parametric Upper Or- der Statistics}

One of the most popular ways of retrieving the Tail-index (corresponding to a large non-parametric class of distributions sharing some heavy-tail properties) is to use a non-parametric estimator, focusing a part of the highest volatility under studies (UOS approach). Before giving examples of such estimators, we first recall, following Resnick (1997), that a distribution function $F($.$) admits$ heavy tails if it satisfies, when $\sigma$ - the variable of interest - tends to infinity, for some $\xi \in \mathbb{R}_{+}$, the following relation:

$$
1-F(\sigma)=\sigma^{-1 / \xi_{\mathfrak{K}}(\sigma),}
$$

where $\mathfrak{K}($.$) is a slowly-varying function { }^{8}$ satisfying, for every $\sigma>0$ :

$$
\lim _{\triangle \rightarrow+\infty}[\mathfrak{K}(\triangle \sigma) / \mathfrak{K}(\triangle)]=1 .
$$

This definition corresponds to a Pareto-type tail behavior. The parameter $\xi$ is called the Shape-parameter (which is the inverse of the Tail-index, also called extreme-value index in some articles). A consequence of this definition of an heavy-tailed distribution is that it does not possess a complete set of moments, since $E\left[\sigma^{k}\right]$ is infinite for some values of $k$, with $k=[1, \ldots, n]$, when $\xi$ is positive. More precisely, $E\left[\sigma^{k}\right]=\infty$ whenever $k>1 / \xi$ and $E\left[\sigma^{k}\right]<\infty$ for $k<1 / \xi$ (Resnick, 1997). By transforming the previous equation with logarithms, we have:

$$
\ln [1-F(\sigma)]=\left(-\xi^{-1}\right) \ln (\sigma)+\ln [L(\sigma)] .
$$

Let $\left\{\sigma_{[i: N]}\right\}$, with $i=[1, \ldots, N]$, being the order statistic of order $i$ of the volatility $\sigma$, with $N$ the total number of observations, i.e. $\sigma_{[1: N]} \leq \sigma_{[i: N]} \leq$ $\sigma_{[N: N]}$. The value of $\left[1-F\left(\sigma_{[i: N]}\right)\right]$ can be estimated by the frequency of order statistics larger than $\sigma_{[i: N]}$, which is by definition equal to $(N-i+1) / N$. Then, by plotting on one axis $\left\{\ln \left(\sigma_{[i: N]}\right)\right\}$, and on the other one $\{\ln [(N-i+1) / N]\}$, for $i=[1, \ldots, N]$, the previous equation characterizes a straight line, with a slope which is $-\xi^{-1}$ (the opposite of the Tail-index). Coming back to the equation (21), we then observe in this case that only two points are theoretically sufficient to estimate the slope. Hence, the most natural estimator is the one proposed by de Haan and Resnick (1980), such as:

$$
\hat{\xi}_{H R_{(k, N)}}=-\left[\frac{\ln \left(\sigma_{[k: N]}\right)-\ln \left(\sigma_{[N: N]}\right)}{\ln (N-k+1)-\ln (1)}\right] .
$$

The numberk of data points chosen has not to be too small, in order to stay in the tail region, but not so high since it involves distortions due to few observations. For example, DuMouchel (1983) proposes to consider $k$ such as

\footnotetext{
${ }^{8}$ Classical examples of such functions are: $\mathfrak{K}()=$.$k with k$ a constant, $\mathfrak{K}()=.\ln ($.$) or$ $\mathfrak{K}()=.\ln [\ln ()$.$] .$
} 
$(N-k) \leq .1 \times N$ and a discussion on this matter can be found in Embrechts $e t$ al. (1997).

This method is thus a simple version to the OLS Log-log rank-size regression, which takes into account all observations in order to estimate the slope of the line. Moreover, in order to improve this last method, Gabaix and Ibragimov (2009) recently propose to use a translation of $-1 / 2$ on the rank, which optimally reduces the bias of the estimation. Applied to the previous formulae, it gives then:

$$
\hat{\xi}_{H R G I_{(k, N)}}=-\left[\frac{\ln \left(\sigma_{[k: N]}\right)-\ln \left(\sigma_{[N: N]}\right)}{\ln (N-k+1 / 2)-\ln (1 / 2)}\right] .
$$

There are other ways to estimate this slope, by considering either another couple of points or a larger set of points in the tail area (see Kearns and Pagan, 1997). Pickands (1975) defines an estimator by using Quantile Functions, and their estimates, which applies to the GEV distribution and the GPD, that writes (with the previous notations):

$$
\hat{\xi}_{P_{(k, N)}}=\ln (2)^{-1} \ln \left(\frac{\sigma_{[N-k / 4: N]}-\sigma_{[N-k / 2: N]}}{\sigma_{[N-k / 2: N]}-\sigma_{[N-k: N]}}\right),
$$

altogether with a method for choosing an optimal $k$.

In the same period, Hill (1975) proposes the following popular estimator:

$$
\hat{\xi}_{H_{(k, N)}}=k^{-1} \sum_{i=1}^{k}\left[\ln \left(\sigma_{[N-i+1: N]}\right)-\ln \left(\sigma_{[N-k: N]}\right)\right] .
$$

The Hill (1975) estimator is shown to be more accurate than the Pickands (1975) alternative, but is, however, only designed for the estimation of positive extreme value indexes. A mathematical justification of the Hill estimator lies in the simple fact that the sample $\left\{\ln \left(\sigma_{[N-i+1: N]} / \sigma_{[N-k: N]}\right)\right\}$, with $i=[1, \ldots, k]$, has a marginal density close to an Exponential density with parameter $\xi^{-1}$. A classical result states that the Maximum Likelihood estimator of $\hat{\xi}$, for an Exponential distribution, is the simple mean, which is in this case equal to $\hat{\xi}_{H_{(k, N)}}$.

In practice, the Hill estimator $\hat{\xi}_{H_{(k, N)}}$ is plotted against some $k$ and, if a consistent stable region appears in the graph, then $\xi$ will be estimated by the value of $\hat{\xi}_{H_{(k, N)}}$ in this region.

Later, Dekkers et al. (1989) propose an extension to the Hill estimator, called Moment Estimator, which takes into account its second moment such as (with the previous notations):

$$
\begin{aligned}
\hat{\xi}_{D_{(k, N)}}=\hat{\xi}_{H_{(k, N)}}+1 & \\
-2^{-1} & \left\{1-\hat{\xi}_{H_{(k, N)}}^{2}\left[M_{(k, N)}\right]^{-1}\right\},
\end{aligned}
$$

where:

$$
M_{(k, N)}=k^{-1} \sum_{i=1}^{k}\left[\ln \left(\sigma_{[N-i+1: N]}\right)-\ln \left(\sigma_{[N-k: N]}\right)\right]^{2} .
$$


This estimator is asymptotically normal under very general conditions given in Dekkers et al. (1989), and can be applied to any value of the Tail-index of an Extreme Value distribution.

Challenging the well-known inaccuracy of the Hill (1975) estimator, Beirlant et al. (1996) proposed an extension of this estimator, valid for any value of the Tail-index of an Extreme Value distribution. This extension reads (using the previous notations):

$$
\begin{aligned}
\hat{\xi}_{G H_{(k, N)}}=\hat{\xi}_{H_{(k, N)}}+k^{-1} \sum_{i=1}^{k} & {\left[\left(\ln \hat{\xi}_{H_{(i, N)}}\right)\right.} \\
& \left.-\ln \hat{\xi}_{H_{(k+1, N)}}\right] .
\end{aligned}
$$

The first term in the previous equation is the classical Hill (1975) estimator with the deletion of the maximum of the series, and the second term is computed on the classical Hill estimators themselves. As in the cases of Hill and Pickand estimators, the value of the non-parametric Generalized Hill Tail-index estimation will depend upon the nuisance parameter - the number of data points $k$ considered in the computation, but is shown to be more robust than simple estimates (see Kearns and Pagan, 1997; Caers et al., 1998). Here also, the measure must be read on a graph, eyeballing some convergence to a certain value, while keeping the number of observations relatively low, so as not to take points from the center of the wings of the underlying distribution.

Finally, Huisman et al. (2001) have also developed a modified Hill estimator in order to reduce its bias for small samples. Their estimator is obtained with an OLS regression on $k$, the number of points in the tail, and can then be applied only for positive Shape-parameters. As underlined by LeBaron (2009), this estimator also appears to be accurate enough (in the sense of the Mean Square Error) to get precise Tail-index estimates.

Hence, the previous Shape-parameter estimates can be divided into two classes. The first group concerns the estimators based only on Pareto-type tail behavior, and can then only be applied for positive Shape-parameters: are here concerned the de Haan and Resnick, Hill, and the Huisman et al. estimates. In the second group, Pickands, Dekkers et al. and Beirlant et al. estimates are valid, whatever the sign of the Tail-index, provided that an Extreme Value distribution assumption is satisfied. Furthermore, Dekkers et al. (1989) give some sufficient and necessary conditions in order to explicitly link a Pareto-type tail behavior to a specific Extreme Value Distribution.

\subsection{Extreme Risk Values using the Block Maxima Method for Generalized Extreme Value Density Estimation}

According to the central result of Extreme Value Theory, the extrema of the measures of the risk, if IID, should converge asymptotically to the Generalized Extreme Value (GEV) distribution ( $C f$. Jenkinson, 1955). The GEV distribution is characterized by three parameters: $v \in \mathbb{R}$, the location parameter, $\alpha \in \mathbb{R}_{+}^{*}$, the scale parameter and $\xi \in \mathbb{R}$ known as the Shape-parameter (the 
inverse of the Tail-index). This last parameter measures the rate of decrease of the probability in the tails, such as the Jenkinson limiting non-degenerate distribution of an IID normalized minimum. Taking into account that our variable of interest - the volatility - is non-negative, the Cumulative Density Function of a GEV distribution of Log-volatility block maxima, denoted here $\sigma$ for the sake of simplicity, is given by:

$$
H(\sigma)=\left\{\begin{array}{l}
\exp \left\{-\left[1+\xi_{\alpha}^{-1}(\sigma-v)\right]^{-1 / \xi}\right\} \text { if } \xi \neq 0 \\
\exp \left\{-\exp \left[-\alpha^{-1}(\sigma-v)\right]\right\} \text { otherwise }
\end{array}\right.
$$

for every $\sigma \in \mathcal{D}_{1}$, defined by:

$$
\mathcal{D}_{1}= \begin{cases}]-\infty ; v-\xi_{\alpha}[ & \text { if } \xi<0 \\ \mathbb{R} & \text { if } \xi=0 \\ ] v-\xi_{\alpha} ;+\infty[ & \text { if } \xi>0\end{cases}
$$

with $\xi_{\alpha}=\alpha / \xi$.

Three basins of attraction can be distinguished according to the value of the Shape-parameter $\xi$ : positive, zero or negative. In the case of fat-tailed distributions (Gnedenko Limiting distribution of Fréchet for the Extremes and a leptokurtic distribution for the underlying Log-Volatilities), the Shape-parameter will be significantly positive, whereas the Gaussian underlying distribution yields a null Shape-parameter (corresponding to the Gumbel distribution for the Extremes and the Normal for Log-volatilities). The third case, where the Shapeparameter is negative, corresponds to the Weibull-kind of distributions for Extremes.

The Extreme Value Theory in its original definition deals with independent observations, whereas the persistence of variance is a well known phenomenon in finance, meaning the extremes will occur in clusters. Nevertheless, providing that the dependence is "weak", the previous results hold in the sense that the resulting distribution is also a GEV density, intensified by a power real - called the Extremal Index, denoted $\theta$, with $\theta=[0 ; 1]$, defined as such:

$$
H(\sigma)=\left[H\left(\sigma^{*}\right)\right]^{\theta},
$$

where $\xi$ is the Shape-parameter, $\sigma$ the non-IID variable with clusters and $\sigma^{*}$ an associated IID variable whose GEV limiting distribution shares the same Tail-index (see Embrechts et al., 1997 and Jondeau and Rockinger, 2003, for more details on the Extremal Index).

This means that the asymptotic distribution of (normalized) Maxima for the non-IID series is in fact a GEV distribution with parameters we would have had if the series were IID, but raised to the power $\theta$. It can be understood as the extremes over sub-samples of size $n$ (for $n=5,20$ or 60 in our case) for the non-IID series have the same behavior that the extremes over sub-samples of size $n \theta$ for the related IID series. In particular, this means that the value $\theta=1$ corresponds to independence or weak dependence cases. Using the Block Maxima Method ( $C f$. Embrechts et al., 1997), the Extremal Index $\theta$, interpreted 
as the reciprocal of the mean cluster size, can be estimated asymptotically using the following expression (Hsing et al., 1988, and Hsing, 1991):

$$
\hat{\theta}=n^{-1} \ln \left(1-K_{\alpha} / m\right)\left[\ln \left(1-N_{\alpha} / m n\right)\right]^{-1},
$$

where $N_{\alpha}$ is the number of volatility exceeding some threshold $\alpha, K_{\alpha}$ is the number of blocks in which this threshold is exceeded, and $m$ and $n$ are, respectively, the arbitrary number of blocks and related length of these blocks ${ }^{9}$. Compared to the results by Jondeau and Rockinger (2003) who report mean values of Extremal Indexes ranging from .71 to .93 for the twenty main world stock market indice return series using this technique, we find in our sample of Log-volatilities a mean value for the Extremal Index of .72 (ranging from .59 to .87 for all volatility estimators) for a probability threshold fixed at $99.00 \%$ and for considered weekly blocks (see Figure 1-d below) obtained on 10,000 extracted sub-samples of 500 daily volatilities; this indicates a rather strong clustering phenomenon ${ }^{10}$ and, thus, an overall potential important impact on Tail-index estimates of the non-IDD characteristic of the underlying volatility series.

However, pure GEV distributions for IID and non-IID series theoretically share the same Tail-index, because rising to a power the GEV distribution only affects its scale and location parameters (see Byström, 2004). Since focusing on the extreme tail of the unconditional large volatilities, we should a priori not be worrying too much about the non-IID characteristics of such a financial feature, provided that the mis-specification risk is controlled. However, some empirical experiments are yet to conclude to the possible impact on the non-respect of the IID hypothesis (Kearns and Pagan, 1997; Malevergne et al., 2006). Indeed, when the GEV density does not perfectly fit the data and in a context of parameter uncertainty, the non-respect of the IID hypothesis imposes to take some precautions when interpreting results, due to some potential misspecification errors. The asymptotic derivation of the Extremal Index suggests that we should attempt to keep in applications both the number of blocks $(m)$ and the length of these blocks $(n)$ large, for a reasonable number of maxima (see McNeil, 1998). Still, Coles (2001) suggests that the Block-Maxima method - considering sub-samples longer than the potential serial dependency for estimating GEV densities - should reduce the impact on the parameter uncertainty. However, Malevergne et al. (2006) show, based on extended simulations, that two opposite effects are competing when studying the extrema. On the one hand, as mentioned above, the dependence structure of a variable creates a downward bias with respect to the IID case (mainly because the clustering of volatilities prevents, in small samples, the full exploration of the "true" extreme of the tail). On the other hand, the lack of convergence of the distribution towards the GEV asymptotic distribution results in an upward bias; the latter effect is

\footnotetext{
${ }^{9}$ See also Robert et al. (2009) who have recently proposed a new estimation method of the Extremal Index, based on sliding blocks, instead of fixed disjoint blocks.

10 which also decreases when the block maxima window is enlarged (mean indexes equal to .49 and .37 for monthly and quarterly lenghts) and when the threshold is lower (mean indexes are equal to .61 and .54 for thresholds fixed at $95.00 \%$ and $90.00 \%$ ).
} 
strengthened by the existence of a time-dependence, reducing the "effective" size of the sample. Using the Block Maxima method, partly destroying the dependence structure but hiding some extrema, allows us to focus more on the extremes and should thus limit the impact of the non-IID effect on estimations.

As a result of the previous discussion, we run several robustness checks in the following empirical applications (see Section 4 below), in order to make sure that our results are not only driven by a mis-specified conditional Extreme Value distribution. We first vary the maxima lengths (weekly, monthly and quarterly) and, secondly, test in each case (as recommended by Gonzalo and Olmo, 2004), whether the GEV density results in a significant KolomogorovSmirnov Goodness-of-Fit statistic. We thirdly conduct the same estimation exercise on data-sampled artificial return series (see below). We also repeated the exercise of estimation of Tail-indexes of volatility densities using the PoT method for fitting a GPD, since shown to be more robust, as advised by Coles (2001), Malevergne et al. (2006), Diebolt et al. (2008), and Ding et al. (2008), and as presented hereafter.

\subsection{Extreme Risk Values using the Parametric Peaks-over- Threshold Method for Generalized Pareto Distribu- tion Estimation}

To obtain a measure of the rate of decrease of the distribution function in its tail, an alternative method to the Block Maxima Method is to use not a sample of the Maxima, but all Peaks-over-Threshold (PoT Method), that are values of the random variable that exceed a certain cut-off point. In that case, the asymptotic distribution is a Generalized Pareto Distribution (GPD) which has been developed by Balkema and de Haan (1974), and Pickands (1975). Diebolt et al. (2008) note that this distribution is statistically more accurate compared to the GEV distribution when all observations of a sample are available. The GPD is characterized by three parameters: $v \in \mathbb{R}$, the location parameter, $\alpha \in$ $\mathbb{R}_{+}^{*}$, the scale parameter and $\xi \in \mathbb{R}$ known as the Shape-parameter. Taking into account that the volatility is non-negative, the Cumulative Density Function of a GPD of the Log-volatility exceedances, also denoted here $\sigma$ for the sake of simplicity, is given by:

$$
H(\sigma)= \begin{cases}1-\left[1+\xi_{\alpha}^{-1}(\sigma-v)\right]^{-1 / \xi} & \text { if } \xi \neq 0 \\ 1-\exp \left[-\alpha^{-1}(\sigma-v)\right] & \text { otherwise }\end{cases}
$$

for every $\sigma \in \mathcal{D}_{2}$, defined by:

$$
\mathcal{D}_{2}= \begin{cases}] v ; v-\xi_{\alpha}[ & \text { if } \xi<0 \\ ] v ;+\infty[ & \text { if } \xi \geq 0\end{cases}
$$

with $\xi_{\alpha}=\alpha / \xi$.

This distribution is a generalization in the sense that it subsumes certain other distributions under a common parametric form. Most of the common 
continuous distributions of statistics belong to this general class of distributions. If $\xi>0$, then it corresponds to heavy-tailed distributions, such as the Student for example, and the GPD is a reparametrized version of the ordinary Pareto (type I) Distribution, whilst the case where $\xi=0$ corresponds to the Exponential Distribution alike the Gaussian or the Lognormal whose tails decay exponentially. The situation where $\xi<0$ is linked to short-tailed distributions with a finite endpoint, such as the Uniform (for $\xi=-1$ ) or Beta, known as the Pareto type II Distribution family. von Mises (1936) gives necessary and sufficient conditions for a distribution to belong to the domain of attraction (defined by the Fisher-Tippett theorem, 1928) of one of the three extreme value distributions, which entails an immediate relation between the GEV distribution and the GPD. One then ascertains that the Normal distribution and all monotone transformations of the Normal distribution belong to the Gumbel domain of attraction, that the Pareto, Cauchy and Student laws belong to the Fréchet domain of attraction ${ }^{11}$, and that the Beta and Uniform distributions are in the Weibull's.

An important issue when implementing the PoT method is the cut-off to be chosen. If we are interested in the $5 \%$ quantile, in a VaR context for example, then the chosen threshold must be low enough to be able to estimate the interesting quantile, but not too far since otherwise occurrences truly belonging to the center of the distribution might contaminate the estimation of extremes. Indeed, the choice of the optimal threshold is a delicate issue since we are facing a bias-variance trade-off. Theory tells us that the threshold to be chosen should be high enough, but the higher the threshold, the less observations are left for the estimation and a small sample problem with slow convergence of the tail appears. The issue of determining the fraction of data belonging to the tail has been treated by several authors (see Hall, 1990; Danielsson and de Vries,1997 and 2000; Draisma et al., 1999; Danielsson et al., 2001; Gomes and Oliveira, 2001; Caeiro and Gomes, 2008; among others). However, they do not provide a definitive answer to the question of which method should be used. But we know that if we choose a too low threshold, we might recover biased estimates because the limit theorems for the GPD do not apply anymore, whilst selecting high thresholds generate estimates with high standard errors due to the limited number of observations. Preferring high thresholds may however limit the potential impact of non-IID variable on the mis-specification of the selected Extreme Value density, deleting some high volatilities (just below the threshold) from the studied sample, but may as well just intensify the problem since the persistence is also stronger for large deviations. At minimum, heteroskedasticity and volatility persistence may in our case, as in the case of the BM method with a GEV density, cause some perturbations in the estimations, even if showed more limited in the case of a GPD fit as highlighted by Malevergne et al. (2006).

To make more certain that our results in the following applications are not driven by a mis-specified conditional Extreme Value distribution (see Section 4

\footnotetext{
${ }^{11}$ For example, the Student-t can be related to a Fréchet distribution with a Shapeparameter equal to its degree of freedom.
} 
below), we once again run several robustness checks. We first vary the ad hoc peak thresholds $(2.5 \%, 5 \%$ and $10 \%)$ and, secondly (following the argument by Gonzalo and Olmo, 2004), we test in each case whether the GPD provides a significant Kolomogorov-Smirnov Goodness-of-Fit statistic. ${ }^{12}$ We thirdly rely on some extra data-sampled artificial returns (bootstrap and surrogate series) for estimating the GPD under various sample transformation hypotheses (see below).

\subsection{Complementary Methods for Parametric Tail-index Estimations based on various Versions of L-moments and the Small Sample Issue}

There have been a number of estimation methods proposed in the literature for recovering the relevant parameters of GEV densities and GPD. As a more efficient alternative to the non-parametric method presented above, the most common practice is to use a direct numerical Maximization of the Log-likelihood of the specified density function. Nevertheless, the Generalized Method of Moments (GMM) by Hansen (1982), that encompasses the Maximum Loglikelihood Estimation (MLE) approach as a special case, overcomes many of the problems identified with the classical method when analyzing large data sets. However, the small sample issue is critical when using these methods (see Delicado and Goria, 2008). Hosking and Wallis (1987) report that the MLE method applied to the GPD is not reliable unless the sample size is larger than 500. They also propose to restrict the values of $\xi$ to the range ] $-.5 ; .5[$ when estimated via MLE. del Castillo and Daoudi (2009) explain in details the anomalous behavior of the Likelihood surface of a GPD and explicit the problem of potential mis-specification for small samples. Facing this issue, Chaouche and Bacro (2006) propose a modification of the Likelihood equation in order to avoid convergence failure. Furthermore, MLE may end with erratic nonsensical solutions when the Shape-parameter of a GEV is close to $-1 / 2$ (Diebolt et al., 2008). The Probability-weighted Moments (PwM) method by Greenwood et al. (1979) is in its turn regarded as the best method for parameter estimation with small samples (Hosking et al., 1985), which is, by definition, the case when studying the extreme events. Diebolt et al. (2008) recommend relying on the Generalized PwM to estimate a GEV density, in particular for large values of the Shape-parameter. Haktanir (1997) proposes in the estimation of the PwM to use the distribution to estimate, in order to compute the non-exceedance probability. This method, called the Self-determined Probability-weighted Moment method (Sd-PwM in short), provides more realistic and more accurate PwM (or Linear Self-determined Moments, LSd-moments hereafter), in relation to the underlying supposed distribution. This last method allows us to detect if a distribution is appropriate for describing the behavior of the sample, and

\footnotetext{
${ }^{12} \mathrm{We}$ also followed the declustering procedure proposed by Coles (2001), only selecting few extrema in each cluster (Block Maxima on Peaks-over-Threshold). These results (not reported herein for space reason) remain once again similar regarding the sign of recovered Tail-indexes in Section 4 below.
} 
also takes the outliers better into account. Nevertheless, the estimation of the distribution parameters involves in this case more complex equations which can only be solved by numerical algorithms (see for example Whalen et al., 2002 and 2004, for a discussion).

Concerning now the estimation of the GPD parameters, Hosking and Wallis (1987) and del Castillo and Hadi (1997) make some recommendations about the estimation method to adopt. When the Shape-parameter is supposed to be in the range $]-.50, .50[$, and the sample size is larger then 500, then the MLE method should be preferred. For small and moderate samples, del Castillo and Hadi (1997) propose a two-step method, called the Elemental Percentile Method (EPM), also inspired by the PwM and the Sd-PwM methods, which seems to perform better for small (inferior to -.40) and large values (superior to .40) of $\xi$. Another answer is given by Vandewalle et al. (2007) concerning the positive tail estimation of a GPD by using the properties of the slowly-varying function that defines Pareto-type distributions. The proposed estimator is based on a robust integrated squared error applied to a mixture model.

In an equivalent manner to the PwM, one can also use the L-moments method for estimating the Tail-index. Defined by Hosking (1986 and 1990) under this name after the seminal work by Sillito (1951 and 1969), the method is based on linear combinations of order statistics. Formally, the population L-moment of order $r \in \mathbb{I}^{*}$ is defined such as:

$$
\lambda_{r}=\sum_{k=1}^{r} p_{r-1, k-1} \beta_{k}
$$

with:

$$
\beta_{k}=k^{-1} E\left(X_{[k: k]}\right)=\int_{\mathfrak{D}} x[F(x)]^{k-1} d F(x),
$$

and where $p_{., .}$are the Legendre polynomials coefficients, which results in:

$$
\lambda_{r}=r^{-1} \sum_{j=0}^{r-1}(-1)^{j}\left(\begin{array}{l}
r-1 \\
j
\end{array}\right) E\left(X_{[r-j: r]}\right) .
$$

Other authors have proposed refinement to the L-moment method, by deleting some extreme points, which leads to methods less subject to outliers and then to biases in estimation. For censored samples, Wang (1990) then introduced the method of Partial Probability-weighted Moments (P-PwM), as an extension of the PwM. In the same manner, Bhattarai (2004) has proposed the Partial L-moments, derived from the L-moments. Moreover, the P-PwM has been generalized by Al-Khodary et al. (2008) with the method of Double Censoring Partial Probability-weighted Moments (DCP-PwM). With the same goal, Wang (1997) also suggest a modified version of L-moments called LH-moments, which (also) are linear functions of the expectations of the highest order statistics (see Hewa et al., 2007, for a practical application to a GEV distribution). Indeed, the L-moments are relatively robust against outliers. However, for the purposes of estimating extreme events (e.g. rainfalls or floods, as in Lee and 
Maeng, 2003), this also might be considered as a weakness rather than an advantage. When one wants to put more emphasis on extreme events, an LH-moment approach allows us to give more weight to the largest ones ${ }^{13}$ as described below.

Formally, the LH-moment of order $r \in \mathbb{N}^{*}$ depends upon one parameter $m \in \mathbb{N}$ and is computed from a conceptual random sample of size $r+m$ as such (with the previous notations):

$$
\lambda_{r}^{H}=r^{-1} \sum_{j=0}^{r-1}(-1)^{j}\left(\begin{array}{l}
r-1 \\
j
\end{array}\right) E\left(X_{[r+m-j: r+m]}\right),
$$

where $m$ denotes the number of lower observations that will be omitted in the computation.

We can also quote a symmetric version of the LH-moments, given by the LL-moments of order $r \in \mathbb{N}^{*}$ which have been proposed by Bayazit and Önöz (2002). They are defined as such (with the previous notations):

$$
\lambda_{r}^{L}=r^{-1} \sum_{j=0}^{r-1}(-1)^{j}\left(\begin{array}{l}
r-1 \\
j
\end{array}\right) E\left(X_{[r-j: r+m]}\right) .
$$

This definition gives more weight to the smaller observations, in order to better fit the lower tail.

Finally, some authors recently pushed further the two last approaches for being even less sensitive to outliers and proposed the Trimmed L-moments (TLmoments in short), first published by Elamir and Seheult (2003). Basically, the idea is also to focus on a part only of the extreme sequence but with another rationale: some extremes should be considered as too extreme, and others as out of the scope of studies of extremes. The general TL-moment of order $r \in \mathbb{N}^{*}$ is defined by two parameters, $s$ and $t$, and by a conceptual random sample of size $r+s+t$, as follows ${ }^{14}$ (with the previous notations):

$$
\lambda_{r}^{(s, t)}=r^{-1} \sum_{j=0}^{r-1}(-1)^{j}\left(\begin{array}{l}
r-1 \\
j
\end{array}\right) E\left(X_{[r+s-j: r+s+t]}\right) .
$$

In this case, the $s$ lowest and the $t$ largest observations are just not considered. The computation of the TL-moment estimator allows us to assign zero

\footnotetext{
${ }^{13}$ However, one notes here that deleting some (false contamining) extrema from the sample under studies in the LH-computation is an idea close to the one aiming to restrict the sample in terms of quantiles of the variable in the (simple) L-moment computation - see below the discussion about the part of extrema - the block maxima length or the threshold for peak selection - to consider when fitting an Extreme Value distribution.

${ }^{14}$ Elamir and Seheult (2003) explicitly present the four first TL(1,1)-moments for a Normal distribution with parameters $(\mu, \sigma): \lambda_{1}^{(1,1)}=\mu, \lambda_{2}^{(1,1)}=.297 \sigma, \lambda_{3}^{(1,1)}=0$ and $\lambda_{4}^{(1,1)}=.018 \sigma$; for a Logistic distribution with parameters $(\mu, \sigma): \lambda_{1}^{(1,1)}=\mu, \lambda_{2}^{(1,1)}=.5 \sigma, \lambda_{3}^{(1,1)}=0$ and $\lambda_{4}^{(1,1)}=.041 \sigma$; for a Cauchy distribution with parameters $(\mu, \sigma): \lambda_{1}^{(1,1)}=\mu, \lambda_{2}^{(1,1)}=.698 \sigma$, $\lambda_{3}^{(1,1)}=0$ and $\lambda_{4}^{(1,1)}=.239 \sigma$; and for an Exponential distribution with parameter $\alpha: \lambda_{1}^{(1,1)}=$ $5 \alpha / 6, \lambda_{2}^{(1,1)}=\alpha / 4, \lambda_{3}^{(1,1)}=2 / 9$ and $\lambda_{4}^{(1,1)}=\alpha / 48$.
} 
weights to some extreme (smallest and highest) observations within the sample. The resulting Trimmed L-moment has the same ease of computation as the Lmoment, but will be less affected by one-shot events, such as an abnormal day in the history of market volatilities ${ }^{15}$. Moreover, TL-moments exist whatever the distribution, and then present a wider range of uses, as for example the measure of symmetry (e.g. Thomas, 2009). We can underline that the TL-moments approach is a more general framework which encompasses the L-moments, the LL-moments and the LH-moments. Indeed, every L-moment $\lambda_{r}$ can be seen as a TL-moment $\lambda_{r}^{(0,0)}$. In the same way, we have $\lambda_{r}^{H}=\lambda_{r}^{(s, 0)}$ and $\lambda_{r}^{L}=\lambda_{r}^{(0, t)}$, for some $(r, s, t) \in\left(\mathbb{N}^{*} \times \mathbb{N}^{2}\right)$.

The application of TL-moments for a practical estimation of extreme distributions requires first computing the analytical link between their values and the characteristic parameters of the densities, which means choosing the number of observations to be trimmed from the sample. Since we are interested in specifying distributions with only one infinite tail, we also present computations with the removal of only the highest value from each sample, as in Hosking (2007).

In finance and more specifically in the case of volatility outliers, it is unclear whether it is desirable to actually neutralize the most extreme observations since the nature of an outlier is difficult to perceive. On the one hand, they might be thought to represent one-of-a-kind events, that will not happen again in the stock's (or indice's) life, and we should then trim them to avoid misleading the estimation of the true underlying (future) density. On the other hand, these types of contingencies have to be provided for a risk assessment and should thus be included - and even emphasized - in computations, whatever their likelihood. The latter argument should lead to the use of L-moments, whilst the former advocates for TL-moments.

Finally, we can also quote another use of TL-moments proposed recently by Darolles et al. (2009). They introduce a new fund performance measure, which is a ratio between modified versions of TL-moments of order 1 and 2, for different shrinkage values. They define Trimmed L-moments from a conceptual sample of odd size, with a symmetry property with respect to the center of the conceptual center. The version of their modified Sharpe ratio, adapted to the TL-moments, is thus supposed to be robust to outliers. More precisely, a battery of $(k, n)$-trimmed L-performances can be defined, for every $n \in \mathbb{N}$ and $0 \leq k \leq n-1$, such as:

$$
\begin{aligned}
L_{k, n}= & \tilde{\lambda}_{1,0, n} / \tilde{\lambda}_{2, k, n} \\
= & E\left(X_{[n+1: 2 n+1]}\right) /\left[E \left(X_{[2 n-k+1: 2 n+1]}\right.\right. \\
& \left.\left.\quad-X_{[k+1: 2 n+1]}\right)\right] .
\end{aligned}
$$

We observe that, for every $0<u<1$, when $k \rightarrow+\infty, n \rightarrow+\infty$ and $k /(2 n+$

\footnotetext{
${ }^{15}$ As explained in Darolles et al. (2009), when considering sample Trimmed L-moments, it is preferable to choose values for $s$ and $t$ much smaller than the size of the population in order to better capture extreme behaviors.
} 
1) $\rightarrow u$, we have:

$$
\lim _{(k, n) \rightarrow \infty} L_{k, n}=\frac{Q_{X}(1 / 2)}{\left[Q_{X}(1-u)-Q_{X}(u)\right]},
$$

where $Q_{X}($.$) is the Quantile Function of the random variable X$.

The denominator of this adapted Sharpe ratio in a L-moment framework is thus a measure of the dispersion of the returns, which can be computed using several quantiles, corresponding to various levels of Value-at-Risk.

Another route for mitigating the effect of potential outliers is proposed by Mudholkar and Hutson (1998), who refine the L-moment also by relying on the Quantile Function of order statistics, replacing the mean by the so-called "quick estimate" of the location parameter of the order statistics. Thus, the LQmoments of order $r \in \mathbb{N}^{*}$ are defined, as before, from the Legendre polynomials, such as:

$$
\lambda_{r}^{Q}=\sum_{k=0}^{r-1} p_{k, r} \tau\left(X_{[r-k: r]}\right),
$$

where $p_{., .}$are the Legendre polynomials coefficients, and $\tau\left(X_{[r-k: r]}\right)$ is a "quick" estimate of the location parameter of the order statistics.

As for TL-moments, LQ-moments always exist since they do not require any moment condition for their existence. As an example, Mudholkar and Hutson (1998) propose location estimators based on one point (the median) and three points (the trimean and Gastwirth) for practical applications of the first three LQ-moments. When using the median of the order statistics, as estimated with the Inverse Beta distribution, the quick estimator is equal to:

$$
\tau\left(X_{[r-k: r]}\right)=Q_{X}\left[B_{X[r-k: r]}(1 / 2)\right],
$$

with $Q_{X}($.$) the Quantile Function and B_{X[r-k: r]}($.$) the Inverse Beta Function.$ Shabri and Jemain (2007) also show, with location estimators based on five points, the superiority of the LQ-moment method over the L-moment one.

Concerning now the estimations, the characteristic parameters of a GEV distribution can analytically be retrieved from the expressions of the Conventionalmoments (Sankarasubramanian and Srinivasan, 1999), the L-moments (Hosking, 1990), the LH-moments (Wang, 1997), the LL-moments (Bayazit and Önöz, 2002), the TL-moments and the LQ-moments (Mudholkar and Hutson, 1998). More precisely, the Shape-parameter will be deduced from the skewness coefficient values (see the Appendix for details). We present in Table 1-a the expressions of the various L-moments depending upon the Shape-parameter of the corresponding matching GEV density, while proofs are left in the Appendix.

- Please, insert Table 1-a somewhere here -

Finally, when it comes to the estimation of population moments, another variation of the Probability-weighted Moments by Greenwood et al. (1979) can be found in Haktanir (1997). He proposes a method called Self-determined 
Probability-weighted Moments (Sd-PwM) as an extension and an improvement of the original $\mathrm{PwM}$ method for parameter estimation. The underlying idea is here to enhance the accuracy (or to highlight the inadequacy) of the PwM by more fully utilizing the mathematical properties of the underlying probability distribution. Indeed, the PwM estimators are obtained only by using the sample order statistics through the non-exceedance probabilities, whatever the distribution to estimate. Thus the parameters of the studied distribution are defined as the values that make the PwM estimators equal to the theoretical PwM of the given distribution. On the contrary, the method of Sd-PwM assumes that the non-exceedance probabilities of the observations can be computed with the $\mathrm{Cu}$ mulative Distribution Function of the assumed distribution. Then, the Sd-PwM are taking into account the supposed distribution. But we can underline that, compared to the previous methods, it is more demanding in terms of mathematical solving. Indeed, finding the parameters of the studied distributions require the simultaneous solving of two non-linear equations (see Tables 1-b and 2-b, and the Appendix for details for the GEV density and the GPD estimations with Linear Self-determined Moments).

- Please, insert Table 1-b somewhere here -

del Castillo and Hadi (1997) also present a similar method to the Sd-PwM one. They propose first to recover quick estimates of the parameters of the distribution and then to use them to define more precisely the PwM estimators. We will use it hereafter as a complement for all other methods, using first the ML parameter estimates as initial starting values in the search for global solutions.

As in the case of the GEV density, the GPD can also be estimated by matching the moments. An estimation of the estimated Shape-parameter of the GPD (denoted $\hat{\xi}$ ) can be retrieved from the C-moments (Hosking and Wallis, 1987), the L-moments (Hosking, 1990; Abdul-Moniem and Selim, 2009), the LHmoments, the LL-moments (Hosking, 2007), the TL-moments (e.g. Elamir and Seheult, 2003; Abdul-Moniem and Selim, 2009), and the LQ-moments (Mudholkar and Hutson, 1998). We present in Table 2-a the expressions of the various L-moments depending upon the Shape-parameter of the corresponding matching GPD, with proofs are reported in the Appendix.

- Please, insert Table 2-a somewhere here -

The Sd-PwM method application for a GPD leads to the following result reported in Table 2-b.

- Please, insert Table 2-b somewhere here -

\section{Extreme Volatility Evidence on the CAC40 Index}

In the next sub-section, we present the estimation of the main measures of volatilities presented above. We use a sample of CAC40 30" quotes, resampled 
at a 30' frequency, from the period 01-1997/05-2009 (1,350,000 original high frequency quotes, leading to 22,500 resampled 30 ' prices, providing 2,500 or so daily volatilities, and from 60 to 250 extrema in the following - depending on the selected quantile). This choice of sampling frequency was guided here by the trade-off between accuracy, theoretically optimized with the highest possible frequency, and the microstructure noise (e.g., Crack and Ledoit, 1996, Oomen, 2005 , and Bandi and Russel, 2006) that is present for very high frequency data ${ }^{16}$. The following sub-section is dedicated to the presentation of the main volatility measure characteristics, whilst the next one presents some results regarding the distributional properties of the extreme of volatility estimates.

\subsection{Time-structure, Correlation and Distribution of Volatil- ity Estimates}

The set of Figures 1 represents the time-series, Probability-to-Probability Plots, Extreme Value densities and Extremal Indexes, of the various daily estimates considered - namely Realized Volatility, Bi-power Variation, Realized Jump Component, Simple Volatility, EMA Volatility, Parkinson (1980), Garman and Klass (1980), Rogers and Satchell (1991), Kunitomo (1992), Yang and Zhang (2000) Extreme Value Volatility estimators - of daily volatilities. The set of figures presented concerned the square root of variance estimates, annualized by multiplying it by the square root of the number of trading days per annum, divided by the number of days in its volatility interval (see Hull and White, 1987).

- Please, insert Figures 1 somewhere here -

The peaks of the variance estimates are approximately synchronous, but the general behavior of the series differs, both in the range of variances and persistence phenomenon (see section 3). The Close-to-Close Volatility appears extremely irregular and very noisy, whereas the Realized Volatility is smoother and displays the phenomenon of persistence of variance far more clearly. In other words, the sample standard deviation underestimates the "true "volatility (even in the absence of noise) as noted for example by Cho and Frees (1988). As already mentioned by Bali and Weinbaum (2005), we see that the Parkinson (1980) estimator is closer in behavior to the Realized Volatility, whereas the additional terms in the various advanced Extreme Value estimators actually seem to make the estimator less regular and in particular produces more very-low volatilities, not unlike the basic Close-to-Close squared return. As crucially emphasized by Alizadeh et al. (2002), range-based estimators have many attractive

\footnotetext{
${ }^{16}$ However, first preliminary tests were based on direct 30" quotes, then ran another time on a 5' aggregation scheme, whilst Maillet et al. (2010) used a 20' sampling rate, with no resulting change in the overall qualitative following results. Additional measures - not reported here for space reason but available on demand - have been also computed for 60' and 120' sampling rates, and lead once again to the same conclusion.
} 
properties over either low frequency estimators or even, for some authors, highfrequency based volatility estimators (Aït-Sahalia et al., 2005; Martens and van Dijk, 2007).

Table 3 presents the main descriptive statistics of the empirical (Log-)volatilities. The asymmetry coefficient is mostly positive (with the exception of RogerSatchell and Simple Volatilities, both exhibiting many very small values); the mass of probability in the right side of the distribution appear slightly larger than on the left side. The kurtosis differs across measures, with once again the Simple Volatility and Rogers-Satchell one appearing heavy-tailed due to the existence of many observations close or equal to zero (a consequence of using these estimators on a daily basis). However, the global statement obtained with the various Probability-to-Probability Plots show some adequacy with the LogNormal (except for the Rogers-Satchell and Simple Volatility which appear to be very different). For very large volatilities, the zoom on extremes seems to confirm that the empirical estimates of the tails are below the Normal ones.

The Extremal Index estimations indicate however the presence of an important clustering effect.

- Please, insert Table 3 somewhere here -

Overall, as already seen in Figure 1-a, estimators using intra-day data are less volatile (more accurate) than the classical estimator.

Many articles have been dedicated to distribution of financial returns (see for instance McDonald, 1994), but comparatively few on the distribution of empirical volatilities (see, for instance, Andersen et al., 2001-a and 2001-b or Thomakos and Wang, 2003). It is now generally admitted since the seminal papers by Cizeau et al. (1997), Liu et al. (1999) and Andersen et al. (2001-a and 2001-b) that the Log-volatility is approximately Gaussian for a daily integratedhorizon. This hypothesis is nevertheless challenged both for ultra-high and low frequencies. On the one hand, the unconditional ultra high-frequency return density being leptokurtic, due to the presence of fat tails but also to a peak around zero-return (no-event) in calendar-time; the density of the volatility at these frequencies should be then degenerated with a high level of probability around zero (zero-return, zero-volatility). On the other hand, unconditional low frequency return density is by contrast approximately almost Gaussian, and the density of the volatility at a low frequency should then be close to a Chi-squared one. As underlined by Forsberg and Bollerslev (2002), this timeaggregation effect should be a characteristic feature of volatility modelling. An unrelated problem is the amplitude of some extreme events, specifically the 1929 and 1987 crises which defy most attempts to assign a probability to such huge variations. Miccichè et al. (2002) show that the right tail of a log-normal density underestimates the probability of the most extreme realizations of the volatility, which are clearly "outliers" for Johansen and Sornette (2001). For Forsberg and Bollerslev (2002), the Normal Inverse Gaussian is a reasonable distribution for the variance (see Barndorff-Nielsen, 1997) - accounting for main induced scale-relation parameters - in the Mixing Distribution Hypothesis of 
Clark (1973). Bontemps and Meddahi (2005) recently confirm this finding quite intensively.

In our sample, Figure 1-b, as well as the Kolomogorov-Smirnov tests we ran (see Table 3), seem to indicate that the differences between empirical kernel estimates and estimated parametric Log-normality are not huge (except for both the Rogers-Satchell estimate series and the Simple Volatility ones, as indicated by the higher-order moments of these series). However, complementary Goodnessof-Fit tests (not all presented here for space reasons) show that Log-normality should be strictly rejected at traditional confidence levels for most of the Logvolatility estimate series (except for Parkinson, Garman-Klass and Yang-Zhang estimations for which we cannot reject the Gaussian hypothesis), since the large number of considered datapoints in our application and the related accumulation of small differences (especially in the tails) cannot be neglected. Neither the Simple Log-volatility nor the Realized Jump Component appear, indeed, to be Normal. This is observable in Figures 1-b and 1-c, which represent the Probability-to-Probability Plot as well as a focus on the extreme volatilities for Gaussian and Log-normal estimations. Similar tests, made not on the volatilities themselves but on the devolatilized Log-volatility series (i.e. the daily Log-volatility divided by its rolling standard deviation or $\operatorname{AR}(1)-\operatorname{GARCH}(1,1)$ log-residuals) would also lead to a rejection of the Gaussian paradigm for all measures. This confirms our instinctive belief of facing an almost approximative normal but not truly Gaussian phenomenon. Regarding now redundancies and common information in the various estimations, the following Table 4 corresponds to the correlation matrices of risk log-estimations.

- Please, insert Table 4 somewhere here -

The Parkinson Volatility is very close to the Realized Volatility and thus can be used as a proxy when all the data is not available intra-daily. Another intraday measure (namely Garman-Klass) is also highly correlated with these two, in contrast with Rogers-Satchell and Yang-Zhang estimators. Lastly the Closeto-Close squared return evolution is relatively poorly correlated with all other measures, even when smoothed with an EMA. These results are confirmed by the analysis of the relative rankings of the observed market sessions according to their volatility (Spearman correlation between measures). The main component of the Realized Volatility appears to be the Bi-power Variation with the Jump Component being overall with a weaker correlation. The empirical Jump Component appears correlated with the Bi-power Variation, making here, surprisingly, the raw decomposition somehow redundant (see Corsi et al., 2009).

\subsection{Extreme Volatilities and Tail-index Estimates}

The Goodness-of-Fit tests presented in the previous section use the information content in the whole range of observations. A rejection can thus be caused by differences in the left hand tail of the data or in the mode and, likewise, small local differences in the curve can be diluted in the whole sample. However, 
a diagnosis of market volatility is relevant in turbulent periods and a certain inaccuracy in the low-risk periods can be tolerated, provided the estimator performs best otherwise, i.e. when risks are important. The aim of the following sub-section is to more focus on extreme events and high watermarks of market risks.

\subsubsection{A Non-parametric Shape-parameter Estimation}

Focusing now on the right part of the density of the volatilities where the extreme lie, we start by computing the Generalized Hill Shape-parameter estimates against the number of observations taken in the calculus as represented in Figure 2.

- Please, insert Figure 2 somewhere here -

This first eye-ball analysis of the Shape-parameters (or inverse of Tail-indexes) corresponding to the various volatility indicators indicates that all they appear to converge and stabilize to a negative value (between -.05 and -.35) as soon as the estimation includes at least the $5 \%$ upper volatilities ${ }^{17}$. However, though these non-parametric techniques are widely used, they may remain inaccurate, relying ultimately on a somewhat subjective graphic analysis for making the distinction between the different types of Extreme Value distributions. It is thus advised to complement these non-parametric estimates with standard parametric estimates from the fit of asymptotic Extreme Value distributions.

\subsubsection{Parametric GEV Density and GPD Tail-index Estimations}

We then turn to parametric estimation, first fitting a GEV density and, secondly, a GPD on the data.

As a first parametric check, we estimate a GEV density of daily volatility maxima, considering three arbitrary block lengths (weekly, monthly and quarterly). This density provides in general a good approximation for the distribution of the maxima, as signalled by the Kolmogorov-Smirnov Goodness-of-Fit tests based on the Maximum Likelihood estimated distributions. The test never rejects the hypothesis that the GEV distribution fits the data with the lowest P-value being .05 for the weekly maxima of the Realized Volatility. We also check that the same result is obtained for other frequencies, namely for monthly and quarterly windows.

As for the second parametric check, we fit a GPD and choose to follow Neftçi (2000) and Bekiros and Georgoutsos (2005) for fixing a first cut-off value at a somewhat arbitrary reasonable level for our purpose. We consider the upper decile of distributions for generating the exceedance data, meaning that it is the value under which $90 \%$ of the sample of volatilities lies. However, for the sake of confirmation, we also estimate the GPD Tail-indexes for the various

\footnotetext{
${ }^{17} \mathrm{We}$ have also reproduced the same analysis with the Pickands estimator, leading to the very same general conclusion.
} 
measures using both supplementary thresholds fixed at $5 \%$ and $2.5 \%$, with no resulting change in the qualitative results. In order to confirm if extremes are really extremes (see Gonzalo and Olmo, 2004), we also checked for all cutoffs, all volatility estimators and all methods of estimations whether the GPDs still provide good approximations for the distributions of the maxima, applying a Kolmogorov-Smirnov Goodness-of-Fit test on the resulting daily volatility Maximum Likelihood estimated distributions. The Kolmogorov-Smirnov test never rejects the hypothesis that the GPD fits the data, with the lowest P-value being .11 for the 2.5\%-threshold peaks of the Yang-Zhang daily volatility series.

Once the Shape-parameter is estimated through the ML method, we can also provide $90 \%$ confidence intervals (5\%-95\%) for the two extreme distributions considered, computed with Log-likelihood functions as in Gilli and Këllezi (2006). For the GEV distribution of the highest Log-volatilities first, the Loglikelihood writes, for $t=[1, \ldots, T]$ :

$$
\mathfrak{L}\left(\sigma_{t} \mid \xi, \alpha, v\right)=\sum_{t=1}^{T} \ln \left[f\left(\sigma_{t} \mid \xi, \alpha, v\right)\right],
$$

where the GEV density is:

$$
\begin{aligned}
f\left(\sigma_{t} \mid \xi, \alpha, v\right)= & \alpha^{-1}\left[1+\xi_{\alpha}\left(\sigma_{t}-v\right)\right]^{-(1+1 / \xi)} \\
& \times \exp \left\{-\left[1+\xi_{\alpha}^{-1}\left(\sigma_{t}-v\right)\right]^{-1 / \xi}\right\},
\end{aligned}
$$

with $\xi_{\alpha}=\alpha / \xi$ and $\xi \in I R^{*}$.

The maximization of the Log-likelihood function $\mathfrak{L}($.$) gives the parameters$ $(\hat{\xi}, \hat{\alpha}, \hat{v})$. Since we are only interested in the confidence interval for $\xi$, one needs first to define the profile Log-likehood function (see Reid, 2000, for details):

$$
\mathfrak{L}^{*}\left(\sigma_{t} \mid \xi\right)=\operatorname{Sup}_{(\alpha, v) \in \mathbb{R}_{+}^{*} \times \mathbb{R}}\left\{\mathfrak{L}\left(\sigma_{t} \mid \xi, \alpha, v\right)\right\} .
$$

Computing the following set:

$$
\Xi=\left\{\xi \in \mathbb{R} \mid \mathfrak{L}\left(\sigma_{t} \mid \hat{\xi}, \hat{\alpha}, \hat{v}\right)-\mathfrak{L}^{*}\left(\sigma_{t} \mid \xi\right) \geq-\frac{1}{2} \chi_{(.1 ; 1)}^{2}\right\}
$$

where $\chi_{(.1 ; 1)}^{2}$ is the $10 \%$ level quantile of the Chi-squared distribution with one degree of freedom, leads to the definition of a $90 \%$ confidence interval for $\hat{\xi}$ using the set $\Xi$, such as:

$$
[\underline{m} ; \bar{m}]=[\min (\Xi) ; \max (\Xi)] \text {. }
$$

The same method is secondly applied for the GPD of the Log-volatilities exceedances. In this case, the density function is given by (with the previous notations):

$$
f\left(\sigma_{t} \mid \xi, \alpha, v\right)=\alpha^{-1}\left[1+\xi_{\alpha}^{-1}\left(\sigma_{t}-v\right)\right]^{-(1+1 / \xi)} .
$$

By considering again the same set $\Xi$ for the Log-likelihood function associated to this density function, we also obtain the confidence interval for the Shape-parameter $\xi$ in the case of a GPD. 


\subsubsection{Complementary Artificial Samples based on Bootstrap and Surrogate Data Techniques}

In order to strengthen our conclusions further, we also generate artificial series based on the properties of the real market data, using first a simple bootstrap method (Efron and Tibshirani, 1994) and, secondly, a constrained randomization surrogate data technique (Schreiber, 1998), applied to the series of highfrequency returns.

For generating the conventional bootstrapped series, we draw with replacement simple returns ${ }^{18}$, then compute the related volatility series. The maxima and peaks are thus close to being zero-correlated by construction; we can then fit with no drawback a GEV density and a GPD on these series, and draw the empirical distribution of Shape-parameters. However, the bootstrap procedure used here is not without criticism, mainly because first the nuisance parameters are far from having no impact on the estimation results, and, secondly, because the volatility series themselves do not respect the IID hypothesis. For instance, in the case of autocorrelations, regime shifts or long memory processes, the BM method for the GEV density estimation may lead to a poor and inaccurate estimation of the true Shape-parameter; similarly, the same effect may exist for the PoT method for the GPD if there is a mis-specification in the relation. More specifically in our context, the volatilities do not conform to the hypotheses required for using the Extreme Value estimators and the related bootstrapping procedure: it is a well known fact that the volatilities of financial assets exhibit a peculiar type of persistence, i.e. short term inertia, potential long memory and/or regime shifts. The extrema tends to occur in clusters and the maximum of daily volatilities over one week might actually hide other volatilities belonging to the tail of the distribution. The same market event might also produce several consecutive periods of high peaks, thus biasing the measure. Moreover, an especially disturbing form of non-stationarity is the possible presence of a structural break within the return series. Indeed, as pointed out by Granger and Hyung (2004), the presence of breaks in time-series can lead to spurious conclusions about long memory. It is thus necessary to also control for the presence of breaks in the sample to be tested. Andreou and Ghysels $(2002,2006)$ derive the limit distributions of the traditional CUMSUM statistics for some dynamic volatility processes. Fernandez (2005) compares a wavelet analysis with the Iterative Cumulative Sum of Squares (ICSS), a variant of the CUMSUM test. It is shown in particular that conditional heteroskedasticity substantially inflates

\footnotetext{
${ }^{18}$ For the sake of both confirmations of our algorithms and results, we also systematically complement our simple bootstrap results using other alternative resampling techniques aiming to keep some of the return and volatility dependence structure. For instance, the complementary four tested procedures on ML estimates of the Shape-parameter of a GEV density (simple bootstrap: Efron and Tibshirani, 1994; stationary: Politis and Romano, 1994; circular: Politis and Romano, 1994, and Politis and White, 2004; accelerated: Efron and Tibshirani, 1994, and Gilli and Këllezi, 2006) used on the original return and on volatility series lead to the very same conclusion regarding the sign of Tail-indexes (the results are not reported here for space reason, but are available on demand). For a discussion about the different methods of block-bootstrap, see Lahiri (1999).
} 
the number of breakpoints detected by both analyses, and thus has also to be controlled.

To get an idea of the amplitude of the non-IID bias and its impact on Tailindex estimation, we complementary use a surrogate data method, explicitly keeping the time-patterns for the returns along a process of constrained randomization, which is basically based on a re-shuffling of the original return data. This procedure thus allows us to generate new artificial samples reproducing some of the main time-series characteristics of the original one. In the case of volatilities, the series are known to exhibit short-term autocorrelation, regime shifts and possibly long memory features.

For the implementation of this technique in our context, we first randomly reorder, several times, the original series of returns, in order to delete all potential time-structure dependences, while keeping intact the unconditional distribution of the series. Secondly, on the new disorganized artificial series, we implement random pairwise permutations until the characteristics of the new series are similar to the original one. More precisely, we apply three tests in order to compare their characteristics to the ones of the original series: a short-term autoregressivity test (see Ljung and Box, 1978, and McLeod and Li, 1983), a long memory one (DFA test, see Kantelhardt et al., 2001) and one for breaks (Inclan and Tiao, 1994). Concerning the short memory characteristics, we focus on the first autocorrelation coefficient of returns and squared returns. For the long memory test, we use as a benchmark parameter the so-called scaling exponent obtained by the Detrended Fluctuation Analysis (DFA ${ }^{19}$, which allows us to detect long-range correlations in time-series with non-linearities. It is based on the computation of a scaling exponent by the mean of a modified root mean square analysis, using local averages to remove the effects of trends and nonstationarities. About the existence of breaks, we call in the test proposed by Inclan and Tiao (1994). Finally, the final surrogate series dataset consists only of series which share the same first autocorrelation coefficients for returns and squared returns $(+/-10 \%)$, the same scaling exponent $(+/-10 \%)$ and the same number of detected breaks.

In the following, we thus have at our disposal for Shape-parameter estimations and inferences, the real market series (22,500 30' quotes), 500 blockbootstrapped artificial series (sharing some unconditional properties of the original ones) and 500 Surrogate series (having the same original density and also similar properties concerning the short-term auto-correlations, long memory and number of significant shifts) of same length (so in total 1,001 series of 22,500 observations).

The following Tables 5 and 6 give the overall aggregated results concerning the estimations of the Shape-parameters related to GEV densities and GPD, for several block frequencies (weekly, monthly and quarterly) and thresholds $(2.5 \%$,

\footnotetext{
${ }^{19}$ In order to confirm the result obtained with such surrogate series, we also generate another set of artificial series, for which the memory parameter is recovered with the local Whittle (1953) estimation method (see Künsch, 1986, and Robinson, 1995) instead of the DFA. It corresponds to a semi-parameric version of the parametric test of Whittle (1953). The same general conclusion applies with these series (results available on demand).
} 
$5 \%$ and $10 \%$ ), using various methods of estimations (MLE and TL-moments) ${ }^{20}$, for the three types of samples considered (original series, bootstrapped and surrogate datas).

- Please, insert Tables 5-a, 5-b, 6-a and 6-b somewhere here -

More precisely, the Table 5-a (Table 5-b) gives the Shape-parameter estimations, their related 90\% confidence intervals and P-statistics of KolmogorovSmirnov Goodness-of-Fit tests (by lines), using the Maximum Likelihood Method (respectively the TL-moment Method) for the GEV density for the daily volatility estimators (by columns), according to weekly, monthly and quarterly block lengths, for each of the three samples (on lines) corresponding to the raw, the simple bootstrapped and the surrogate series.

First of all, all Goodness-of-Fit statistics in Table 5-a lead to the conclusion, to a large extent ${ }^{21}$, that the GEV density corresponds well to the data, clearly indicating that if there is a mis-specification, it should be considered as mild in our sample. Secondly, none of the estimated Tail-indexes is positive, with Shape-parameters ranging from the highest -.08 (for the Yang-Zang estimate) to the lowest -.46 (Garman-Klass Volatility) for the original series of weekly maxima of daily volatilities. Enlarging the block lengths clearly diminish the Shape-parameter whatever the measure, reducing the time-dependence of maxima and relativizing the jumps in comparison to normal variations (but for larger confidence intervals). No general effect can be emphasized regarding the difference between Shape-parameters obtained with the three samples considered. For some estimators however (the Realized Volatility for instance), bootstrapped and surrogate series exhibit lower Shape-parameters. The $90 \%$ confidence intervals have generally a tendency to shrink from the original, bootstrapped and surrogate ones, and get larger when the block length increases. However, no clear general stylized fact is an evidence here, and the effect of the non-IID sample is once again difficult to interpret. Regarding now the Tailindex signs and the various interval confidences, it is obvious that they are all negative for the original series whatever and for all samples for the smallest block lengths. Moreover, only some parts of the upper confidence intervals for the largest block windows are moderately positive.

Table 5-b, giving the same statistics based on the TL-moments Method, confirm these results. Estimated Shape-parameters are rather similar for each estimator when compared to the Maximum Likelihood estimates. Both methods prove here differences (with some parameters being divided by two or so for instance). We also note that the confidence intervals are larger when using the

\footnotetext{
${ }^{20}$ For space and redundancy reasons since the results lead to the same conclusion, we do not report in the following the tables (available on demand) corresponding to the various methods of (L-) moments, namely Conventional moments, L-moments, LH-moments, LL-moments, LQ-moments and LSd-moments, but only the TL(1,1)-moment ones (see Tables 5-b and 6-b). Overall results regarding all methods are, however, represented in Figure 3.

${ }^{21}$ Except for the Roger-Satchell estimator, all P-statistics are far from the traditional probability thresholds.
} 
TL-moments, indicating either a better accuracy to the sub-sample characteristics or a general greater instability, without unfortunately being able, with no extra information, to conclude here.

Table 6-a (results for a GPD using Maximum Likelihood) and Table 6-b (results for a GPD using TL-moments) provide the same global conclusion. Finally, results (not reported here for space reason) based on other versions of the method of moments (namely Conventional-moments, L-moments, LHmoments, LQ-moments and LSd-moments) strengthen this result. The chosen estimation method has generally a fine impact on the value of recovered Shapeparameters; but in our case, all methods converge to the same unique result: most of Tail-indexes are negative and the inference, if not perfect for the longest block window, leads to the conclusion that none of the underlying distributions can be considered as fat-tailed.

Finally, Figure 3 below summarizes the general overall result based on the aggregation of individual results corresponding to all methods used for recovering the Shape-parameter of the extreme volatilities. It represents recovered Shape-parameters related to GEV densities and GPD, for several block frequencies (weekly, monthly and quarterly) or thresholds $(2.5 \%, 5 \%$ and $10 \%)$, using the various methods of estimations (e.g. MLE, C-moments, L-moments, TLmoments, LSd-moments and LQ-moments) for the three types of samples considered (i.e. original series, bootstrapped and surrogate datas) for the ten volatility estimators studied (namely, Realized, Realized Bi-power, Realized Jump Component, Parkinson, Kunitomo, Rogers-Satchell, Garman-Klass, Yang-Zang, EMA and Simple Volatilities), as well as the 5\%-95\% (minimum-maximum) ranges corresponding to every estimation, and all Kolmogorov-Smirnov P-statistics related to the adequation of the related estimated density with the data. Each density presented here corresponds thus to 1,080 estimations of mean (and 5\%minimum / 95\%-maximum) Shape-parameters of, first, one original data of $1,350,000$ high frequency quotes, resampled into 22,500 30' prices, and of, secondly, 1,000 series of 22,500 artificial (Bootstraped and Surrogate) prices of same length as the original.

- Please, insert Figure 3 somewhere here -

This Figure clearly indicates that the Shape-parameter is generally negative (with a mean equal to -.16 and $98.11 \%$ of estimates below .00), whatever the global method of estimation. All 5\%-minimum estimates are indeed negative, and, even if not perfect, the inference for the 95\%-maximum estimations leads to a reasonable percentage of them being slightly positive (with a mean at -.01 and $62.66 \%$ being inferior or equal to .00). Furthermore, most of the Kolmogorov-Sminrov P-statistics reported in this Figure (on the right) are above the $1 \% / 5 \% / 10 \%$ classical significance thresholds (with a mean equal to $58.49 \%$ and with, respectively, $99.99 \% / 100.00 \% / 100.00 \%$ being above these usual significance levels), meaning the fitted Extreme Value densities with estimated parameters are quite adequate. These overall results reinforce our previous conclusions related to Figure 2 (corresponding to non-parametric estimates), and 
Tables 5 and 6 , yielding, most of the time, negative estimations of the Shapeparameter of the extreme volatility distributions.

\section{Conclusion}

The Realized Volatility, despite its known shortcomings, remains a benchmark to which measures of risk should be compared. We have shown here that, amid the low-frequency measures, the instantaneous Parkinson volatility was the one approaching the closest the high-frequency measure. Due to its simplicity, it should thus be the one retained when trying to obtain long-horizon historical estimates, or to complement a series of Realized Volatilities.

Estimations of the whole distribution of the empirical volatilities cannot easily distinguish between the candidate functional forms, and the conclusion may depend upon the sampling frequency, period, horizon, asset and market considered. Given the rationale for estimating these distributions - retrieving possible risk - and the main differences between them - in the tail - it is natural to try instead to use the Extreme Value Theory and concentrate on estimating the asymptotic distribution for the extreme measures of risk. However, some precautions should be taken in regarding the conclusions based on real market estimates for several reasons. First, the impact of nuisance parameters used in the main estimation techniques or robustness tests (such as the Block Maxima length, the threshold values, the block window for the bootstrap...) is not null. Secondly, even if a large quantity of observations is usually available in high-frequency databases, studying the extremes is still however a small sample problem per definition. Thirdly, high frequency financial data suffers from microstructure biases, that are always present by nature, even if extra precautions and optimal sampling frequencies are considered. Lastly, extreme volatilities are known for not conforming to the IID ideal case and thus the unconditional estimation results are essentially linked to the total structural dependence in the data. For all these reasons, we provide estimation results making the parameters vary, as well as employing techniques that are aiming to attenuate both small sample and non-IID effects on estimates. As a cross-product, we mobilize variants of L-moments and generalize some previous results regarding the relation between the various alternative L-moments and the characteristic parameters of both main Extreme Value densities, namely the Generalized Extreme Value density and the Generalized Pareto Distribution.

Altogether with the Non-parametric Generalized Hill estimates, the actual and overall estimations for both the Generalized Extreme Value density and the Generalized Pareto Distribution, clearly indicate that heavy-tailed distributions are not needed to fit our finite sample of volatilities, on the asset and period considered $^{22}$. A log-normal process, as in the traditional stochastic volatility model, seems able to reproduce the extreme empirical volatilities observed in

\footnotetext{
${ }^{22}$ Several extra tests (available on demand) show that neither some specific recent extreme events (i.e. the latest financial crises), nor the global level of market turbulence, have a great impact on this general result.
} 
our twelve-year ultra high-frequency sample. As expected, the Realized Jump Component of the Realized Volatility is mainly responsible for the shape of the distribution of the measure, since it contains (almost) all the peaks.

As previously shown in Maillet et al. (2010), a final reality check, reported in Table 7, shows inadequacies of some of the simplest measures of risk, since they either greatly underestimate or largely overestimate the Return-times for the peak events observed in the sample. The use of the Tail-index estimates, relative to the Realized Volatilities, however, produces some more reasonablelooking values for the Return-times of the high watermarks of volatility.

- Please, insert Table 7 somewhere here -

In the vein of Gonzalez-Rivera et al. (2004), a natural expansion of this article will be to extend the studies to a basket of individual stocks in a true Reality Check framework (White, 2000), to preserve our results from datasnooping (see also Hansen, 2005; Romano and Wolf, 2005; Hsu and Kuan, 2005; Qi and $\mathrm{Wu}, 2006$; Hsu and Hsu, 2007), in which stocks, samples, periods, estimators, nuisance parameters and estimation methods can be bootstrapped, aiming to the construction of a formal test. Focusing this time on recent high frequency estimators of Realized Volatility ( $C f$. Zhang et al., 2005; Aït-Sahalia et al., 2005 and 2009; Zhang, 2006; Vuorenmaa, 2008; Barndorff-Nielsen et al., 2008; Andersen et al., 2009-b; Corsi et al., 2009; Mancini, 2009; Saichev et al., 2009 and 2010), and on other conditional low-frequency alternatives (Engle, 2002; Engle and Gallo, 2006; Engle and Rangle, 2008), it would also be of interest to replicate our studies and compare these estimators on a Tail-index basis (see Vuorenmaa, 2008). Another direction would be to consider, as in Bali and Weinbaum (2007) or in Jalal and Rockinger (2008), a conditional approach for the modelled volatility. Indeed, the estimations we made in this paper are using the assumption that the non-IID characteristic of the underlying process does not have a strong impact on our results. In order to upgrade them and to take fully into account the clustering phenomenon, it should then be interesting, even if not perfect, to model the volatility estimators through ARCH or GARCH processes.

A final practical financial application of these results will be to plug the appropriate estimates and distributions of extreme volatilities in the Index of Market Shocks (IMS, see Maillet and Michel, 2003 and 2005), in order to recover an accurate universal scale of historical crises, and thus a more precise estimation of the return-times of extreme scenarii in financial markets.

\section{References}

[1] Abdul-Moniem I. and Y. Selim, (2009), "TL-moments and L-moments Estimation for the Generalized Pareto Distribution", Applied Mathematical Sciences 3(1), 43-52. 
[2] Aït-Sahalia Y., P. Mykland and L. Zhang, (2005), "How Often to sample a Continuous-time Process in the Presence of Market Microstructure Noise", Review of Financial Studies 18(2), 351-416.

[3] Adler R., R. Feldman and M. Taqqu, (1998), A Practical Guide to Heavy Tails: Statistical Techniques and Applications, Adler-Feldman-Taqqu Eds, Birkhäuser, Boston, 552 pages.

[4] Aït-Sahalia Y., P. Mykland and L. Zhang, (2009), "Ultra High-frequency Volatility Estimation with Dependent Microstructure Noise", Working Paper, University of Princeton, 35 pages.

[5] Alizadeh S., M. Brandt and F. Diebold, (2002), "Range-based Estimation of Stochastic Volatility Models", Journal of Finance 57(3), 1047-1091.

[6] Al-Khodary E., A. Hassan and S. Allam, (2008), "Double Censoring Partial Probability-weighted Moments Estimation of the Generalized Exponential Distribution", InterStat 1, 24 pages.

[7] Andersen T. and T. Bollerslev, (1997-a), "Heterogeneous Information Arrivals and Return Volatility Dynamics: Uncovering the Long-run in Highfrequency Returns", Journal of Finance 52(3), 975-1005.

[8] Andersen T. and T. Bollerslev (1997-b), "intra-day Periodicity and Volatility Persistence in Financial Markets", Journal of Empirical Finance 4(2-3), 115-158.

[9] Andersen T. and T. Bollerslev, (1998-a), "Answering the Skeptics: Yes, Standard Volatility Models do provide Accurate Forecasts", International Economic Review 39(4), 885-905.

[10] Andersen T. and T. Bollerslev, (1998-b), "Deutsche Mark-dollar Volatility: intra-day Activity Patterns, Macroeconomic Announcements, and Longer Run Dependencies", Journal of Finance 53(1), 219-253.

[11] Andersen T., T. Bollerslev and F. Diebold, (2009-a), "Parametric and Non-parametric Volatility Measurement", Chapter 2 in Handbook of Financial Econometrics - Tools and Applications, Aït-Sahalia-Hansen Eds, Elsevier, North-Holland, 67-138.

[12] Andersen T., T. Bollerslev, F. Diebold and H. Ebens, (2001-a), "The Distribution of Realized Stock Return Volatility", Journal of Financial Economics 61(1), 43-76.

[13] Andersen T., T. Bollerslev, F. Diebold and P. Labys, (2001-b), "The Distribution of Realized Exchange Rate Volatility", Journal of the American Statistical Association 96(453), 42-55.

[14] Andersen T., T. Bollerslev, F. Diebold and P. Labys, (2003), "Modelling and Forecasting Realized Volatility", Econometrica 71(2), 579-625. 
[15] Andersen T., D. Dobrev and E. Schaumburg, (2009-b), "Jump-robust Volatility Estimation using Nearest Neighbor Truncation", CREATES Research Paper 2009-52, AARHUS University, 38 pages.

[16] Andreou E. and E. Ghysels, (2002), "Detecting Multiple Breaks in Financial Market Volatility Dynamics", Journal of Applied Econometrics 17(5), 579-600.

[17] Andreou E. and E. Ghysels, (2006), "Monitoring Disruptions in Financial Markets", Journal of Econometrics 135(1-2), 77-124.

[18] Areal N. and S. Taylor, (2002), "The Realized Volatility of FTSE-100 Futures Prices", Journal of Futures Markets 22(7), 627-648.

[19] Bai X., J. Russell and G. Tiao, (2001), "Beyond Merton's Utopia (I): Effects of Non-normality and Dependence on the Precision of Variance Estimates using High-frequency Financial Data", Unpublished Manuscript, Graduate School of Business, University of Chicago, 48 pages.

[20] Bali T., (2003-a), "An Extreme Value Approach to estimating Volatility and Value-at-Risk", Journal of Business 76(1), 83-108.

[21] Bali T., (2003-b), "The Generalized Extreme Value Distribution", Economics Letters 79(3), 423-427.

[22] Bali T. and D. Weinbaum, (2005), "A Comparative Study of Alternative Extreme Value Volatility Estimators", Journal of Futures Markets 25(9), 873-892.

[23] Bali T. and D. Weinbaum, (2007), "A Conditional Extreme Value Volatility Estimator based on High-frequency Returns", Journal of Economic Dynamics \& Control 31(2), 361-397.

[24] Balkema A. and L. de Haan, (1974), "Residual Life Time at Great Age", Annals of Probability 2(5), 792-804.

[25] Ball C. and W. Torous, (1983), "A Simplified Jump Process for Common Stock Returns", Journal of Financial and Quantitative Analysis 18(1), $53-65$.

[26] Bandi F. and J. Russell, (2006), "Separating Microstructure Noise from Volatility", Journal of Financial Economics 79(3), 655-692.

[27] Barndorff-Nielsen O., (1997), "Normal Inverse Gaussian Distributions and Stochastic Volatility Modelling", Scandinavian Journal of Statistics 24(1), 1-13.

[28] Barndorff-Nielsen O. and N. Shephard, (2002-a), "Estimating Quadratic Variation using Realized Variance", Journal of Applied Econometrics $17(5), 457-477$. 
[29] Barndorff-Nielsen O. and N. Shephard, (2002-b), "Econometric Analysis of Realized Volatility and its Use in estimating Stochastic Volatility Models", Journal of the Royal Statistical Society: Series B 64(2), 253-280.

[30] Barndorff-Nielsen O. and N. Shephard, (2003), "How Accurate is the Asymptotic Approximation to the Distribution of Realized Volatility?", in Identification and Inference for Econometric Models, Andrews-PowellRuud-Stock Eds, Econometric Society Monograph Series, Cambridge University Press, 306-331.

[31] Barndorff-Nielsen O. and N. Shephard, (2004), "Power and Bi-power Variation with Stochastic Volatility and Jumps", Journal of Financial Econometrics 2(1), 1-37.

[32] Barndorff-Nielsen O., P. Hansen, A. Lunde and N. Shephard, (2008), "Designing Realized Kernels to measure the Ex-post Variation of Equity Prices in the Presence of Noise", Econometrica 76(6), 1481-1536.

[33] Bayazit M. and B. Önöz, (2002), "LL-moments for Estimating Low-flow Quantiles", Hydrological Sciences 47(5), 707-720.

[34] Beirlant J., Y. Goegebeur, J. Segers and J. Teugels, (2004), Statistics of Extremes: Theory and Applications, Wiley Series in Probability and Statistics, John Wiley \& Sons, Chippenham, 522 pages.

[35] Beirlant J., P. Vynckier and J.-L. Teugels, (1996), "Excess Functions and Estimation of the Extreme Value Index", Bernoulli 2(4), 293-318.

[36] Bekiros S. and D. Georgoutsos, (2005), "Estimation of Value-at-Risk by Extreme Value and Conventional Methods: A Comparative Evaluation of their Predictive Performance", Journal of International Financial Markets, Institutions and Money 15(3), 209-228.

[37] Ben-Zvi A. and B. Azmon, (1997), "Joint Use of L-moment Diagram and Goodness-of-Fit Test: A Case Study of Diverse Series", Journal of Hydrology 198(1-4), 245-259.

[38] Bhattarai K., (2004), "Partial L-moments for the Analysis of Censored Flood Samples", Hydrological Sciences 49(5), 855-868.

[39] Bollen B. and B. Inder, (2002), "Estimating Daily Volatility in Financial Markets utilizing intra-day Data", Journal of Empirical Finance 9(5), 551-562.

[40] Bollen B. and B. Inder, (2003), "A Comparison of Estimators Daily Realized Volatility", Finance Letters 1(1), 29-34.

[41] Bontemps Ch. and N. Meddahi, (2005), "Testing Normality: A GMM Approach", Journal of Econometrics 124(1), 149-186. 
[42] Box G. and D. Cox, (1964), "An Analysis of Transformations", Journal of the Royal Statistical Society: Series B 26(2), 211-264.

[43] Brandt M. and F. Diebold, (2006), "A No-arbitrage Approach to Rangebased Estimation of Return Covariances and Correlations", Journal of Business 79(1), 61-74.

[44] Brunetti C. and P. Lildholdt, (2002), "Return-based and Range-based (Co)Variance Estimation - with an Application to Foreign Exchange Markets", Working Paper Series 127, University of Aarhus, November 2002, 57 pages.

[45] Brownlees Ch. and G. Gallo, (2010), "Comparison of Volatility Measures: A Risk Management Perspective", Journal of Financial Econometrics 8(1), 29-56.

[46] Byström H., (2004), "Managing Extreme Risks in Tranquil and Volatile Markets using Conditional Extreme Value Theory", International Review of Financial Analysis 13(2), 133-152.

[47] Caeiro F. and M. Gomes, (2008), "Minimum-variance Reduced-bias Tailindex and High Quantile Estimation", REVSTAT - Statistical Journal $6(1), 1-20$.

[48] Caers J., J. Beirlant and P. Vynckier, (1998), "Bootstrap Confidence Intervals for Tail- Indices", Computational Statistics \& Data Analysis 26(3), 259-277.

[49] Carrillo S., N. Hernández and L. Seco, (2006-a), "New Families of Distributions fitting L-moments for Modelling Financial Data", RiskLab Technical Paper, 42 pages.

[50] Carrillo S., N. Hernández and L. Seco, (2006-b), "A Theoretical Comparison between Moments and L-moments", RiskLab Technical Paper, 23 pages.

[51] Chaouche A. and J.-N. Bacro, (2006), "Statistical Inference for the Generalized Pareto Distribution: Maximum Likelihood Revisited", Communications in Statistics: Theory and Methods 35(4-6), 785-802.

[52] Christensen K. and M. Podolskij, (2007), "Realized Range-based Estimation of Integrated Variance", Journal of Econometrics 141(2), 323-349.

[53] Cho D. and E. Frees, (1988), "Estimating the Volatility of Discrete Stock Prices", Journal of Finance 43(2), 451-466.

[54] Chu B. and M. Salmon, (2008), "Testing Distributional Assumptions: A L-moment Approach", Unpublished Working Paper, Warwick Business School, September 2008, 45 pages. 
[55] Cizeau P., Y. Liu, M. Meyer, C.-K. Peng and H. Stanley, (1997), "Volatility Distribution in the S\&P500 Stock Index", Physica A 245(3), 441-447.

[56] Clark P., (1973), "A Subordinated Stochastic Process Model with Finite Variance for Speculative Prices", Econometrica 41(1), 135-155.

[57] Coles S., (2001), An Introduction to Statistical Modeling of Extreme Values, Springer Series in Statistics, Springer-Verlag, London, 224 pages.

[58] Cont R., (2001), "Empirical Properties of Asset Returns: Stylized Facts and Statistical Issues", Quantitative Finance 1(2), 223-236.

[59] Corrado Ch. and Th. Miller, (2006), "Estimating Expected Excess Returns using Historical and Option-implied Volatility", Journal of Financial Research 29(1), 95-112.

[60] Corrado Ch. and C. Truong, (2007), "Forecasting Stock Index Volatility: Comparing Implied Volatility and the intra-day High-Low Price Range", Journal of Financial Research 30(2), 201-215.

[61] Corsi F., (2009), "A Simple Approximate Long-memory Model of Realized Volatility", Journal of Financial Econometrics 7(2), 174-196.

[62] Corsi F., D. Pirino and R. Reno, (2009), "Volatility Forecasting: the Jumps do Matter", Global COE Hi-Stat Discussion Paper Series 09-036, Hitotsubashi University, 45 pages.

[63] Corsi F., G. Zumbach, U. Müller and M. Dacorogna, (2001), "Consistent High-precision Volatility from High-frequency Data", Economic Notes 30(2), 183-204.

[64] Crack T. and O. Ledoit, (1996), "Robust Structure without Predictability: The "Compass Rose" Pattern of the Stock Market", Journal of Finance 51(2), 751-762.

[65] Danielsson J. and C. de Vries, (1997), "Tail-index and Quantile Estimation with Very High-frequency Data", Journal of Empirical Finance 4(2), 241257.

[66] Danielsson J. and C. de Vries, (2000), "Value-at-Risk and Extreme Returns", Annales d'Economie et de Statistique 60, 239-270.

[67] Danielsson J., L. de Haan, L. Peng and C. de Vries, (2001), "Using a Bootstrap Method to choose the Sample Fraction in Tail-index Estimation", Journal of Multivariate Analysis 76(2), 226-248.

[68] Darolles S., C. Gouriéroux and J. Jasiak, (2009), "L-performance with an Application to Hedge Funds", Journal of Empirical Finance 16(4), 671-685. 
[69] da Silva A. and B. de Melo Mendes, (2003), "Value-at-Risk and Extreme Returns in Asian Stock Markets", International Journal of Business 8(1), $17-40$.

[70] de Haan L. and A. Ferreira, (2006), Extreme Value Theory: An Introduction, Mikosch-Robinson-Resnick Eds, Springer Series in Operations Research and Financial Engineering, Springer-Verlag, New York, 418 pages.

[71] de Haan L. and S. Resnick, (1980), "A Simple Asymptotic Estimate for the Heavy-tailed Time-series: An Overview of the Theory with Index of a Stable Distribution", Journal of the Royal Statistical Society B 42(1), 83-87.

[72] De Michele C. and G. Salvadori, (2003), "A Generalized Pareto Intensityduration Model of Storm Rainfall exploiting 2-Copulas", Journal of Geophysical Research 108(D2), 40-67.

[73] del Castillo E. and A. Hadi, (1997), "Fitting the Generalized Pareto Distribution to Data", Journal of the American Statistical Association 92(440), 1609-1620.

[74] del Castillo E. and J. Daoudi, (2009), "Estimation of the Generalized Pareto Distribution", Statistics \& Probability Letters 79(5), 684-688.

[75] Delicado P. and M. Goria, (2008), "A Small Sample Comparison of Maximum Likelihood, Moments and L-moments Methods for the Asymmetric Exponential Power Distribution", Computational Statistics 85 Data Analysis 52(3), 1661-1673.

[76] Dekkers A., J. Einmahl and L. de Haan, (1989), "A Moment Estimator for the Index of an Extreme Value Distribution", Annals of Statistics 17(4), 1833-1855.

[77] Diebolt J., A. Guillou, P. Naveau and P. Ribereau, (2008), "Improving Probability-weighted Moment Methods for the Generalized Extreme Value Distribution", REVSTAT - Statistical Journal 6(1), 33-50.

[78] Ding Y., B. Cheng and Z. Jiang, (2008), "A Newly-discovered GPD-GEV Relationship together with comparing their Models of Extreme Precipitation in Summer", Advances in Atmospheric Sciences 25(3), 507-516.

[79] Draisma G., L. de Haan, L. Peng and T. Pereira, (1999), "A Bootstrapbased Method to achieve Optimality in estimating the Extreme-value Index", Extremes 2(4), 367-404.

[80] Drees H., (2003), "Extreme Quantile Estimation for Dependent Data, with Applications to Finance", Bernoulli 9(1), 617-657.

[81] DuMouchel W., (1983), "Estimating the Stable Index $\alpha$ in order to measure Tail Thickness: A Critique", Annals of Statistics 11(4), 1019-1031. 
[82] Efron B. and R. Tibshirani, (1994), An Introduction to the Bootstrap, Chapman \& Hall/CRC, New York, 456 pages.

[83] Elamir E. and A. Seheult, (2003), "Trimmed L-moments", Computational Statistics and Data Analysis 43(3), 299-314.

[84] Embrechts P., C. Klüppelberg and T. Mikosch, (1997), Modelling Extremal Events for Insurance and Finance, Stochastic Modelling and Applied Probability 33, Springer-Verlag, 648 pages.

[85] Engle R., (2002), "New Frontiers for ARCH Models", Journal of Applied Econometrics 17(5), 425-446.

[86] Engle R. and G. Gallo, (2006), "A Multiple Indicators Model for Volatility using Intra-daily Data", Journal of Econometrics 131(1-2), 3-27.

[87] Engle R. and J. Rangel, (2008), "The Spline-GARCH Model for Lowfrequency Volatility and its Global Macroeconomic Causes", Review of Financial Studies 21(3), 1187-1222.

[88] Feller W., (1951), "The Asymptotic Distribution of the Range of Sums of Independent Random Variables", Annals of Mathematical Statistics 22(3), 427-432.

[89] Fernandez V., (2005), "Structural Breakpoints in Volatility in International Markets", IIIS Discussion Paper 76, 33 pages.

[90] Finkenstädt B. and H. Rootzén, (2003), Extreme Values in Finance, Telecommunications, and the Environment, Monographs on Statistics and Applied Probability 99, Finkenstädt-Rootzén Eds, Chapman \& Hall/CRC, 432 pages.

[91] Fisher R. and L. Tippett, (1928), "Limiting Forms of the Frequency Distribution of the Largest or Smallest Member of a Sample", Proceedings of the Cambridge Philosophical Society 24(2), 180-190.

[92] French C., (2008), "The Tail that Wags the Hedge Fund Dog", Proceedings of the JOIM Conference (Boston), 22 pages.

[93] Forsberg L. and T. Bollerslev, (2002), "Bridging the Gap between the Distribution of Realized (ECU) Volatility and ARCH Modelling (of the EURO): The GARCH-NIG Model", Journal of Applied Econometrics $17(5), 535-548$.

[94] Gabaix X. and R. Ibragimov, (2009), "Rank -1/2: A Simple Way to improve the OLS Estimation of Tail Exponents", Harvard Institute of Economic Research Discussion Paper 2106, 39 pages.

[95] Garman M. and M. Klass, (1980), "On the Estimation of Security Price Volatilities from Historical Data", Journal of Business 53(1), 67-78. 
[96] Gettinby G., C. Sinclair, D. Power and R. Brown, (2006), "An Analysis of the Distribution of Extremes in Indices of Share Returns in the US, UK and Japan from 1963 to 2000", International Journal of Finance and Economics 11(2), 97-113.

[97] Gilli M. and E. Këllezi, (2006), “An Application of Extreme Value Theory for Measuring Financial Risk", Computational Economics 27(2), 207-228.

[98] Gomes M. and O. Oliveira, (2001), "The Bootstrap Methodology in Statistics of Extremes - Choice of the Optimal Sample Fraction", Extremes 4(4), 331-358.

[99] Gonzalez-Rivera G., T. Lee and S. Mishra, (2004), "Forecasting Volatility: A Reality Check based on Option Pricing, Utility Function, Value-at-risk, and Predictive Likelihood", International Journal of Forecasting 20(4), 629-645.

[100] Gonzalo J. and J. Olmo, (2004), "Which Extreme Values are Really Extremes?", Journal of Financial Econometrics (2)3, 349-369.

[101] Gouriéroux C. and J. Jasiak, (2008), "Dynamic Quantile Models", Journal of Econometrics 147(1), 198-205.

[102] Granger C. and N. Hyung, (2004), "Occasional Structural Breaks and Long-memory with an Application to the S\&P Absolute Stock Returns", Journal of Empirical Finance 11(3), 399-421.

[103] Greenwood J., J. Landwehr, N. Matalas and J. Wallis, (1979), "Probability-weighted Moments: Definition and Relation to Parameters of Distributions Expressible in Inverse Form", Water Resources Research 15(5), 1049-1054.

[104] Guttman N., J. Hosking and J. Wallis, (1993), "Regional Precipitation Quantile Values for the Continental US computed from L-Moments", Journal of Climate 6(12), 2326-2340.

[105] Haktanir T., (1997), "Self-determined Probability-weighted Moments Method and its Application to Various Distributions", Journal of Hydrology 194(1-4), 180-200.

[106] Hall P., (1990), "Using the Bootstrap to estimate Mean Squared Error and Select Smoothing Parameter in Non-parametric Problems", Journal of Multivariate Analysis 32(2), 177-203.

[107] Hansen P., (1982), "Large Sample Properties of Generalized Method of Moments Estimators", Econometrica 50(4), 1029-1054.

[108] Hansen P., (2005), "A Test for Superior Predictive Ability", Journal of Business and Economic Statistics 23, 365-380. 
[109] Hewa G., Q. Wang, T. McMahon, R. Nathan and M. Peel, (2007), "Generalized Extreme Value Distribution fitted by LH-moments for Low-flow Frequency Analysis", Water Resources Research 43(6), 1029-1038.

[110] Hill B., (1975), "A Simple General Approach to Inference about the Tail of a Distribution", Annals of Statistics 3(5), 1163-1174.

[111] Høg E. and A. Lunde, (2003), "Wavelet Estimation of Integrated Volatility", Working Paper, Aarhus School of Business, Department of Information Science, August 2003, 20 pages.

[112] Hol E. and S. Koopman, (2002), "Stock Index Volatility Forecasting with High Frequency Data", Tinbergen Institute Discussion Paper $N^{\circ}$ 2002068/4, 25 pages.

[113] Hosking, J., (1986), "The Theory of Probability-weighted Moments", Research Report RC12210, IBM Research Division, Yorktown Heights, 15 pages.

[114] Hosking J., (1990), "L-moments: Analysis and Estimation of Distributions using Linear Combinations of Order Statistics", Journal of the Royal Statistical Society: Series B 52(1), 105-124.

[115] Hosking J., (1992), "Moments or L-moments? An Example comparing Two Measures of Distributional Shape", The American Statistician 46(3), 186-189.

[116] Hosking J., (2007), "Some Theory and Practical Uses of Trimmed Lmoments", Journal of Statistical Planning and Inference 137(9), 30243039 .

[117] Hosking J. and J. Wallis, (1987), "Parameter and Quantile Estimation for the Generalized Pareto Distribution", Technometrics 29(3), 339-349.

[118] Hosking J. and J. Wallis, (1997), Regional Frequency Analysis - An Approach based on L-moments, Cambridge University Press, 240 pages.

[119] Hosking J., G. Bonti and D. Siegel, (2000), "Beyond the Log-normal", Risk 13(5), 59-62.

[120] Hosking J., J. Wallis and E. Wood, (1985), "Estimation of the Generalized Extreme Value Distribution by the Method of Probability-weighted Moments", Technometrics 27(3), 251-261.

[121] Hsing T., (1991), "Estimating the Parameters of Rare Events", Stochastic Processes and their Applications 37(1), 117-139.

[122] Hsing T., J. Hüsler and M. Leadbetter, (1988), "On the Exceedance Point Process for a Stationary Sequence", Probability Theory and Related Fields 78(1), 97-112. 
[123] Hsu P.-H. and Y.-C. Hsu, (2006), "A Stepwise SPA Test for Data Snooping and its Application on Fund Performance Evaluation", SSRN Working Paper, 31 pages.

[124] Hsu P.-H. and C.-M. Kuan, (2005), "Reexamining the Profitability of Technical Analysis with Data Snooping Checks", Journal of Financial Econometrics 3(4), 606-628.

[125] Huisman R., K. Koedijk, C. Kool and F. Palm, (2001), "Tail-index Estimates in Small Samples", Journal of Business and Economic Statistics 19(2), 208-216.

[126] Hull J. and A. White, (1987), "The Pricing of Options on Assets with Stochastic Volatilities", Journal of Finance 42(2), 281-300.

[127] Inclan C. and G. Tiao, (1994), "Use of Cumulative Sums of Squares for Retrospective Detection of Changes of Variance", Journal of the American Statistical Association 89(427), 913-923.

[128] Jalal A. and M. Rockinger, (2008), "Predicting Tail-related Risk Measures: The Consequences of using GARCH Filters for non-GARCH Data", Journal of Empirical Finance 15(5), 868-877.

[129] Jenkinson A., (1955), "The Frequency Distribution of the Annual Maximum (or Minimum) Values of Meteorological Elements", Quarterly Journal of the Royal Meteorological Society 81(348), 158-171.

[130] Johansen A. and D. Sornette, (2001), "Large Stock Market Price Drawdowns are Outliers", Risk 4(2), 69-110.

[131] Jondeau E. and M. Rockinger, (2003), "Testing for Differences in the Tails of Stock-Market Returns", Journal of Empirical Finance 10(5), 559-581.

[132] Kantelhardt J., E. Koscielny-Bunde, H. Rego, S. Havlin and A. Bunde, (2001), "Detecting Long-range Correlations with Detrended Fluctuation Analysis", Physica A 295(3-4), 441-454.

[133] Karvanen J., (2006), "Estimation of Quantile Mixtures via L-moments and Trimmed L-moments", Computational Statistics \& Data Analysis 51(2), 947-959.

[134] Katz R., G. Brush and M. Parlange, (2005), "Statistics of Extremes: Modeling Ecological Disturbances", Ecology 86(5), 1124-1134.

[135] Kearns P. and A. Pagan, (1997), "Estimating the Density Tail-index for Financial Time-series", Review of Economics and Statistics 79(2), 171175 .

[136] Kliche D., P. Smith and R. Johnson, (2008), "L-moment Estimators as Applied to Gamma Drop Size Distributions", Journal of Applied Meteorology and Climatology 47(12), 3117-3130. 
[137] Künsch H., (1986), "Statistical Aspects of Self-similar Processes", Proceedings of the First World Congress of the Bernoulli Society (Tashkent) 1, 67-74.

[138] Kunitomo N., (1992), "Improving the Parkinson Method of Estimating Security Price Volatilities", Journal of Business 65(2), 295-302.

[139] Lahiri S., (1999), "Theoretical Comparisons of Block-bootstrap Methods", Annals of Statistics 27(1), 386-404.

[140] LeBaron B., (2009), "Robust Properties of Stock Return Tails", Unpublished Working Paper, 31 pages.

[141] Lee S. and S. Maeng, (2003), "Frequency Analysis of Extreme Rainfall using L-moment", Irrigation and Drainage 52(3), 219-230.

[142] Li K. and D. Weinbaum, (2001), "The Empirical Performance of Alternative Extreme Value Volatility Estimators", Working Paper, New York University, 43 pages.

[143] Liu Y., P. Gopikrishnan, P. Cizeau, M. Mayer, C.-K. Peng and H. Stanley, (1999), "Statistical Properties of the Volatility of Price Fluctuation", Physical Review E 60(2), 1390-1400.

[144] Ljung G. and G. Box, (1978), "On a Measure of a Lack of Fit in Timeseries Models", Biometrika 65(2), 297-303.

[145] Longin F., (1996) "The Asymptotic Distribution of Extreme Stock Market Returns", Journal of Business 69(3), 383-408.

[146] Longin F., (2005), "The Choice of the Distribution of Asset Returns: How Extreme Value Theory Can Help?", Journal of Banking E Finance 29(4), 1017-1035.

[147] Maillet B. and Th. Michel, (2003), "An Index of Market Shocks based on Multiscale Analysis", Quantitative Finance 3(2), 88-97.

[148] Maillet B. and Th. Michel, (2005), "The Impact of the 9/11 Events on the American and French Stock Markets", Review of International Economics 13(3), 597-611.

[149] Maillet B., J.-Ph. Médecin and Th. Michel, (2010), "High Watermarks of Market Risks", Journal of Mathematical Methods in Economics and Finance, forthcoming 2010, 21 pages.

[150] Malevergne Y. and D. Sornette, (2006), Extreme Financial Risks: From Dependence to Risk Management, Springer-Verlag, Birkhäuser, New York, 312 pages. 
[151] Malevergne Y., V. Pisarenko and D. Sornette, (2006), "On the Power of Generalized Extreme Value (GEV) and Generalized Pareto Distribution (GPD) Estimators for Empirical Distributions of Stock Returns", Applied Financial Economics 16(3), 271-289.

[152] Mancini C., (2009), "Non-parametric Threshold Estimation for Models with Stochastic Diffusion Coefficient and Jumps", Scandinavian Journal of Statistics 36(2), 270-296.

[153] Martens M. (2001), "Forecasting Daily Exchange Rate Volatility using intra-day Returns", Journal of International Money and Finance 20(1), $1-23$.

[154] Martens M., (2002), "Measuring and Forecasting S\&P500 Index-futures Volatility using High-frequency Data", Journal of Futures Markets 22(6), 497-518.

[155] Martens M. and D. van Dijk, (2007), "Measuring Volatility with the Realized Range", Journal of Econometrics 138(1), 181-207.

[156] Martens M., Y. Chang and S. Taylor, (2002), "A Comparison of Seasonal Adjustment Methods when Forecasting intra-day Volatility", Journal of Financial Research 25(2), 283-299.

[157] McDonald J., (1994), "Probability Distributions for Financial Models", in Handbook of Statistics, Vol. 14, Maddala-Rao Eds, Amsterdam Elsevier Science, 427-459.

[158] McLeod A. and W. Li, (1983), "Diagnostic Checking ARMA Time-series Models using Squared-residual Autocorrelations", Journal of Time Series Analysis 4(4), 269-273.

[159] McNeil A., (1998), "Calculating Quantile Risk Measures for Financial Return Series using Extreme Value Theory", Working Paper, ETH Zürich, 17 pages.

[160] McNeil A., R. Frey and P. Embrechts, (2005), Quantitative Risk Management: Concepts, Techniques, and Tools, Princeton Series in Finance, Princeton University Press, 538 pages.

[161] Martins-Filho C. and F. Yao, (2006), "Estimation of Value-at-Risk and Expected Shortfall based on Non-linear Models of Return Dynamics and Extreme Value Theory", Studies in Nonlinear Dynamics $\&$ Econometrics 10(2), 41 pages.

[162] Merton R., (1980), "On Estimating the Expected Return on the Market: An Exploratory Investigation", Journal of Financial Economics 8(4), 323361. 
[163] Miccichè S., G. Bonanno, F. Lillo and R. Mantegna, (2002), "Volatility in Financial Markets: Stochastic Models and Empirical Results", Physica A $314(1-4), 756-761$

[164] Modarres R., (2008), "Regional Maximum Wind Speed Frequency Analysis for the Arid and Semi-arid Regions of Iran", Journal of Arid Environments 72(7), 1329-1342.

[165] Moisello U., (2007), "On the Use of Partial Probability-weighted Moments in the Analysis of Hydrological Extremes", Hydrological Processes 21(10), 1265-1279.

[166] Moosa I. and B. Bollen, (2002), "A Benchmark for Measuring Bias in Estimated Daily Value-at-Risk", International Review of Financial Analysis 11(1), 85-100.

[167] Mudholkar G. and A. Hutson, (1998), "LQ-moments: Analogs of Lmoments", Journal of Statistical Planning and Inference 71(1-2), 191-208.

[168] Neftçi S., (2000), "Value-at-Risk Calculations, Extreme Events, and Tail Estimation", Journal of Derivatives 7(3), 23-37.

[169] Oomen R., (2004), "Modelling Realized Variance when Returns are Serially Correlated", Unpublished Manuscript, University of Warwick, May 2004, 35 pages.

[170] Oomen R., (2005), "Properties of Bias-corrected Realized Variance under Alternative Sampling Schemes", Journal of Financial Econometrics 3(4), $555-577$.

[171] Pandey M., P. van Gelder and J. Vrijling, (2001), "The Estimation of Extreme Quantiles of Wind Velocity using L-Moments in the Peaks-OverThreshold Approach", Structural Safety 23(2), 179-192.

[172] Parida B. and D. Moalafhi, (2008), "Regional Rainfall Frequency Analysis for Botswana using L-moments and Radial Basis Function Network", Physics and Chemistry of the Earth 33(8-13), 614-620.

[173] Parkinson M., (1980), "The Extreme Value Method for Estimating the Variance of the Rate of Return", Journal of Business 53(1), 61-65.

[174] Pickands J., (1975), "Statistical Inference using Extreme Order Statistics", Annals of Statistics 3(1), 119-131.

[175] Politis D. and J. Romano, (1994), "The Stationary Bootstrap", Journal of the American Statistical Association 89(428), 1303-1313.

[176] Politis D. and H. White, (2004), "Automatic Block-length Selection for the Dependent Bootstrap", Econometric Reviews 23(1), 53-70. 
[177] Poon S.-H. and C. Granger, (2003), "Forecasting Volatility in Financial Markets: A Review", Journal of Economic Literature 41(2), 478-539.

[178] Qi M. and Y. Wu, (2006), "Technical Trading-rule Profitability, Data Snooping, and Reality Check: Evidence from the Foreign Exchange Market", Journal of Money, Credit and Banking 38(8), 2135-2158.

[179] Reid N., (2000), "Likelihood", Journal of the American Statistical Association 95(452), 1335-1340.

[180] Resnick S., (1997), "Heavy-tail Modeling and Teletraffic Data", Annals of Statistics 25(5), 1805-1869.

[181] Robert C., J. Segers and C. Ferro, (2009), "A Sliding Blocks Estimator for the Extremal Index", Electronic Journal of Statistics 3, 993-1020.

[182] Robinson P., (1995), "Gaussian Semi-parametric Estimation of Long Range Dependence", Annals of Statistics 23(5), 1630-1661.

[183] Rogers L. and S. Satchell, (1991), "Estimating Variance from High, Low and Closing Prices", Annals of Applied Probability 1(4), 504-512.

[184] Rogers L., S. Satchell and Y. Yoon, (1994), "Estimating the Volatility of Stock Prices: A Comparison on Methods that use High and Low Prices", Applied Financial Economics 4(3), 241-247.

[185] Romano J. and M. Wolf, (2005), "Stepwise Multiple Testing as Formalized Data Snooping", Econometrica 73(4), 1237-1282.

[186] Saichev A., D. Sornette and V. Filimonov, (2009), "Most Efficient Homogeneous Volatility Estimators", Swiss Finance Institute Research Paper 09-35, 20 pages.

[187] Saichev A., D. Sornette, V. Filimonov and F. Corsi, (2010), "Homogeneous Volatility Bridge Estimators", Econometrics Journal 10, 1-25.

[188] Sankarasubramanian A. and K. Srinivasan, (1999), "Investigation and Comparison of Sampling Properties of L-moments and Conventional Moments", Journal of Hydrology 218(1-2), 13-34.

[189] Shabri A. and A. Jemain, (2007), "LQ-moments for Statistical Analysis of Extreme Events", Journal of Modern Applied Statistical Methods 6(1), 228-238.

[190] Shao Q., Y. Chen and L. Zhang, (2008), "An Extension of Threeparameter Burr III Distribution for Low-flow Frequency Analysis", Computational Statistics \& Data Analysis 52(3), 1304-1314.

[191] Schreiber T., (1998), "Constrained Randomization of Time-series Data", Physical Review Letters 80(10), 2105-2108. 
[192] Sillitto G., (1951), "Interrelations between Certain Linear Systematic Statistics of Samples from any Continuous Population", Biometrika 38(3-4), 377-382.

[193] Sillito G., (1969), "Derivation of Approximations to the Inverse Distribution Function of a Continuous Univariate Population from the Order Statistics of a Sample", Biometrika 56(3), 641-650.

[194] Silverman B., (1986), Density Estimation for Statistics and Data Analysis, Monographs on Statistics and Applied Probability 26, Chapman \& Hall/CRC, London, 176 pages.

[195] Thomakos D. and T. Wang, (2003), "Realized Volatility in the Futures Markets", Journal of Empirical Finance 10(3), 321-353.

[196] Thomas G., (2009), "Assessing Symmetry using Quantiles and Lmoments", Communications in Statistics: Simulation and Computation 38(1-2), 335-354.

[197] Thompson E., L. Baise and R. Vogel, (2007), "A Global Index Earthquake Approach to Probabilistic Assessment of Extremes", Journal of Geophysical Research 112(B06314), 12 pages.

[198] Tolikas K., (2008), "Value-at-Risk and Extreme Value Distributions for Financial Returns", Journal of Risk 10(3), 31-78.

[199] Tolikas K. and R. Brown, (2006), "The Distribution of the Extreme Daily Share Returns in the Athens Stock Exchange", European Journal of Finance 12(1), 1-22.

[200] Tolikas K., A. Koulakiotis and R. Brown, (2007), "Extreme Risk and Value-at-Risk in the German Stock Market", European Journal of Finance 13(4), 373-395.

[201] Tolikas K. and G. Gettinby, (2009), "Modelling the Distribution of the Extreme Share Returns in Singapore", Journal of Empirical Finance 16(2), 254-263.

[202] Vandewalle B., J. Beirlant, A. Christmann and M. Hubert, (2007), "A Robust Estimator for the Tail-index of Pareto-type Distributions", Computational Statistics 85 Data Analysis 51(12), 6252-6268.

[203] von Mises R., (1936), "La distribution de la plus grande de $n$ valeurs", Revue Mathematique de l'Union Interbalcanique 1 (1936), 141-160.

[204] Vuorenmaa T., (2008), "Decimalization, Realized Volatility, and Market Microstructure Noise", MPRA Working Paper \#8692, 58 pages.

[205] Wang Q., (1990), "Estimation of the GEV Distribution from Censored Samples by Method of Partial Probability-weighted Moments", Journal of Hydrology 120(1-4), 103-114. 
[206] Wang Q., (1997), "LH-moments for Statistical Analysis of Extreme Events", Water Resources Research 33(12), 2841-2848.

[207] Wells P., (2007), "The Rate of Profit as a Random Variable", Unpublished Ph.D. Thesis, University of London, 260 pages.

[208] Whalen T., G. Savage and G. Jeong, (2002), "The Method of Selfdetermined Probability-weighted Moments Revisited", Journal of Hydrology 268(1-4), 177-191.

[209] Whalen T., G. Savage and G. Jeong, (2004), "An Evaluation of the Self-determined Probability-weighted Moment Method for Estimating Extreme Wind Speeds", Journal of Wind Engineering and Industrial Aerodynamics 92(3-4), 219-239.

[210] White H., (2000), "A Reality Check for Data Snooping", Econometrica 68(5), 1097-1126.

[211] Whittle P., (1953), "Estimation and Information in Stationary Timeseries", Arkiv för Matematik 2(5), 423-434.

[212] Wiggins J., (1991), "Empirical Tests of the Bias and Efficiency of the Extreme-value Variance Estimator for Common Stocks", Journal of Business 64(3), 417-432.

[213] Yang D. and Q. Zhang, (2000), "Drift-independent Volatility Estimation based on High, Low, Open, and Close Prices", Journal of Business 73(3), 477-491.

[214] Zhang L., (2006), "Efficient Estimation of Stochastic Volatility using Noisy Observations: A Multi-scale Approach", Bernoulli 12(6), 1019-1043.

[215] Zhang L., P. Mykland and Y. Ait-Sahalia, (2005), "A Tale of Two Time Scales: Determining Integrated Volatility with Noisy High-frequency Data", Journal of the American Statistical Association 100(472), 13941411. 


\section{Appendices}

\section{Appendix A}

In the following, we give the proofs of the generalized formulae provided in Tables 1-a and 2-a that give the Tail-index expressions (for Shape-parameters strictly different from 0) for the GEV distribution and the GPD, as a function of the C-moments (Sankarasubramanian and Srinivasan, 1999 and Hosking and Wallis, 1987), the L-moments (Hosking, 1990; Abdul-Moniem and Selim, 2009), the TL-moments (Elamir and Seheult, 2003; Hosking, 2007; Abdul-Moniem and Selim, 2009), and the LQ-moments (Mudholkar and Hutson, 1998). We also present herein the (empirical) Self-determined Probability-weighted Moment (Sd-PwM) method (see Haktanir, 1997), corresponding to Table 1-b and 2-b. We will start by giving the proofs for the results summarized in Tables 1 that correspond to the GEV density, and we will adapt the proofs to the GPD for the results in Tables 2 just after. All the appendices are self-sufficient.

Let $\left\{X_{i}\right\}_{i \in[1, \ldots, n]}$ be a sequence of $n$ Independent and Identically Distributed non-degenerated random variables with a Quantile Function $Q_{X}($.$) defined by$ a continuous Cumulative Distribution Function $F($.$) on u \in] 0 ; 1[$, such as:

$$
Q_{X}(u)=F^{-1}(u) .
$$

We also note $X_{[i: n]}$ the $i$-th order statistic of $n$ (descending ordered) random variables. We here underline that the generic variable $X$ in the following corresponds to the highest Log-volatilities (or Log-volatility exceedances), denoted $\sigma$ in the corpus of the article.

We shall first recall the definitions of the various $r$-th moments, with $r \in$ $I N^{*}$, namely the L-moments, LH-moments, TL-moments and LQ-moments, that can be defined as follows.

Definitions 1 (about various L-moments definitions).

- The $r$-th L-moment is:

$$
\lambda_{r}=r^{-1} \sum_{j=0}^{r-1}(-1)^{j}\left(\begin{array}{r}
r-1 \\
j
\end{array}\right) E\left(X_{[r-j: r]}\right) .
$$

- The $r$-th TL-moment writes:

$$
\lambda_{r}^{(s, t)}=r^{-1} \sum_{j=0}^{r-1}(-1)^{j}\left(\begin{array}{l}
r-1 \\
j
\end{array}\right) E\left(X_{[r+s-j: r+s+t]}\right) .
$$

- The $r$-th LQ-moment reads:

$$
\lambda_{r}^{Q}=r^{-1} \sum_{j=0}^{r-1}(-1)^{j}\left(\begin{array}{l}
r-1 \\
j
\end{array}\right) \tau_{p, \alpha}\left(\hat{X}_{[r-j: r]}\right),
$$


where:

$$
\begin{gathered}
\tau_{p, \alpha}\left(\hat{X}_{[r-j: r]}\right)=p Q_{X_{[r-j: r]}(\alpha)+p Q_{X_{[r-j: r]}}(1-\alpha)}+(1-2 p) Q_{X_{[r-j: r]}}(.5)
\end{gathered}
$$

with $\hat{X}_{[r-j: r]}$ is the observed order statistic of order $(r-j, r) \in I N^{* 2}, Q_{X_{[r-j: r]}}($. the Quantile Function related to the order statistic $X_{[r-j: r]}$, and some parameters $(p, \alpha) \in[0,1]^{2}$.

As explained in Mudholkar and Hutson (1998), the linear function $\tau_{p, \alpha}()$ is a quick measure of the location of the sampling distribution of the order statistic $X_{[r-j: r]}$. We will only consider here that $\tau_{p, \alpha}($.$) is defined with p=\alpha=.25$, which corresponds to the common estimator called Trimean.

Definitions 2 (about L-moments, LH-moments, LL-moments and TL-moments or order $\left.r \in I N^{*}\right)$.

We note here from the previous definitions that L-moments, LH-moments and LL-moments are just specific cases of TL-moments, with - respectively - no truncation, only left or alternatively only right deletion. In other words, the $r$-th L-moment $\lambda_{r}$, the $r$-th LH-moment $\lambda_{r}^{H}$ and the $r$-th LL-moment $\lambda_{r}^{L}$ are given by:

$$
\left\{\begin{array}{c}
\lambda_{r}=\lambda_{r}^{(0,0)} \\
\lambda_{r}^{H}=\lambda_{r}^{(0, t)} \\
\lambda_{r}^{L}=\lambda_{r}^{(s, 0)} .
\end{array}\right.
$$

Accordingly we will then only present proofs for the results related to the TL-moments in the following (except for the L-moment expression of the GPD we need in the Sd-PwM method in Proposition 9 below) - see Table 1-a and 2-a for specific results for the L-moments, the LH-moments and the LL-moments).

Definitions 3 (about Extreme Value Densities and Quantile Functions).

Let us now recall the expressions of Cumulative Distribution and Quantile Functions related to both GEV density and GPD.

A GEV CDF of highest Log-volatilities $X$ is defined by three parameters: $v \in \mathbb{R}$, the location parameter, $\alpha \in \mathbb{R}_{+}$, the scale parameter, and $\xi \in \mathbb{R}^{*}$ the Shape-parameter, and writes:

$$
H(X)=\exp \left\{-\left[1+\xi_{\alpha}^{-1}(X-v)\right]^{-1 / \xi}\right\},
$$

for every $X \in \mathcal{D}_{1}$, defined by:

$$
\mathcal{D}_{1}= \begin{cases}]-\infty ; v-\xi_{\alpha}[ & \text { if } \xi<0 \\ \mathbb{R} & \text { if } \xi=0 \\ ] v-\xi_{\alpha} ;+\infty[ & \text { if } \xi>0,\end{cases}
$$

with, perconvention, $\xi_{\alpha}=\alpha / \xi$. 
The Quantile Function corresponding to a GEV density is then, for every $u \in] 0 ; 1[$ related to the variable $X$ (with the previous notations):

$$
Q_{X}(u)=v+\xi_{\alpha}\left\{[-\ln (u)]^{-\xi}-1\right\} .
$$

A GPD CDF of Log-volatility exceedances $X$ is defined by three parameters: $v \in \mathbb{R}$, the location parameter, $\alpha \in \mathbb{R}_{+}$, the scale parameter and $\xi \in \mathbb{R}^{*}$ the Shape-parameter and reads:

$$
H(X)=1-\left[1+\xi_{\alpha}^{-1}(X-v)\right]^{-1 / \xi},
$$

for every $X \in \mathcal{D}_{2}$, defined by:

$$
\mathcal{D}_{2}= \begin{cases}] v ; v-\xi_{\alpha}[ & \text { if } \xi<0 \\ ] v ;+\infty[ & \text { if } \xi \geq 0\end{cases}
$$

with $\xi_{\alpha}=\alpha / \xi$.

Then, the Quantile Function of a GPD is defined such as, for every $u \in] 0 ; 1[$ related to the variable $X$ (with the previous notations):

$$
Q_{X}(u)=v+\xi_{\alpha}\left[(1-u)^{-\xi}-1\right] .
$$

In the following, we will give results for GEV CDF and GPD CDF only in the case $\xi \neq 0$, since we are interested in the estimation of this last parameter.

Let us start now by recalling two properties related to L-moments and TLmoments that we will use later on.

Proposition 1 (see Hosking, 1990). The first three L-moments and Probabilityweighted Moments are linked by the following relations:

$$
\left\{\begin{array}{l}
\lambda_{1}=\beta_{0} \\
\lambda_{2}=2 \beta_{1}-\beta_{0} \\
\lambda_{3}=6 \beta_{2}-6 \beta_{1}+\beta_{0},
\end{array}\right.
$$

where $\lambda_{r}$ is the $r$-th L-moment and $\beta_{j}$ the $j$-th Probability-weighted Moment, with $(r, j) \in\{1,2,3\} \times\{0,1,2\}$.

Proof.

The first three Probability-weighted Moments are defined such as $\beta_{j}=$ $E\left\{X[F(X)]^{j}\right\}$ with $j \in\{0,1,2\}$.

Since the expectation of the order statistics can be written such as:

$$
\begin{array}{rl}
E\left(X_{[i: n]}\right)=\frac{r !}{(i-1) !(n-i) !} \int_{\mathfrak{D}} & x F(x)^{i-1}[1 \\
& -F(x)]^{n-i} d F(x),
\end{array}
$$

we immediately obtain the desired relations for first L-moment $\lambda_{1}$ : 


$$
\begin{aligned}
\lambda_{1} & =E\left(X_{[1: 1]}\right) \\
& =E(X)=\beta_{0},
\end{aligned}
$$

for the second L-moment $\lambda_{2}$ :

$$
\begin{aligned}
\lambda_{2} & =2^{-1}\left[E\left(X_{[2: 2]}\right)-E\left(X_{[1: 2]}\right)\right] \\
& =\int_{\mathfrak{D}} x[2 F(x)-1] d F(x)=2 \beta_{1}-\beta_{0},
\end{aligned}
$$

and for the third L-moment $\lambda_{3}$ :

$$
\begin{aligned}
\lambda_{3} & =3^{-1}\left[E\left(X_{[3: 3]}\right)-2 E\left(X_{[2: 3]}+E\left(X_{[1: 3]}\right)\right.\right. \\
& =\int_{\mathfrak{D}} x\left[6 F(x)^{2}-6 F(x)+1\right] d F(x) \\
& =6 \beta_{2}-6 \beta_{1}+\beta_{0} .
\end{aligned}
$$

The proposition below gives some useful relations between L-moments and TL-moments (that we will need herein).

Proposition 2 (see Hosking, 2007). L-moments and TL-moments are linked by the following relations:

$$
\left\{\begin{aligned}
\lambda_{r}^{(0, t)}= & \left(\frac{r+1}{2 r}\right)\left[\lambda_{r}^{(0,0)}-\lambda_{r+1}^{(0,0)}\right] \\
\lambda_{r}^{(0,2)}= & {\left[\frac{(r+1)(r+2)}{2 r(2 r+1)}\right] \lambda_{r}^{(0,0)}-\left(\frac{r+2}{2 r}\right) \lambda_{r+1}^{(0,0)} } \\
& +\left[\frac{r+2}{2(2 r+1)}\right] \lambda_{r+2}^{(0,0)} \\
\lambda_{r}^{(1,1)}= & {\left[\frac{(r+1)(r+2)}{2 r(2 r+1)}\right]\left[\lambda_{r}^{(0,0)}-\lambda_{r+2}^{(0,0)}\right], }
\end{aligned}\right.
$$

where $\lambda_{r}^{(s, t)}$ is the $r$-th TL-moment and $\lambda_{r}^{(0,0)}$ the corresponding L-moment, with $(r, s, t) \in\left(\mathbb{N}^{*} \times\{0,1\} \times \mathbb{N}\right)$.

Proof.

Indeed, recalling that (from the previous relations):

$$
\begin{aligned}
E\left(X_{[i: r]}\right)= & \frac{r !}{(i-1) !(r-i) !} \\
& \times \int_{0}^{1} Q_{X}(u) u^{i-1}(1-u)^{r-i} d u,
\end{aligned}
$$

the TL-moments of order $r$ can then be formally computed with the following expression:

$$
\begin{aligned}
\lambda_{r}^{(s, t)}= & r^{-1} \sum_{j=0}^{r-1}(-1)^{j}\left(\begin{array}{l}
r-1 \\
j
\end{array}\right) \\
& \times[(r+s-j-1) ! \\
& \times(t+j) !]^{-1}(r+s+t) ! \\
& \times \int_{0}^{1} Q_{X}(u) u^{r+s-j-1}(1-u)^{t+j} d u .
\end{aligned}
$$


We also note here that every $r$-th TL-moment can be written as a linear function of TL-moments of order 1 , such as:

$$
\lambda_{r}^{(s, t)}=r^{-1} \sum_{j=0}^{r-1}(-1)^{j}\left(\begin{array}{l}
r-1 \\
j
\end{array}\right) \lambda_{1}^{(r+s-j-1, t+j)}
$$

for any $(r, s, t) \in\left(\mathbb{N}^{*} \times \mathbb{N}^{2}\right)$.

This last relation will be of great help to prove some of following propositions, since it is sufficient to compute TL-moments of order 1 for obtaining all higher TL-moments.

In the following, we shall start with the proofs for the results in Tables 1 (GEV-related ones in the order of the table results), and then we will turn to those in Tables 2 (for the GDP, as well in the order of the table results).

Proposition 3 (see Sankarasubramanian and Srinivasan, 1999). The first three Conventional Moments, as a function of the three characteristic parameters of a GEV distribution, are given by:

$$
\left\{\begin{array}{r}
m_{1}=v-\xi_{\alpha}+\xi_{\alpha} g(\xi) \\
m_{2}=\left(\xi_{\alpha}\right)^{2}\left\{g(2 \xi)-[g(\xi)]^{2}\right\} \\
m_{3}=\left(\xi_{\alpha}\right)^{3}\{g(3 \xi)-3 g(2 \xi) g(\xi) \\
\left.+2[g(\xi)]^{3}\right\}
\end{array}\right.
$$

where $m_{r}$ is the $r$-th Conventional Moment with $r \in\{1,2,3\}, v \in \mathbb{R}$ is the location parameter, $\alpha \in \mathbb{R}_{+}$the scale parameter, $\xi \in \mathbb{R}^{*}$ the Shape-parameter, with $\xi_{\alpha}=\alpha / \xi, g()=.\Gamma[h()$.$] where h(k+j \xi)=(k+1-j \xi)$ for any $(j, k) \in$ $\{1,2,3\} \times I N$, and $\Gamma(\xi)=\int_{0}^{+\infty} t^{\xi-1} \exp (-t) d t$ the Gamma function.

Proof.

We first recall that the Beta function, denoted $B(.,$.$) , satisfies the following$ relation:

$$
B(s, t)=\int_{0}^{1} u^{s-1}(1-u)^{t-1} d u=\frac{\Gamma(s) \Gamma(t)}{\Gamma(s+t)},
$$

where $\Gamma(x)=\int_{0}^{+\infty} u^{x-1} \exp (-u) d u$ is the Gamma function.

Thanks to simple changes of variables, we also have the following intermediate result (with the previous notations):

$$
\begin{aligned}
\int_{0}^{1} u^{j}[-\ln (u)]^{-\xi} d u= & \int_{0}^{+\infty} x^{-\xi} \\
& \times \exp [-(j+1) x] d x \\
= & (1+j)^{-h(\xi)} \\
& \times \int_{0}^{+\infty} y^{-\xi} \exp (-y) d y \\
= & (1+j)^{-h(\xi)} g(\xi),
\end{aligned}
$$


with $g()=.\Gamma[h()$.$] where h(k+j \xi)=(k+1-j \xi)$ for any $(j, k) \in\{1,2,3\} \times$ $I N$.

For the first moment, we now have (with the previous notations):

$$
\begin{aligned}
m_{1} & =\int_{0}^{1} Q_{X}(u) d u \\
& =\int_{0}^{1} v-\xi_{\alpha}+\xi_{\alpha}[-\ln (u)]^{-\xi} d u .
\end{aligned}
$$

With the result (66), we obtain (with the previous notations):

$$
m_{1}=v-\xi_{\alpha}+\xi_{\alpha} g(\xi) .
$$

In the same way, the second moment writes (with the previous notations):

$$
\begin{aligned}
m_{2} & =\int_{0}^{1}\left[Q_{X}(u)-m_{1}\right]^{2} d u \\
& =\int_{0}^{1}\left(\xi_{\alpha}\right)^{2}\left\{[-\ln (u)]^{-\xi}-g(\xi)\right\}^{2} d u \\
& =\left(\xi_{\alpha}\right)^{2}\left\{g(2 \xi)-[g(\xi)]^{2}\right\} .
\end{aligned}
$$

Finally, the third moment is given by (with the previous notations):

$$
\begin{aligned}
m_{3} & =\int_{0}^{1}\left(\xi_{\alpha}\right)^{3}\left\{[-\ln (u)]^{-\xi}-g(\xi)\right\}^{3} d u \\
& =\left(\xi_{\alpha}\right)^{2}\left\{g(3 \xi)-3 g(2 \xi) g(\xi)+2[g(\xi)]^{3}\right\} .
\end{aligned}
$$

Proposition 4. The first three TL-moments, as a function of the three characteristic parameters of a GEV distribution, are given by:

$$
\left\{\begin{aligned}
\lambda_{1}^{(s, t)}= & \left(v-\xi_{\alpha}\right)+\frac{(1+s+t) !}{s ! t !} \xi_{\alpha} \\
& \times \sum_{k=0}^{t}(-1)^{k}(1+s+k)^{-h(\xi)} g(\xi) \\
\lambda_{2}^{(s, t)}= & 2^{-1} \xi_{\alpha} g(\xi)\left[-\frac{(2+s+t) !}{s !(t+1) !}(1+s)^{-h(\xi)}\right. \\
& +\frac{(2+s+t) !}{(s+1) !} \sum_{k=0}^{t}(-1)^{k} \frac{(2+k+s)}{(k+1) !(t-k) !} \\
& \left.\times(2+s+k)^{-h(\xi)}\right] \\
\lambda_{3}^{(s, t)}= & 3^{-1} \xi_{\alpha} g(\xi)\left[\frac{(3+s+t) !}{s !(2+t) !}(1+s)^{-h(\xi)}\right. \\
& -\frac{(3+s+t) !(3+s)}{(s+1) !(1+t) !}(2+s)^{-h(\xi)} \\
& +\frac{(3+s+t) !}{(2+s) !} \sum_{k=0}^{t}(-1)^{k} \frac{(3+k+s)(4+k+s)}{(2+k) !(t-k) !} \\
& \left.\times(3+s+k)^{-h(\xi)}\right]
\end{aligned}\right.
$$

where $\lambda_{r}^{(s, t)}$ is the $r$-th TL-moment of truncation order $(s, t)$ for any $(r, s, t)$ $\in\{1,2,3\} \times I N^{2}, v \in \mathbb{R}$ the location parameter, $\alpha \in \mathbb{R}_{+}$the scale parameter, 
$\xi \in \mathbb{R}^{*}$ the Shape-parameter, with $\xi_{\alpha}=\alpha / \xi, g()=.\Gamma[h()$.$] where h(k+j \xi)=$ $(k+1-j \xi)$ for any $(j, k) \in\{1,2,3\} \times I N$, and $\Gamma(\xi)=\int_{0}^{+\infty} u^{\xi-1} \exp (-u) d u$ the Gamma function.

Proof.

From the Quantile Function corresponding to a GEV density (equation (52)), we can then compute the first TL-moment such as (with the previous notations):

$$
\begin{aligned}
\lambda_{1}^{(s, t)}= & \frac{(1+s+t) !}{s ! t !} \int_{0}^{1} Q_{X}(u) u^{s}(1-u)^{t} d u \\
= & \frac{(1+s+t) !}{s ! t !}\left[\int_{0}^{1}\left(v-\xi_{\alpha}\right) u^{s}(1-u)^{t} d u\right. \\
& \left.+\xi_{\alpha} \int_{0}^{1}[-\ln (u)]^{-\xi} u^{s}(1-u)^{t} d u\right] .
\end{aligned}
$$

Using results (65) and (66), we obtain the final expression for the first TLmoment as such (with the previous notations):

$$
\begin{aligned}
\lambda_{1}^{(s, t)}= & \frac{(1+s+t) !}{s ! t !}\left[\frac{\Gamma(s+1) \Gamma(t+1)}{\Gamma(2+s+t)}\right. \\
& \left.+\xi_{\alpha} \sum_{k=0}^{t}(-1)^{k}\left(\begin{array}{l}
0 \\
k
\end{array}\right) \int_{0}^{1}[-\ln (u)]^{-\xi} u^{k+s} d u\right] \\
= & v-\xi_{\alpha}+\frac{(1+s+t) !}{s ! t !} \\
& \times \xi_{\alpha} \sum_{k=0}^{t}(-1)^{k}(1+k+s)^{-h(\xi)} g(\xi) .
\end{aligned}
$$

Thanks to relation (63), we similarly get the second TL-moment as such (with the previous notations):

$$
\begin{aligned}
\lambda_{2}^{(s, t)}= & 2^{-1}\left[\lambda_{1}^{(1+s, t)}-\lambda_{1}^{(s, t+1)}\right] \\
= & 2^{-1}\left\{\left[\left(v-\xi_{\alpha}\right)+\frac{(2+s+t) !}{(s+1) ! t !} \xi_{\alpha}\right.\right. \\
& \left.\times \sum_{k=0}^{t}(-1)^{k}\left(\begin{array}{c}
t \\
k
\end{array}\right)(2+k+s)^{-h(\xi)} g(\xi)\right] \\
& -\left[\left(v-\xi_{\alpha}\right)+\frac{(2+s+t) !}{s !(t+1) !} \xi_{\alpha}\right. \\
& \times \sum_{k=0}^{t+1}(-1)^{k}\left(\begin{array}{c}
t+1 \\
k
\end{array}\right) \\
& \left.\left.\times(1+k+s)^{-h(\xi)} g(\xi)\right]\right\} \\
= & 2^{-1} \xi_{\alpha} g(\xi)\left[-\frac{(2+s+t) !}{s !(t+1) !}(1+s)^{-h(\xi)}\right. \\
& +\frac{(2+s+t) !}{(s+1) !} \times \sum_{k=0}^{t}(-1)^{k} \\
& \left.\times \frac{(2+k+s)}{(k+1) !(t-k) !}(2+k+s)^{-h(\xi)}\right] .
\end{aligned}
$$

The third TL-moment is obtained in the same manner with the relation (63) as 
such (with the previous notations):

$$
\begin{aligned}
\lambda_{3}^{(s, t)}= & 3^{-1}\left[\lambda_{1}^{(2+s, t)}-2 \lambda_{1}^{(1+s, t+1)}+\lambda_{1}^{(s, t+2)}\right] \\
= & 3^{-1}\left\{\left(v-\xi_{\alpha}\right)+\frac{(3+s+t) !}{(2+s) ! t !} \xi_{\alpha}\right. \\
& \times \sum_{k=0}^{t}(-1)^{k}\left(\begin{array}{c}
t \\
k
\end{array}\right)(3+k+s)^{-h(\xi)} g(\xi) \\
& -2\left[\left(v-\xi_{\alpha}\right)+\frac{(3+s+t) !}{(1+s) !(1+t) !} \xi_{\alpha}\right. \\
& \left.\times \sum_{k=0}^{t+1}(-1)^{k}\left(\begin{array}{c}
t+1 \\
k
\end{array}\right)(2+k+s)^{-h(\xi)} g(\xi)\right] \\
& +\left(v-\xi_{\alpha}\right)+\frac{(3+s+t) !}{s !(2+t) !} \xi_{\alpha} \\
& \left.\times \sum_{k=0}^{t+2}(-1)^{k}\left(\begin{array}{c}
t+2 \\
k
\end{array}\right)(1+k+s)^{-h(\xi)} g(\xi)\right\} .
\end{aligned}
$$

Hence, we can write the following equality as such (with the previous notations):

$$
\begin{aligned}
\lambda_{3}^{(s, t)}=3^{-1} \xi_{\alpha} & g(\xi)\left\{\frac{(3+s+t) !}{(2+s) ! t !} \sum_{k=0}^{t}(-1)^{k}\left(\begin{array}{l}
t \\
k
\end{array}\right)\right. \\
\times & (3+k+s)^{-h(\xi)} \\
- & 2\left[\frac{(3+s+t) !}{(1+s) !(1+t) !}(2+s)^{-h(\xi)}\right. \\
& +\frac{(3+s+t) !}{(1+s) !(1+t) !} \sum_{k=0}^{t}(-1)^{k+1}\left(\begin{array}{c}
t+1 \\
k+1
\end{array}\right) \\
& \left.\times(3+k+s)^{-h(\xi)}\right] \\
& +\frac{(3+s+t) !}{s !(2+t) !}(1+s)^{-h(\xi)} \\
& -\frac{(3+s+t) !(t+2)}{s !(2+t) !}(2+s)^{-h(\xi)} \\
& +\frac{(3+s+t) !}{s !(2+t) !} \sum_{k=0}^{t}(-1)^{k}\left(\begin{array}{c}
t+2 \\
k+2
\end{array}\right) \\
& \left.\times(3+k+s)^{-h(\xi)}\right\} .
\end{aligned}
$$

Proposition 5 (see Hosking et al., 1985). The first three L-moments, as a function of the three characteristic parameters of a GEV distribution, are given by:

$$
\left\{\begin{array}{l}
\lambda_{1}=v-\xi_{\alpha}+\xi_{\alpha} g(\xi) \\
\lambda_{2}=\left(\xi_{\alpha}\right)^{2}\left\{g(2 \xi)-[g(\xi)]^{2}\right\} \\
\lambda_{3}=\left(\xi_{\alpha}\right)^{3}\left\{g(3 \xi)-3 g(2 \xi) g(\xi)+2[g(\xi)]^{3}\right\},
\end{array}\right.
$$

where $\lambda_{r}$ is the $r$-th L-moment with $r \in\{1,2,3\}, v \in \mathbb{R}$ the location parameter, $\alpha \in \mathbb{R}_{+}$the scale parameter, $\xi \in \mathbb{R}^{*}$ the Shape-parameter, and with $\xi_{\alpha}=\alpha / \xi, g()=.\Gamma[h()$.$] where h(k+j \xi)=(k+1-j \xi)$ for any $(j, k) \in$ $\{1,2,3\} \times I N$, and $\Gamma(\xi)=\int_{0}^{+\infty} u^{\xi-1} \exp (-u) d u$ the Gamma function.

Proof.

The proof is a direct consequence of Proposition 4 by taking $s=t=0$.

Proposition 6 (see Hosking et al., 1985). The first three Probability-weighted Moments are related to the parameters of a GEV distribution by the following relations: 


$$
\left\{\begin{array}{l}
\frac{\left[3 \beta_{2}-\beta_{0}\right]}{\left[2 \beta_{1}-\beta_{0}\right]}-\frac{\left[1-3^{\xi}\right]}{\left[1-2^{\xi}\right]}=0 \\
\alpha=\left(\beta_{0}-2 \beta_{1}\right)\left[g(\xi)\left(1-2^{\xi}\right)\right] \xi \\
v=\beta_{0}+\xi_{\alpha}[1-g(\xi)],
\end{array}\right.
$$

where the $\beta_{j}$ are the Probability-weighted Moments with $j \in\{0,1,2\}, v \in \mathbb{R}$ the location parameter, $\alpha \in \mathbb{R}_{+}$the scale parameter, $\xi \in \mathbb{R}^{*}$ the Shape-parameter, and with $\xi_{\alpha}=\alpha / \xi, g()=.\Gamma[h()$.$] where h(k+j \xi)=(k+1-j \xi)$ for any $(j, k) \in\{1,2,3\} \times I N$, and $\Gamma(\xi)=\int_{0}^{+\infty} u^{\xi-1} \exp (-u) d u$ the Gamma function.

Proof.

The proof is a direct consequence of Propositions 1 and 5 .

Proposition 7 (see Mudholkar and Hutson, 1998). For any distribution $F($. of the variable $X$, defined by its related Quantile Function $Q_{X}($.$) , the first three$ $L Q$-moments are given by:

$$
\left\{\begin{aligned}
\lambda_{1}^{Q}= & 4^{-1}\left[Q_{X}(.25)+Q_{X}(.75)+2 Q_{X}(.5)\right] \\
\lambda_{2}^{Q}= & 8^{-1}\left[2 Q_{X}(.707)-2 Q_{X}(.293)\right. \\
& \left.Q_{X}(.866)-Q_{X}(.134)\right] \\
\lambda_{3}^{Q}= & 12^{-1}\left[Q_{X}(.909)+2 Q_{X}(.794)\right. \\
& -2 Q_{X}(.674)+Q_{X}(.630) \\
& -4 Q_{X}(.5)+Q_{X}(.370) \\
& -2 Q_{X}(.326)+2 Q_{X}(.206) \\
& \left.+Q_{X}(.091)\right] .
\end{aligned}\right.
$$

Proof.

Using the usual formula for the CDF of order statistics, denoted $F_{X_{[i: n]}}($.$) ,$ for every $n \in \mathbb{N}^{*}$ and $i \in\{1, \ldots, n\}$,

$$
F_{X_{[i: n]}}(x)=\sum_{k=i}^{n}\left(\begin{array}{l}
n \\
k
\end{array}\right) F_{X}^{k}(x)\left[1-F_{X}(x)\right]^{n-k},
$$

we obtain the following Quantile Function - denoted $Q_{X_{[i: n]}}($.$) - relations for$ some specific order statistics, written $X_{[i: n]}$ for some $(i, n) \in\{1,2,3\}^{2}$, as such:

$$
\left\{\begin{array}{l}
Q_{X_{[1: 2]}}(u)=Q_{X}(1-\sqrt{1-u}) \\
Q_{X_{[2: 2]}}(u)=Q_{X}(\sqrt{u}) \\
Q_{X_{[1: 3]}}(u)=Q_{X}(1-\sqrt[3]{1-u}) \\
Q_{X_{[3: 3]}}(u)=Q_{X}(\sqrt[3]{u}) \\
Q_{X_{[2: 3]}}(u)=Q_{X}[\varphi(u)]
\end{array}\right.
$$

where $\varphi($.$) is the unique root in [0 ; 1]$ of the equation $\left(2 x^{3}-3 x^{2}+u\right)=0$. 
In the case where the LQ-moments are defined with the Trimean, we have:

$$
\begin{aligned}
\tau\left(X_{[r-j: r]}\right)= & 4^{-1} Q_{X_{[r-j: r]}(.25)} \\
& +2^{-1} Q_{X_{[r-j: r]}}(.5) \\
& +4^{-1} Q_{X_{[r-j: r]}}(.75),
\end{aligned}
$$

and the first three LQ-moments are given by:

$$
\left\{\begin{aligned}
\lambda_{1}^{Q}= & 4^{-1}\left[Q_{X}(.25)+Q_{X}(.75)+2 Q_{X}(.5)\right] \\
\lambda_{2}^{Q}= & 8^{-1}\left[2 Q_{X}(.707)-2 Q_{X}(.293)\right. \\
& \left.+Q_{X}(.866)-Q_{X}(.134)\right] \\
\lambda_{3}^{Q}= & 12^{-1}\left[Q_{X}(.909)+2 Q_{X}(.794)\right. \\
& -2 Q_{X}(.674)+Q_{X}(.630) \\
& -4 Q_{X}(.5)+Q_{X}(.370) \\
& -2 Q_{X}(.326)+2 Q_{X}(.206) \\
& \left.+Q_{X}(.091)\right] .
\end{aligned}\right.
$$

From the above definitions of the GEV-related Quantile Function as well as the GPD-related one, it is then straightforward to obtain, by substitution, the first three LQ-moments for the GEV distribution (see Table 1-a) and for the GPD (see Table 2-a) as presented in the following Proposition 8.

Proposition 8. The first three LQ-moments, as a function of the three characteristic parameters of - respectively - a GEV distribution and a GPD, are:

$$
\left\{\begin{aligned}
\lambda_{1}^{Q}= & v-\xi_{\alpha}+4^{-1} \xi_{\alpha}[\check{Q}(.25) \\
& +\check{Q}(.75)+2 \check{Q}(.5)] \\
\lambda_{2}^{Q}= & 8^{-1} \xi_{\alpha}[2 \check{Q}(.707)-2 \check{Q}(.293) \check{Q}(.866) \\
\lambda_{3}^{Q}= & 12^{-1} \xi_{\alpha}[\check{Q}(.909)+2 \check{Q}(.794)-\check{Q}(.134)] \\
& -2 \check{Q}(.674)+\check{Q}(.630) \\
& -4 \check{Q}(.5)+\check{Q}(.370) \\
& -2 \check{Q}(.326)+2 \check{Q}(.206)+\check{Q}(.091)],
\end{aligned}\right.
$$

where $v \in \mathbb{R}_{+}$is the location parameter, $\alpha \in \mathbb{R}_{+}$the scale parameter, $\xi \in \mathbb{R}^{*}$ the Shape-parameter, with $\xi_{\alpha}=\alpha / \xi$ and $\check{Q}(u)=[-\ln (u)]^{-\xi}$ for a GEV density and $\check{Q}(u)=(1-u)^{-\xi}$ for a $G P D$.

Proof.

The proof is a direct consequence of the previous Proposition 7 by replacing $Q_{X}(u)$ by an adequate truncated expression for a GEV distribution such as $\check{Q}(u)=[-\ln (u)]^{-\xi}$, or by an appropriate truncated expression for a GPD distribution such as $\check{Q}(u)=(1-u)^{-\xi}$. Evaluating the various quantile-related quantities finally leads to results in Table 1-a and Table 2-a for the LQ-moments. 
Proposition 9 (see Haktanir, 1997). The estimates $\hat{v}, \hat{\alpha}$ and $\hat{\xi}$, of the three characteristic parameters of a $G E V$ distribution, i.e. $v \in \mathbb{R}$ the location parameter, $\alpha \in \mathbb{R}_{+}$the scale parameter and $\xi \in \mathbb{R}^{*}$ the Shape-parameter, are obtained with the Self-determined Probability-weighted Moment method as the solution of the following system:

$$
\left\{\begin{array}{c}
\frac{\bar{X}-3 n^{-1} \sum_{i=1}^{n}\left\{\hat{X}_{[i: n]}^{2}\left[\hat{H}\left(\hat{X}_{[i: n]}\right)\right]^{2}\right\}}{\bar{X}-2 n^{-1} \sum_{i=1}^{n}\left[\hat{X}_{[i: n]} \hat{H}\left(\hat{X}_{[i: n]}\right)\right]} \\
-\frac{1-3}{1-2 \hat{\xi}} \\
\hat{\alpha}=\frac{\left\{\bar{X}-2 n^{-1} \sum_{i=1}^{n}\left[\hat{X}_{[i: n]} \hat{H}\left(\hat{X}_{[i: n]}\right)\right]\right\}}{\left[g(\hat{\xi})\left(1-2^{\hat{\xi}}\right)\right]} \hat{\xi} \\
\hat{v}=\bar{X}+\hat{\alpha}[1-g(\hat{\xi})] \hat{\xi}^{-1},
\end{array}\right.
$$

where $\left\{\hat{X}_{[i: n]}\right\}_{i \in[1, \ldots, n]}$ are the empirical order statistics of the highest Logvolatilities, $\hat{H}\left(\hat{X}_{[i: n]}\right)=\exp \left\{-\left\{-\hat{\xi}_{\hat{\alpha}}^{-1}\left[\bar{X}-\hat{X}_{[i: n]}+g(\hat{\xi})\right]\right\}^{-1 / \hat{\xi}}\right\}$ is the empirical estimate of a GEV CDF of estimated order statistics, and with $\bar{X}=$ $n^{-1} \sum_{i=1}^{n} \hat{X}_{[i: n]}, \hat{\xi}_{\hat{\alpha}}=\hat{\alpha} / \hat{\xi}, g()=.\Gamma[h()$.$] where h(k+j \xi)=(k+1-j \xi)$ for any $(j, k) \in\{1,2,3\} \times I N$, and $\Gamma(\xi)=\int_{0}^{+\infty} u^{\xi-1} \exp (-u) d u$ the Gamma function.

Proof.

Considering the relations between the first three Probability-weighted Moments and the parameters of a GEV distribution given in Proposition 6, it is now simply necessary to recover some estimates of the $\beta_{j}$ from the sample. The usual empirical estimate of any $\hat{\beta}_{j}$ is given by (with the previous notations):

$$
\hat{\beta}_{j}=n^{-1} \sum_{i=1}^{n}\left(\hat{X}_{[i: n]} \operatorname{Pnex}_{i, j}\right)
$$

where $\operatorname{Pnex}_{i, j}=\left[\hat{F}\left(\hat{X}_{[i: n]}\right)\right]^{j}$ is an empirical distribution-free estimate of the CDF of order statistics to the power $j$ such as:

$$
\text { Pnex }_{i, j}= \begin{cases}1 & \text { if } j=0 \\ (i-1)(n-1)^{-1} & \text { if } j=1 \\ (i-1)(i-2)[(n-1)(n-2)]^{-1} & \text { if } j=2 .\end{cases}
$$

However, in the special case of the Sd-PwM method, estimates of order statistic probabilities correspond to the supposed Extreme Value CDF, which 
is, in our case here, the GEV CDF whose estimate writes (with the previous notations):

$$
\hat{H}\left(\hat{X}_{[i: n]}\right)=\exp \left\{-\left[1+\hat{\xi}_{\hat{\alpha}}^{-1}\left(\hat{X}_{[i: n]}-\hat{v}\right)\right]^{-1 / \hat{\xi}}\right\} .
$$

Replacing in this equation the location parameter $\hat{v}$ by its estimated value from equation (78) leads to (with the previous notations):

$$
\hat{H}\left(\hat{X}_{[i: n]}\right)=\exp \left\{-\left[-\hat{\xi}_{\hat{\alpha}}^{-1}\left(\bar{X}-\hat{X}_{[i: n]}\right)+g(\hat{\xi})\right]^{-1 / \hat{\xi}}\right\} .
$$

Combined with the equations (78), this last equation leads to the desired system.

Solving it finally allows us to retrieve from the data the value of the parameter $\hat{\xi}$, altogether with $\hat{\alpha}$ and $\hat{v}$.

After having given the main results for the GEV in the above part of the appendix (for the results in Tables 1), let us now turn below to the GDP case (see Tables 2).

Proposition 10 (see Hosking and Wallis, 1987). The first three Conventional Moments, as a function of the three characteristic parameters of a GPD, are given by:

$$
\left\{\begin{array}{l}
m_{1}=v+\alpha[h(\xi)]^{-1} \\
m_{2}=\alpha^{2}\left\{[h(\xi)]^{2} h(2 \xi)\right\}^{-1} \\
m_{3}=2 \alpha^{3}(1+\xi)\left\{[h(\xi)]^{3} h(2 \xi) h(3 \xi)\right\}^{-1},
\end{array}\right.
$$

where $m_{r}$ is the $r$-th Conventional Moment with $r=\{1,2,3\}, v \in \mathbb{R}$ is the location parameter, $\alpha \in \mathbb{R}_{+}$the scale parameter, $\xi \in \mathbb{R}^{*}$ the Shape-parameter, and $h(k+j \xi)=(k+1-j \xi)$ for any $(j, k) \in\{1,2,3\} \times I N$.

Proof.

The first moment is such as (with the previous notations):

$$
\begin{aligned}
m_{1} & =\int_{0}^{1} Q_{X}(u) d u \\
& =\int_{0}^{1}\left[v-\xi_{\alpha}+\xi_{\alpha}(1-u)^{-\xi}\right] d u \\
& =v+\alpha[h(\xi)]^{-1} .
\end{aligned}
$$


The second moment writes (with the previous notations):

$$
\begin{aligned}
m_{2}= & \int_{0}^{1}\left[Q_{X}(u)-m_{1}\right]^{2} d u \\
= & \int_{0}^{1}\left(\xi_{\alpha}\right)^{2}\left\{(1-u)^{-\xi}-[h(\xi)]^{-1}\right\}^{2} d u \\
= & \int_{0}^{1}\left(\xi_{\alpha}\right)^{2}\left\{-\frac{(1-u)^{h(2 \xi)}}{h(2 \xi)}+\frac{u}{[h(\xi)]^{2}}\right. \\
& \left.+\frac{2(1-u)^{h(\xi)}}{[h(\xi)]^{2}}\right\} d u \\
= & \alpha^{2}\left\{[h(\xi)]^{2} h(2 \xi)\right\}^{-1} .
\end{aligned}
$$

Finally, the third moment is given by (with the previous notations):

$$
\begin{aligned}
m_{3}= & \int_{0}^{1}\left[Q_{X}(u)-m_{1}\right]^{3} d u \\
= & \int_{0}^{1}\left(\xi_{\alpha}\right)^{3}\left\{(1-u)^{-\xi}-[h(\xi)]^{-1}\right\}^{3} d u \\
= & \int_{0}^{1}\left(\xi_{\alpha}\right)^{3}\left\{-\frac{(1-u)^{h(3 \xi)}}{h(3 \xi)}-\frac{u}{[h(\xi)]^{3}}\right. \\
& \left.-\frac{3(1-u)^{h(\xi)}}{[h(\xi)]^{3}}+\frac{3(1-u)^{h(2 \xi)}}{[h(\xi) h(2 \xi)]}\right\} d u \\
= & 2 \alpha^{3}(1+\xi)\left\{[h(\xi)]^{3} h(2 \xi) h(3 \xi)\right\}^{-1} .
\end{aligned}
$$

Proposition 11. The first three TL-moments, as a function of the three characteristic parameters of a GPD, are given by:

$$
\begin{cases}\lambda_{1}^{(s, t)}= & v-\xi_{\alpha}+\frac{(1+s+t) !}{t !} \frac{g(t+\xi)}{g(1+s+t+\xi)} \xi_{\alpha} \\ \lambda_{2}^{(s, t)}= & \frac{(2+s+t) !}{2(t+1) !} \frac{g(t+\xi)}{g(2+s+t+\xi)} \alpha \\ \lambda_{3}^{(s, t)}= & \frac{(3+s+t) !}{3(t+2) !} \frac{g(t+\xi)}{g(3+s+t+\xi)} \alpha(1+\xi),\end{cases}
$$

where $\lambda_{r}^{(s, t)}$ is the $r$-th TL-moment of truncation order $(s, t)$ for any $(r, s, t) \in$ $\{1,2,3\} \times I N^{2}, v \in \mathbb{R}$ the location parameter, $\alpha \in \mathbb{R}_{+}$the scale parameter, $\xi \in$ $\mathbb{R}^{*}$ the Shape-parameter, and with $\xi_{\alpha}=\alpha / \xi, g()=.\Gamma[h()$.$] where h(k+j \xi)=$ $(k+1-j \xi)$ for any $(j, k) \in\{1,2,3\} \times I N$, and $\Gamma(\xi)=\int_{0}^{+\infty} u^{\xi-1} e^{-u} d u$ the Gamma function.

Proof.

The first TL-moment reads (with the previous notations):

$$
\begin{aligned}
\lambda_{1}^{(s, t)}= & \frac{(1+s+t) !}{s ! t !} \int_{0}^{1} Q_{X}(u) u^{s}(1-u)^{t} d u \\
= & \frac{(1+s+t) !}{s ! t !}\left[\int_{0}^{1}\left(v-\xi_{\alpha}\right) u^{s}(1-u)^{t} d u\right. \\
& \left.+\xi_{\alpha} \int_{0}^{1} u^{s}(1-u)^{t-\xi} d u\right] .
\end{aligned}
$$


With equation (65), we have the following result for the first TL-moment (with the previous notations):

$$
\begin{aligned}
\lambda_{1}^{(s, t)}= & \frac{(1+s+t) !}{s ! t !}\left[\left(v-\xi_{\alpha}\right) \frac{\Gamma(s+1) \Gamma(t+1)}{\Gamma(2+s+t)}\right. \\
& \left.+\xi_{\alpha} \frac{\Gamma(s+1) g(t+\xi)}{g(1+s+t+\xi)}\right] \\
= & \left(v-\xi_{\alpha}\right)+\frac{(1+s+t) !}{t !} \\
& \times \frac{g(t+\xi)}{g(1+s+t+\xi)} \xi_{\alpha} .
\end{aligned}
$$

Thanks to relation (63), we get the second TL-moment expression as such (with the previous notations):

$$
\begin{aligned}
\lambda_{2}^{(s, t)}= & \frac{1}{2}\left[\lambda_{1}^{(1+s, t)}-\lambda_{1}^{(s, t+1)}\right] \\
= & 2^{-1} \xi_{\alpha}\left[\frac{(2+s+t) !}{t !} \frac{g(t+\xi)}{g(2+s+t+\xi)}\right. \\
& \left.-\frac{(2+s+t) !}{(t+1) !} \frac{g(t+1+\xi)}{g(2+s+t+\xi)}\right] \\
= & \frac{(2+s+t) !}{2(t+1) !} \frac{g(t+\xi)}{g(2+s+t+\xi)} \alpha .
\end{aligned}
$$

The third TL-moment is obtained in the same manner with relation (63) such as (with the previous notations):

$$
\begin{aligned}
\lambda_{3}^{(s, t)}= & 3^{-1} \xi_{\alpha}\left[\frac{(3+s+t) !}{t !} \frac{g(t+\xi)}{g(3+s+t+\xi)}\right. \\
& -2 \frac{(3+s+t) !}{(t+1) !} \frac{g(t+1+\xi)}{g(3+s+t+\xi)} \\
& \left.+\frac{(3+s+t) !}{(t+2) !} \frac{g(t+2+\xi)}{g(3+s+t+\xi)}\right] \\
= & \frac{(3+s+t) ! g(t+\xi)}{3 t ! g(3+s+t+\xi)} \xi_{\alpha}\left[1-2 \frac{h(t+\xi)}{(t+1)}\right. \\
& \left.+\frac{h(t+1+\xi) h(t+\xi)}{(t+2)(t+1)}\right] \\
= & \frac{(3+s+t) !}{3(t+2) !} \frac{g(t+\xi)}{g(3+s+t+\xi)} \alpha(1+\xi) .
\end{aligned}
$$

We finally end this appendix with the estimates of the parameters of a GPD, given by the Self-determined Probability-weighted Moment method, and we first begin by recalling hereafter the expression of the L-moments as a special case of the previous Proposition 11. 
Proposition 12 (see Hosking, 1990 and Abdul-Moniem and Selim, 2009). The first three L-moments, as a function of the three characteristic parameters of a $G P D$, are given by:

$$
\left\{\begin{array}{l}
\lambda_{1}=v+\alpha h(\xi) \\
\lambda_{2}=\alpha\{h(\xi)[1-h(\xi)]\}^{-1} \\
\lambda_{3}=g(\xi)[g(3+\xi)]^{-1} \alpha(1+\xi),
\end{array}\right.
$$

where $\lambda_{r}$ is the $r$-th $L$-moment with $r \in\{1,2,3\}, v \in \mathbb{R}$ the location parameter, $\alpha \in \mathbb{R}_{+}$the scale parameter, $\xi \in R^{*}$ the Shape-parameter and with $g()=$. $\Gamma[h()$.$] where h(k+j \xi)=(k+1-j \xi)$ for any $(j, k) \in\{1,2,3\} \times I N$, and $\Gamma(\xi)=\int_{0}^{+\infty} u^{\xi-1} e^{-u} d u$ the Gamma function.

Proof.

The proof is a direct consequence of Proposition 11 by taking $s=t=0$. The expressions of TL-moments of a GPD in some special cases of truncation order $(s, t)$ are also provided in Elamir and Seheult (2003), Hosking (2007) and Abdul-Moniem and Selim (2009). See Table 2-a for some specific results for the LH-moments of truncation order $(1,1)$ and the LL-moments of order $(1,1)$.

Proposition 13. The estimates $\hat{v}, \hat{\alpha}$ and $\hat{\xi}$, of $v \in \mathbb{R}$, the location parameter, $\alpha \in \mathbb{R}_{+}$, the scale parameter and $\xi \in \mathbb{R}^{*}$, the Shape-parameter, of a GPD, are obtained with the Self-determined Probability-weighted Moment method as a solution of the following system:

$$
\left\{\begin{aligned}
\hat{\xi}= & \left\{9\left[\hat{H}\left(\hat{X}_{[i: n]}\right)\right]^{2}-10 \hat{H}\left(\hat{X}_{[i: n]}\right)+2\right\} \\
& \times\left\{3\left[\hat{H}\left(\hat{X}_{[i: n]}\right)\right]^{2}-2 \hat{H}\left(\hat{X}_{[i: n]}\right)\right\}^{-1} \\
\hat{\alpha}= & n^{-1}(2+\hat{\xi}) h(\hat{\xi}) \\
& \times \sum_{i=1}^{n}\left\{\hat{X}_{[i: n]}\left[2 \hat{H}\left(\hat{X}_{[i: n]}\right)-1\right]\right\} \\
\hat{v}= & \bar{X}-\hat{\alpha}[h(\hat{\xi})]^{-1}
\end{aligned}\right.
$$

where $\left\{\hat{X}_{[i: n]}\right\}_{i \in[1, \ldots, n]}$ are the empirical order statistics of Log-volatility exceedances, $\hat{H}\left(\hat{X}_{[i: n]}\right)=1-\left\{1+\hat{\xi}_{\hat{\alpha}}^{-1}\left\{\hat{X}_{[i: n]}-\bar{X}+\hat{\alpha}[h(\hat{\xi})]^{-1}\right\}\right\}^{-1 / \hat{\xi}}$ is the empirical estimate of a GPD CDF of estimated order statistics, and with $\bar{X}=$ $n^{-1} \sum_{i=1}^{n} \hat{X}_{[i: n]}, \hat{\xi}_{\hat{\alpha}}=\hat{\alpha} / \hat{\xi}$, and $h(k+j \hat{\xi})=(k+1-j \hat{\xi})$ for any $(j, k) \in$ $\{1,2,3\} \times I N$.

Proof. 
Using the expression of the GPD parameters with L-moments in Proposition 12 and the relation between L-moments and PwM recalled in Proposition 1, we get (with the previous notations):

$$
\left\{\begin{array}{l}
\xi=\left[9 \beta_{2}-10 \beta_{1}+2 \beta_{0}\right]\left(3 \beta_{2}-2 \beta_{1}\right)^{-1} \\
\alpha=\left[2 \beta_{1}-\beta_{0}\right](2+\xi) h(\xi) \\
v=\beta_{0}-\alpha[h(\xi)]^{-1}
\end{array}\right.
$$

In the special case of the Sd-PwM method, the estimate of the $\hat{\beta}_{j}$ are directly defined such as (with the previous notations):

$$
\hat{\beta}_{j}=n^{-1} \sum_{i=1}^{n}\left[\hat{X}_{[i: n]} \hat{H}\left(\hat{X}_{[i: n]}\right)\right],
$$

where $\hat{H}($.$) is a GPD CDF estimate such as (with the previous notations):$

$$
\hat{H}\left(\hat{X}_{[i: n]}\right)=1-\left\{1+\hat{\xi}_{\hat{\alpha}}^{-1}\left\{\hat{X}_{[i: n]}-\bar{X}+\hat{\alpha}[h(\hat{\xi})]^{-1}\right\}\right\}^{-1 / \hat{\xi}} .
$$

This leads, from equations (100) with estimated parameters, to the desired result.

Solving the system eventually allows us to recover from the data the value of the parameter $\hat{\xi}$, altogether with $\hat{\alpha}$ and $\hat{v}$. 


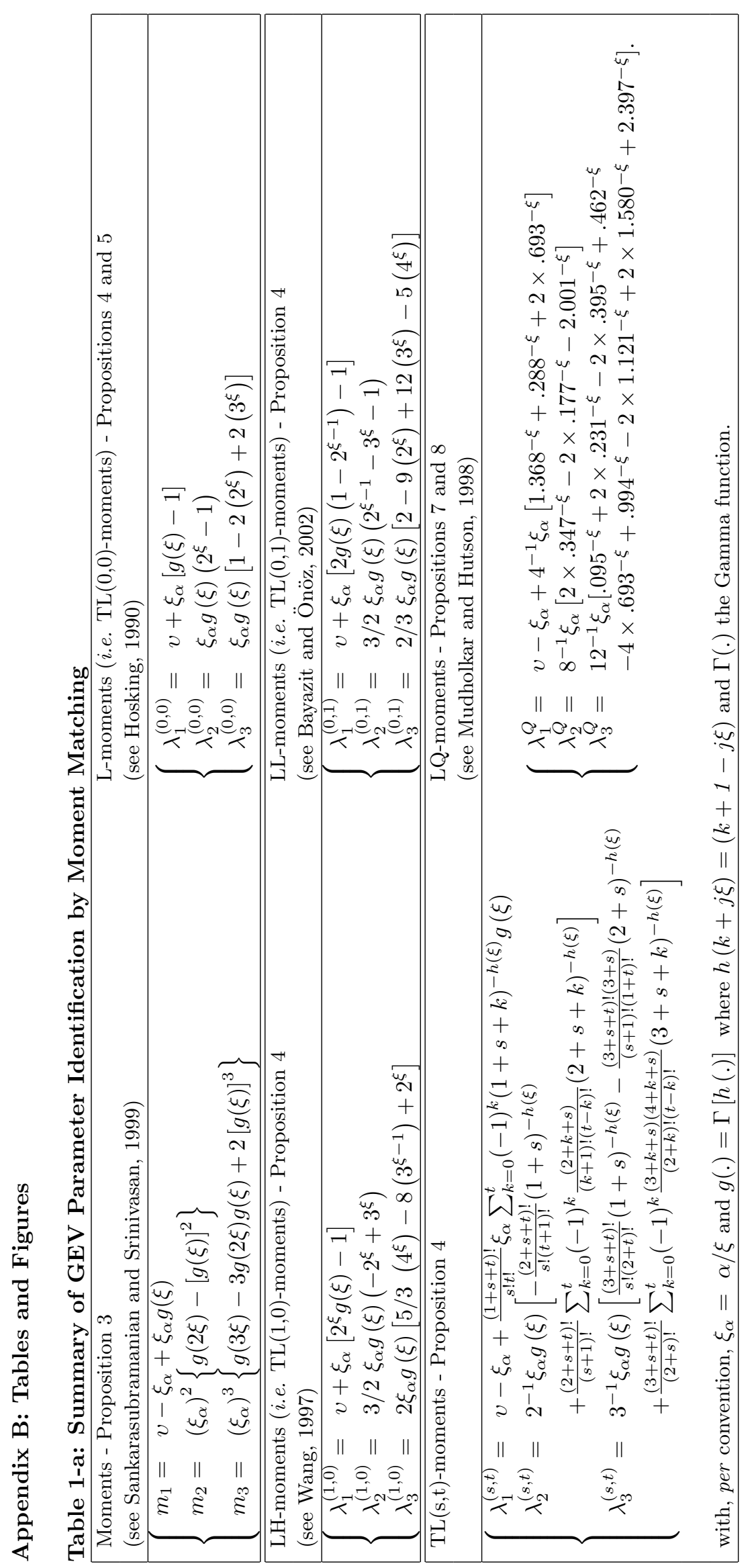


Table 1-b: GEV Parameter Identification with the Self-determined Probability-weighted Moment Method

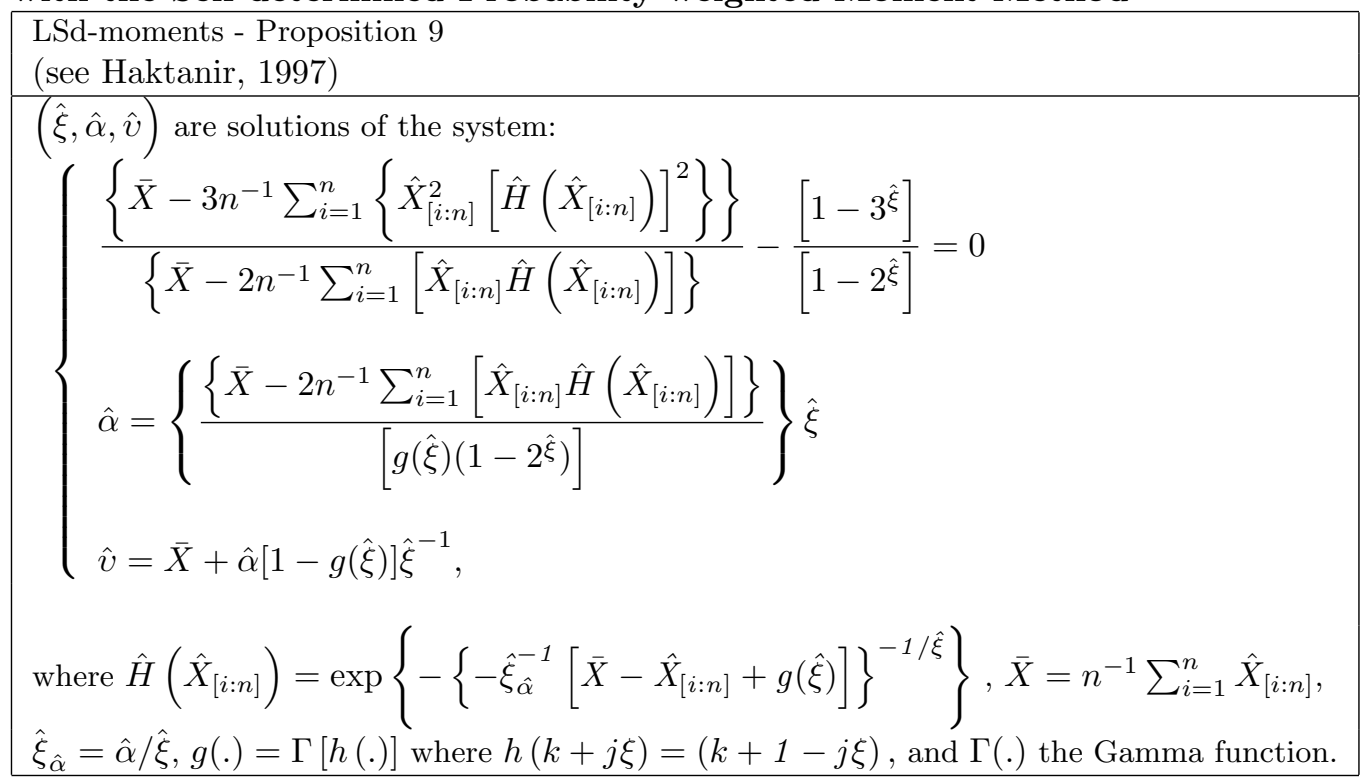




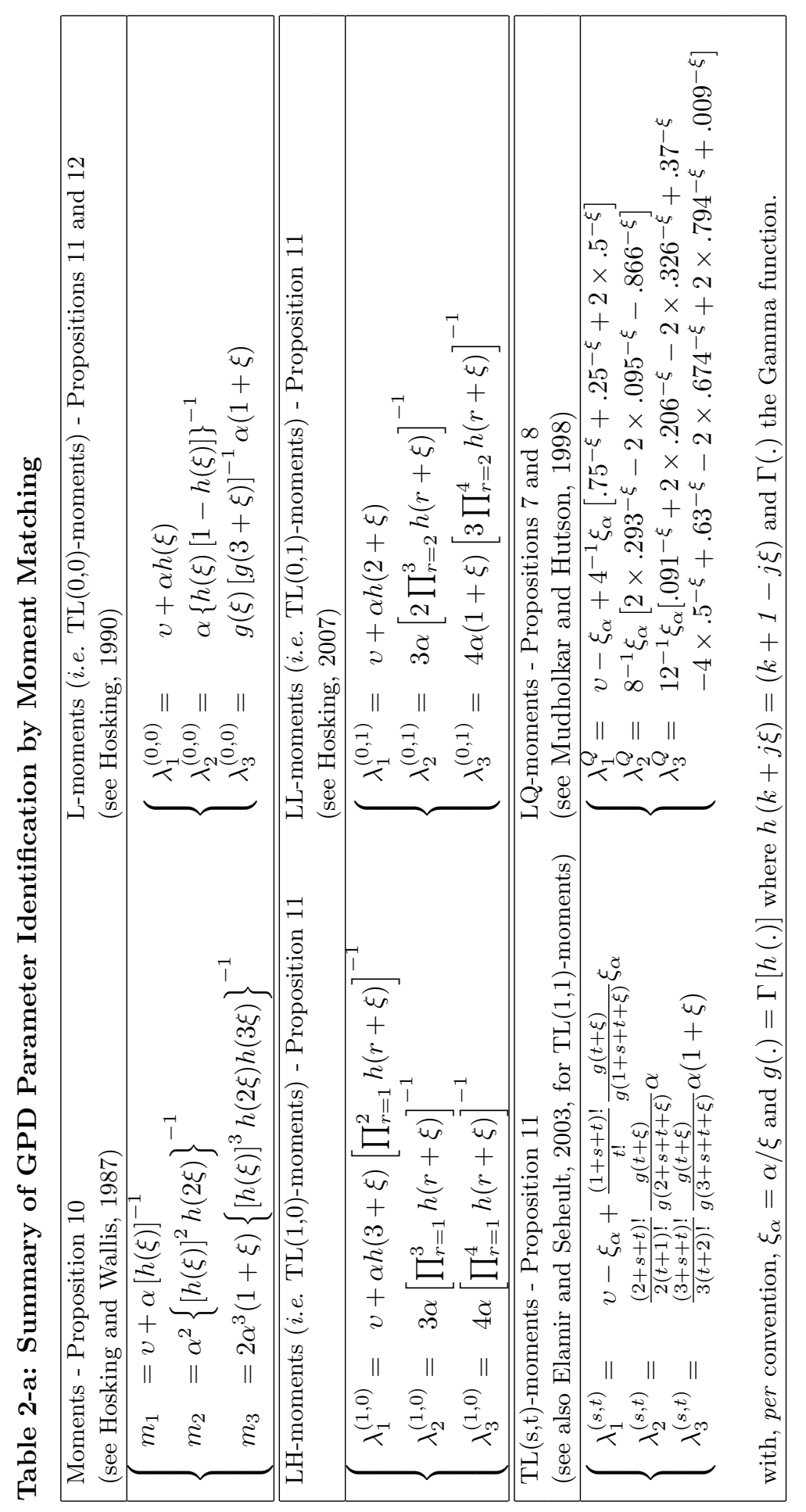


Table 2-b: GPD Parameter Identification with the Self-determined Probability-weighted Moment Method

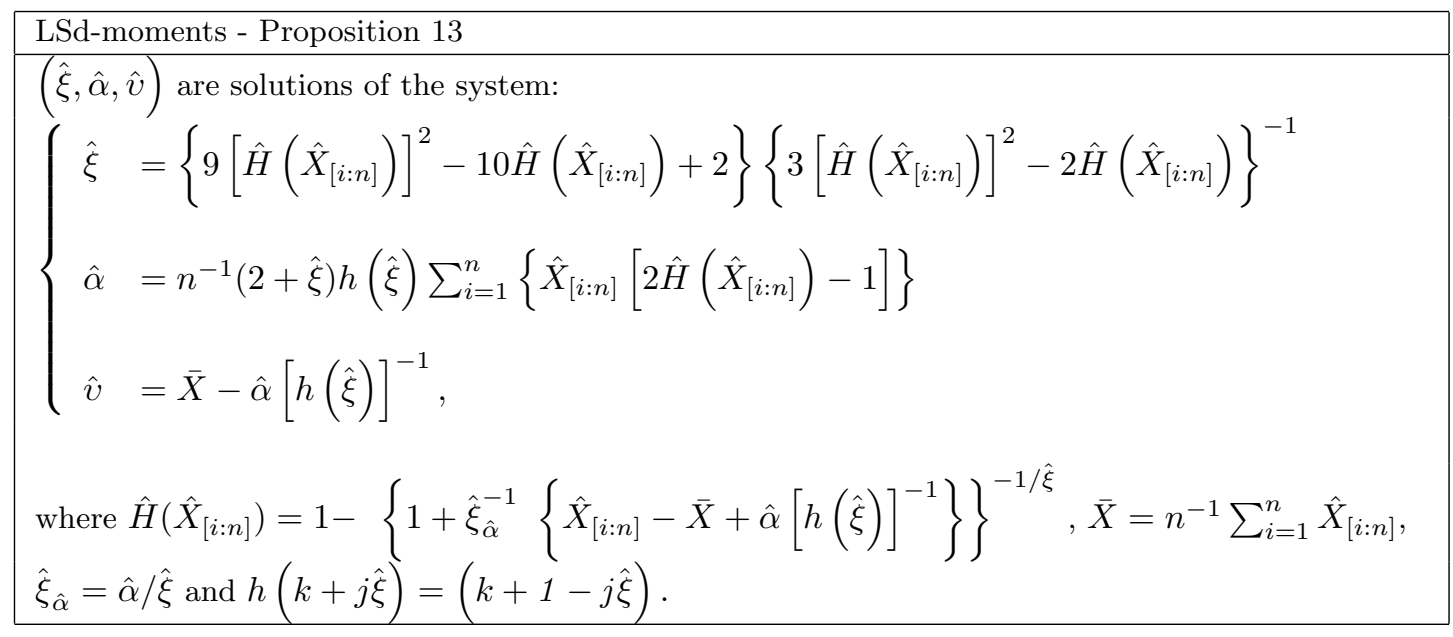


Figure 1-a: Daily Estimates of Annualized Volatilities
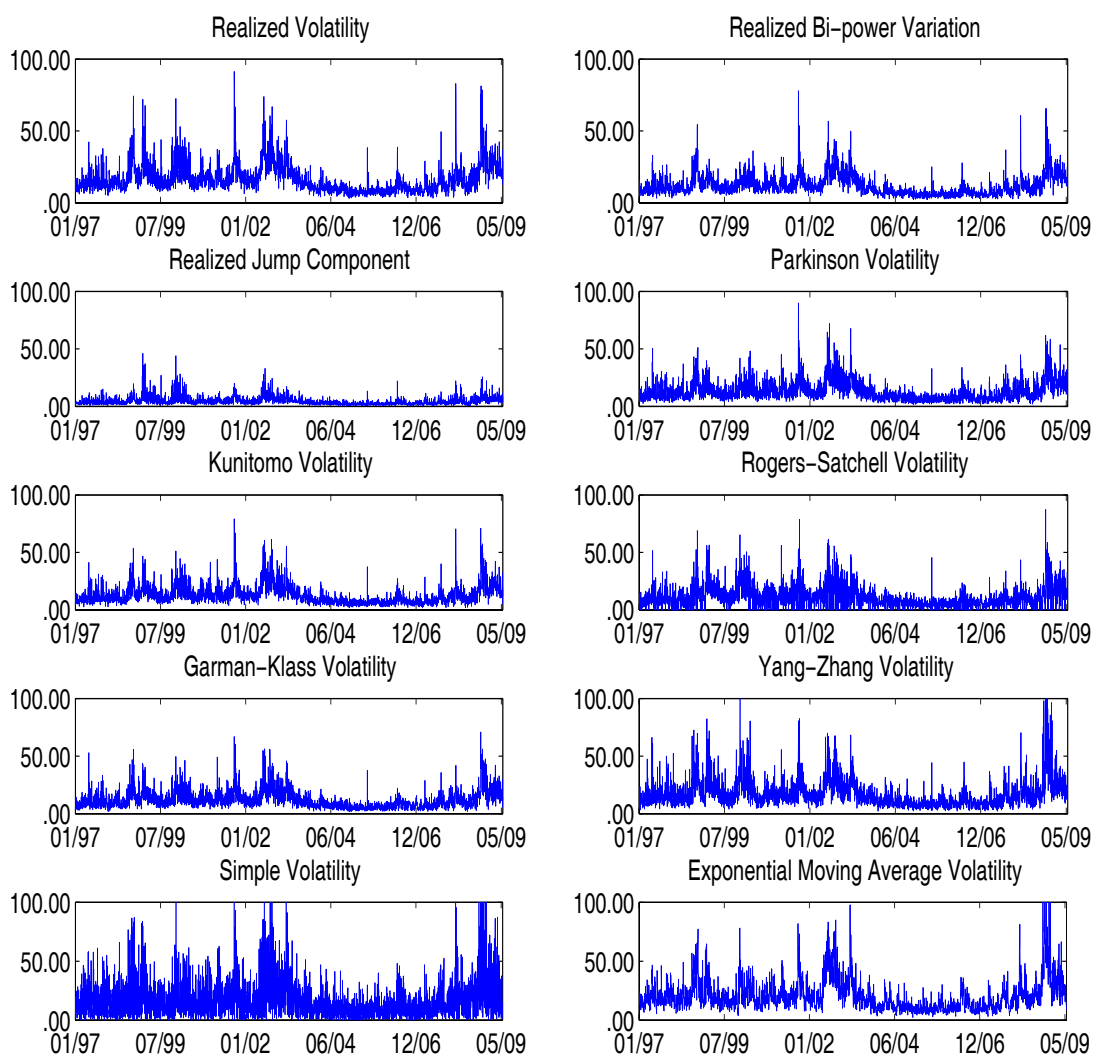

Source: Euronext, 30' resampled intraday CAC40 French stock index quotes from the period 01-01-1997/05-31-2009. Volatility estimations are annualized here and expressed in percentage (on the y-axis). Computations by the authors. 
Figure 1-b: Probability-to-Probability Plot

of Empirical versus Normal and GEV

of Daily Estimates of Annualized Log-volatilities
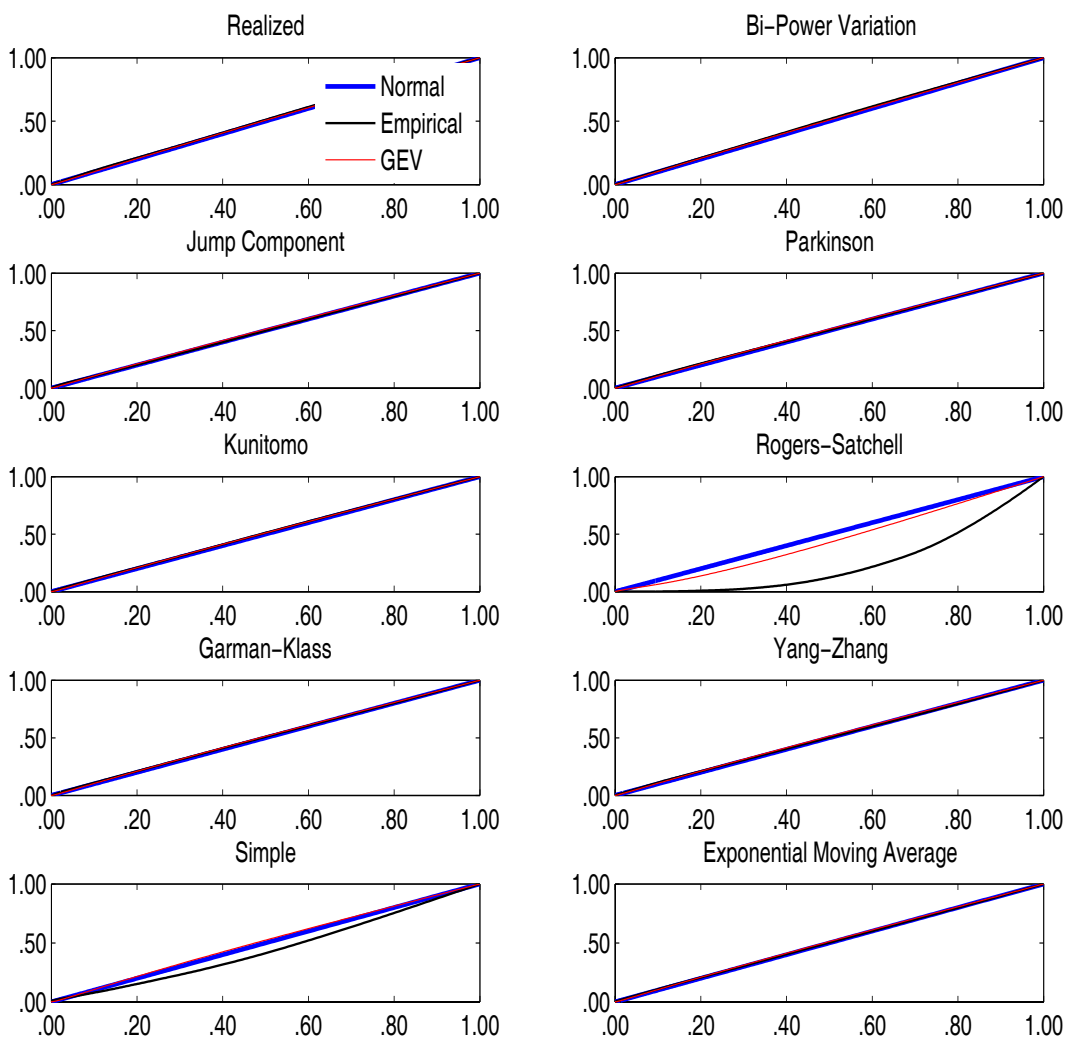

Source: Euronext, 30' resampled intraday CAC40 French stock index quotes from the period 01-01-1997/05-31-2009. Log-volatility estimations (on the x-axis) are annualized and expressed in percentage. The Empirical Gaussian Kernel Density estimates (on the y-axis) are obtained using the cross-validation principle (Silverman, 1986). Computations by the authors. 
Figure 1-c: A Zoom on Extreme Values of the Densities of Daily Estimates of Annualized Volatilities
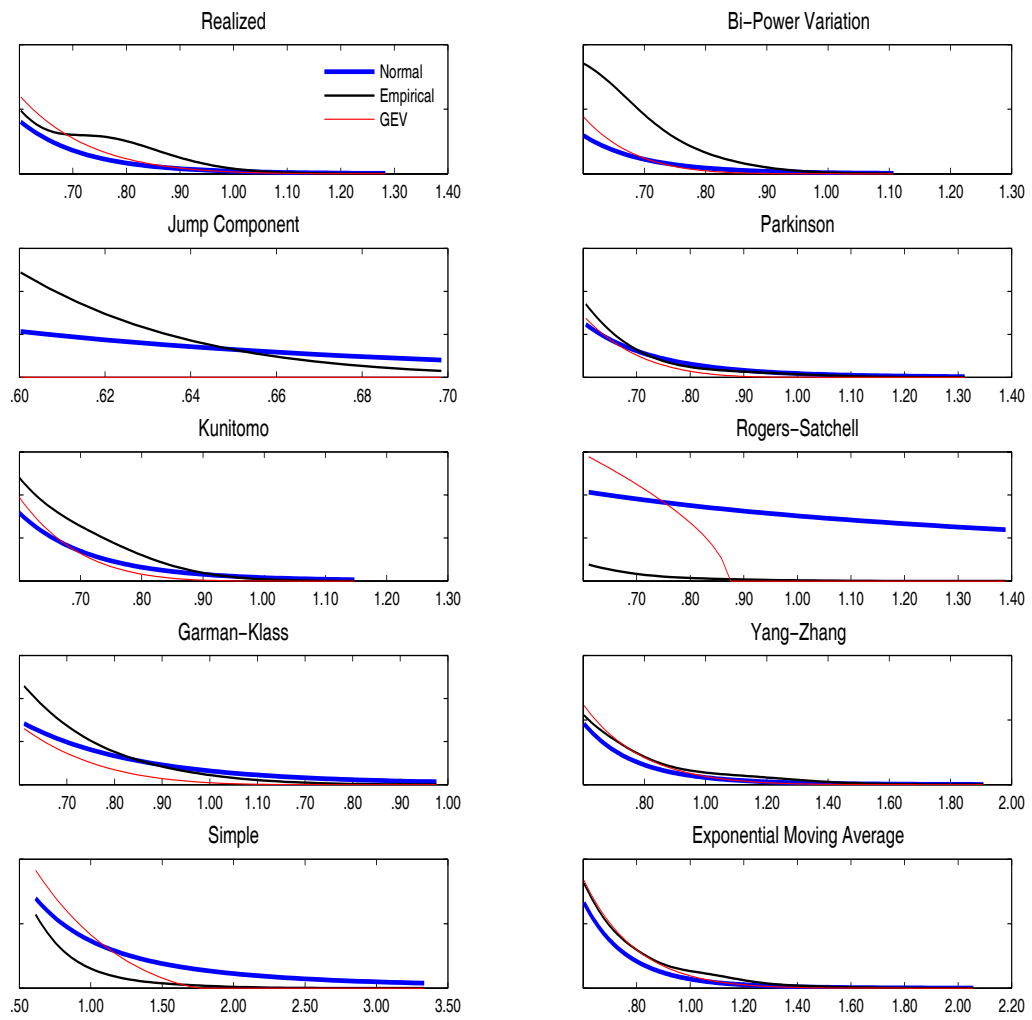

Source: Euronext, 30' resampled intraday CAC40 French stock index quotes from the period 01-01-1997/05-31-2009. Volatility estimations (on the x-axis) are annualized and expressed in percentage. The Empirical Gaussian Kernel Density estimates (on the y-axis) are obtained using the cross-validation principle (Silverman, 1986). Computations by the authors. 
Figure 1-d: Empirical Densities of Estimated Extremal Indexes of Weekly Extreme Annualized Daily Volatilities
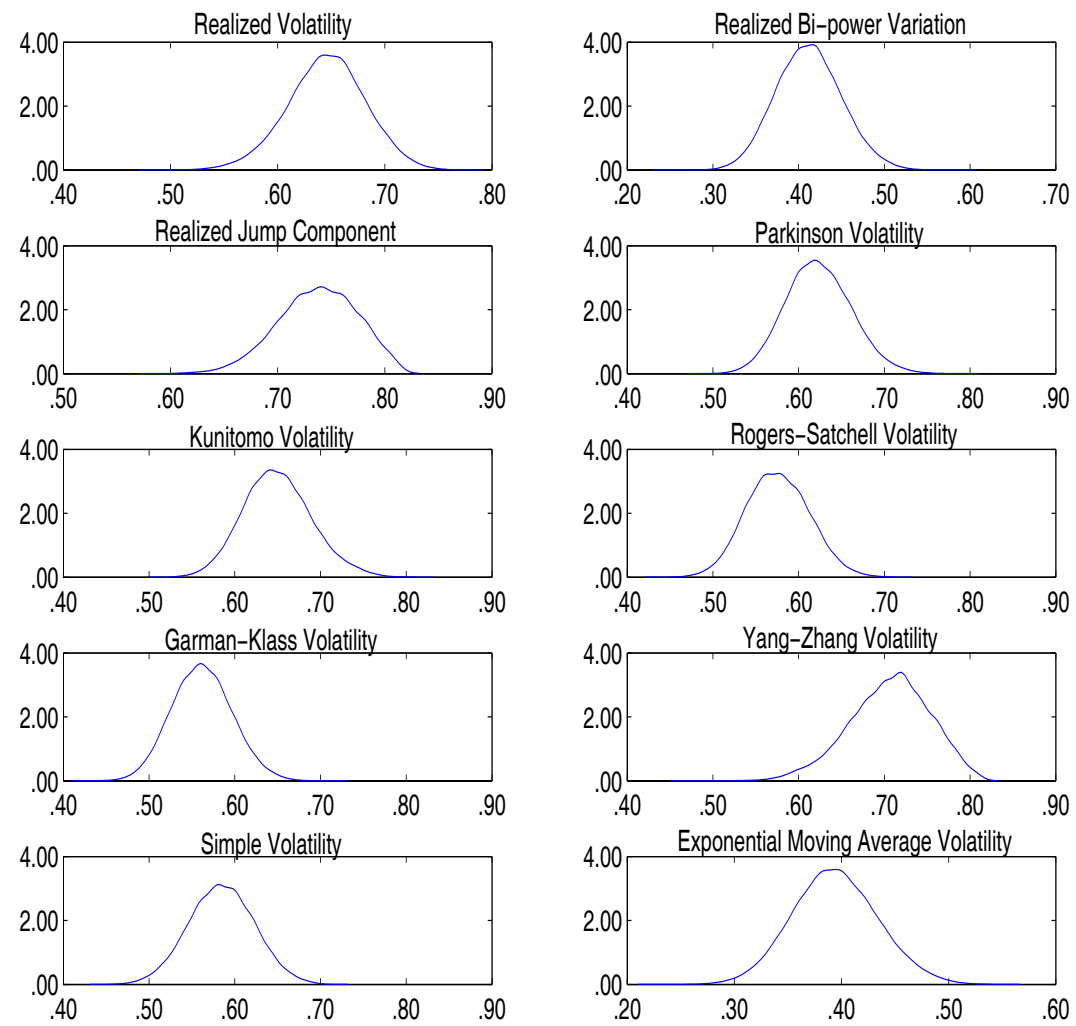

Source: Euronext, 30' resampled intraday CAC40 French stock index quotes from the period 01-01-1997/05-31-2009. Extremal Indexes (on the x-axis) are computed according to the formula in equation (31), for $n=5$ (weekly blocks), $m=500$ (number of blocks) and a threshold fixed at $99.00 \%$. The Empirical Gaussian Kernel Density estimates of 10,000 bootstrapped samples (on the y-axis) are obtained using the cross-validation principle (Silverman, 1986). Computations by the authors. 
Figure 2: Beirlant et al. (1996) Non-parametric Generalized Hill Estimates of Extreme Value Shape-parameters of Volatility Densities

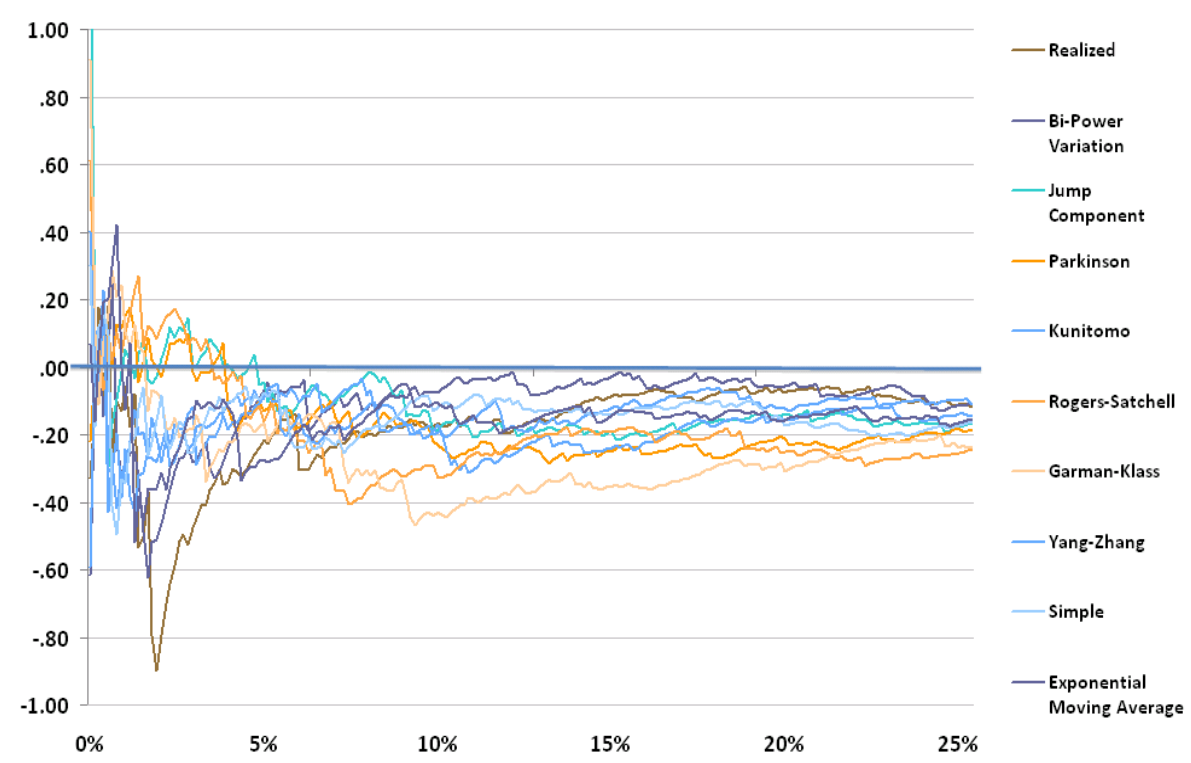

Source: Euronext, 30' resampled intraday CAC40 French stock index quotes from the period 01-01-1997/05-31-2009. The value of the estimates (on the y-axis) is plotted against the quantiles of data points included in the sample of extreme observations (on the x-axis). Computations by the authors. 
Figure 3: Empirical Kernel Estimates

of Cumulative Distribution Functions of Shape-parameters and Characteristics

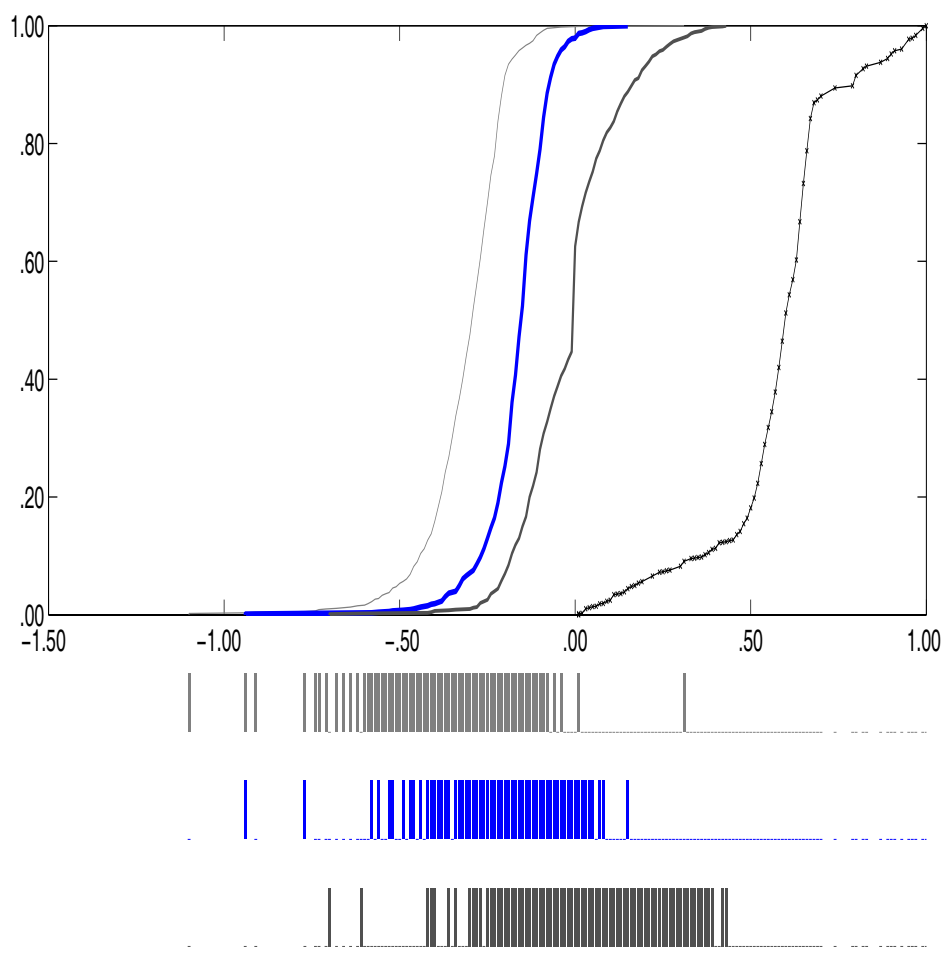

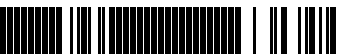

Source: Euronext, 30' resampled intraday CAC40 French stock index quotes from the period 01-01-1997/05-31-2009. Empirical Gaussian Kernel estimates using the cross-validation principle (Silverman, 1986). In the first figure (at the top), the main empirical rectangular kernel CDF (on the y-axis) (second from the left) corresponds to the 1,440 Shape-parameters estimated according to the several methodologies used (see text), whilst the two to the immediate left and right correspond to the distributions of their related thresholds fixed at $5 \%$ and $95 \%$. The final right empirical CDF (on the y-axis) is relative to the density of the Kolmogorov-Smirnov Goodness-of-Fit P-statistic (between .00 and 1.00 on the x-axis) of implied extreme value densities with estimated parameters. The three lower figures (at the bottom) respectively represent all the occurences of the $5 \%$ minima, mean Shape-parameters, 95\% maxima and Kolmogorov-Smirnov P-statistics. Computations by the authors. 


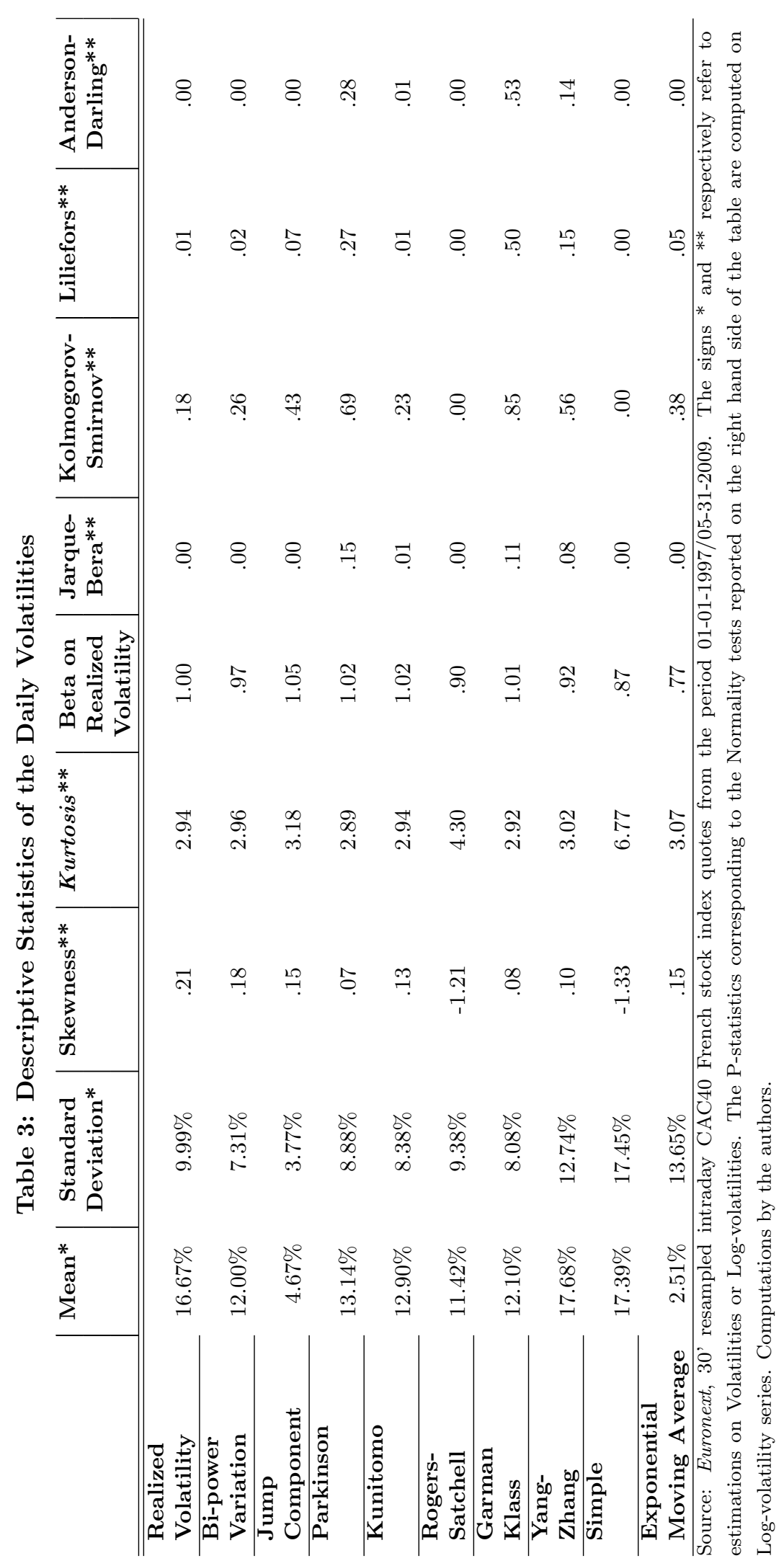




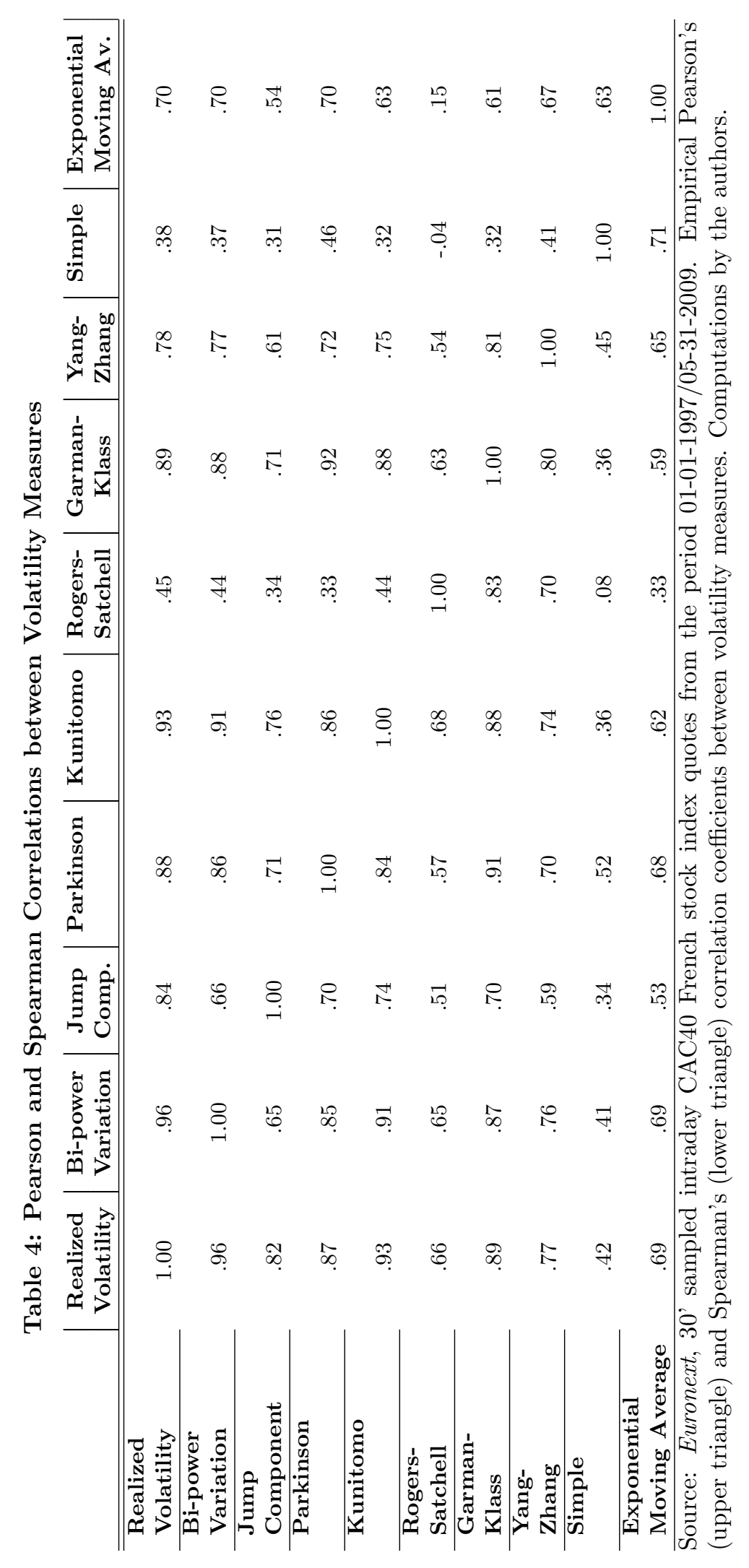




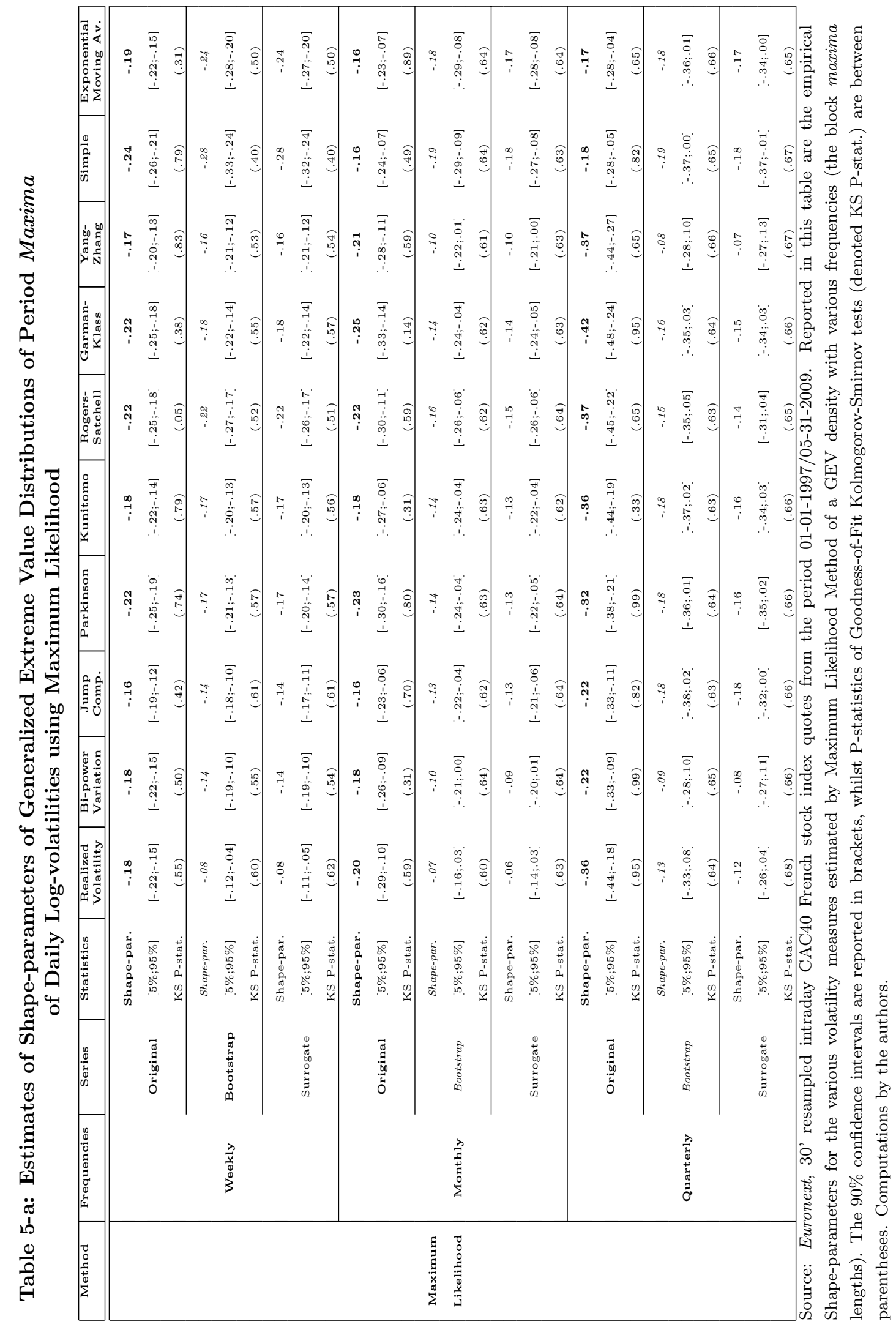




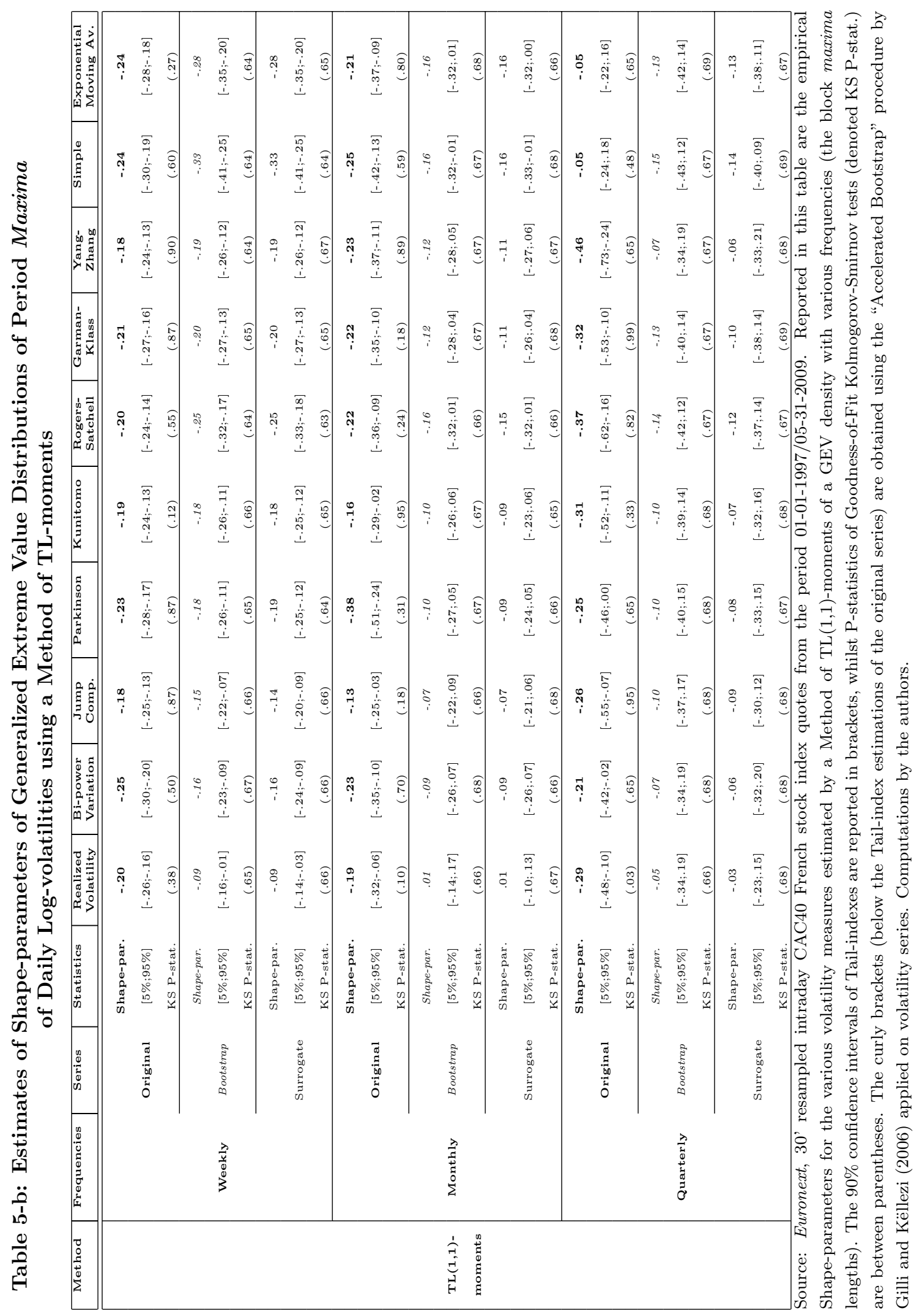




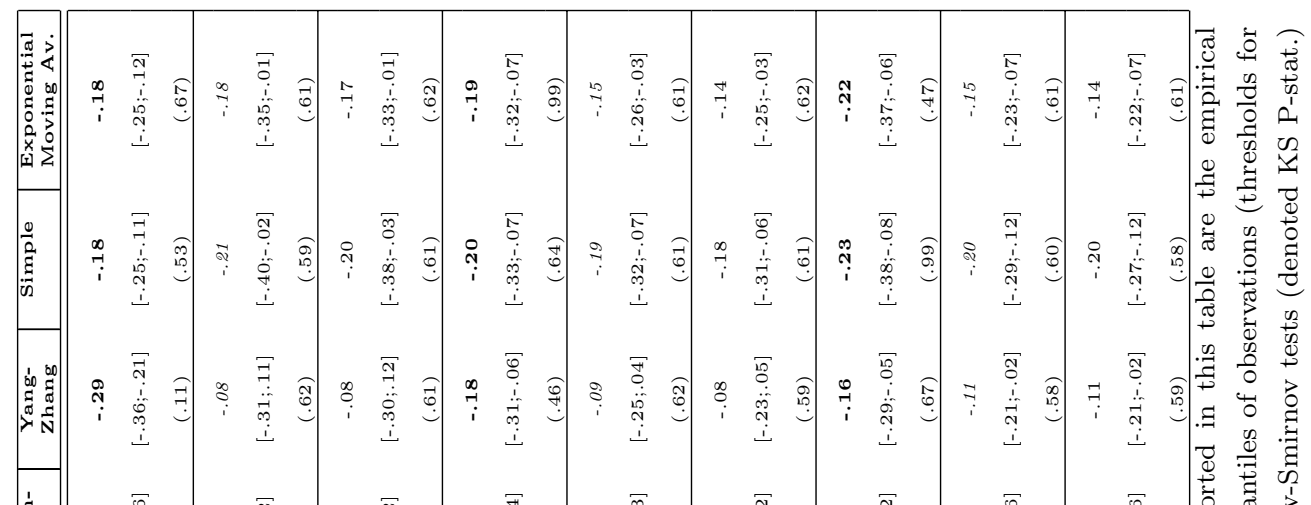

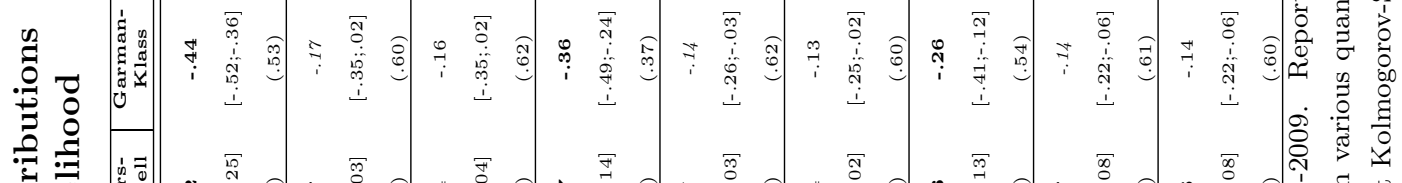

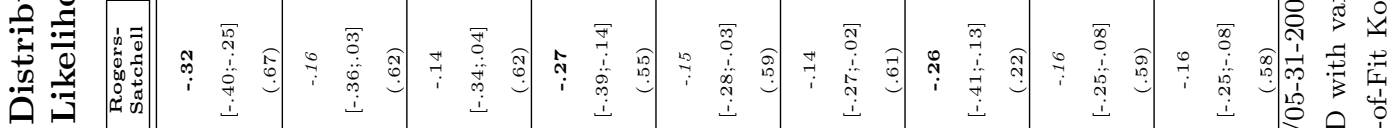

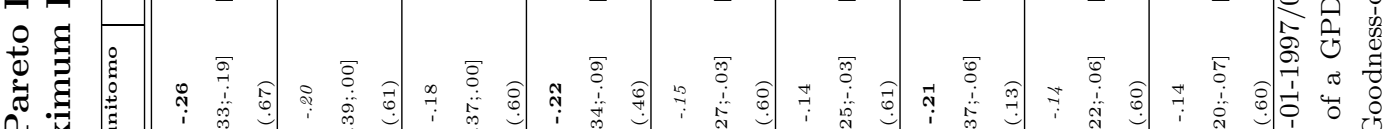

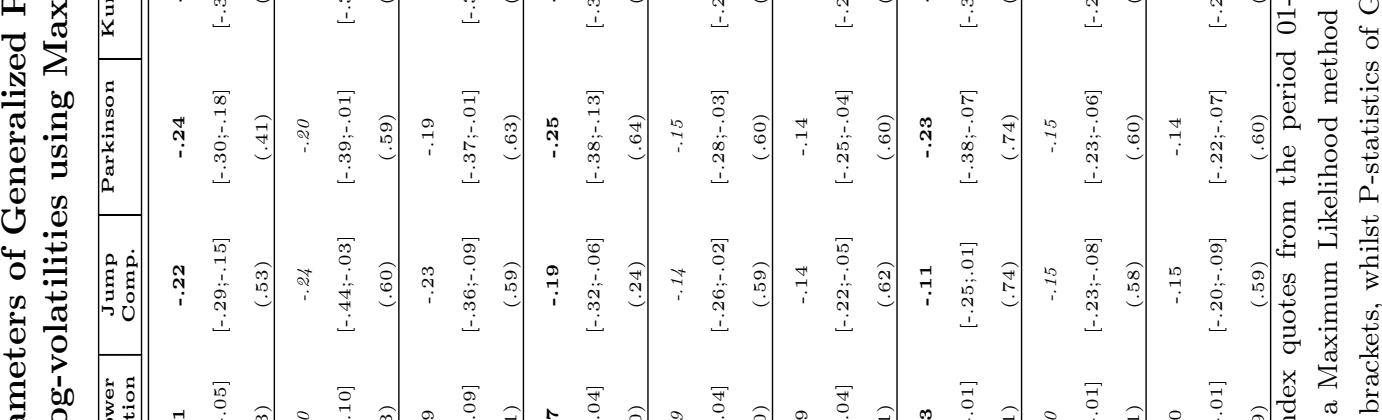

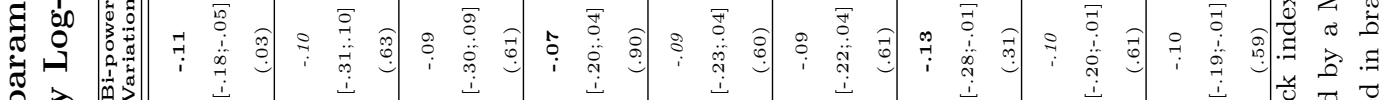

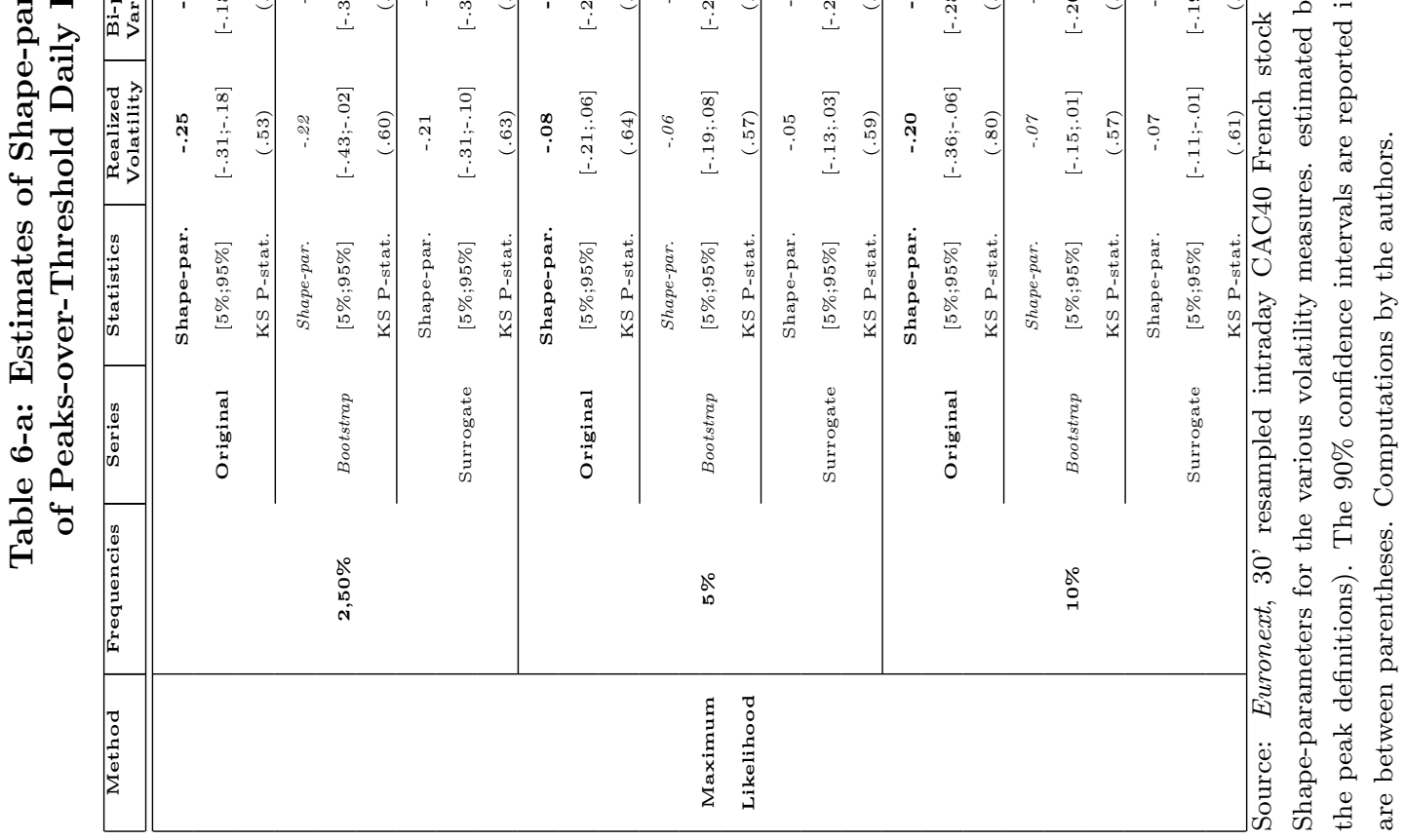




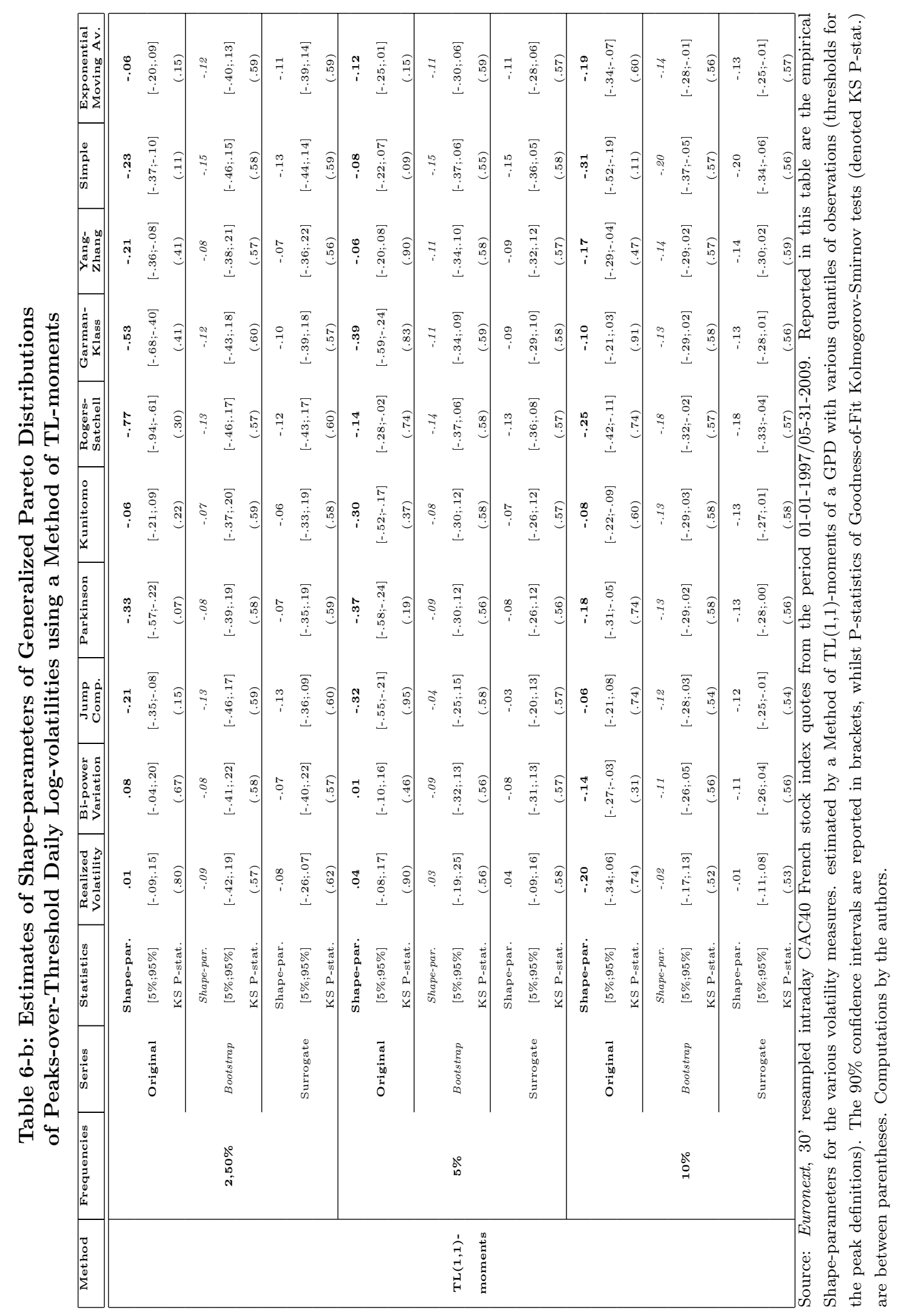


Table 7: Probablity of the Largest Negative Returns, the Largest Daily Volatilities and Return-times using Maximum Likelihood GEV Estimates

\begin{tabular}{|c|c|c|c|c|c|c|c|c|}
\hline \multirow[b]{2}{*}{ Estimators } & \multirow[b]{2}{*}{ Crisis } & \multirow[b]{2}{*}{ Values } & \multicolumn{2}{|c|}{ Weekly } & \multicolumn{2}{|c|}{ Monthly } & \multicolumn{2}{|c|}{ Quarterly } \\
\hline & & & Prob. & $\begin{array}{c}\text { Return } \\
\text { Times }\end{array}$ & Prob. & $\begin{array}{c}\text { Return } \\
\text { Times }\end{array}$ & Prob. & $\begin{array}{c}\text { Return } \\
\text { Times }\end{array}$ \\
\hline \multirow{3}{*}{ Returns } & $10 / 06 / 2008$ & $-9.47 \%$ & $.14 \%$ & $14.31^{*}$ & $.65 \%$ & 12.40 & $1.92 \%$ & $13.56^{*}$ \\
\hline & $10 / 10 / 2008$ & $-8.05 \%$ & $.33 \%$ & 6.14 & $1.27 \%$ & 6.28 & $3.63 \%$ & 7.17 \\
\hline & $09 / 11 / 2001$ & $-7.48 \%$ & $.47 \%$ & 4.30 & $1.70 \%$ & 4.69 & $4.76 \%$ & 5.46 \\
\hline \multirow{4}{*}{ Realized } & $09 / 11 / 2001$ & .91 & $.22 \%$ & 9.16 & $.97 \%$ & 8.23 & $1.66 \%$ & $15.69^{*}$ \\
\hline & $01 / 21 / 2008$ & .83 & $.40 \%$ & 5.05 & $1.53 \%$ & 5.22 & $3.30 \%$ & 7.88 \\
\hline & $10 / 10 / 2008$ & .81 & $.45 \%$ & 4.48 & $1.68 \%$ & 4.77 & $3.74 \%$ & 6.95 \\
\hline & $09 / 11 / 2001$ & .78 & $.06 \%$ & $31.31^{*}$ & $.50 \%$ & $16.10^{*}$ & $1.37 \%$ & $18.93^{*}$ \\
\hline Bi-Power & $10 / 10 / 2008$ & .66 & $.22 \%$ & 8.98 & $1.10 \%$ & 7.25 & $3.15 \%$ & 8.26 \\
\hline \multirow[t]{2}{*}{ Variation } & $10 / 16 / 2008$ & .65 & $.23 \%$ & 8.60 & $1.14 \%$ & 7.04 & $3.24 \%$ & 8.02 \\
\hline & $01 / 15 / 1999$ & .46 & $.12 \%$ & $16.66^{*}$ & $.60 \%$ & $13.35^{*}$ & $1.24 \%$ & $21.03^{*}$ \\
\hline Jump & $01 / 04 / 2000$ & .44 & $.16 \%$ & $12.87^{*}$ & $.73 \%$ & 10.92 & $1.57 \%$ & $16.58^{*}$ \\
\hline \multirow[t]{2}{*}{ Component } & 02/09/1999 & .37 & $.36 \%$ & 5.61 & $1.41 \%$ & 5.65 & $3.30 \%$ & 7.87 \\
\hline & $09 / 11 / 2001$ & .90 & $.03 \%$ & $58.69^{*}$ & $.26 \%$ & $30.39^{*}$ & $.59 \%$ & $43.76^{*}$ \\
\hline \multirow[t]{3}{*}{ Parkinson } & $08 / 06 / 2002$ & .72 & $.27 \%$ & 7.33 & $1.14 \%$ & 7.01 & $3.35 \%$ & 7.77 \\
\hline & $03 / 17 / 2003$ & .68 & $.42 \%$ & 4.71 & $1.60 \%$ & 5.00 & $4.73 \%$ & 5.50 \\
\hline & $09 / 11 / 2001$ & .79 & $.20 \%$ & 10.09 & $.81 \%$ & 9.93 & $1.40 \%$ & $18.54^{*}$ \\
\hline \multirow[t]{3}{*}{ Kunitomo } & $10 / 08 / 2008$ & .71 & $.39 \%$ & 5.16 & $1.40 \%$ & 5.72 & $3.23 \%$ & 8.05 \\
\hline & $01 / 21 / 2008$ & .70 & $.40 \%$ & 4.99 & $1.44 \%$ & 5.57 & $3.35 \%$ & 7.76 \\
\hline & $10 / 08 / 2008$ & .87 & $.10 \%$ & $19.22^{*}$ & $.79 \%$ & 10.08 & $.98 \%$ & $26.64^{*}$ \\
\hline Rogers- & $09 / 21 / 2001$ & .79 & $.23 \%$ & 8.58 & $1.30 \%$ & 6.15 & $2.32 \%$ & 11.19 \\
\hline \multirow[t]{2}{*}{ Satchell } & $10 / 02 / 1998$ & .69 & $.55 \%$ & 3.66 & $2.29 \%$ & 3.49 & $5.16 \%$ & 5.04 \\
\hline & $10 / 08 / 2008$ & .71 & $.18 \%$ & 10.93 & $.78 \%$ & 10.24 & $1.10 \%$ & $23.70^{*}$ \\
\hline Garman- & $09 / 11 / 2001$ & .67 & $.28 \%$ & 7.02 & $1.11 \%$ & 7.18 & $2.06 \%$ & $12.61^{*}$ \\
\hline \multirow[t]{2}{*}{ Klass } & $09 / 11 / 2001$ & .60 & $.59 \%$ & 3.40 & $2.01 \%$ & 3.97 & $4.82 \%$ & 5.39 \\
\hline & $10 / 24 / 2008$ & 1.29 & $.19 \%$ & 10.36 & $.56 \%$ & $14.20^{*}$ & $.64 \%$ & $40.69^{*}$ \\
\hline Yang- & $10 / 13 / 2008$ & 1.21 & $.27 \%$ & 7.39 & $.78 \%$ & 10.24 & $1.20 \%$ & $21.62^{*}$ \\
\hline \multirow[t]{2}{*}{ Zhang } & $10 / 10 / 2008$ & 1.14 & $.37 \%$ & 5.43 & $1.05 \%$ & 7.62 & $1.97 \%$ & $13.21^{*}$ \\
\hline & $10 / 13 / 2008$ & 1.77 & $.03 \%$ & $62.08^{*}$ & $.34 \%$ & $23.64^{*}$ & $1.22 \%$ & $21.34^{*}$ \\
\hline \multirow[t]{3}{*}{ Simple } & $11 / 24 / 2008$ & 1.60 & $.10 \%$ & 20.45 & $.60 \%$ & $13.24^{*}$ & $1.99 \%$ & $13.04^{*}$ \\
\hline & $09 / 19 / 2008$ & 1.47 & $.20 \%$ & 9.76 & $.94 \%$ & 8.51 & $2.91 \%$ & 8.93 \\
\hline & $10 / 13 / 2008$ & 1.43 & $.07 \%$ & $30.18^{*}$ & $.29 \%$ & $27.90^{*}$ & $1.10 \%$ & $23.61^{*}$ \\
\hline Exponential & $11 / 24 / 2008$ & 1.19 & $.24 \%$ & 8.26 & $.78 \%$ & 10.31 & $2.55 \%$ & 10.19 \\
\hline Moving Average & $10 / 29 / 2008$ & 1.08 & $.43 \%$ & 4.65 & $1.24 \%$ & 6.48 & $3.79 \%$ & 6.86 \\
\hline
\end{tabular}




\section{APPENDIX C \\ "Extreme Volatilities, Financial Crises \\ and L-moment Estimations of Tail-indexes"}

- April 2010 -

\section{- Complementary Results for the Referees' Attention - Available upon Demand for Readers}

In this appendix, which can be mailed to Readers by request to the Authors, we give additional results that were mentioned and explained in the main paper but not presented due to the length constraint of the publication. The paper is self-sufficient, but this appendix provides more detailed results. 
$N$

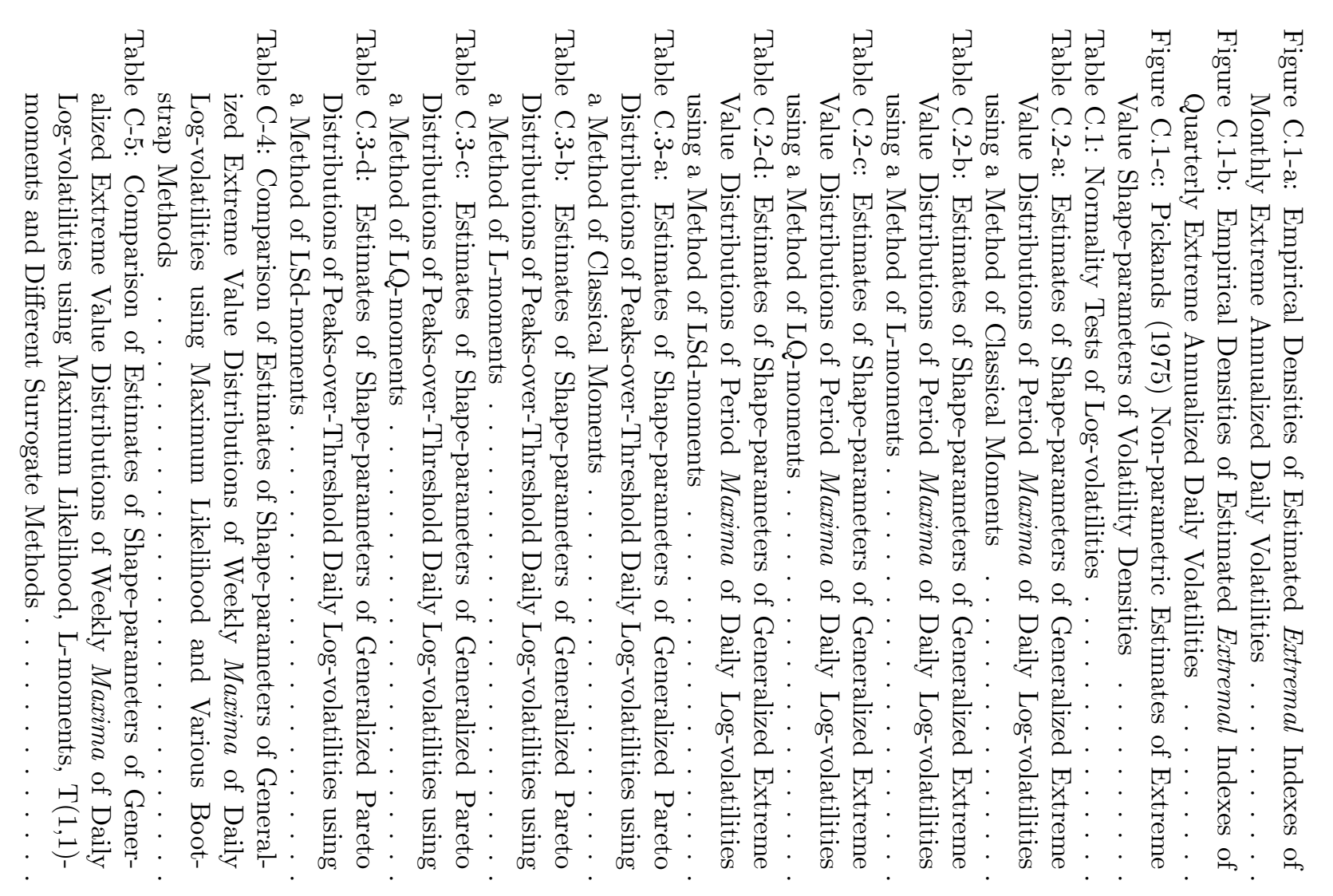

$\frac{8}{8}$

$\checkmark$

5

它占

co 


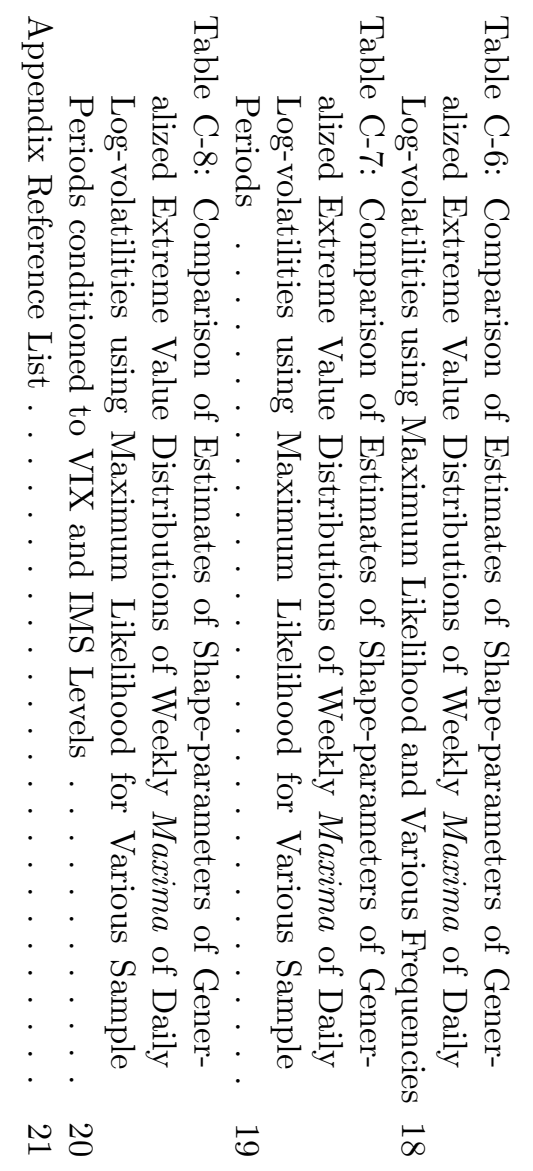



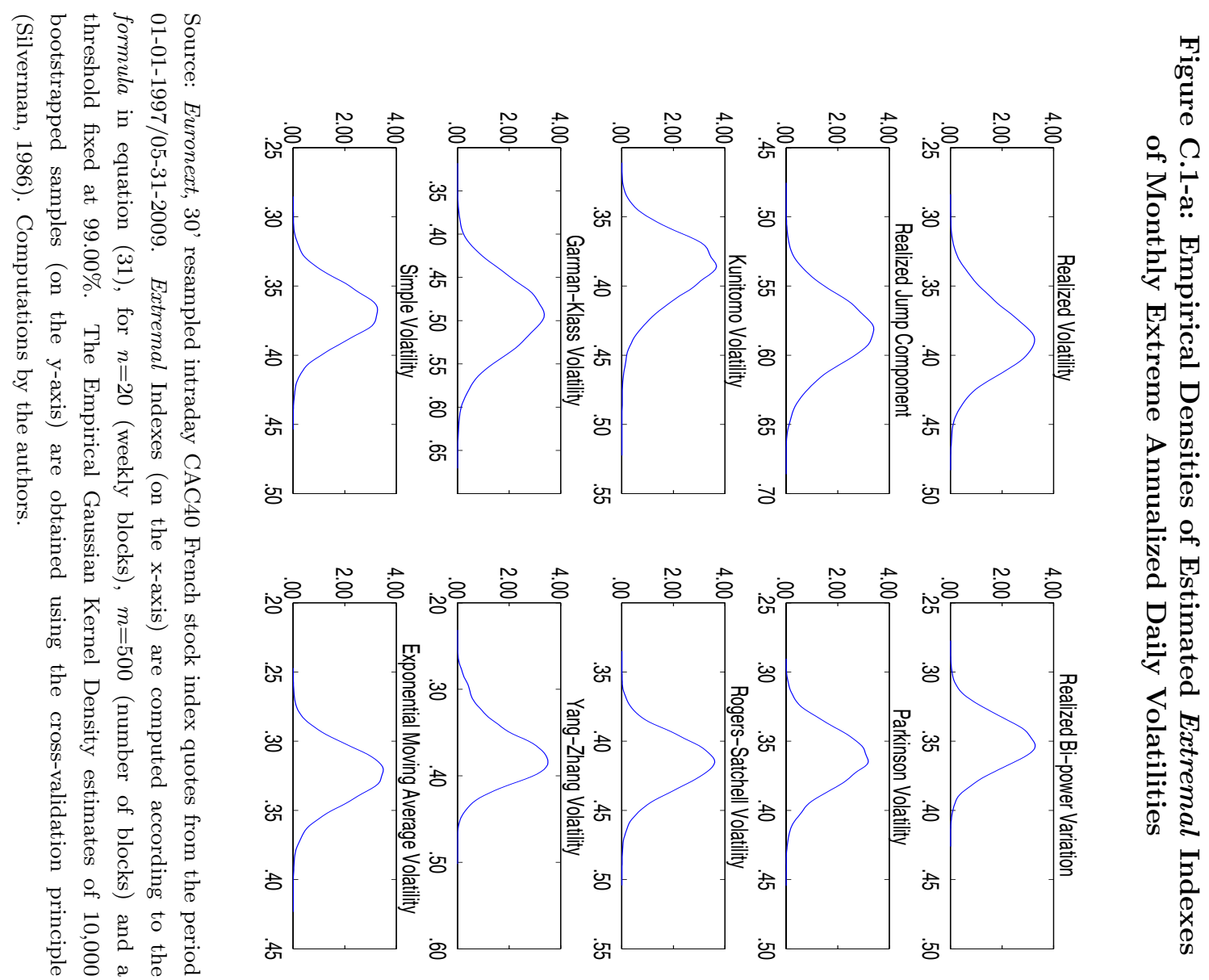

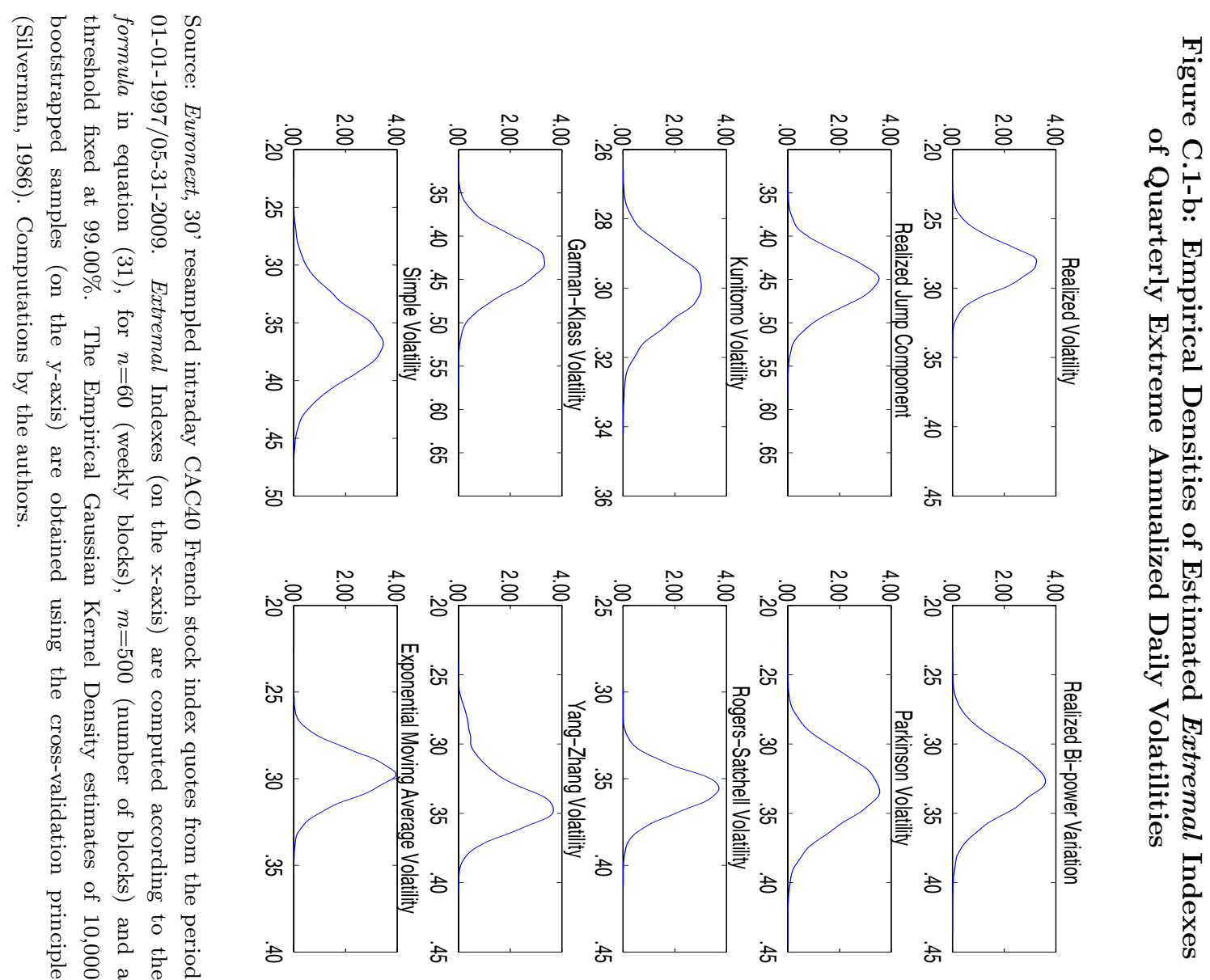


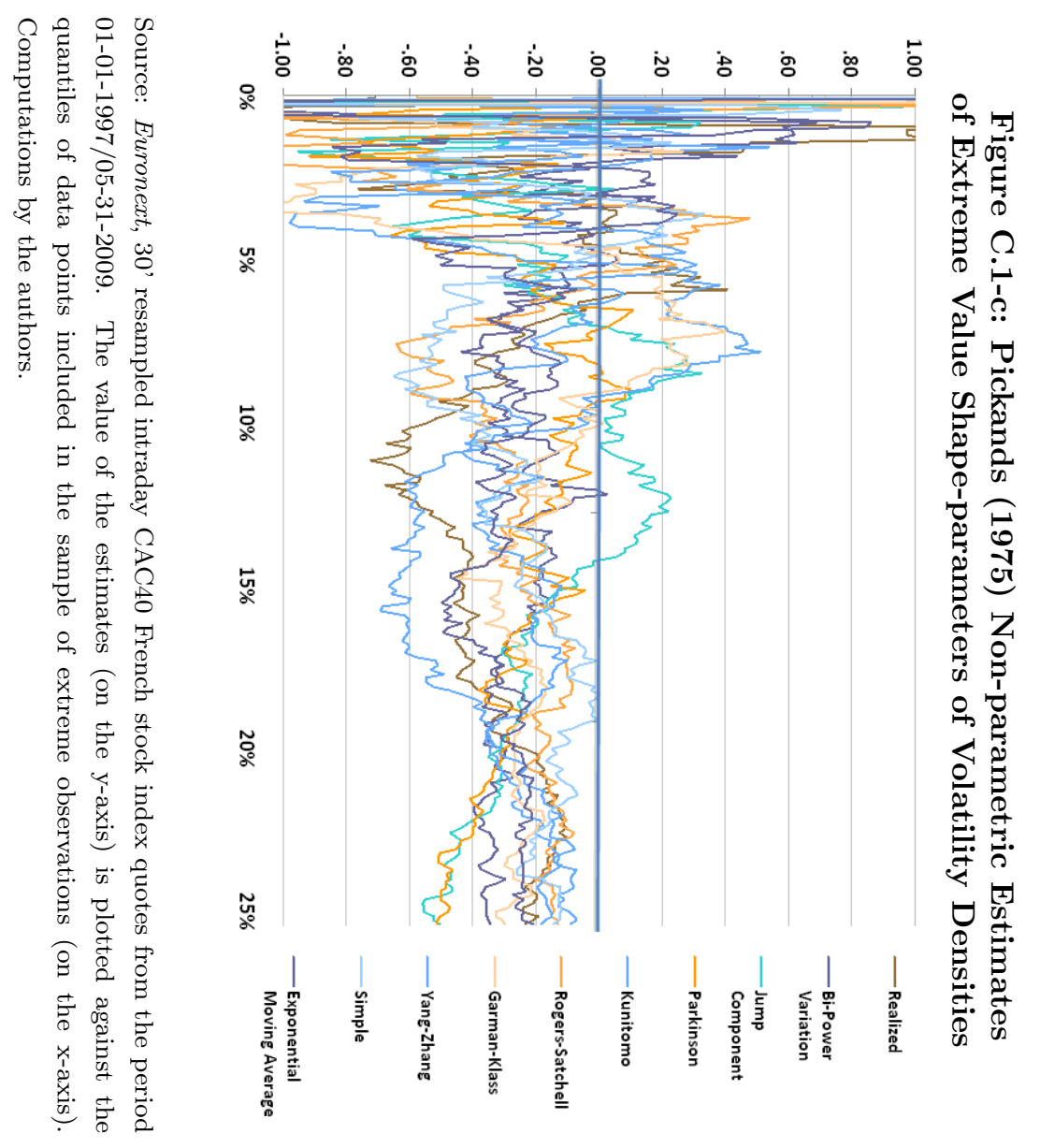


Table C.1: Normality Tests of Log-volatilities

\begin{tabular}{|c|c|c|c|c|c|c|c|c|c|c|c|}
\hline Series & $\begin{array}{l}\text { Normality } \\
\text { Tests } \\
\end{array}$ & $\begin{array}{l}\text { Realized } \\
\text { Volatility } \\
\end{array}$ & $\begin{array}{l}\text { Bi-power } \\
\text { Variation } \\
\end{array}$ & $\begin{array}{l}\text { Jump } \\
\text { Comp. }\end{array}$ & Parkinson & Kunitomo & $\begin{array}{l}\text { Rogers- } \\
\text { Satchell } \\
\end{array}$ & $\begin{array}{l}\text { Garman- } \\
\text { Klass } \\
\end{array}$ & $\begin{array}{l}\text { Yang- } \\
\text { Zhang } \\
\end{array}$ & Simple & $\begin{array}{l}\text { Exponential } \\
\text { Moving Av. }\end{array}$ \\
\hline & Jarque-Bera & .00 & .00 & .00 & .15 & .01 & .00 & .11 & .08 & .00 & .00 \\
\hline \multirow[t]{3}{*}{ Log-volatility } & Kolmogorov-Smirnov & .18 & .26 & .43 & .69 & .23 & .00 & .85 & .56 & .00 & .38 \\
\hline & Lilliefors & .01 & .02 & .07 & .27 & .01 & .00 & .50 & .15 & .00 & .50 \\
\hline & Anderson-Darling & .00 & .00 & .00 & .28 & .01 & .00 & .53 & .14 & .00 & .00 \\
\hline Devolatilized & Kolmogorov-Smirnov & .45 & .90 & 1.00 & .28 & .65 & .00 & .73 & .10 & .00 & .93 \\
\hline \multirow[t]{3}{*}{ Log-volatility } & Lilliefors & .08 & .50 & .50 & .02 & .23 & .00 & .32 & .00 & .00 & .50 \\
\hline & Anderson-Darling & .02 & .17 & .98 & .01 & .06 & .00 & .11 & .00 & .00 & .63 \\
\hline & Jarque-Bera & .00 & .00 & .00 & .50 & .00 & .00 & .01 & .00 & .00 & .00 \\
\hline DeGARCH & Kolmogorov-Smirnov & .03 & .04 & .35 & .91 & .61 & .00 & .96 & .01 & .00 & .00 \\
\hline Log-volatility & Anderson-Darling & .00 & .00 & .01 & .64 & .00 & .00 & .32 & .00 & .00 & .00 \\
\hline
\end{tabular}

Source: Euronext, 30' resampled intraday CAC40 French stock index quotes from the period 01-01-1997/05-31-2009. Are reported in this table the P-values of the Normality tests for the Log-volatility, the Devolatilized Log-volatility and the DeGARCH Log-volatility series. The four Normality tests correspond to the Jarque-Bera, the Kolmogorov-Smirnov, the Lilliefors and the Anderson-Darling tests (Bontemps and Meddahi, 2005). The "Log-volatility" series are computed as the standardized logarithm function of the volatility series. The "Devolatilized Log-volatility" represents the standardized logarithm function of volatility series, normalized using their standard deviation computed on a one-year rolling window. The "DeGARCH Log-volatility" corresponds to residual Log-volatility estimates computed from an AR(1)-GARCH(1,1) model. Computations by the authors. 
Table C.2-a: Estimates of Shape-parameters of Generalized Extreme Value Distributions

of Period Maxima of Daily Log-volatilities using a Method of Classical Moments

\begin{tabular}{|c|c|c|c|c|c|c|c|c|c|c|c|c|c|}
\hline Method & requencies & Series & Statistics & $\begin{array}{l}\text { Realized } \\
\text { Volatility }\end{array}$ & $\begin{array}{l}\text { Bi-power } \\
\text { Variation }\end{array}$ & $\begin{array}{l}\text { Jump } \\
\text { Comp. }\end{array}$ & Parkinson & Kunitomo & $\begin{array}{l}\text { Rogers- } \\
\text { Satchell } \\
\end{array}$ & $\begin{array}{c}\text { Garman- } \\
\text { Klass } \\
\end{array}$ & $\begin{array}{l}\text { Yang- } \\
\text { Zhang } \\
\end{array}$ & Simple & $\begin{array}{l}\text { Exponential } \\
\text { Moving Av. }\end{array}$ \\
\hline \multirow{9}{*}{$\begin{array}{l}\text { Classical } \\
\text { moments }\end{array}$} & \multirow{3}{*}{ Weekly } & Original & $\begin{array}{c}\text { Shape-par. } \\
{[5 \% ; 95 \%]} \\
\text { KS P-stat. }\end{array}$ & $\begin{array}{c}-.18 \\
{[-.21 ;-.16]} \\
(.64)\end{array}$ & $\begin{array}{c}-.19 \\
{[-.22 ;-.15]} \\
(.15)\end{array}$ & $\begin{array}{c}-.16 \\
{[-.19 ;-.13]} \\
(.55) \\
\end{array}$ & $\begin{array}{c}-.22 \\
{[-.25 ;-.19]} \\
(.96)\end{array}$ & $\begin{array}{c}-.18 \\
{[-.21 ;-.15]} \\
(.60) \\
\end{array}$ & $\begin{array}{c}-.21 \\
{[-.24 ;-.19]} \\
(.10) \\
\end{array}$ & $\begin{array}{c}-.21 \\
{[-.23 ;-.18]} \\
(.05) \\
\end{array}$ & $\begin{array}{c}-.17 \\
{[-.19 ;-.14]} \\
(.87) \\
\end{array}$ & $\begin{array}{c}-.26 \\
{[-.31 ;-.21]} \\
(.60) \\
\end{array}$ & $\begin{array}{c}-.19 \\
{[-.22 ;-.16]} \\
(.22) \\
\end{array}$ \\
\hline & & Bootstrap & $\begin{array}{c}\text { Shape-par. } \\
{[5 \% ; 95 \%]} \\
\text { KS P-stat. }\end{array}$ & $\begin{array}{c}-.08 \\
{[-.11 ;-.04]} \\
(.60) \\
\end{array}$ & $\begin{array}{c}-.14 \\
{[-.19 ;-.08]} \\
(.59) \\
\end{array}$ & $\begin{array}{c}-.14 \\
{[-.18 ;-.10]} \\
(.62) \\
\end{array}$ & $\begin{array}{c}-.17 \\
{[-.21 ;-.12]} \\
(.59) \\
\end{array}$ & $\begin{array}{c}-.16 \\
{[-.21 ;-.12]} \\
(.58) \\
\end{array}$ & $\begin{array}{c}-.23 \\
{[-.29 ;-.18]} \\
(.56) \\
\end{array}$ & $\begin{array}{c}-.18 \\
{[-.23 ;-.13]} \\
(.57) \\
\end{array}$ & $\begin{array}{c}-.16 \\
{[-.22 ;-.10]} \\
(.56) \\
\end{array}$ & $\begin{array}{c}-.33 \\
{[-.40 ;-.27]} \\
(.48) \\
\end{array}$ & $\begin{array}{c}-.26 \\
{[-.31 ;-.20]} \\
(.54) \\
\end{array}$ \\
\hline & & Surrogate & $\begin{array}{c}\text { Shape-par. } \\
{[5 \% ; 95 \%]} \\
\text { KS P-stat. }\end{array}$ & $\begin{array}{c}-.07 \\
{[-.10 ;-.05]} \\
(.63)\end{array}$ & $\begin{array}{c}-.13 \\
{[-.19 ;-.08]} \\
(.55)\end{array}$ & $\begin{array}{c}-.14 \\
{[-.16 ;-.11]} \\
(.63) \\
\end{array}$ & $\begin{array}{c}-.17 \\
{[-.20 ;-.13]} \\
(.61) \\
\end{array}$ & $\begin{array}{c}-.16 \\
{[-.20 ;-.13]} \\
(.58) \\
\end{array}$ & $\begin{array}{c}-.24 \\
{[-.30 ;-.18]} \\
(.54) \\
\end{array}$ & $\begin{array}{c}-.18 \\
{[-.23 ;-.14]} \\
(.58) \\
\end{array}$ & $\begin{array}{c}-.16 \\
{[-.21 ;-.10]} \\
(.57) \\
\end{array}$ & $\begin{array}{c}-.33 \\
{[-.40 ;-.27]} \\
(.47) \\
\end{array}$ & $\begin{array}{c}-.26 \\
{[-.31 ;-.21]} \\
(.52) \\
\end{array}$ \\
\hline & \multirow{3}{*}{ Monthly } & Original & $\begin{array}{c}\text { Shape-par. } \\
{[5 \% ; 95 \%]} \\
\text { KS P-stat. }\end{array}$ & $\begin{array}{c}-.20 \\
{[-.24 ;-.15]} \\
(.49) \\
\end{array}$ & $\begin{array}{c}-.18 \\
{[-.24 ;-.12]} \\
(.89) \\
\end{array}$ & $\begin{array}{c}-.16 \\
{[-.21 ;-.10]} \\
(.49) \\
\end{array}$ & $\begin{array}{c}-.23 \\
{[-.28 ;-.16]} \\
(.70) \\
\end{array}$ & $\begin{array}{c}-.19 \\
{[-.24 ;-.13]} \\
(.70) \\
\end{array}$ & $\begin{array}{c}-.21 \\
{[-.27 ;-.15]} \\
(.80) \\
\end{array}$ & $\begin{array}{c}-.23 \\
{[-.29 ;-.18]} \\
(.80) \\
\end{array}$ & $\begin{array}{c}-.21 \\
{[-.26 ;-.15]} \\
(.18) \\
\end{array}$ & $\begin{array}{c}-.16 \\
{[-.22 ;-.11]} \\
(.80) \\
\end{array}$ & $\begin{array}{c}-.16 \\
{[-.21 ;-.09]} \\
(.95) \\
\end{array}$ \\
\hline & & Bootstrap & $\begin{array}{c}\text { Shape-par. } \\
{[5 \% ; 95 \%]} \\
\text { KS P-stat. }\end{array}$ & $\begin{array}{c}-.09 \\
{[-.16 ;-.04]} \\
(.60) \\
\end{array}$ & $\begin{array}{c}-.10 \\
{[-.21 ; .00]} \\
(.64) \\
\end{array}$ & $\begin{array}{c}-.14 \\
{[-.21 ;-.08]} \\
(.63) \\
\end{array}$ & $\begin{array}{c}-.14 \\
{[-.23 ;-.07]} \\
(.64) \\
\end{array}$ & $\begin{array}{c}-.14 \\
{[-.22 ;-.07]} \\
(.63) \\
\end{array}$ & $\begin{array}{c}-.15 \\
{[-.25 ;-.05]} \\
(.61) \\
\end{array}$ & $\begin{array}{c}-.14 \\
{[-.23 ;-.06]} \\
(.64) \\
\end{array}$ & $\begin{array}{c}-.10 \\
{[-.21 ; .00]} \\
(.62) \\
\end{array}$ & $\begin{array}{c}-.18 \\
{[-.28 ;-.09]} \\
(.63) \\
\end{array}$ & $\begin{array}{c}-.18 \\
{[-.28 ;-.08]} \\
(.64) \\
\end{array}$ \\
\hline & & Surrogate & $\begin{array}{c}\text { Shape-par. } \\
{[5 \% ; 95 \%]} \\
\text { KS P-stat. }\end{array}$ & $\begin{array}{c}-.09 \\
{[-.13 ;-.05]} \\
(.60) \\
\end{array}$ & $\begin{array}{c}-.09 \\
{[-.20 ; .00]} \\
(.62) \\
\end{array}$ & $\begin{array}{c}-.14 \\
{[-.19 ;-.09]} \\
(.63) \\
\end{array}$ & $\begin{array}{c}-.14 \\
{[-.21 ;-.07]} \\
(.63) \\
\end{array}$ & $\begin{array}{c}-.13 \\
{[-.20 ;-.07]} \\
(.64) \\
\end{array}$ & $\begin{array}{c}-.15 \\
{[-.25 ;-.05]} \\
(.63) \\
\end{array}$ & $\begin{array}{c}-.14 \\
{[-.23 ;-.06]} \\
(.64) \\
\end{array}$ & $\begin{array}{c}-.09 \\
{[-.20 ; .00]} \\
(.62) \\
\end{array}$ & $\begin{array}{c}-.18 \\
{[-.27 ;-.09]} \\
(.64) \\
\end{array}$ & $\begin{array}{c}-.17 \\
{[-.27 ;-.09]} \\
(.64) \\
\end{array}$ \\
\hline & \multirow{3}{*}{ Quarterly } & Original & $\begin{array}{c}\text { Shape-par. } \\
{[5 \% ; 95 \%]} \\
\text { KS P-stat. }\end{array}$ & $\begin{array}{c}-.28 \\
{[-.36 ;-.19]} \\
(.95) \\
\end{array}$ & $\begin{array}{c}-.21 \\
{[-.27 ;-.12]} \\
(.99) \\
\end{array}$ & $\begin{array}{c}-.21 \\
{[-.31 ;-.13]} \\
(.82) \\
\end{array}$ & $\begin{array}{c}-.31 \\
{[-.40 ;-.19]} \\
(1.00) \\
\end{array}$ & $\begin{array}{c}-.28 \\
{[-.38 ;-.20]} \\
(.95) \\
\end{array}$ & $\begin{array}{c}-. .30 \\
{[-.40 ;--21]} \\
(.82) \\
\end{array}$ & $\begin{array}{c}-.32 \\
{[-.40 ;-.23]} \\
(.65) \\
\end{array}$ & $\begin{array}{c}-. .33 \\
{[-.43 ;-.22]} \\
(.82) \\
\end{array}$ & $\begin{array}{c}-.17 \\
{[-.26 ;--09]} \\
(.48) \\
\end{array}$ & $\begin{array}{c}-.17 \\
{[-.26 ;-.09]} \\
(.99) \\
\end{array}$ \\
\hline & & Bootstrap & $\begin{array}{c}\text { Shape-par. } \\
{[5 \% ; 95 \%]} \\
\text { KS P-stat. }\end{array}$ & $\begin{array}{c}-.16 \\
{[-.25 ;-.07]} \\
(.62) \\
\end{array}$ & $\begin{array}{c}-.11 \\
{[-.25 ; .00]} \\
(.64) \\
\end{array}$ & $\begin{array}{c}-.19 \\
{[-.30 ;-.09]} \\
(.64) \\
\end{array}$ & $\begin{array}{c}-.18 \\
{[-.31 ;-.06]} \\
(.65) \\
\end{array}$ & $\begin{array}{c}-.18 \\
{[-.31 ;-.06]} \\
(.64) \\
\end{array}$ & $\begin{array}{c}-.16 \\
{[-.31 ; .00]} \\
(.66) \\
\end{array}$ & $\begin{array}{c}-.17 \\
{[-.30 ;-.04]} \\
(.65) \\
\end{array}$ & $\begin{array}{c}-.10 \\
{[-.26 ; .00]} \\
(.63) \\
\end{array}$ & $\begin{array}{c}-.19 \\
{[-.33 ;-.06]} \\
(.65) \\
\end{array}$ & $\begin{array}{c}-.18 \\
{[-.32 ;-.05]} \\
(.64) \\
\end{array}$ \\
\hline & & Surrogate & $\begin{array}{c}\text { Shape-par. } \\
{[5 \% ; 95 \%]} \\
\text { KS P-stat. }\end{array}$ & $\begin{array}{c}-.16 \\
{[-.23 ;-.09]} \\
(.68) \\
\end{array}$ & $\begin{array}{c}-.10 \\
{[-.24 ; .00]} \\
(.64) \\
\end{array}$ & $\begin{array}{c}-.19 \\
{[-.27 ;-.10]} \\
(.66) \\
\end{array}$ & $\begin{array}{c}-.17 \\
{[-.29 ;-.06]} \\
(.64) \\
\end{array}$ & $\begin{array}{c}-.17 \\
{[-.29 ;-.07]} \\
(.64) \\
\end{array}$ & $\begin{array}{c}-.15 \\
{[-.29 ; .00]} \\
(.65) \\
\end{array}$ & $\begin{array}{c}-.16 \\
{[-.29 ;-.04]} \\
(.66) \\
\end{array}$ & $\begin{array}{c}-.09 \\
{[-.25 ; .00]} \\
(.64) \\
\end{array}$ & $\begin{array}{c}-.18 \\
{[-.31 ;-.06]} \\
(.65) \\
\end{array}$ & $\begin{array}{c}-.17 \\
{[-.30 ;-.05]} \\
(.66) \\
\end{array}$ \\
\hline
\end{tabular}

Source: Euronext, 30' resampled intraday CAC40 French stock index quotes from the period 01-01-1997/05-31-2009. Reported in this table are the empirical Shape-parameters for the various volatility measures estimated by a Method of C-moments of a GEV density with various frequencies (the block maxima lengths). The $90 \%$ confidence intervals of Tail-indexes are reported in brackets, whilst P-statistics of Goodness-of-Fit Kolmogorov-Smirnov tests (denoted KS P-stat.) are between parentheses. The curly brackets (below the Tail-index estimations of the original series) are obtained using the "Accelerated Bootstrap" procedure by Gilli and Këllezi (2006) applied on volatility series. Computations by the authors. 
Table C.2-b: Estimates of Shape-parameters of Generalized Extreme Value Distributions of Period Maxima of Daily Log-volatilities using a Method of L-moments

\begin{tabular}{|c|c|c|c|c|c|c|c|c|c|c|c|c|c|}
\hline Method & Frequencies & Series & Statistics & $\begin{array}{l}\text { Realized } \\
\text { Volatility }\end{array}$ & $\begin{array}{l}\text { Bi-power } \\
\text { Variation }\end{array}$ & $\begin{array}{l}\text { Jump } \\
\text { Comp. }\end{array}$ & Parkinson & Kunitomo & $\begin{array}{l}\text { Rogers- } \\
\text { Satchell }\end{array}$ & $\begin{array}{c}\text { Garman- } \\
\text { Klass }\end{array}$ & $\begin{array}{l}\text { Yang- } \\
\text { Zhang }\end{array}$ & Simple & $\begin{array}{l}\text { Exponential } \\
\text { Moving Av. }\end{array}$ \\
\hline \multirow{9}{*}{ L-moments } & \multirow{3}{*}{ Weekly } & Original & $\begin{array}{c}\text { Shape-par. } \\
{[5 \% ; 95 \%]} \\
\text { KS P-stat. }\end{array}$ & $\begin{array}{c}-.19 \\
{[-.22 ;-.15]} \\
(.87)\end{array}$ & $\begin{array}{c}-.21 \\
{[-.24 ;-.17]} \\
(.60)\end{array}$ & $\begin{array}{c}-.16 \\
{[-.20 ;-.13]} \\
(.93) \\
\end{array}$ & $\begin{array}{c}-.22 \\
{[-.26 ;-.19]} \\
(.64)\end{array}$ & $\begin{array}{c}-.17 \\
{[-.21 ;-.14]} \\
(.25)\end{array}$ & $\begin{array}{c}-.21 \\
{[-.24 ;-.17]} \\
(.93) \\
\end{array}$ & $\begin{array}{c}-.21 \\
{[-.24 ;-. .17]} \\
(.46) \\
\end{array}$ & $\begin{array}{c}-.17 \\
{[-.20 ;-.13]} \\
(.69) \\
\end{array}$ & $\begin{array}{c}-.24 \\
{[-.28 ;-.20]} \\
(.97) \\
\end{array}$ & $\begin{array}{c}-.20 \\
{[-.24 ;-.17]} \\
(.60) \\
\end{array}$ \\
\hline & & Bootstrap & $\begin{array}{c}\text { Shape-par. } \\
{[5 \% ; 95 \%]} \\
\text { KS P-stat. }\end{array}$ & $\begin{array}{c}-.08 \\
{[-.12 ;-.03]} \\
(.62) \\
\end{array}$ & $\begin{array}{c}-.15 \\
{[-.20 ;-.10]} \\
(.60) \\
\end{array}$ & $\begin{array}{c}-.14 \\
{[-.19 ;-.09]} \\
(.64) \\
\end{array}$ & $\begin{array}{c}-.17 \\
{[-.22 ;-.13]} \\
(.63) \\
\end{array}$ & $\begin{array}{c}-.17 \\
{[-.22 ;-.12]} \\
(.60) \\
\end{array}$ & $\begin{array}{c}-.24 \\
{[-.29 ;-.19]} \\
(.59) \\
\end{array}$ & $\begin{array}{c}-.19 \\
{[-.24 ;-.14]} \\
(.61) \\
\end{array}$ & $\begin{array}{c}-.18 \\
{[-.23 ;-.13]} \\
(.62) \\
\end{array}$ & $\begin{array}{c}-.32 \\
{[-.38 ;-.27]} \\
(.55) \\
\end{array}$ & $\begin{array}{c}-.26 \\
{[-.32 ;-.21]} \\
(.57) \\
\end{array}$ \\
\hline & & Surrogate & $\begin{array}{c}\text { Shape-par. } \\
{[5 \% ; 95 \%]} \\
\text { KS P-stat. }\end{array}$ & $\begin{array}{c}-.08 \\
{[-.11 ;-.04]} \\
(.65)\end{array}$ & $\begin{array}{c}-.15 \\
{[-.20 ;-.10]} \\
(.60)\end{array}$ & $\begin{array}{c}-.14 \\
{[-.17 ;-.10]} \\
(.64) \\
\end{array}$ & $\begin{array}{c}-.18 \\
{[-.22 ;-.13]} \\
(.62)\end{array}$ & $\begin{array}{c}-.17 \\
{[-.21 ;-.13]} \\
(.59) \\
\end{array}$ & $\begin{array}{c}-.24 \\
{[-.30 ;-.19]} \\
(.58) \\
\end{array}$ & $\begin{array}{c}-.19 \\
{[-.24 ;-.15]} \\
(.60) \\
\end{array}$ & $\begin{array}{c}-.18 \\
{[-.23 ;-.13]} \\
(.61) \\
\end{array}$ & $\begin{array}{c}-.32 \\
{[-.38 ;-.27]} \\
(.52) \\
\end{array}$ & $\begin{array}{c}-.26 \\
{[-.31 ;-.21]} \\
(.57) \\
\end{array}$ \\
\hline & \multirow{3}{*}{ Monthly } & Original & $\begin{array}{c}\text { Shape-par. } \\
{[5 \% ; 95 \%]} \\
\text { KS P-stat. }\end{array}$ & $\begin{array}{c}-.19 \\
{[-.26 ;-.11]} \\
(.80) \\
\end{array}$ & $\begin{array}{c}-.19 \\
{[-.27 ;-.11]} \\
(.39) \\
\end{array}$ & $\begin{array}{c}-.14 \\
{[-.22 ;-.07]} \\
(.04) \\
\end{array}$ & $\begin{array}{c}-.26 \\
{[-.33 ;-.17]} \\
(.70) \\
\end{array}$ & $\begin{array}{c}-.16 \\
{[-.24 ;-.08]} \\
(.80) \\
\end{array}$ & $\begin{array}{c}-.20 \\
{[-.28 ;-.12]} \\
(.39) \\
\end{array}$ & $\begin{array}{c}-.22 \\
{[-.30 ;-. .15]} \\
(.80) \\
\end{array}$ & $\begin{array}{c}-.20 \\
{[-.27 ;-.13]} \\
(.89) \\
\end{array}$ & $\begin{array}{c}-.17 \\
{[-.26 ;-.10]} \\
(.99) \\
\end{array}$ & $\begin{array}{c}-.17 \\
{[-.25 ;-.10]} \\
(.95) \\
\end{array}$ \\
\hline & & Bootstrap & $\begin{array}{c}\text { Shape-par. } \\
{[5 \% ; 95 \%]} \\
\text { KS P-stat. }\end{array}$ & $\begin{array}{c}-.05 \\
{[-.14 ; .04]} \\
(.63) \\
\end{array}$ & $\begin{array}{c}-.09 \\
{[-.21 ; .02]} \\
(.67) \\
\end{array}$ & $\begin{array}{c}-.11 \\
{[-.21 ;-.02]} \\
(.64) \\
\end{array}$ & $\begin{array}{c}-.12 \\
{[-.23 ;-.03]} \\
(.65) \\
\end{array}$ & $\begin{array}{c}-.12 \\
{[-.23 ;-.03]} \\
(.65) \\
\end{array}$ & $\begin{array}{c}-.15 \\
{[-.26 ;-.05]} \\
(.65) \\
\end{array}$ & $\begin{array}{c}-.13 \\
{[-.24 ;-.03]} \\
(.64) \\
\end{array}$ & $\begin{array}{c}-.10 \\
{[-.23 ; .01]} \\
(.64) \\
\end{array}$ & $\begin{array}{c}-.17 \\
{[-.29 ;-.07]} \\
(.64) \\
\end{array}$ & $\begin{array}{c}-.17 \\
{[-.28 ;-.06]} \\
(.65) \\
\end{array}$ \\
\hline & & Surrogate & $\begin{array}{c}\text { Shape-par. } \\
{[5 \% ; 95 \%]} \\
\text { KS P-stat. }\end{array}$ & $\begin{array}{c}-.04 \\
{[-.11 ; .03]} \\
(.64) \\
\end{array}$ & $\begin{array}{c}-.09 \\
{[-.20 ; .03]} \\
(.65) \\
\end{array}$ & $\begin{array}{c}-.11 \\
{[-.19 ;-.04]} \\
(.67) \\
\end{array}$ & $\begin{array}{c}-.11 \\
{[-.20 ;-.02]} \\
(.66) \\
\end{array}$ & $\begin{array}{c}-.11 \\
{[-.19 ;-.02]} \\
(.65) \\
\end{array}$ & $\begin{array}{c}-.15 \\
{[-.25 ;-.04]} \\
(.65) \\
\end{array}$ & $\begin{array}{c}-.12 \\
{[-.23 ;-.03]} \\
(.65) \\
\end{array}$ & $\begin{array}{c}-.09 \\
{[-.20 ; .02]} \\
(.63) \\
\end{array}$ & $\begin{array}{c}-. .17 \\
{[-.27 ;-.07]} \\
(.65) \\
\end{array}$ & $\begin{array}{c}-.16 \\
{[-.27 ;--06]} \\
(.64) \\
\end{array}$ \\
\hline & \multirow{3}{*}{ Quarterly } & Original & $\begin{array}{c}\text { Shape-par. } \\
{[5 \% ; 95 \%]} \\
\text { KS P-stat. }\end{array}$ & $\begin{array}{c}-.28 \\
{[-.38 ;-.15]} \\
(.65) \\
\end{array}$ & $\begin{array}{c}-.19 \\
{[-.29 ;-.08]} \\
(.33) \\
\end{array}$ & $\begin{array}{c}-.21 \\
{[-.35 ;-.08]} \\
(.82) \\
\end{array}$ & $\begin{array}{c}-.31 \\
{[-.42 ;-.16]} \\
(.65) \\
\end{array}$ & $\begin{array}{c}-.29 \\
{[-.42 ;-.16]} \\
(.82) \\
\end{array}$ & $\begin{array}{c}-.32 \\
{[-.46 ;-.19]} \\
(.65) \\
\end{array}$ & $\begin{array}{c}-.34 \\
{[-.46 ;-.20]} \\
(.33) \\
\end{array}$ & $\begin{array}{c}-. .37 \\
{[-.52 ;-.21]} \\
(.48) \\
\end{array}$ & $\begin{array}{c}-. .15 \\
{[-.27 ;-.03]} \\
(.08) \\
\end{array}$ & $\begin{array}{c}-. .14 \\
{[-.26 ;-.01]} \\
(.14) \\
\end{array}$ \\
\hline & & Bootstrap & $\begin{array}{c}\text { Shape-par. } \\
{[5 \% ; 95 \%]} \\
\text { KS P-stat. }\end{array}$ & $\begin{array}{c}-.12 \\
{[-.27 ; .02]} \\
(.66) \\
\end{array}$ & $\begin{array}{c}-.06 \\
{[-.24 ; .11]} \\
(.65) \\
\end{array}$ & $\begin{array}{c}-.15 \\
{[-.31 ; .00]} \\
(.67) \\
\end{array}$ & $\begin{array}{c}-.15 \\
{[-.32 ; .02]} \\
(.65) \\
\end{array}$ & $\begin{array}{c}-.15 \\
{[-.32 ; .01]} \\
(.66) \\
\end{array}$ & $\begin{array}{c}-.13 \\
{[-.32 ; .05]} \\
(.65) \\
\end{array}$ & $\begin{array}{c}-.14 \\
{[-.30 ; .03]} \\
(.67) \\
\end{array}$ & $\begin{array}{c}-.07 \\
{[-.26 ; .11]} \\
(.66) \\
\end{array}$ & $\begin{array}{c}-.17 \\
{[-.35 ; 00]} \\
(.66) \\
\end{array}$ & $\begin{array}{c}-.16 \\
{[-.33 ; .01]} \\
(.65) \\
\end{array}$ \\
\hline & & Surrogate & $\begin{array}{c}\text { Shape-par. } \\
{[5 \% ; 95 \%]} \\
\text { KS P-stat. }\end{array}$ & $\begin{array}{c}-.10 \\
{[-.21 ; .00]} \\
(.68) \\
\end{array}$ & $\begin{array}{c}-.05 \\
{[-.22 ; .12]} \\
(.66) \\
\end{array}$ & $\begin{array}{c}-.14 \\
{[-.27 ;-.01]} \\
(.67) \\
\end{array}$ & $\begin{array}{c}-.13 \\
{[-.29 ; .01]} \\
(.65) \\
\end{array}$ & $\begin{array}{c}-.13 \\
{[-.28 ; .01]} \\
(.66) \\
\end{array}$ & $\begin{array}{c}-.12 \\
{[-.29 ; .05]} \\
\quad(.67) \\
\end{array}$ & $\begin{array}{c}-.12 \\
{[-.29 ; .04]} \\
(.66) \\
\end{array}$ & $\begin{array}{c}-.06 \\
{[-.24 ; .12]} \\
(.65) \\
\end{array}$ & $\begin{array}{c}-. .16 \\
{[-.33 ;-.01]} \\
(.66) \\
\end{array}$ & $\begin{array}{c}-.15 \\
{[-.31 ; .01]} \\
(.66) \\
\end{array}$ \\
\hline
\end{tabular}

Source: Euronext, 30' resampled intraday CAC40 French stock index quotes from the period 01-01-1997/05-31-2009. Reported in this table are the empirical Shape-parameters for the various volatility measures estimated by a Method of L-moments of a GEV density with various frequencies (the block maxima lengths). The 90\% confidence intervals of Tail-indexes are reported in brackets, whilst P-statistics of Goodness-of-Fit Kolmogorov-Smirnov tests (denoted KS P-stat.) are between parentheses. The curly brackets (below the Tail-index estimations of the original series) are obtained using the "Accelerated Bootstrap" procedure by Gilli and Këllezi (2006) applied on volatility series. Computations by the authors. 
Table C.2-c: Estimates of Shape-parameters of Generalized Extreme Value Distributions

of Period Maxima of Daily Log-volatilities using a Method of LQ-moments

\begin{tabular}{|c|c|c|c|c|c|c|c|c|c|c|c|c|c|}
\hline \multirow{28}{*}{ LQ-moments } & Frequencies & Series & Statistics & $\begin{array}{c}\text { Realized } \\
\text { Volatility }\end{array}$ & $\begin{array}{l}\text { Bi-power } \\
\text { Variation }\end{array}$ & $\begin{array}{l}\text { Jump } \\
\text { Comp. }\end{array}$ & Parkinson & Kunitomo & $\begin{array}{l}\text { Rogers- } \\
\text { Satchell }\end{array}$ & $\begin{array}{c}\text { Garman- } \\
\text { Klass }\end{array}$ & $\begin{array}{l}\text { Yang- } \\
\text { Zhang }\end{array}$ & Simple & $\begin{array}{l}\text { Exponential } \\
\text { Moving Av. }\end{array}$ \\
\hline & \multirow{9}{*}{ Weekly } & & Shape-par. & $\frac{-.16}{-.16}$ & -..31 & $\frac{-.21}{-.21}$ & - -.26 & "-.17 & - -.18 & $\frac{-.27}{-.27}$ & - & (-.24 & "-.27 \\
\hline & & Original & {$[5 \% ; 95 \%]$} & {$[-.24 ;-.08]$} & {$[-.38 ;-.23]$} & {$[-.31 ;-.14]$} & {$[-.34 ;-.18]$} & {$[-.26 ;-.09]$} & {$[-.25 ;-.07]$} & {$[-.37 ;-.18]$} & {$[-.26 ;-.08]$} & {$[-.34 ;-.17]$} & {$[-.35 ;-.19]$} \\
\hline & & & KS P-stat. & $(.55)$ & $(.87)$ & (.17) & (.96) & $(.83)$ & $(.87)$ & (.31) & $(.83)$ & $(.74)$ & $(.79)$ \\
\hline & & & Shape-par. & -.09 & -.17 & -.15 & -.19 & -.19 & -.25 & -.21 & -.20 & -.33 & -.28 \\
\hline & & Bootstrap & {$[5 \% ; 95 \%]$} & {$[-.21 ; .01]$} & {$[-.28 ;-.06]$} & {$[-.26 ;-.05]$} & {$[-.29 ;-.08]$} & {$[-.29 ;-.08]$} & {$[-.35 ;-.14]$} & {$[-.31 ;-.09]$} & {$[-.30 ;-.09]$} & {$[-.44 ;-.23]$} & {$[-.38 ;-.18]$} \\
\hline & & & KS P-stat. & $(.63)$ & $(.64)$ & $(.65)$ & (.65) & $(.64)$ & $(.65)$ & $(.64)$ & $(.65)$ & (.63) & (.64) \\
\hline & & & Shape-par. & -.09 & -.17 & -.15 & -.19 & -.19 & -.25 & -.21 & -.20 & -.34 & -.28 \\
\hline & & Surrogate & {$[5 \% ; 95 \%]$} & {$[-.19 ;-.01]$} & {$[-.27 ;-.06]$} & {$[-.25 ;-.05]$} & {$[-.29 ;-.09]$} & {$[-.29 ;-.09]$} & {$[-.36 ;-.15]$} & {$[-.32 ;-.11]$} & {$[-.30 ;-.09]$} & {$[-.44 ;-.22]$} & {$[-.39 ;-.18]$} \\
\hline & & & KS P-stat. & $(.65)$ & $(.63)$ & $(.66)$ & $(.65)$ & $(.65)$ & $(.65)$ & $(.64)$ & (.64) & $(.62)$ & (.64) \\
\hline & \multirow{9}{*}{ Monthly } & \multirow{3}{*}{ Original } & Shape-par. & -.14 & -.27 & -.16 & -.44 & -.06 & -.27 & -.20 & -.32 & -.41 & -.19 \\
\hline & & & {$[5 \% ; 95 \%]$} & {$[-.29 ; .08]$} & {$[-.47 ;-.11]$} & {$[-.35 ; .02]$} & {$[-.59 ;-.23]$} & {$[-.26 ; .17]$} & {$[-.47 ;-.13]$} & {$[-.33 ; .00]$} & {$[-.54 ;-.15]$} & {$[-.73 ;-.29]$} & {$[-.34 ; .02]$} \\
\hline & & & KS P-stat. & $(.24)$ & $(.80)$ & $(.31)$ & $(.39)$ & $(.59)$ & $(.31)$ & $(.70)$ & $(.89)$ & $(.80)$ & $(1.00)$ \\
\hline & & \multirow{3}{*}{ Bootstrap } & Shape-par. & .03 & -.09 & -.05 & -.10 & -.09 & -.15 & -.11 & -.12 & -.16 & -.15 \\
\hline & & & {$[5 \% ; 95 \%]$} & {$[-.22 ; .28]$} & {$[-.33 ; .16]$} & {$[-.27 ; .19]$} & {$[-.34 ; .13]$} & {$[-.33 ; .13]$} & {$[-.39 ; .07]$} & {$[-.35 ; .12]$} & {$[-.36 ; .11]$} & {$[-.38 ; .06]$} & {$[-.39 ; .06]$} \\
\hline & & & KS P-stat. & $(.64)$ & $(.66)$ & $(.64)$ & $(.65)$ & $(.64)$ & $(.66)$ & (.64) & (.65) & $(.66)$ & $(.67)$ \\
\hline & & \multirow{3}{*}{ Surrogate } & Shape-par. & .01 & -.10 & -.05 & -.09 & -.09 & -.15 & -.11 & -.11 & -.16 & -.16 \\
\hline & & & {$[5 \% ; 95 \%]$} & {$[-.17 ; .20]$} & {$[-.35 ; .14]$} & {$[-.24 ; .16]$} & {$[-.30 ; .12]$} & {$[-.30 ; .13]$} & {$[-.38 ; .08]$} & {$[-.32 ; .10]$} & {$[-.34 ; .12]$} & {$[-.39 ; .07]$} & {$[-.38 ; .07]$} \\
\hline & & & KS P-stat. & $(.65)$ & $(.66)$ & $(.67)$ & $(.65)$ & $(.65)$ & $(.67)$ & $(.65)$ & $(.66)$ & (.66) & $(.66)$ \\
\hline & \multirow{9}{*}{ Quarterly } & \multirow{3}{*}{ Original } & Shape-par. & -.30 & -.37 & -.30 & -.14 & -.39 & -.56 & -.33 & -.37 & -.02 & -.01 \\
\hline & & & {$[5 \% ; 95 \%]$} & {$[-.57 ;-.03]$} & {$[-.91 ;-.21]$} & {$[-.71 ;-.08]$} & {$[-.34 ; .31]$} & {$[-.77 ;-.18]$} & {$[-1.11 ;-.43]$} & {$[-.58 ;-.04]$} & {$[-.57 ; .02]$} & {$[-.30 ; .34]$} & {$[.31 ; .37]$} \\
\hline & & & KS P-stat. & $(.95)$ & $(.33)$ & $(.14)$ & $(.95)$ & $(.99)$ & (.48) & $(.95)$ & $(.22)$ & $(.82)$ & $(.99)$ \\
\hline & & \multirow{3}{*}{ Bootstrap } & Shape-par. & -.04 & -.08 & -.08 & -.08 & -.07 & -.14 & -.13 & -.07 & -.13 & -.12 \\
\hline & & & {$[5 \% ; 95 \%]$} & {$[-.40 ; .30]$} & {$[-.45 ; .27]$} & {$[-.44 ; .26]$} & {$[-.45 ; .26]$} & {$[-.45 ; .25]$} & {$[-.52 ; .20]$} & {$[-.47 ; .22]$} & {$[-.42 ; .27]$} & {$[-.48 ; .23]$} & {$[-.48 ; .24]$} \\
\hline & & & KS P-stat. & (.64) & (.68) & (.66) & $(.67)$ & (.66) & $(.67)$ & (.66) & (.66) & $(.67)$ & $(.68)$ \\
\hline & & \multirow{3}{*}{ Surrogate } & Shape-par. & -.06 & -.06 & -.09 & -.06 & -.05 & -.12 & -.10 & -.06 & -.12 & -.11 \\
\hline & & & {$[5 \% ; 95 \%]$} & {$[-.32 ; .20]$} & {$[-.42 ; .28]$} & {$[-.38 ; .21]$} & {$[-.37 ; .26]$} & {$[-.35 ; .27]$} & {$[-.49 ; .23]$} & {$[-.44 ; .24]$} & {$[-.42 ; .32]$} & {$[-.46 ; .23]$} & {$[-.44 ; .21]$} \\
\hline & & & KS P-stat. & $(.65)$ & $(.67)$ & $(.67)$ & $(.66)$ & $(.65)$ & $(.66)$ & $(.68)$ & $(.66)$ & $(.68)$ & $(.68)$ \\
\hline
\end{tabular}

Source: Euronext, 30' resampled intraday CAC40 French stock index quotes from the period 01-01-1997/05-31-2009. Reported in this table are the empirical Shape-parameters for the various volatility measures estimated by a Method of LQ-moments of a GEV density with various frequencies (the block maxima lengths). The $90 \%$ confidence intervals of Tail-indexes are reported in brackets, whilst P-statistics of Goodness-of-Fit Kolmogorov-Smirnov tests (denoted KS P-stat.) are between parentheses. The curly brackets (below the Tail-index estimations of the original series) are obtained using the "Accelerated Bootstrap" procedure by Gilli and Këllezi (2006) applied on volatility series. Computations by the authors. 
Table C.2-d: Estimates of Shape-parameters of Generalized Extreme Value Distributions

of Period Maxima of Daily Log-volatilities using a Method of LSd-moments

\begin{tabular}{|c|c|c|c|c|c|c|c|c|c|c|c|c|c|}
\hline Method & Frequencies & Series & Statistics & $\begin{array}{l}\text { Realized } \\
\text { Volatility } \\
\end{array}$ & $\begin{array}{l}\text { Bi-power } \\
\text { Variation } \\
\end{array}$ & $\begin{array}{l}\text { Jump } \\
\text { Comp. }\end{array}$ & Parkinson & Kunitomo & $\begin{array}{l}\text { Rogers- } \\
\text { Satchell }\end{array}$ & $\begin{array}{c}\text { Garman- } \\
\text { Klass }\end{array}$ & $\begin{array}{l}\text { Yang- } \\
\text { Zhang } \\
\end{array}$ & Simple & $\begin{array}{l}\text { Exponential } \\
\text { Moving Av. }\end{array}$ \\
\hline \multirow{9}{*}{ LSd-moments } & \multirow{3}{*}{ Weekly } & Original & $\begin{array}{c}\text { Shape-par. } \\
{[5 \% ; 95 \%]} \\
\text { KS P-stat. }\end{array}$ & $\begin{array}{c}-.20 \\
{[-.26 ;-.14]} \\
(.93) \\
\end{array}$ & $\begin{array}{c}-.21 \\
{[-.26 ;-.16]} \\
(.64) \\
\end{array}$ & $\begin{array}{c}-.16 \\
{[-.22 ;-.09]} \\
(.74) \\
\end{array}$ & $\begin{array}{c}-.23 \\
{[-.30 ;-.16]} \\
(.79) \\
\end{array}$ & $\begin{array}{c}-.19 \\
{[-.23 ;-.14]} \\
(.38) \\
\end{array}$ & $\begin{array}{c}-.21 \\
{[-.26 ;-.15]} \\
(.99) \\
\end{array}$ & $\begin{array}{c}-.21 \\
{[-.25 ;-.13]} \\
(.55) \\
\end{array}$ & $\begin{array}{c}-.17 \\
{[-.23 ;-.10]} \\
(.97) \\
\end{array}$ & $\begin{array}{c}-.22 \\
{[-.27 ;-.17]} \\
(1.00) \\
\end{array}$ & $\begin{array}{c}-.20 \\
{[-.25 ;-.14]} \\
(.17) \\
\end{array}$ \\
\hline & & Bootstrap & $\begin{array}{c}\text { Shape-par. } \\
{[5 \% ; 95 \%]} \\
\text { KS P-stat. }\end{array}$ & $\begin{array}{c}-.07 \\
{[-.12 ;-.02]} \\
(.62) \\
\end{array}$ & $\begin{array}{c}-.14 \\
{[-.19 ;-.09]} \\
(.65) \\
\end{array}$ & $\begin{array}{c}-.14 \\
{[-.19 ;-.08]} \\
(.66) \\
\end{array}$ & $\begin{array}{c}-.17 \\
{[-.22 ;-.11]} \\
(.64) \\
\end{array}$ & $\begin{array}{c}-.16 \\
{[-.21 ;-.10]} \\
(.63) \\
\end{array}$ & $\begin{array}{c}-.23 \\
{[-.28 ;-.17]} \\
(.63) \\
\end{array}$ & $\begin{array}{c}-.18 \\
{[-.23 ;-.13]} \\
(.61) \\
\end{array}$ & $\begin{array}{c}-.17 \\
{[-.22 ;-.11]} \\
(.63) \\
\end{array}$ & $\begin{array}{c}-.29 \\
{[-.35 ;-.24]} \\
(.60) \\
\end{array}$ & $\begin{array}{c}-.24 \\
{[-.30 ;-.19]} \\
(.61) \\
\end{array}$ \\
\hline & & Surrogate & $\begin{array}{c}\text { Shape-par. } \\
{[5 \% ; 95 \%]} \\
\text { KS P-stat. }\end{array}$ & $\begin{array}{c}-.07 \\
{[-.11 ;-.04]} \\
(.64) \\
\end{array}$ & $\begin{array}{c}-.14 \\
{[-.20 ;-.09]} \\
(.61) \\
\end{array}$ & $\begin{array}{c}-.13 \\
{[-.17 ;--10]} \\
(.65) \\
\end{array}$ & $\begin{array}{c}-.17 \\
{[-.21 ;-.12]} \\
(.62) \\
\end{array}$ & $\begin{array}{c}-.16 \\
{[-.21 ;-.12]} \\
(.62) \\
\end{array}$ & $\begin{array}{c}-.22 \\
{[-.28 ;-.18]} \\
(.63) \\
\end{array}$ & $\begin{array}{c}-.18 \\
{[-.23 ;-.14]} \\
(.63) \\
\end{array}$ & $\begin{array}{c}-.17 \\
{[-.22 ;-.12]} \\
(.64) \\
\end{array}$ & $\begin{array}{c}-.29 \\
{[-.34 ;--24]} \\
(.59) \\
\end{array}$ & $\begin{array}{c}-.25 \\
{[-.29 ;-.20]} \\
(.63) \\
\end{array}$ \\
\hline & \multirow{3}{*}{ Monthly } & Original & $\begin{array}{c}\text { Shape-par. } \\
{[5 \% ; 95 \%]} \\
\text { KS P-stat. }\end{array}$ & $\begin{array}{c}-.21 \\
{[-.26 ;-.14]} \\
(.89) \\
\end{array}$ & $\begin{array}{c}-.20 \\
{[-.24 ;--14]} \\
(.89) \\
\end{array}$ & $\begin{array}{c}-.14 \\
{[-.20 ;-.08]} \\
(.39) \\
\end{array}$ & $\begin{array}{c}-.28 \\
{[-.35 ;-.22]} \\
(.70) \\
\end{array}$ & $\begin{array}{c}-.21 \\
{[-.26 ;-.16]} \\
(.18) \\
\end{array}$ & $\begin{array}{c}-.24 \\
{[-.30 ;-.19]} \\
(.80) \\
\end{array}$ & $\begin{array}{c}-.26 \\
{[-.30 ;-.20]} \\
(.80) \\
\end{array}$ & $\begin{array}{c}-.23 \\
{[-.30 ;-.19]} \\
(.49) \\
\end{array}$ & $\begin{array}{c}-.18 \\
{[-.26 ;-.14]} \\
(.49) \\
\end{array}$ & $\begin{array}{c}-.17 \\
{[-.21 ;-.12]} \\
(.39) \\
\end{array}$ \\
\hline & & Bootstrap & $\begin{array}{c}\text { Shape-par. } \\
{[5 \% ; 95 \%]} \\
\text { KS P-stat. }\end{array}$ & $\begin{array}{c}-.04 \\
{[-.15 ; .06]} \\
(.63) \\
\end{array}$ & $\begin{array}{c}-.09 \\
{[-.22 ; .02]} \\
(.65) \\
\end{array}$ & $\begin{array}{c}-.11 \\
{[-.22 ; .00]} \\
(.67) \\
\end{array}$ & $\begin{array}{c}-.12 \\
{[-.22 ;-.02]} \\
(.64) \\
\end{array}$ & $\begin{array}{c}-.12 \\
{[-.22 ;-.02]} \\
(.65) \\
\end{array}$ & $\begin{array}{c}-.15 \\
{[-.26 ;--.04]} \\
(.65) \\
\end{array}$ & $\begin{array}{c}-.13 \\
{[-.23 ;-.02]} \\
(.67) \\
\end{array}$ & $\begin{array}{c}-.10 \\
{[-.22 ; .03]} \\
(.67) \\
\end{array}$ & $\begin{array}{c}-.17 \\
{[-.28 ;-.06]} \\
(.63) \\
\end{array}$ & $\begin{array}{c}-.16 \\
{[-.27 ;-.04]} \\
(.67) \\
\end{array}$ \\
\hline & & Surrogate & $\begin{array}{c}\text { Shape-par. } \\
{[5 \% ; 95 \%]} \\
\text { KS P-stat. }\end{array}$ & $\begin{array}{c}-.04 \\
{[-.11 ; .03]} \\
(.66) \\
\end{array}$ & $\begin{array}{c}-.09 \\
{[-.20 ; .02]} \\
(.67) \\
\end{array}$ & $\begin{array}{c}-.10 \\
{[-.19 ;-.02]} \\
\quad .67) \\
\end{array}$ & $\begin{array}{c}-.12 \\
{[-.22 ;-.03]} \\
(.66) \\
\end{array}$ & $\begin{array}{c}-.11 \\
{[-.21 ;-.03]} \\
(.66) \\
\end{array}$ & $\begin{array}{c}-.14 \\
{[-.26 ;-.02]} \\
(.65) \\
\end{array}$ & $\begin{array}{c}-.12 \\
{[-.23 ;-.02]} \\
(.68) \\
\end{array}$ & $\begin{array}{c}-.09 \\
{[-.20 ; .03]} \\
(.65) \\
\end{array}$ & $\begin{array}{c}-.17 \\
{[-.28 ;-.05]} \\
(.65) \\
\end{array}$ & $\begin{array}{c}-.16 \\
{[-.27 ;-.06]} \\
(.67) \\
\end{array}$ \\
\hline & \multirow{3}{*}{ Quarterly } & Original & $\begin{array}{c}\text { Shape-par. } \\
{[5 \% ; 95 \%]} \\
\text { KS P-stat. }\end{array}$ & $\begin{array}{c}-.31 \\
{[-.37 ;-.26]} \\
(.95) \\
\end{array}$ & $\begin{array}{c}-.21 \\
{[-.27 ;-.16]} \\
(.48) \\
\end{array}$ & $\begin{array}{c}-.25 \\
{[-.30 ;-.20]} \\
(.82) \\
\end{array}$ & $\begin{array}{c}-.29 \\
{[-.34 ;-.23]} \\
(.65) \\
\end{array}$ & $\begin{array}{c}-.34 \\
{[-.40 ;-.28]} \\
(.48) \\
\end{array}$ & $\begin{array}{c}-.38 \\
{[-.43 ;-.32]} \\
(.65) \\
\end{array}$ & $\begin{array}{c}-.38 \\
{[-.45 ;-.32]} \\
(.82) \\
\end{array}$ & $\begin{array}{c}-.42 \\
{[-.48 ;-.37]} \\
(.95) \\
\end{array}$ & $\begin{array}{c}-.13 \\
{[-.17 ;-.07]} \\
(.82) \\
\end{array}$ & $\begin{array}{c}-.13 \\
{[-.20 ;-.09]} \\
(.95) \\
\end{array}$ \\
\hline & & Bootstrap & $\begin{array}{l}\text { Shape-par. } \\
{[5 \% ; 95 \%]} \\
\text { KS P-stat. }\end{array}$ & $\begin{array}{c}-.12 \\
{[-.29 ; .02]} \\
(.68) \\
\end{array}$ & $\begin{array}{c}-.08 \\
{[-.26 ; .09]} \\
(.67) \\
\end{array}$ & $\begin{array}{c}-.17 \\
{[-.33 ; .01]} \\
(.66) \\
\end{array}$ & $\begin{array}{c}-.16 \\
{[-.35 ; .01]} \\
(.66) \\
\end{array}$ & $\begin{array}{c}-.16 \\
{[-.35 ; .02]} \\
(.66) \\
\end{array}$ & $\begin{array}{c}-.15 \\
{[-.33 ; .04]} \\
(.68) \\
\end{array}$ & $\begin{array}{c}-.16 \\
{[-.32 ; .02]} \\
(.66) \\
\end{array}$ & $\begin{array}{c}-.07 \\
{[-.29 ; .12]} \\
(.68) \\
\end{array}$ & $\begin{array}{c}-.17 \\
{[-.37 ; .02]} \\
(.66) \\
\end{array}$ & $\begin{array}{c}-. .17 \\
{[-.37 ; .01]} \\
(.68) \\
\end{array}$ \\
\hline & & Surrogate & $\begin{array}{c}\text { Shape-par. } \\
{[5 \% ; 95 \%]} \\
\text { KS P-stat. }\end{array}$ & $\begin{array}{c}-.11 \\
{[-.23 ; .00]} \\
(.69) \\
\end{array}$ & $\begin{array}{c}-.07 \\
{[-.26 ; .12]} \\
(.65) \\
\end{array}$ & $\begin{array}{c}-.16 \\
{[-.29 ;-.01]} \\
\quad(.67) \\
\end{array}$ & $\begin{array}{c}-.16 \\
{[-.33 ; .01]} \\
(.67) \\
\end{array}$ & $\begin{array}{c}-.15 \\
{[-.32 ; .01]} \\
(.67) \\
\end{array}$ & $\begin{array}{c}-.14 \\
{[-.31 ; .04]} \\
(.68) \\
\end{array}$ & $\begin{array}{c}-.14 \\
{[-.30 ; .03]} \\
(.67) \\
\end{array}$ & $\begin{array}{c}-.07 \\
{[-.27 ; .13]} \\
(.66) \\
\end{array}$ & $\begin{array}{c}-.17 \\
{[-.35 ; .00]} \\
(.68) \\
\end{array}$ & $\begin{array}{c}-.16 \\
{[-.34 ; .00]} \\
(.66) \\
\end{array}$ \\
\hline
\end{tabular}

Source: Euronext, 30' resampled intraday CAC40 French stock index quotes from the period 01-01-1997/05-31-2009. Reported in this table are the empirical Shape-parameters for the various volatility measures estimated by a Method of LSd-moments of a GEV density with various frequencies (the block maxima lengths). The $90 \%$ confidence intervals of Tail-indexes are reported in brackets, whilst P-statistics of Goodness-of-Fit Kolmogorov-Smirnov tests (denoted KS P-stat.) are between parentheses. The curly brackets (below the Tail-index estimations of the original series) are obtained using the "Accelerated Bootstrap" procedure by Gilli and Këllezi (2006) applied on volatility series. Computations by the authors. 
Table C.3-a: Estimates of Shape-parameters of Generalized Pareto Distributions of Peaks-over-Threshold Daily Log-volatilities using a Method of Classical Moments

\begin{tabular}{|c|c|c|c|c|c|c|c|c|c|c|c|c|c|}
\hline \multirow{10}{*}{$\begin{array}{l}\text { Classical } \\
\text { moments }\end{array}$} & Frequencies & Series & Statistics & $\begin{array}{l}\text { Realized } \\
\text { Volatility } \\
\end{array}$ & $\begin{array}{l}\text { Bi-power } \\
\text { Variation } \\
\end{array}$ & $\begin{array}{l}\text { Jump } \\
\text { Comp. }\end{array}$ & Parkinson & Kunitomo & $\begin{array}{l}\text { Rogers- } \\
\text { Satchell } \\
\end{array}$ & $\begin{array}{c}\text { Garman- } \\
\text { Klass }\end{array}$ & $\begin{array}{l}\text { Yang- } \\
\text { Zhang }\end{array}$ & Simple & $\begin{array}{l}\text { Exponential } \\
\text { Moving Av. }\end{array}$ \\
\hline & & Original & $\begin{array}{c}\text { Shape-par. } \\
{[5 \% ; 95 \%]} \\
\text { KS P-stat. }\end{array}$ & $\begin{array}{c}-.25 \\
{[-.39 ; .13]} \\
(.91) \\
\end{array}$ & $\begin{array}{c}-.17 \\
{[-.31 ;-.04]} \\
(.30) \\
\end{array}$ & $\begin{array}{c}-.14 \\
{[-.29 ;-.01]} \\
(.15) \\
\end{array}$ & $\begin{array}{c}-.18 \\
{[-.33 ;-.05]} \\
(.67) \\
\end{array}$ & $\begin{array}{c}-.19 \\
{[-.33 ;-.06]} \\
(.22) \\
\end{array}$ & $\begin{array}{c}-. .33 \\
{[-.46 ;-.18]} \\
(.07) \\
\end{array}$ & $\begin{array}{c}-.42 \\
{[-.57 ;-.30]} \\
(.30) \\
\end{array}$ & $\begin{array}{c}-.23 \\
{[-.37 ;-.11]} \\
(.53) \\
\end{array}$ & $\begin{array}{c}-.22 \\
{[-.36 ;-.09]} \\
(.53) \\
\end{array}$ & $\begin{array}{c}-.22 \\
{[-.37 ;-.09]} \\
(.41) \\
\end{array}$ \\
\hline & Weekly & Bootstrap & $\begin{array}{l}\text { Shape-par. } \\
{[5 \% ; 95 \%]} \\
\text { KS P-stat. }\end{array}$ & $\begin{array}{c}-.23 \\
{[-.35 ;-.11]} \\
(.50)\end{array}$ & $\begin{array}{c}-.11 \\
{[-.30 ; .07]} \\
(.55)\end{array}$ & $\begin{array}{c}-.25 \\
{[-.39 ;-.13]} \\
(.50)\end{array}$ & $\begin{array}{c}-.21 \\
{[-.37 ;-.07]} \\
(.52)\end{array}$ & $\begin{array}{c}-.22 \\
{[-.37 ;-.07]} \\
(.51)\end{array}$ & $\begin{array}{c}-.16 \\
{[-.34 ; .01]} \\
(.55)\end{array}$ & $\begin{array}{c}-.19 \\
{[-.35 ;-.04]} \\
(.53)\end{array}$ & $\begin{array}{c}-.09 \\
{[-.31 ; .07]} \\
(.54)\end{array}$ & $\begin{array}{c}-.22 \\
{[-.39 ;-. .06]} \\
(.54)\end{array}$ & $\begin{array}{c}-.18 \\
{[-.33 ;-.05]} \\
(.54)\end{array}$ \\
\hline & & Surrogate & $\begin{array}{c}\text { Shape-par. } \\
{[5 \% ; 95 \%]} \\
\text { KS P-stat. }\end{array}$ & $\begin{array}{c}-.21 \\
{[-.27 ;-.16]} \\
(.59) \\
\end{array}$ & $\begin{array}{c}-.11 \\
{[-.29 ; .07]} \\
(.52) \\
\end{array}$ & $\begin{array}{c}-.25 \\
{[-.34 ;-.16]} \\
(.52) \\
\end{array}$ & $\begin{array}{c}-.21 \\
{[-.35 ;-.07]} \\
(.51) \\
\end{array}$ & $\begin{array}{c}-.21 \\
{[-.35 ;-.07]} \\
(.52) \\
\end{array}$ & $\begin{array}{c}-.15 \\
{[-.33 ; .01]} \\
(.55) \\
\end{array}$ & $\begin{array}{c}-.18 \\
{[-.34 ;-.05]} \\
(.53) \\
\end{array}$ & $\begin{array}{c}-.09 \\
{[-.28 ; .08]} \\
(.53) \\
\end{array}$ & $\begin{array}{c}-.20 \\
{[-.37 ;-.06]} \\
(.56) \\
\end{array}$ & $\begin{array}{c}-.18 \\
{[-.31 ;-.05]} \\
(.55) \\
\end{array}$ \\
\hline & & Original & $\begin{array}{c}\text { Shape-par. } \\
{[5 \% ; 95 \%]} \\
\text { KS P-stat. }\end{array}$ & $\begin{array}{c}-.15 \\
{[-.29 ;-.02]} \\
(.03) \\
\end{array}$ & $\begin{array}{c}-.10 \\
{[-.24 ; .03]} \\
(.83) \\
\end{array}$ & $\begin{array}{c}-.21 \\
{[-.35 ;-.08]} \\
(.11) \\
\end{array}$ & $\begin{array}{c}-.28 \\
{[-.42 ;-.14]} \\
(.95) \\
\end{array}$ & $\begin{array}{c}-.24 \\
{[-.37 ;-.11]} \\
(.90) \\
\end{array}$ & $\begin{array}{c}-.27 \\
{[-.41 ;-.14]} \\
(.19) \\
\end{array}$ & $\begin{array}{c}-.40 \\
{[-.54 ;-.27]} \\
(.55) \\
\end{array}$ & $\begin{array}{c}-.18 \\
{[-.31 ;-.04]} \\
(.64) \\
\end{array}$ & $\begin{array}{c}-. .16 \\
{[-.30 ;-.02]} \\
(.55) \\
\end{array}$ & $\begin{array}{c}-.18 \\
{[-.32 ;-.04]} \\
(.90) \\
\end{array}$ \\
\hline & Monthly & Bootstrap & $\begin{array}{c}\text { Shape-par. } \\
{[5 \% ; 95 \%]} \\
\text { KS P-stat. }\end{array}$ & $\begin{array}{c}-.14 \\
{[-.23 ;-.06]} \\
(.34) \\
\end{array}$ & $\begin{array}{c}-.09 \\
{[-.24 ; .05]} \\
(.51) \\
\end{array}$ & $\begin{array}{c}-.17 \\
{[-.27 ;-.08]} \\
(.48) \\
\end{array}$ & $\begin{array}{c}-.16 \\
{[-.27 ;--.06]} \\
(.52) \\
\end{array}$ & $\begin{array}{c}-.16 \\
{[-.26 ;-.07]} \\
(.50) \\
\end{array}$ & $\begin{array}{c}-.14 \\
{[-.28 ;-.02]} \\
(.53) \\
\end{array}$ & $\begin{array}{c}-.15 \\
{[-.26 ;-.04]} \\
(.53) \\
\end{array}$ & $\begin{array}{c}-.08 \\
{[-.24 ; .05]} \\
(.51) \\
\end{array}$ & $\begin{array}{c}-.18 \\
{[-.31 ;-.07]} \\
(.54) \\
\end{array}$ & $\begin{array}{c}-.15 \\
{[-.25 ;-.05]} \\
(.54) \\
\end{array}$ \\
\hline & & Surrogate & $\begin{array}{c}\text { Shape-par. } \\
{[5 \% ; 95 \%]} \\
\text { KS P-stat. }\end{array}$ & $\begin{array}{c}-.13 \\
{[-.17 ;-.09]} \\
(.37) \\
\end{array}$ & $\begin{array}{c}-.09 \\
{[-.22 ; .05]} \\
(.50) \\
\end{array}$ & $\begin{array}{c}-.16 \\
{[-.22 ;-.11]} \\
(.52) \\
\end{array}$ & $\begin{array}{c}-.15 \\
{[-.25 ;-.06]} \\
(.52) \\
\end{array}$ & $\begin{array}{c}-.15 \\
{[-.24 ;-.07]} \\
(.52) \\
\end{array}$ & $\begin{array}{c}-.13 \\
{[-.27 ;-.01]} \\
(.54) \\
\end{array}$ & $\begin{array}{c}-.14 \\
{[-.25 ;-.05]} \\
(.54) \\
\end{array}$ & $\begin{array}{c}-.08 \\
{[-.22 ; .06]} \\
(.51) \\
\end{array}$ & $\begin{array}{c}-.18 \\
{[-.29 ;-.07]} \\
(.55) \\
\end{array}$ & $\begin{array}{c}-.14 \\
{[-.24 ;-.05]} \\
(.56) \\
\end{array}$ \\
\hline & & Original & $\begin{array}{c}\text { Shape-par. } \\
{[5 \% ; 95 \%]} \\
\text { KS P-stat. }\end{array}$ & $\begin{array}{c}-.14 \\
{[-.27 ;-.02]} \\
(.67) \\
\end{array}$ & $\begin{array}{c}-. .10 \\
{[-.23 ; .03]} \\
(.97) \\
\end{array}$ & $\begin{array}{c}-. .17 \\
{[-.32 ;-.03]} \\
(.26) \\
\end{array}$ & $\begin{array}{c}-.24 \\
{[-.36 ;-.11]} \\
(.07) \\
\end{array}$ & $\begin{array}{c}-.18 \\
{[-.31 ;-.04]} \\
(.26) \\
\end{array}$ & $\begin{array}{c}-.27 \\
{[-.39 ;-.15]} \\
(.67) \\
\end{array}$ & $\begin{array}{c}-.27 \\
{[-.40 ;-.17]} \\
(.09) \\
\end{array}$ & $\begin{array}{c}-.16 \\
{[-.31 ;-.02]} \\
(.74) \\
\end{array}$ & $\begin{array}{c}-.21 \\
{[-.33 ;-.09]} \\
(.67) \\
\end{array}$ & $\begin{array}{c}-.18 \\
{[-.32 ;-.03]} \\
(.91) \\
\end{array}$ \\
\hline & Quarterly & Bootstrap & $\begin{array}{l}\text { Shape-par. } \\
{[5 \% ; 95 \%]} \\
\text { KS P-stat. }\end{array}$ & $\begin{array}{c}-.09 \\
{[-.15 ;-.03]} \\
(.46) \\
\end{array}$ & $\begin{array}{c}-.09 \\
{[-.20 ; .02]} \\
(.50) \\
\end{array}$ & $\begin{array}{c}-.14 \\
{[-.21 ;-.08]} \\
(.50) \\
\end{array}$ & $\begin{array}{c}-.14 \\
{[-.22 ;-.07]} \\
(.53) \\
\end{array}$ & $\begin{array}{c}-.14 \\
{[-.22 ;-.07]} \\
(.53) \\
\end{array}$ & $\begin{array}{c}-.15 \\
{[-.25 ;-.06]} \\
(.50) \\
\end{array}$ & $\begin{array}{c}-.14 \\
{[-.22 ;-.06]} \\
(.53) \\
\end{array}$ & $\begin{array}{c}-.09 \\
{[-.21 ; .02]} \\
(.47) \\
\end{array}$ & $\begin{array}{c}-.18 \\
{[-.28 ;-.10]} \\
(.53) \\
\end{array}$ & $\begin{array}{c}-.14 \\
{[-.22 ;-.07]} \\
(.52) \\
\end{array}$ \\
\hline & & Surrogate & $\begin{array}{c}\text { Shape-par. } \\
{[5 \% ; 95 \%]} \\
\text { KS P-stat. }\end{array}$ & $\begin{array}{c}-.08 \\
{[-.11 ;-. .05]} \\
(.51) \\
\end{array}$ & $\begin{array}{c}-.09 \\
{[-.19 ; .02]} \\
(.49) \\
\end{array}$ & $\begin{array}{c}-.14 \\
{[-.18 ;-.10]} \\
(.54) \\
\end{array}$ & $\begin{array}{c}-.14 \\
{[-.20 ;-.07]} \\
(.53) \\
\end{array}$ & $\begin{array}{c}-.13 \\
{[-.20 ;-.07]} \\
(.54) \\
\end{array}$ & $\begin{array}{c}-.14 \\
{[-.24 ;-.04]} \\
(.50) \\
\end{array}$ & $\begin{array}{c}-.13 \\
{[-.21 ;-.05]} \\
(.53) \\
\end{array}$ & $\begin{array}{c}-.09 \\
{[-.20 ; .02]} \\
(.48) \\
\end{array}$ & $\begin{array}{c}-.18 \\
{[-.26 ;--10]} \\
(.54) \\
\end{array}$ & $\begin{array}{c}-.13 \\
{[-.22 ;-.06]} \\
(.56) \\
\end{array}$ \\
\hline
\end{tabular}

Source: Euronext, 30' resampled intraday CAC40 French stock index quotes from the period 01-01-1997/05-31-2009. Reported in this table are the empirical Shape-parameters for the various volatility measures. estimated by a Method of C-moments of a GPD with various quantiles of observations (thresholds for the peak definitions). The $90 \%$ confidence intervals are reported in brackets, whilst P-statistics of Goodness-of-Fit Kolmogorov-Smirnov tests (denoted KS P-stat.) are between parentheses. Computations by the authors. 
Table C.3-b: Estimates of Shape-parameters of Generalized Pareto Distributions

of Peaks-over-Threshold Daily Log-volatilities using a Method of L-moments

\begin{tabular}{|c|c|c|c|c|c|c|c|c|c|c|c|c|c|}
\hline L-moments & Frequencies & Series & Statistics & $\begin{array}{c}\text { Realized } \\
\text { Volatility } \\
\end{array}$ & $\begin{array}{c}\text { Bi-power } \\
\text { Variation } \\
\end{array}$ & $\begin{array}{l}\text { Jump } \\
\text { Comp. }\end{array}$ & Parkinson & Kunitomo & $\begin{array}{l}\text { Rogers- } \\
\text { Satchell }\end{array}$ & $\begin{array}{c}\text { Garman- } \\
\text { Klass }\end{array}$ & $\begin{array}{l}\text { Yang- } \\
\text { Zhang }\end{array}$ & Simple & $\begin{array}{l}\text { Exponential } \\
\text { Moving Av. }\end{array}$ \\
\hline \multirow{9}{*}{ L-moments } & \multirow{3}{*}{ Weekly } & Original & $\begin{array}{c}\text { Shape-par. } \\
{[5 \% ; 95 \%]} \\
\text { KS P-stat. }\end{array}$ & $\begin{array}{c}-.14 \\
{[-.29 ;-.02]} \\
(.91) \\
\end{array}$ & $\begin{array}{c}-.05 \\
{[-.17 ; .06]} \\
(.97) \\
\end{array}$ & $\begin{array}{c}-.17 \\
{[-.30 ;-.08]} \\
(.67) \\
\end{array}$ & $\begin{array}{c}-.26 \\
{[-.39 ;-.13]} \\
(.30) \\
\end{array}$ & $\begin{array}{c}-.15 \\
{[-.28 ;-.07]} \\
(.22) \\
\end{array}$ & $\begin{array}{c}-.49 \\
{[-.64 ;-.34]} \\
(.53) \\
\end{array}$ & $\begin{array}{c}-.52 \\
{[-.66 ;--.41]} \\
(.97) \\
\end{array}$ & $\begin{array}{c}-.21 \\
{[-.35 ; .10]} \\
(.41) \\
\end{array}$ & $\begin{array}{c}-.17 \\
{[-.29 ;-.06]} \\
(.22) \\
\end{array}$ & $\begin{array}{c}-.13 \\
{[-.26 ;-.06]} \\
(.41) \\
\end{array}$ \\
\hline & & Bootstrap & $\begin{array}{r}\text { Shape-par. } \\
{[5 \% ; 95 \%]} \\
\text { KS P-stat. }\end{array}$ & $\begin{array}{c}-.16 \\
{[-.34 ; .01]} \\
(.58) \\
\end{array}$ & $\begin{array}{c}-.08 \\
{[-.30 ; .11]} \\
(.60) \\
\end{array}$ & $\begin{array}{c}-.18 \\
{[-.39 ; .00]} \\
(.59) \\
\end{array}$ & $\begin{array}{c}-.15 \\
{[-.35 ; .03]} \\
(.59) \\
\end{array}$ & $\begin{array}{c}-.15 \\
{[-.34 ; .03]} \\
(.58) \\
\end{array}$ & $\begin{array}{c}-.14 \\
{[-.34 ; .06]} \\
(.60) \\
\end{array}$ & $\begin{array}{c}-.14 \\
{[-.34 ; .04]} \\
(.59) \\
\end{array}$ & $\begin{array}{c}-.07 \\
{[-.29 ; .13]} \\
(.60) \\
\end{array}$ & $\begin{array}{c}-.18 \\
{[-.38 ; .01]} \\
(.60) \\
\end{array}$ & $\begin{array}{c}-.15 \\
{[-.32 ; .02]} \\
(.60) \\
\end{array}$ \\
\hline & & Surrogate & $\begin{array}{c}\text { Shape-par. } \\
{[5 \% ; 95 \%]} \\
\text { KS P-stat. }\end{array}$ & $\begin{array}{c}-.14 \\
{[-.24 ;-.05]} \\
(.64) \\
\end{array}$ & $\begin{array}{c}-.07 \\
{[-.28 ; .11]} \\
(.59) \\
\end{array}$ & $\begin{array}{c}-.18 \\
{[-.31 ;-.05]} \\
(.60) \\
\end{array}$ & $\begin{array}{c}-.14 \\
{[-.31 ; .03]} \\
(.61) \\
\end{array}$ & $\begin{array}{c}-.13 \\
{[-.30 ; .03]} \\
(.60) \\
\end{array}$ & $\begin{array}{c}-.12 \\
{[-.32 ; .06]} \\
(.61) \\
\end{array}$ & $\begin{array}{c}-.13 \\
{[-.31 ; .04]} \\
(.61) \\
\end{array}$ & $\begin{array}{c}-.06 \\
{[-.27 ; .14]} \\
(.61) \\
\end{array}$ & $\begin{array}{c}-.16 \\
{[-.36 ; .01]} \\
(.60) \\
\end{array}$ & $\begin{array}{c}-.13 \\
{[-.29 ; .02]} \\
(.58) \\
\end{array}$ \\
\hline & & Original & $\begin{array}{c}\text { Shape-par. } \\
{[5 \% ; 95 \%]} \\
\text { KS P-stat. }\end{array}$ & $\begin{array}{c}-.04 \\
{[-.16 ;-.01]} \\
(.74) \\
\end{array}$ & $\begin{array}{c}-. .02 \\
{[-.12 ; .09]} \\
\quad(.64) \\
\end{array}$ & $\begin{array}{c}-.24 \\
{[-.18 ;--.02]} \\
(.64) \\
\end{array}$ & $\begin{array}{c}-. .32 \\
{[-.43 ;-.19]} \\
(.09) \\
\end{array}$ & $\begin{array}{c}-.23 \\
{[-.34 ;-.11]} \\
(.24) \\
\end{array}$ & $\begin{array}{c}-.25 \\
{[-.40 ;-.12]} \\
(.90) \\
\end{array}$ & $\begin{array}{c}-.38 \\
{[-.52 ;-.24]} \\
(.74) \\
\end{array}$ & $\begin{array}{c}-.11 \\
{[-.24 ; .01]} \\
(.30) \\
\end{array}$ & $\begin{array}{c}-.12 \\
{[-.25 ; .01]} \\
(.64) \\
\end{array}$ & $\begin{array}{c}-.15 \\
{[-.28 ;-.02]} \\
(.64) \\
\end{array}$ \\
\hline & Monthly & Bootstrap & $\begin{array}{c}\text { Shape-par. } \\
{[5 \% ; 95 \%]} \\
\text { KS P-stat. }\end{array}$ & $\begin{array}{c}-.04 \\
{[-.18 ; .09]} \\
(.54) \\
\end{array}$ & $\begin{array}{c}-.08 \\
{[-.23 ; .06]} \\
(.59) \\
\end{array}$ & $\begin{array}{c}-.10 \\
{[-.24 ; .03]} \\
(.60) \\
\end{array}$ & $\begin{array}{c}-.11 \\
{[-.25 ; .01]} \\
(.59) \\
\end{array}$ & $\begin{array}{c}-.11 \\
{[-.25 ; .01]} \\
(.58) \\
\end{array}$ & $\begin{array}{c}-.14 \\
{[-.28 ; .00]} \\
(.58) \\
\end{array}$ & $\begin{array}{c}-.12 \\
{[-.26 ; .01]} \\
(.59) \\
\end{array}$ & $\begin{array}{c}-.09 \\
{[-.25 ; .05]} \\
(.58) \\
\end{array}$ & $\begin{array}{c}-.16 \\
{[-.31 ;-.03]} \\
(.59) \\
\end{array}$ & $\begin{array}{c}-.12 \\
{[-.25 ;-.01]} \\
(.60) \\
\end{array}$ \\
\hline & & Surrogate & $\begin{array}{c}\text { Shape-par. } \\
{[5 \% ; 95 \%]} \\
\text { KS P-stat. }\end{array}$ & $\begin{array}{c}-.03 \\
{[-.10 ; .04]} \\
(.57) \\
\end{array}$ & $\begin{array}{c}-.08 \\
{[-.21 ; .06]} \\
(.59) \\
\end{array}$ & $\begin{array}{c}-.09 \\
{[-.18 ;-.01]} \\
(.60) \\
\end{array}$ & $\begin{array}{c}-.11 \\
{[-.22 ; .01]} \\
(.59) \\
\end{array}$ & $\begin{array}{c}-.10 \\
{[-.21 ; .01]} \\
(.60) \\
\end{array}$ & $\begin{array}{c}-.12 \\
{[-.28 ; .01]} \\
(.59) \\
\end{array}$ & $\begin{array}{c}-.11 \\
{[-.24 ; .01]} \\
(.58) \\
\end{array}$ & $\begin{array}{c}-.08 \\
{[-.24 ; .07]} \\
(.58) \\
\end{array}$ & $\begin{array}{c}-.16 \\
{[-.29 ;-.03]} \\
(.60) \\
\end{array}$ & $\begin{array}{c}-.12 \\
{[-.23 ; .00]} \\
(.61) \\
\end{array}$ \\
\hline & & Original & $\begin{array}{c}\text { Shape-par. } \\
{[5 \% ; 95 \%]} \\
\text { KS P-stat. }\end{array}$ & $\begin{array}{c}-.16 \\
{[-.28 ;-.08]} \\
(.11) \\
\end{array}$ & $\begin{array}{c}-.10 \\
{[-.22 ; .00]} \\
(1.00) \\
\end{array}$ & $\begin{array}{c}-.11 \\
{[-.18 ;-.02]} \\
(.16) \\
\end{array}$ & $\begin{array}{c}-.22 \\
{[-.30 ;-.15]} \\
(.95) \\
\end{array}$ & $\begin{array}{c}-.15 \\
{[-.24 ;-.01]} \\
(.22) \\
\end{array}$ & $\begin{array}{c}-.26 \\
{[-.37 ;--13]} \\
(.67) \\
\end{array}$ & $\begin{array}{c}-.20 \\
{[-.35 ;-.07]} \\
(.60) \\
\end{array}$ & $\begin{array}{c}-.14 \\
{[-.24 ;-.06]} \\
(.91) \\
\end{array}$ & $\begin{array}{c}-.25 \\
{[-.38 ;-. .11]} \\
(.54) \\
\end{array}$ & $\begin{array}{c}-.20 \\
{[-.30 ;-.13]} \\
(.41) \\
\end{array}$ \\
\hline & Quarterly & Bootstrap & $\begin{array}{c}\text { Shape-par. } \\
{[5 \% ; 95 \%]} \\
\text { KS P-stat. }\end{array}$ & $\begin{array}{c}-.04 \\
{[-.13 ; .05]} \\
(.55) \\
\end{array}$ & $\begin{array}{c}-.10 \\
{[-.21 ; .01]} \\
(.58) \\
\end{array}$ & $\begin{array}{c}-.12 \\
{[-.22 ;-.03]} \\
(.55) \\
\end{array}$ & $\begin{array}{c}-.13 \\
{[-.22 ;-.04]} \\
(.59) \\
\end{array}$ & $\begin{array}{c}-.12 \\
{[-.22 ;-.03]} \\
(.58) \\
\end{array}$ & $\begin{array}{c}-.16 \\
{[-.26 ;--.06]} \\
(.60) \\
\end{array}$ & $\begin{array}{c}-.13 \\
{[-.23 ;-.04]} \\
(.58) \\
\end{array}$ & $\begin{array}{c}-.11 \\
{[-.22 ;-.01]} \\
(.58) \\
\end{array}$ & $\begin{array}{c}-.19 \\
{[-.30 ;-.09]} \\
(.58) \\
\end{array}$ & $\begin{array}{c}-.13 \\
{[-.22 ;-.05]} \\
(.57) \\
\end{array}$ \\
\hline & & Surrogate & $\begin{array}{c}\text { Shape-par. } \\
{[5 \% ; 95 \%]} \\
\text { KS P-stat. }\end{array}$ & $\begin{array}{c}-.03 \\
{[-.08 ; .02]} \\
(.56) \\
\end{array}$ & $\begin{array}{c}-.09 \\
{[-.20 ; .01]} \\
(.56) \\
\end{array}$ & $\begin{array}{c}-.12 \\
{[-.19 ;-.05]} \\
(.57) \\
\end{array}$ & $\begin{array}{c}-.13 \\
{[-.21 ;-.04]} \\
(.57) \\
\end{array}$ & $\begin{array}{c}-.12 \\
{[-.21 ;-.04]} \\
(.58) \\
\end{array}$ & $\begin{array}{c}-.16 \\
{[-.27 ;--.06]} \\
(.56) \\
\end{array}$ & $\begin{array}{c}-.12 \\
{[-.22 ;-.03]} \\
(.58) \\
\end{array}$ & $\begin{array}{c}-.11 \\
{[-.22 ;-.01]} \\
(.58) \\
\end{array}$ & $\begin{array}{c}-.19 \\
{[-.28 ;-.10]} \\
(.58) \\
\end{array}$ & $\begin{array}{c}-.13 \\
{[-.22 ;-.04]} \\
(.58) \\
\end{array}$ \\
\hline
\end{tabular}

Source: Euronext, 30' resampled intraday CAC40 French stock index quotes from the period 01-01-1997/05-31-2009. Reported in this table are the empirical Shape-parameters for the various volatility measures. estimated by a Method of L-moments of a GPD with various quantiles of observations (thresholds for the peak definitions). The $90 \%$ confidence intervals are reported in brackets, whilst P-statistics of Goodness-of-Fit Kolmogorov-Smirnov tests (denoted KS P-stat.) are between parentheses. Computations by the authors. 
Table C.3-c: Estimates of Shape-parameters of Generalized Pareto Distributions

of Peaks-over-Threshold Daily Log-volatilities using a Method of LQ-moments

\begin{tabular}{|c|c|c|c|c|c|c|c|c|c|c|c|c|c|}
\hline Method & Frequencies & Series & Statistics & $\begin{array}{l}\text { Realized } \\
\text { Volatility }\end{array}$ & $\begin{array}{l}\text { Bi-power } \\
\text { Variation }\end{array}$ & $\begin{array}{l}\text { Jump } \\
\text { Comp. }\end{array}$ & Parkinson & Kunitomo & $\begin{array}{l}\text { Rogers- } \\
\text { Satchell }\end{array}$ & $\begin{array}{c}\text { Garman- } \\
\text { Klass }\end{array}$ & $\begin{array}{l}\text { Yang- } \\
\text { Zhang }\end{array}$ & Simple & $\begin{array}{l}\text { Exponential } \\
\text { Moving Av. }\end{array}$ \\
\hline \multirow{9}{*}{ LQ-moments } & \multirow{3}{*}{$2.50 \%$} & Original & $\begin{array}{c}\text { Shape-par. } \\
{[5 \% ; 95 \%]} \\
\text { KS P-stat. }\end{array}$ & $\begin{array}{c}-.22 \\
{[-.34 ;-.11]} \\
(.80) \\
\end{array}$ & $\begin{array}{c}-. .16 \\
{[-.29 ; .04]} \\
(.11) \\
\end{array}$ & $\begin{array}{c}-.34 \\
{[-.47 ;-.21]} \\
(.41) \\
\end{array}$ & $\begin{array}{c}-.47 \\
{[-.62 ;-.28]} \\
(.67) \\
\end{array}$ & $\begin{array}{c}-.05 \\
{[-.16 ; .08]} \\
(.11) \\
\end{array}$ & $\begin{array}{c}-.94 \\
{[-1.11 ;-.79]} \\
(.22)\end{array}$ & $\begin{array}{c}-.58 \\
{[-.74 ;-.42]} \\
(.11) \\
\end{array}$ & $\begin{array}{c}-.12 \\
{[-.24 ;-.07]} \\
(.22) \\
\end{array}$ & $\begin{array}{c}-.27 \\
{[-.41 ;-.20]} \\
(.53) \\
\end{array}$ & $\begin{array}{c}.01 \\
{[-.09 ; .14]} \\
(.03) \\
\end{array}$ \\
\hline & & Bootstrap & $\begin{array}{c}\text { Shape-par. } \\
\text { [5\%;95\%] } \\
\text { KS P-stat. }\end{array}$ & $\begin{array}{c}-.04 \\
{[-.50 ; .43]} \\
(.48) \\
\end{array}$ & $\begin{array}{c}-.07 \\
{[-.50 ; .35]} \\
(.55) \\
\end{array}$ & $\begin{array}{c}-.10 \\
{[-.55 ; .32]} \\
(.53) \\
\end{array}$ & $\begin{array}{c}-.04 \\
{[-.46 ; .37]} \\
(.54) \\
\end{array}$ & $\begin{array}{c}-.03 \\
{[-.44 ; .39]} \\
(.53) \\
\end{array}$ & $\begin{array}{c}-.11 \\
{[-.56 ; .29]} \\
(.51) \\
\end{array}$ & $\begin{array}{c}-.08 \\
{[-.51 ; .33]} \\
(.54) \\
\end{array}$ & $\begin{array}{c}-.06 \\
{[-.49 ; .34]} \\
(.53) \\
\end{array}$ & $\begin{array}{c}-.12 \\
{[-.55 ; .31]} \\
(.52) \\
\end{array}$ & $\begin{array}{c}-.09 \\
{[-.50 ; .28]} \\
(.54) \\
\end{array}$ \\
\hline & & Surrogate & $\begin{array}{c}\text { Shape-par. } \\
{[5 \% ; 95 \%]} \\
\text { KS P-stat. }\end{array}$ & $\begin{array}{c}.05 \\
{[-.25 ; .33]} \\
(.51) \\
\end{array}$ & $\begin{array}{c}-.04 \\
{[-.50 ; .36]} \\
(.53) \\
\end{array}$ & $\begin{array}{c}-.09 \\
{[-.45 ; .27]} \\
(.53) \\
\end{array}$ & $\begin{array}{c}-.02 \\
{[-.40 ; .37]} \\
(.55) \\
\end{array}$ & $\begin{array}{c}-.01 \\
{[-.39 ; .38]} \\
(.53) \\
\end{array}$ & $\begin{array}{c}-.10 \\
{[-.55 ; .33]} \\
(.54) \\
\end{array}$ & $\begin{array}{c}-.06 \\
{[-.50 ; .33]} \\
(.54) \\
\end{array}$ & $\begin{array}{c}-.04 \\
{[-.47 ; .38]} \\
(.54) \\
\end{array}$ & $\begin{array}{c}-.11 \\
{[-.53 ; .30]} \\
(.55) \\
\end{array}$ & $\begin{array}{c}-.08 \\
{[-.47 ; .26]} \\
(.54) \\
\end{array}$ \\
\hline & \multirow{3}{*}{$5 \%$} & Original & $\begin{array}{c}\text { Shape-par. } \\
{[5 \% ; 95 \%]} \\
\text { KS P-stat. }\end{array}$ & $\begin{array}{c}.15 \\
{[.01 ;-.22]} \\
(1.00) \\
\end{array}$ & $\begin{array}{c}-.05 \\
{[-.16 ; .08]} \\
(.55) \\
\end{array}$ & $\begin{array}{c}-.27 \\
{[-.38 ;-.12]} \\
(.24) \\
\end{array}$ & $\begin{array}{c}-.36 \\
{[-.46 ;-.25]} \\
(.90) \\
\end{array}$ & $\begin{array}{c}-.44 \\
{[-.60 ;-.29]} \\
(.03) \\
\end{array}$ & $\begin{array}{c}-.11 \\
{[-.23 ;-.02]} \\
(.11) \\
\end{array}$ & $\begin{array}{c}-.41 \\
{[-.58 ;-.25]} \\
(.55) \\
\end{array}$ & $\begin{array}{c}.04 \\
{[-.06 ;-.17]} \\
(.30) \\
\end{array}$ & $\begin{array}{c}-.02 \\
{[-.15 ;-.10]} \\
(.74) \\
\end{array}$ & $\begin{array}{c}-.10 \\
{[-.22 ;-.03]} \\
(.95) \\
\end{array}$ \\
\hline & & Bootstrap & $\begin{array}{l}\text { Shape-par. } \\
{[5 \% ; 95 \%]} \\
\text { KS P-stat. }\end{array}$ & $\begin{array}{c}.07 \\
{[-.27 ; .42]} \\
(.50) \\
\end{array}$ & $\begin{array}{c}-.08 \\
{[-.41 ; .24]} \\
(.53) \\
\end{array}$ & $\begin{array}{c}-.01 \\
{[-.31 ; .28]} \\
(.52) \\
\end{array}$ & $\begin{array}{c}-.08 \\
{[-.39 ; .21]} \\
(.51) \\
\end{array}$ & $\begin{array}{c}-.07 \\
{[-.39 ; .24]} \\
(.53) \\
\end{array}$ & $\begin{array}{c}-.13 \\
{[-.46 ; .16]} \\
(.52) \\
\end{array}$ & $\begin{array}{c}-.10 \\
{[-.44 ; .19]} \\
(.51) \\
\end{array}$ & $\begin{array}{c}-.11 \\
{[-.43 ; .19]} \\
(.53) \\
\end{array}$ & $\begin{array}{c}-.13 \\
{[-.45 ; .17]} \\
(.52) \\
\end{array}$ & $\begin{array}{c}-.10 \\
{[-.40 ; .17]} \\
(.54) \\
\end{array}$ \\
\hline & & Surrogate & $\begin{array}{c}\text { Shape-par. } \\
{[5 \% ; 95 \%]} \\
\text { KS P-stat. }\end{array}$ & $\begin{array}{c}.07 \\
{[-.15 ; .29]} \\
(.55) \\
\end{array}$ & $\begin{array}{c}-.08 \\
{[-.40 ; .22]} \\
(.54) \\
\end{array}$ & $\begin{array}{c}.00 \\
{[-.29 ; .26]} \\
(.52) \\
\end{array}$ & $\begin{array}{c}-.07 \\
{[-.36 ; .22]} \\
(.54) \\
\end{array}$ & $\begin{array}{c}-.06 \\
{[-.36 ; .24]} \\
(.51) \\
\end{array}$ & $\begin{array}{c}-.12 \\
{[-.45 ; .19]} \\
(.52) \\
\end{array}$ & $\begin{array}{c}-.08 \\
{[-.39 ; .21]} \\
(.53) \\
\end{array}$ & $\begin{array}{c}-.09 \\
{[-.41 ; .22]} \\
(.51) \\
\end{array}$ & $\begin{array}{c}-.14 \\
{[-.46 ; .16]} \\
(.52) \\
\end{array}$ & $\begin{array}{c}-.09 \\
{[-.37 ; .16]} \\
(.53) \\
\end{array}$ \\
\hline & \multirow{3}{*}{$10 \%$} & Original & $\begin{array}{c}\text { Shape-par. } \\
{[5 \% ; 95 \%]} \\
\text { KS P-stat. }\end{array}$ & $\begin{array}{c}-.23 \\
{[-.34 ;-.15]} \\
(.09) \\
\end{array}$ & $\begin{array}{c}-.24 \\
{[-.35 ;-.15]} \\
(.91) \\
\end{array}$ & $\begin{array}{c}.05 \\
{[-.08 ; .19]} \\
(.16) \\
\end{array}$ & $\begin{array}{c}-.18 \\
{[-.31 ;-.07]} \\
(.41) \\
\end{array}$ & $\begin{array}{c}.02 \\
{[-.09 ; .14]} \\
(.16) \\
\end{array}$ & $\begin{array}{c}-.29 \\
{[-.39 ;-.18]} \\
(.54)\end{array}$ & $\begin{array}{c}-.02 \\
{[-.12 ;-.09]} \\
(.67) \\
\end{array}$ & $\begin{array}{c}.01 \\
{[-.11 ; .12]} \\
(.07) \\
\end{array}$ & $\begin{array}{c}-.36 \\
{[-.47 ;-.23]} \\
(.03) \\
\end{array}$ & $\begin{array}{c}-.10 \\
{[-.21 ; .03]} \\
(.01) \\
\end{array}$ \\
\hline & & Bootstrap & $\begin{array}{c}\text { Shape-par. } \\
{[5 \% ; 95 \%]} \\
\text { KS P-stat. }\end{array}$ & $\begin{array}{c}-.02 \\
{[-.26 ; .21]} \\
(.46) \\
\end{array}$ & $\begin{array}{c}-.10 \\
{[-.35 ; .11]} \\
(.49) \\
\end{array}$ & $\begin{array}{c}-.14 \\
{[-.37 ; .08]} \\
(.48) \\
\end{array}$ & $\begin{array}{c}-.14 \\
{[-.36 ; .08]} \\
(.52) \\
\end{array}$ & $\begin{array}{c}-.13 \\
{[-.36 ; .08]} \\
(.51) \\
\end{array}$ & $\begin{array}{c}-.17 \\
{[-.40 ; .04]} \\
(.50) \\
\end{array}$ & $\begin{array}{c}-.12 \\
{[-.37 ; .09]} \\
(.51) \\
\end{array}$ & $\begin{array}{c}-.13 \\
{[-.37 ; .08]} \\
(.51) \\
\end{array}$ & $\begin{array}{c}-.21 \\
{[-.44 ; .00]} \\
(.52) \\
\end{array}$ & $\begin{array}{c}-.13 \\
{[-.34 ; .06]} \\
(.52) \\
\end{array}$ \\
\hline & & Surrogate & $\begin{array}{c}\text { Shape-par. } \\
{[5 \% ; 95 \%]} \\
\text { KS P-stat. }\end{array}$ & $\begin{array}{c}-.02 \\
{[-.20 ; .15]} \\
(.49) \\
\end{array}$ & $\begin{array}{c}-.10 \\
{[-.34 ; .11]} \\
(.50) \\
\end{array}$ & $\begin{array}{c}-.13 \\
{[-.34 ; .06]} \\
(.49) \\
\end{array}$ & $\begin{array}{c}-.14 \\
{[-.37 ; .07]} \\
(.50) \\
\end{array}$ & $\begin{array}{c}-.14 \\
{[-.36 ; .08]} \\
(.52)\end{array}$ & $\begin{array}{c}-.18 \\
{[-.41 ; .02]} \\
(.52) \\
\end{array}$ & $\begin{array}{c}-. .12 \\
{[-.35 ; .09]} \\
(.50) \\
\end{array}$ & $\begin{array}{c}-.14 \\
{[-.38 ; .09]} \\
(.53) \\
\end{array}$ & $\begin{array}{c}-.21 \\
{[-.42 ; .01]} \\
(.50) \\
\end{array}$ & $\begin{array}{c}-.13 \\
{[-.33 ; .05]} \\
(.51) \\
\end{array}$ \\
\hline
\end{tabular}

Source: Euronext, 30' resampled intraday CAC40 French stock index quotes from the period 01-01-1997/05-31-2009. Reported in this table are the empirical Shape-parameters for the various volatility measures. estimated by a Method of LQ-moments of a GPD with various quantiles of observations (thresholds for the peak definitions). The $90 \%$ confidence intervals are reported in brackets, whilst P-statistics of Goodness-of-Fit Kolmogorov-Smirnov tests (denoted KS P-stat.) are between parentheses. Computations by the authors. 
Table C.3-d: Estimates of Shape-parameters of Generalized Pareto Distributions

of Peaks-over-Threshold Daily Log-volatilities using a Method of LSd-moments

\begin{tabular}{|c|c|c|c|c|c|c|c|c|c|c|c|c|c|}
\hline LSd-moments & Frequencies & Series & Statistics & \begin{tabular}{|c} 
Realized \\
Volatility \\
\end{tabular} & $\begin{array}{l}\text { Bi-power } \\
\text { Variation } \\
\end{array}$ & $\begin{array}{l}\text { Jump } \\
\text { Comp. }\end{array}$ & Parkinson & Kunitomo & $\begin{array}{l}\text { Rogers- } \\
\text { Satchell } \\
\end{array}$ & $\begin{array}{c}\text { Garman- } \\
\text { Klass }\end{array}$ & $\begin{array}{l}\text { Yang- } \\
\text { Zhang }\end{array}$ & Simple & $\begin{array}{l}\text { Exponential } \\
\text { Moving Av. }\end{array}$ \\
\hline \multirow{9}{*}{ LSd-moments } & \multirow{3}{*}{$2.50 \%$} & Original & $\begin{array}{c}\text { Shape-par. } \\
{[5 \% ; 95 \%]} \\
\text { KS P-stat. }\end{array}$ & $\begin{array}{c}-.04 \\
{[-.10 ; .03]} \\
(.80) \\
\end{array}$ & $\begin{array}{c}.01 \\
{[-.06 ; .08]} \\
(.80) \\
\end{array}$ & $\begin{array}{c}-.15 \\
{[-.20 ;-.09]} \\
(.53) \\
\end{array}$ & $\begin{array}{c}-.18 \\
{[-.23 ;-.13]} \\
(.67) \\
\end{array}$ & $\begin{array}{c}-.13 \\
{[-.20 ;-.07]} \\
(.41) \\
\end{array}$ & $\begin{array}{c}-.24 \\
{[-.30 ;-.18]} \\
(.03) \\
\end{array}$ & $\begin{array}{c}-.31 \\
{[-.35 ;-.24]} \\
(.53) \\
\end{array}$ & $\begin{array}{c}-.13 \\
{[-.18 ;-.08]} \\
(.30) \\
\end{array}$ & $\begin{array}{c}-.06 \\
{[-.12 ;-.02]} \\
(.80) \\
\end{array}$ & $\begin{array}{c}-.03 \\
{[-.07 ; .04]} \\
(.15) \\
\end{array}$ \\
\hline & & Bootstrap & $\begin{array}{c}\text { Shape-par. } \\
\text { [5\%;95\%] } \\
\text { KS P-stat. }\end{array}$ & $\begin{array}{c}-.07 \\
{[-.18 ; .05]} \\
(.56) \\
\end{array}$ & $\begin{array}{c}-.04 \\
{[-.16 ; .08]} \\
(.60) \\
\end{array}$ & $\begin{array}{c}-.07 \\
{[-.19 ; .04]} \\
(.55) \\
\end{array}$ & $\begin{array}{c}-.06 \\
{[-.18 ; .05]} \\
(.58) \\
\end{array}$ & $\begin{array}{c}-.06 \\
{[-.18 ; .05]} \\
(.57) \\
\end{array}$ & $\begin{array}{c}-.07 \\
{[-.19 ; .05]} \\
(.58) \\
\end{array}$ & $\begin{array}{c}-.07 \\
{[-.18 ; .06]} \\
(.57) \\
\end{array}$ & $\begin{array}{c}-.04 \\
{[-.16 ; .08]} \\
(.61) \\
\end{array}$ & $\begin{array}{c}-.08 \\
{[-.18 ; .03]} \\
(.57) \\
\end{array}$ & $\begin{array}{c}-.07 \\
{[-.17 ; .03]} \\
(.59) \\
\end{array}$ \\
\hline & & Surrogate & $\begin{array}{c}\text { Shape-par. } \\
{[5 \% ; 95 \%]} \\
\text { KS P-stat. }\end{array}$ & $\begin{array}{c}-.06 \\
{[-.14 ; .00]} \\
(.62) \\
\end{array}$ & $\begin{array}{c}-.04 \\
{[-.16 ; .08]} \\
(.59) \\
\end{array}$ & $\begin{array}{c}-.07 \\
{[-.15 ; .02]} \\
(.60) \\
\end{array}$ & $\begin{array}{c}-.05 \\
{[-.16 ; .05]} \\
(.61) \\
\end{array}$ & $\begin{array}{c}-.05 \\
{[-.15 ; .05]} \\
(.58) \\
\end{array}$ & $\begin{array}{c}-.07 \\
{[-.18 ; .04]} \\
(.59) \\
\end{array}$ & $\begin{array}{c}-.06 \\
{[-.17 ; .06]} \\
(.59) \\
\end{array}$ & $\begin{array}{c}-.03 \\
{[-.16 ; .09]} \\
(.58) \\
\end{array}$ & $\begin{array}{c}-.08 \\
{[-.18 ; .02]} \\
(.59) \\
\end{array}$ & $\begin{array}{c}-.06 \\
{[-.15 ; .03]} \\
(.57) \\
\end{array}$ \\
\hline & \multirow{3}{*}{$5 \%$} & Original & $\begin{array}{c}\text { Shape-par. } \\
{[5 \% ; 95 \%]} \\
\text { KS P-stat. }\end{array}$ & $\begin{array}{c}.01 \\
{[-.03 ; .06]} \\
(.99) \\
\end{array}$ & $\begin{array}{c}-.02 \\
{[-.06 ; .04]} \\
(.37) \\
\end{array}$ & $\begin{array}{c}-.11 \\
{[-.16 ;-.03]} \\
(.64) \\
\end{array}$ & $\begin{array}{c}-.15 \\
{[-.22 ;-.11]} \\
(.99) \\
\end{array}$ & $\begin{array}{c}-.09 \\
{[-.13 ;-.02]} \\
(.24) \\
\end{array}$ & $\begin{array}{c}-.12 \\
{[-.19 ;-.06]} \\
(.24) \\
\end{array}$ & $\begin{array}{c}-.15 \\
{[-.19 ;-.10]} \\
(.02) \\
\end{array}$ & $\begin{array}{c}-.05 \\
{[-.10 ; .00]} \\
(.46) \\
\end{array}$ & $\begin{array}{c}-.09 \\
{[-.14 ;-.02]} \\
(.74) \\
\end{array}$ & $\begin{array}{c}-.08 \\
{[-.15 ;-.02]} \\
(.55) \\
\end{array}$ \\
\hline & & Bootstrap & $\begin{array}{c}\text { Shape-par. } \\
\text { [5\%;95\%] } \\
\text { KS P-stat. }\end{array}$ & $\begin{array}{c}.01 \\
{[-.08 ; .10]} \\
(.57) \\
\end{array}$ & $\begin{array}{c}-.05 \\
{[-.14 ; .04]} \\
(.56) \\
\end{array}$ & $\begin{array}{c}-.04 \\
{[-.12 ; .05]} \\
(.55) \\
\end{array}$ & $\begin{array}{c}-.06 \\
{[-.13 ; .02]} \\
(.56) \\
\end{array}$ & $\begin{array}{c}-.06 \\
{[-.13 ; .02]} \\
(.54) \\
\end{array}$ & $\begin{array}{c}-.07 \\
{[-.15 ; .01]} \\
(.52) \\
\end{array}$ & $\begin{array}{c}-.06 \\
{[-.15 ; .02]} \\
(.54) \\
\end{array}$ & $\begin{array}{c}-.05 \\
{[-.15 ; .03]} \\
(.54) \\
\end{array}$ & $\begin{array}{c}-.08 \\
{[-.17 ;--.01]} \\
(.53) \\
\end{array}$ & $\begin{array}{c}-.06 \\
{[-.14 ; .01]} \\
(.56) \\
\end{array}$ \\
\hline & & Surrogate & $\begin{array}{c}\text { Shape-par. } \\
{[5 \% ; 95 \%]} \\
\text { KS P-stat. }\end{array}$ & $\begin{array}{c}.02 \\
{[-.04 ; .07]} \\
(.60) \\
\end{array}$ & $\begin{array}{c}-.05 \\
{[-.14 ; .04]} \\
(.55) \\
\end{array}$ & $\begin{array}{c}-.03 \\
{[-.10 ; .03]} \\
(.57) \\
\end{array}$ & $\begin{array}{c}-.06 \\
{[-.13 ; .02]} \\
(.56) \\
\end{array}$ & $\begin{array}{c}-.05 \\
{[-.13 ; .03]} \\
(.56) \\
\end{array}$ & $\begin{array}{c}-.07 \\
{[-.16 ; .02]} \\
(.54) \\
\end{array}$ & $\begin{array}{c}-.05 \\
{[-.14 ; .02]} \\
(.56) \\
\end{array}$ & $\begin{array}{c}-.05 \\
{[-.14 ; .04]} \\
(.56) \\
\end{array}$ & $\begin{array}{c}-.08 \\
{[-.16 ;-.01]} \\
(.52) \\
\end{array}$ & $\begin{array}{c}-.06 \\
{[-.14 ; .01]} \\
(.55) \\
\end{array}$ \\
\hline & \multirow{3}{*}{$10 \%$} & Original & $\begin{array}{c}\text { Shape-par. } \\
{[5 \% ; 95 \%]} \\
\text { KS P-stat. }\end{array}$ & $\begin{array}{c}-.13 \\
{[-.19 ;-.05]} \\
(.19) \\
\end{array}$ & $\begin{array}{c}-.07 \\
{[-.14 ;-.02]} \\
(.80) \\
\end{array}$ & $\begin{array}{c}-.02 \\
{[-.06 ; .03]} \\
(.11) \\
\end{array}$ & $\begin{array}{c}-.10 \\
{[-.16 ;-.06]} \\
(.04) \\
\end{array}$ & $\begin{array}{c}-.10 \\
{[-.15 ;-.04]} \\
(.16) \\
\end{array}$ & $\begin{array}{c}-.12 \\
{[-.17 ;-.05]} \\
(.06) \\
\end{array}$ & $\begin{array}{c}-.08 \\
{[-.14 ;-.01]} \\
(.54) \\
\end{array}$ & $\begin{array}{c}-.07 \\
{[-.11 ;-.01]} \\
(.01) \\
\end{array}$ & $\begin{array}{c}-.14 \\
{[-.19 ;-.08]} \\
(.03) \\
\end{array}$ & $\begin{array}{c}-.13 \\
{[-.18 ;-.08]} \\
(.67) \\
\end{array}$ \\
\hline & & Bootstrap & $\begin{array}{c}\text { Shape-par. } \\
{[5 \% ; 95 \%]} \\
\text { KS P-stat. }\end{array}$ & $\begin{array}{c}-.03 \\
{[-.09 ; .04]} \\
(.50) \\
\end{array}$ & $\begin{array}{c}-.06 \\
{[-.12 ; .00]} \\
(.51) \\
\end{array}$ & $\begin{array}{c}-.08 \\
{[-.14 ;-.01]} \\
(.43) \\
\end{array}$ & $\begin{array}{c}-.07 \\
{[-.13 ;-.01]} \\
(.46) \\
\end{array}$ & $\begin{array}{c}-.07 \\
{[-.13 ;-.01]} \\
(.48) \\
\end{array}$ & $\begin{array}{c}-.09 \\
{[-.15 ;-.03]} \\
\quad(.39) \\
\end{array}$ & $\begin{array}{c}-.07 \\
{[-.13 ;-.01]} \\
(.46) \\
\end{array}$ & $\begin{array}{c}-.07 \\
{[-.13 ;-.01]} \\
(.46) \\
\end{array}$ & $\begin{array}{c}-.11 \\
{[-.17 ;--.05]} \\
(.35) \\
\end{array}$ & $\begin{array}{c}-.08 \\
{[-.14 ;-.03]} \\
(.45) \\
\end{array}$ \\
\hline & & Surrogate & $\begin{array}{c}\text { Shape-par. } \\
{[5 \% ; 95 \%]} \\
\text { KS P-stat. }\end{array}$ & $\begin{array}{c}-.03 \\
{[-.07 ; ; 01]} \\
(.56) \\
\end{array}$ & $\begin{array}{c}-.06 \\
{[-.12 ; .00]} \\
(.48) \\
\end{array}$ & $\begin{array}{c}-.08 \\
{[-.13 ;-.03]} \\
\quad(.44) \\
\end{array}$ & $\begin{array}{c}-.07 \\
{[-.13 ;-.02]} \\
(.47) \\
\end{array}$ & $\begin{array}{c}-.07 \\
{[-.12 ;-.01]} \\
(.49) \\
\end{array}$ & $\begin{array}{c}-.09 \\
{[-.15 ;-.03]} \\
(.38) \\
\end{array}$ & $\begin{array}{c}-.07 \\
{[-.13 ;-.02]} \\
\quad(.44) \\
\end{array}$ & $\begin{array}{c}-.07 \\
{[-.13 ;-.01]} \\
(.47) \\
\end{array}$ & $\begin{array}{c}-.11 \\
{[-.16 ;-.06]} \\
(.36) \\
\end{array}$ & $\begin{array}{c}-.08 \\
{[-.13 ;-.02]} \\
(.48) \\
\end{array}$ \\
\hline
\end{tabular}

Source: Euronext, 30' resampled intraday CAC40 French stock index quotes from the period 01-01-1997/05-31-2009. Reported in this table are the empirical Shape-parameters for the various volatility measures. estimated by a Method of LSd-moments of a GPD with various quantiles of observations (thresholds for the peak definitions). The 90\% confidence intervals are reported in brackets, whilst P-statistics of Goodness-of-Fit Kolmogorov-Smirnov tests (denoted KS P-stat.) are between parentheses. Computations by the authors. 
Table C-4: Comparison of Estimates of Shape-parameters

of Generalized Extreme Value Distributions of Weekly Maxima

of Daily Log-volatilities using Maximum Likelihood and Various Bootstrap Methods

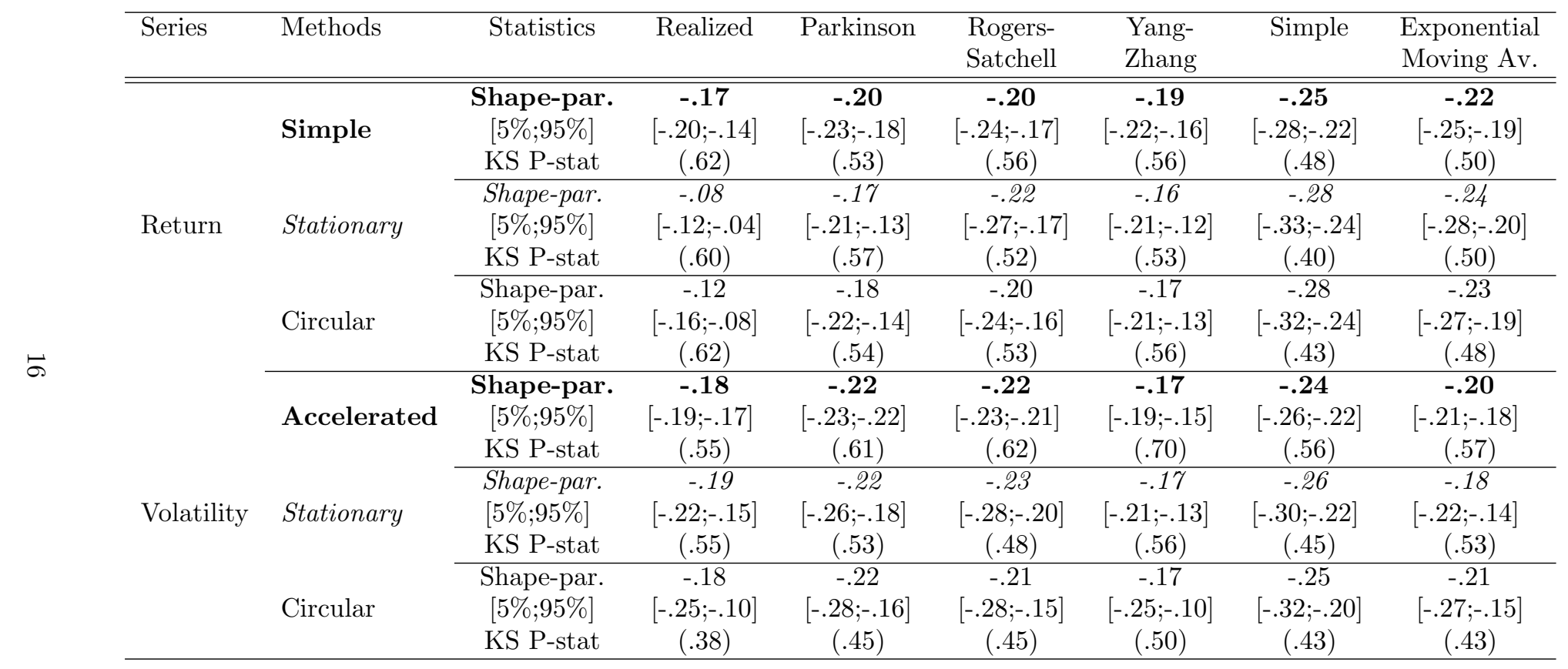

Source: Euronext, 30' resampled intraday CAC40 French stock index quotes from the period 01-01-1997/05-31-2009. The Shape-parameters for the various volatility measures are estimated by the Method of Maximum Likelihood of a GEV density with a weekly frequency (the block maxima length) on 10,000 series obtained with bootstrap methods (simple: Efron and Tibshirani, 1994; stationary: Politis and Romano, 1994; circular: Politis and Romano, 1994, and Politis and White, 2004; accelerated: Efron and Tibshirani, 1994, and Gilli and Këllezi, 2006) on series of returns or on series of volatilities. The $90 \%$ confidence intervals of Shape-parameters are reported in brackets, whilst P-statistics of Goodness-of-Fit Kolmogorov-Smirnov tests (denoted KS P-stat.) are between parentheses. Computations by the authors. 
Table C-5: Comparison of Estimates of Shape-parameters

of Generalized Extreme Value Distributions of Weekly Maxima of Daily Log-volatilities

using Maximum Likelihood, L-moments, T(1,1)-moments and Different Surrogate Methods

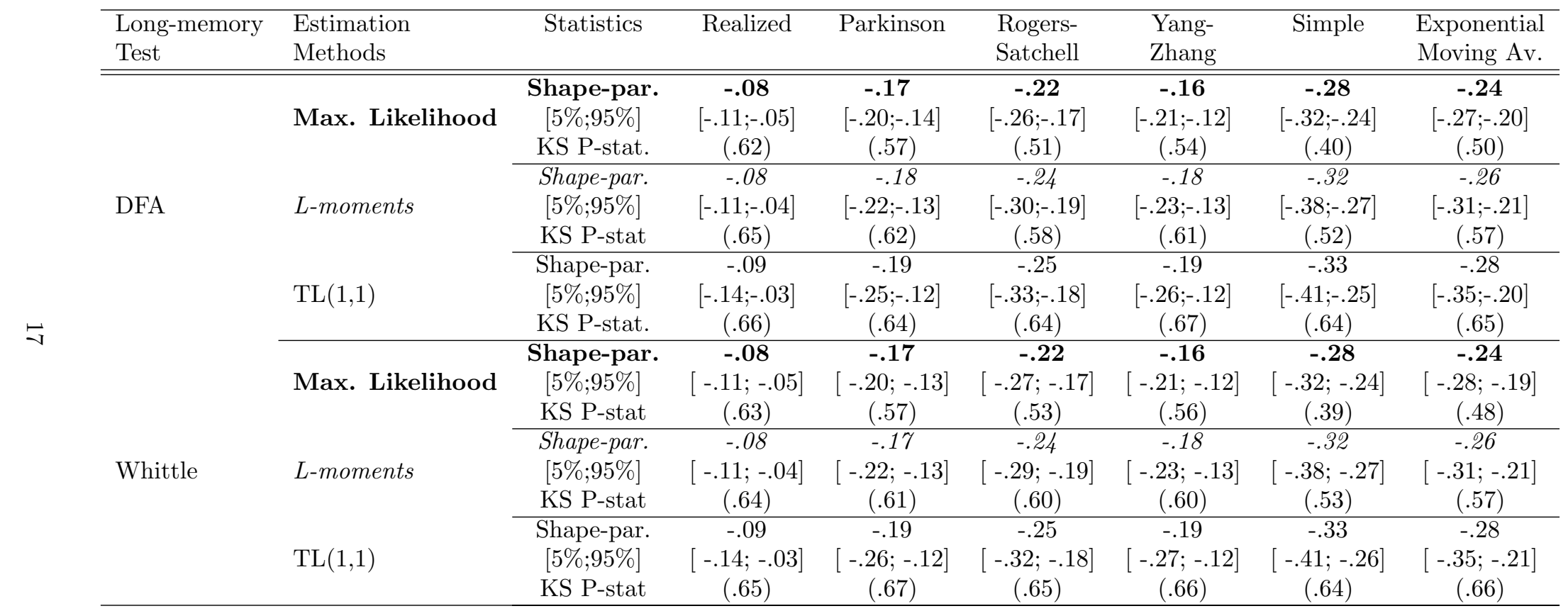

Source: Euronext, 30' resampled intraday CAC40 French stock index quotes from the period 01-01-1997/05-31-2009. The Shape-parameters for the various volatility measures are estimated by the Methods of Maximum Likelihood, L-moments and T(1,1)-moments of a GEV density with a weekly frequency (the block maxima length) on 10,000 series obtained with surrogate methods (DFA: Kantelhardt et al., 2001; Whittle: Künsch, 1986) on series of volatilities. The 90\% confidence intervals of Shape-parameters are reported in brackets, whilst P-statistics of Goodness-of-Fit Kolmogorov-Smirnov tests (denoted KS P-stat.) are between parentheses. Computations by the authors. 
Table C-6: Comparison of Estimates of Shape-parameters

of Generalized Extreme Value Distributions of Weekly Maxima

of Daily Log-volatilities using Maximum Likelihood and Various Frequencies

\begin{tabular}{|c|c|c|c|c|c|c|c|c|}
\hline Series & Frequencies & Statistics & Realized & Parkinson & $\begin{array}{l}\text { Rogers- } \\
\text { Satchell }\end{array}$ & $\begin{array}{l}\text { Yang- } \\
\text { Zhang }\end{array}$ & Simple & $\begin{array}{l}\text { Exponential } \\
\text { Moving Av. }\end{array}$ \\
\hline \multirow{3}{*}{ Volatility } & 30 minutes & $\begin{array}{c}\text { Shape-par. } \\
{[5 \% ; 95 \%]} \\
\text { KS P-stat }\end{array}$ & $\begin{array}{c}-.18 \\
{[-.22 ;-.14]} \\
(.56)\end{array}$ & $\begin{array}{c}-.22 \\
{[-.26 ;-.18]} \\
(.53)\end{array}$ & $\begin{array}{c}-.23 \\
{[-.27 ;-.19]} \\
(.49)\end{array}$ & $\begin{array}{c}-.17 \\
{[-.21 ;-.13]} \\
(.56)\end{array}$ & $\begin{array}{c}-.17 \\
{[-.21 ;-.13]} \\
(.54)\end{array}$ & $\begin{array}{c}-.25 \\
{[-.29 ;-.21]} \\
(.45)\end{array}$ \\
\hline & 60 minutes & $\begin{array}{c}\text { Shape-par. } \\
{[5 \% ; 95 \%]} \\
\text { KS P-stat }\end{array}$ & $\begin{array}{c}-.19 \\
{[-.22 ;-.16]} \\
(.57)\end{array}$ & $\begin{array}{c}-.22 \\
{[-.22 ;-.21]} \\
(.61)\end{array}$ & $\begin{array}{c}-.23 \\
{[-.25 ;-.20]} \\
(.61)\end{array}$ & $\begin{array}{c}-.17 \\
{[-.19 ;-.15]} \\
(.72)\end{array}$ & $\begin{array}{c}-.17 \\
{[-.21 ;-.18]} \\
(.54)\end{array}$ & $\begin{array}{c}-.25 \\
{[-.29 ;-.20]} \\
(.53)\end{array}$ \\
\hline & 120 minutes & $\begin{array}{c}\text { Shape-par. } \\
{[5 \% ; 95 \%]} \\
\text { KS P-stat }\end{array}$ & $\begin{array}{c}-.18 \\
{[-.19 ;-.17]} \\
(.61)\end{array}$ & $\begin{array}{c}-.22 \\
{[-.22 ;-.21]} \\
(.60)\end{array}$ & $\begin{array}{c}-.23 \\
{[-.25 ;-.19]} \\
(.61)\end{array}$ & $\begin{array}{c}-.17 \\
{[-.19 ;-.15]} \\
(.70)\end{array}$ & $\begin{array}{c}-.17 \\
{[-.21 ;-.18]} \\
(.57)\end{array}$ & $\begin{array}{c}-.25 \\
{[-.29 ;-.22]} \\
(.54)\end{array}$ \\
\hline
\end{tabular}

Source: Euronext, 30', 60' and 120' resampled intraday CAC40 French stock index quotes from the period 01-01-1997/05-31-2009. The Shapeparameters for the various volatility measures are estimated by the Method of Maximum Likelihood of a GEV density with a weekly frequency (the block maxima length) on 10,000 series obtained with the stationary bootstrap method (Politis and Romano, 1994) on series of volatilities. The $90 \%$ confidence intervals of Shape- parameters are reported in brackets, whilst P-statistics of Goodness-of-Fit Kolmogorov-Smirnov tests (denoted KS P-stat.) are between parentheses. Computations by the authors. 
Table C-7: Comparison of Estimates of Shape-parameters

of Generalized Extreme Value Distributions of Weekly Maxima

of Daily Log-volatilities using Maximum Likelihood for Various Sample Periods

\begin{tabular}{|c|c|c|c|c|c|c|c|c|}
\hline Series & Periods & Statistics & Realized & Parkinson & $\begin{array}{l}\text { Rogers- } \\
\text { Satchell }\end{array}$ & $\begin{array}{l}\text { Yang- } \\
\text { Zhang }\end{array}$ & Simple & $\begin{array}{l}\text { Exponential } \\
\text { Moving Av. }\end{array}$ \\
\hline & 1997-2009 & $\begin{array}{c}\text { Shape-par. } \\
{[5 \% ; 95 \%]} \\
\text { KS P-stat }\end{array}$ & $\begin{array}{c}-.18 \\
{[-.22 ;-.14]} \\
(.56)\end{array}$ & $\begin{array}{c}-.21 \\
{[-.26 ;-.18]} \\
(.53)\end{array}$ & $\begin{array}{c}-.23 \\
{[-.27 ;-.19]} \\
(.49)\end{array}$ & $\begin{array}{c}-.17 \\
{[-.21 ;-.13]} \\
(.56)\end{array}$ & $\begin{array}{c}-.17 \\
{[-.21 ;-.13]} \\
(.54)\end{array}$ & $\begin{array}{c}-.25 \\
{[-.29 ;-.21]} \\
(.45)\end{array}$ \\
\hline Volatility & 1997-2000 & $\begin{array}{c}\text { Shape-par. } \\
{[5 \% ; 95 \%]} \\
\text { KS P-stat }\end{array}$ & $\begin{array}{c}-.18 \\
{[-.19 ;-.17]} \\
(.58) \\
\end{array}$ & $\begin{array}{c}-.22 \\
{[-.22 ;-.21]} \\
(.61) \\
\end{array}$ & $\begin{array}{c}-.21 \\
{[-.22 ;-.20]} \\
(.59) \\
\end{array}$ & $\begin{array}{c}-.17 \\
{[-.19 ;-.15]} \\
(.69)\end{array}$ & $\begin{array}{c}-.19 \\
{[-.21 ;-.18]} \\
(.55) \\
\end{array}$ & $\begin{array}{c}-.23 \\
{[-.25 ;-.21]} \\
(.54)\end{array}$ \\
\hline & $1997-2007$ & $\begin{array}{c}\text { Shape-par. } \\
{[5 \% ; 95 \%]} \\
\text { KS P-stat }\end{array}$ & $\begin{array}{c}-.18 \\
{[-.19 ;-.17]} \\
(.58)\end{array}$ & $\begin{array}{c}-.22 \\
{[-.22 ;-.21]} \\
(.64)\end{array}$ & $\begin{array}{c}-.21 \\
{[-.22 ;-.20]} \\
(.60)\end{array}$ & $\begin{array}{c}-.17 \\
{[-.19 ;-.15]} \\
(.70)\end{array}$ & $\begin{array}{c}-.19 \\
{[-.21 ;-.18]} \\
(.56)\end{array}$ & $\begin{array}{c}-.23 \\
{[-.25 ;-.21]} \\
(.53)\end{array}$ \\
\hline
\end{tabular}

Source: Euronext, 30' resampled intraday CAC40 French stock index quotes from the periods 01-01-1997/05-31-2009, 01-01-1997/01-01-2000 and 01-01-1997/01-01-2007. The Shape-parameters for the various volatility measures are estimated by the Method of Maximum Likelihood of a GEV density with a weekly frequency (the block maxima length) on 10,000 series obtained with the stationary bootstrap method (Politis and Romano, 1994) on series of volatilities. The $90 \%$ confidence intervals of Shape-parameters are reported in brackets, whilst P-statistics of Goodness-of-Fit Kolmogorov-Smirnov tests (denoted KS P-stat.) are between parentheses. Computations by the authors. 
Table C-8: Comparison of Estimates of Shape-parameters of Generalized Extreme Value Distributions of Weekly Maxima of Daily Log-volatilities using Maximum Likelihood for Various Sample Periods conditioned to VIX and IMS Levels

\begin{tabular}{|c|c|c|c|c|c|c|c|c|}
\hline Criteria & Level & Statistics & Realized & Parkinson & $\begin{array}{l}\text { Rogers- } \\
\text { Satchell }\end{array}$ & $\begin{array}{l}\text { Yang- } \\
\text { Zhang }\end{array}$ & Simple & $\begin{array}{l}\text { Exponential } \\
\text { Moving Av. }\end{array}$ \\
\hline \multirow{9}{*}{ VIX } & \multirow{3}{*}{ Low } & Shape-par. & -.08 &.- .17 & -.12 & -.09 & -.23 & -.35 \\
\hline & & {$[5 \% ; 95 \%]$} & {$[-.13 ;-.02]$} & {$[-.21 ;-.11]$} & {$[-.16 ;-.06]$} & {$[-.15 ;-.02]$} & {$[-.27 ;-.17]$} & {$[-.37 ;-.31]$} \\
\hline & & KS P-stat & .11 & .73 & .45 & .54 & .30 & .90 \\
\hline & \multirow{3}{*}{ Median } & Shape-par. & -.12 & -.23 & -.18 & -.07 & -.24 & -.27 \\
\hline & & {$[5 \% ; 95 \%]$} & {$[-.16 ;-.06]$} & {$[-.26 ;-.19]$} & {$[-.22 ;-.14]$} & {$[-.12 ;-.01]$} & {$[-.26 ;-.21]$} & {$[-.28 ;-.24]$} \\
\hline & & KS P-stat & .91 & .31 & .47 & .80 & .16 & .19 \\
\hline & \multirow{3}{*}{ High } & Shape-par. & -.23 & -.23 & -.25 & -.21 & -.17 & -.25 \\
\hline & & {$[5 \% ; 95 \%]$} & {$[-.28 ;-.16]$} & {$[-.27 ;-.15]$} & {$[-.30 ;-.17]$} & {$[-.27 ;-.13]$} & {$[-.23 ;-.09]$} & {$[-.3 ;-.18]$} \\
\hline & & KS P-stat & .18 & .95 & .90 & .73 & .37 & .73 \\
\hline \multirow{9}{*}{ IMS } & \multirow{3}{*}{ Low } & Shape-par. & -.17 & -.25 & -.21 & -.16 & -.28 & -.34 \\
\hline & & {$[5 \% ; 95 \%]$} & {$[-.23 ;-.08]$} & {$[-.31 ;-.17]$} & {$[-.26 ;-.12]$} & {$[-.23 ;-.07]$} & {$[-.32 ;-.20]$} & {$[-.36 ;-.28]$} \\
\hline & & KS P-stat & .45 & .54 & .64 & .90 & .30 & .95 \\
\hline & \multirow{3}{*}{ Median } & Shape-par. & -.17 & -.22 & -.21 & -.12 & -.15 & -.19 \\
\hline & & {$[5 \% ; 95 \%]$} & {$[-.21 ;-.11]$} & {$[-.27 ;-.17]$} & {$[-.25 ;-.15]$} & {$[-.17 ;-.05]$} & {$[-.19 ;-.09]$} & {$[-.22 ;-.14]$} \\
\hline & & KS P-stat & .67 & .67 & .95 & .35 & .26 & .02 \\
\hline & \multirow{3}{*}{ High } & Shape-par. & -.10 & -.18 & -.21 & -.12 & -.03 & -.12 \\
\hline & & {$[5 \% ; 95 \%]$} & {$[-.18 ; .00]$} & {$[-.24 ;-.10]$} & {$[-.27 ;-.13]$} & {$[-.19 ;-.02]$} & {$[-.12 ; .07]$} & {$[-.19 ;-.02]$} \\
\hline & & KS P-stat & .64 & .73 & .19 & .90 & .98 & .95 \\
\hline
\end{tabular}

Source: Euronext, 30' resampled intraday CAC40 French stock index quotes from the periods 01-01-1997/05-31-2009. The Shape-parameters for the various volatility measures are estimated by the Method of Maximum Likelihood of a GEV density with a weekly frequency (the block maxima length) on 10,000 series obtained with the stationary bootstrap method (Politis and Romano, 1994) on series of volatilities obtained on dates defined by the level of the VIX (CBOT Implied Volatility Index) or the level of the Index of Market Shocks (IMS - see Maillet and Michel, 2003 and 2005). Low level corresponds to values below the overall 25\% quantile; Median level to values between the $25 \%$ and $75 \%$ quantiles; High level to values above the $75 \%$ quantile, for both VIX or IMS. The $90 \%$ confidence intervals of Shape- parameters are reported in brackets, whilst P-statistics of Goodness-of-Fit Kolmogorov-Smirnov tests (denoted KS P-stat.) are between parentheses. Computations by the authors. 


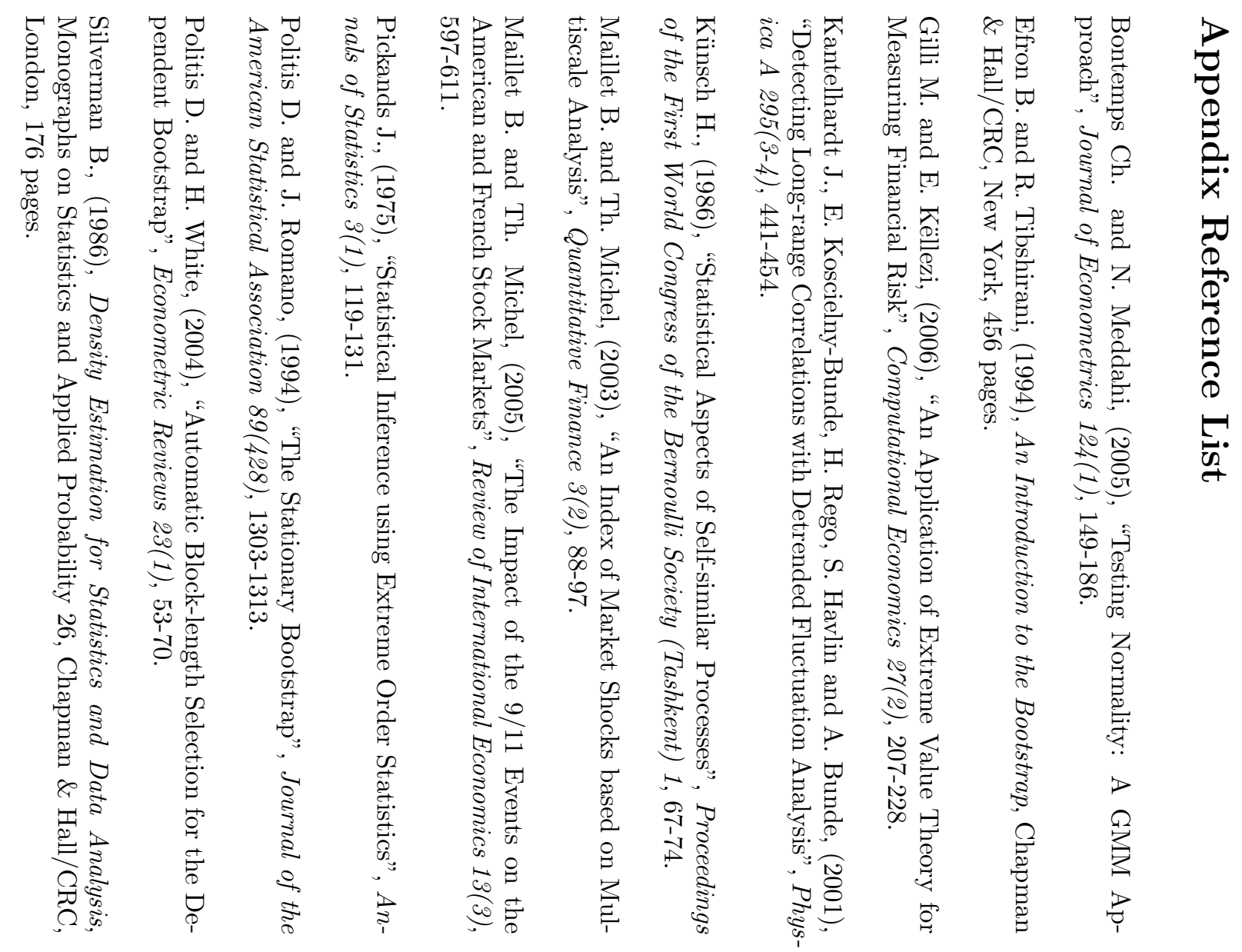

